Florida International University

FIU Digital Commons

$6-27-2019$

\title{
Nucleoside Analogues for Positron Emission Tomography Imaging and to Study Radiation Mediated Generation of Radicals from Azides
}

Maria E. de Cabrera

Florida International University, mdeca009@fiu.edu

Follow this and additional works at: https://digitalcommons.fiu.edu/etd

Part of the Nucleic Acids, Nucleotides, and Nucleosides Commons

\section{Recommended Citation}

de Cabrera, Maria E., "Nucleoside Analogues for Positron Emission Tomography Imaging and to Study Radiation Mediated Generation of Radicals from Azides" (2019). FIU Electronic Theses and Dissertations. 4229.

https://digitalcommons.fiu.edu/etd/4229

This work is brought to you for free and open access by the University Graduate School at FIU Digital Commons. It has been accepted for inclusion in FIU Electronic Theses and Dissertations by an authorized administrator of FIU Digital Commons. For more information, please contact dcc@fiu.edu. 


\title{
FLORIDA INTERNATIONAL UNIVERSITY
}

Miami, Florida

\section{NUCLEOSIDE ANALOGUES FOR POSITRON EMISSION TOMOGRAPHY IMAGING AND TO STUDY RADIATION MEDIATED GENERATION OF RADICALS FROM AZIDES}

\author{
A dissertation submitted in partial fulfillment of the \\ requirements for the degree of \\ DOCTOR IN PHILOSOPHY \\ in \\ CHEMISTRY
}

by

Maria de Cabrera

2019 
To: Dean Michael R. Heithaus

College of Arts, Sciences and Education

This dissertation, written by Maria de Cabrera, and entitled Nucleoside Analogues for Positron Emission Tomography Imaging and to Study Radiation Mediated Generation of Radicals from Azides, having been approved in respect to style and intellectual content, is referred to you for judgment.

We have read this dissertation and recommend that it be approved.

Raphael Raptis

Kathleen Rein

Jose Almirall

M. Alejandro Barbieri

Stanislaw F. Wnuk, Major Professor

Date of Defense: June 27, 2019

The dissertation of Maria de Cabrera is approved.

Dean Michael R. Heithaus
College of Arts, Sciences and Education

College of Arts, Sciences and Education

Andrés G. Gil

Vice President of Research and Economic Development and Dean of the University Graduate School

Florida International University, 2019 
(C) Copyright 2019 by Maria de Cabrera

All rights reserved. 


\section{DEDICATION}

I would like to dedicate this dissertation to all the people in my life that have, in one way or another, helped me to grow as person over these last years. 


\section{ACKNOWLEDGMENTS}

I would like to thank my advisor, Dr. Stanislaw F. Wnuk, for his support and guidance over these last four years. I would also like to thank my committee members: Dr. Raphael Raptis, Dr. Kathleen Rein, Dr. Jose Almirall, and Dr. Alejandro Barbieri for their support and advice throughout my graduate studies. I am very thankful for your insight and novel perspectives about my presentations and projects. I would like to extend my gratitude towards everyone in the Department of Chemistry and Biochemistry at Florida International University for all their help and support. I would also like to thank all my lab mates, past and present, for all their help day to day.

I would like to thank all of my collaborators, without whom this dissertation would not be possible. I would like to thank Dr. Anthony McGoron, from FIU's Department of Biomedical Engineering, and Dr. Alejandro Amor-Coarasa, from Weill Cornell Medical College, for their ${ }^{68} \mathrm{Ga}$ radiolabeling and in vivo rat studies of $4-\mathrm{N}$-alkylgemcitabine NOTA conjugates. Also, I would like to thank Dr. Caius Radu, from University of California Los Angeles, for sharing with us his unpublished biological studies of clofarabine ProTides, which were the basis for our design of clofarabine 5'-diphosphate prodrugs. I would like to thank Dr. Michael Sevilla and Dr. Amitava Adhikary, from Oakland University, for sharing with us their unpublished EPR results of the azido-modified nucleosides prepared in my dissertation. I would like to thank Dr. Adhikary for all his time and effort in helping me understand EPR results and properly explain them in this dissertation. Finally, I would like to thank Dr. Rupak Pathak from University of Arkansas for Medical Sciences for the biological evaluation of the radiosensitizing potential of azido-modified nucleosides. 
I want to express my sincere gratitude towards my family and friends. First and foremost, I would like to thank to my parents, Silvia and Carlos, who have had nearly godly patience with me throughout all my studies and have always supported me in every way they could. I would also like to thank by little brother, Carlos Jr., for always listening to me; you know our conversations are therapeutic for me. I would like to thank my boyfriend, Santiago, for always being there to support me over these last years; you are the kindest person I know. Finally, I would like to thank all my friends in the program and outside for all their help day to day; especially Vanessa for always listening to me and helping me in any way she could. 


\section{ABSTRACT OF THE DISSERTATION \\ NUCLEOSIDE ANALOGUES FOR POSITRON EMISSION TOMOGRAPHY \\ IMAGING AND TO STUDY RADIATION MEDIATED GENERATION OF \\ RADICALS FROM AZIDES}

by

Maria de Cabrera

Florida International University, 2019

Miami, Florida

Professor Stanislaw F. Wnuk, Major Professor

Gemcitabine is a potent anticancer cytidine analogue used to treat solid tumors. Its efficacy is diminished by rapid deamination to a toxic uridine derivative by cytidine deaminase. To overcome this limitation and add radioactive nuclei $\left({ }^{18} \mathrm{~F}\right.$ or $\left.{ }^{68} \mathrm{Ga}\right)$ for PET imaging, I synthesized two 4- $N$-alkylgemcitabine analogues i) bearing $\beta$-keto tosylate moiety for subsequent ${ }^{18} \mathrm{~F}$-fluorination and ii) having SCN-Bn-NOTA chelator to complex ${ }^{68} \mathrm{Ga}$. The first was synthesized by replacement of tosylamide in 4-N-tosylgemcitabine with 1-amino-10-undecene, followed by elaboration of terminal alkene through dihydroxylation, regioselective tosylation and oxidation. Subsequent fluorination using KF in presence of 18 -Crown- 6 at $75^{\circ} \mathrm{C}$ for $1 \mathrm{hr}$ gave $4-N$-alkylgemcitabine fluoromethyl ketone. The second was synthesized by analogous replacement of tosylamide with $N$-Boc1,3-propanediamine, followed by deprotection with TFA. The reactive terminal amine was condensed with SCN-Bn-NOTA, giving 4- $N$-alkylgemcitabine-SCN-Bn-NOTA ligand, which efficiently complexed Ga or ${ }^{68} \mathrm{Ga}$ for in vivo PET studies in rats. 
Clofarabine is a highly effective chemotherapeutic adenosine analogue used for treatment of acute lymphoblastic leukemia. Clofarabine undergoes rate limiting phosphorylation from its 5'-monophosphate to 5'-diphosphate by purine monophosphate kinase, and possible dephosphorylation of its respective 5'-monophosphate by 5'nucleotidases. Synthesis of clofarabine diphosphate prodrugs, and potentially their ${ }^{18} \mathrm{~F}-$ radiolabeled analogues, were undertaken to overcome these limitations. Successful synthesis of model adenosine diphosphate prodrug, by coupling adenosine monophosphate with bis(benzoyloxybenzyl) phosphoramidite in presence of 5-phenyl-1- $H$-tetrazole activator was achieved.

The aminyl radical generated from azide moiety in 3'-azido-3'-deoxythymidine (3'AZT) or 5-azidomethyl-2'-deoxyuridine (AmdU), upon addition of radiation-produced electrons, is thought to be the source of their radiosensitizing effects. Herein, I report synthesis of azido-modified purine and pyrimidine analogues for EPR study of formation of reactive aminyl radical in guanine, adenine and cytidine bases. The EPR studies of electron addition to 2-azidoguanosine (i.e. 2-azidoinsoine), protected 4-azidocytidine and 4-tetrazolocytidine analogues clearly establish that the position of the azide in base moiety dictates reactivity. The azide directly attached to nucleobases at ortho/para position to ring nitrogens produce stable $\mathrm{RN}_{3} \bullet^{-}$that does not rapidly convert to aminyl radical, except in the excited state. Hence, these did not display much radiosensitizing effects in in vivo biological studies in MDA-MB-231, MCF7 and U87 cell lines. 


\section{TABLE OF CONTENT}

CHAPTER

PAGE

1 INTRODUCTION 1

1.1 Anti-cancer properties of nucleoside analogues.............................................. 1

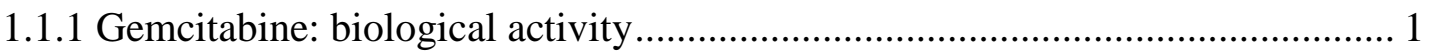

1.1.2 Clofarabine: biological activity ............................................................. 3

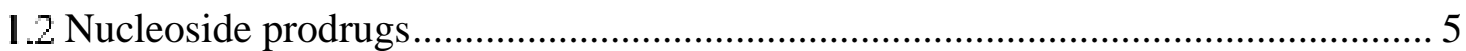

1.3 Positron Emission Tomography (PET) imaging ............................................... 9

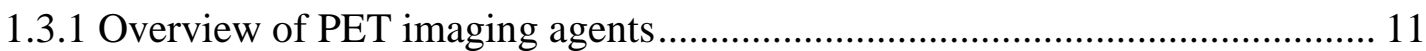

1.3.1.1 Nucleoside- and carbohydrate-based ${ }^{18}$ F-PET imaging agents ................ 11

1.3.1.2 ${ }^{68} \mathrm{Ga}$ based radiopharmaceuticals ........................................................ 13

1.4 Radical induced DNA damage in radiotherapy................................................ 14

1.4.1 Radical formation on the guanine base caused by DNA oxidation .................. 18

1.4.2 Studies on the formation of guanyl and adenyl aminyl radical ...................... 19

1.4.3 Radical formation in DNA oxidation of pyrimidine bases ............................ 22

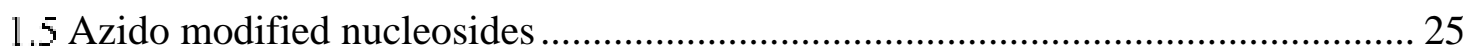

1.5.1 Synthesis and properties of azido modified nucleosides ............................... 26

1.5.1.1 Synthesis of azido modified purine nucleosides ................................... 26

1.5.1.1.1 2-Azidopurine nucleosides ....................................................... 26

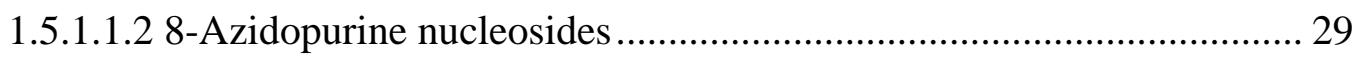

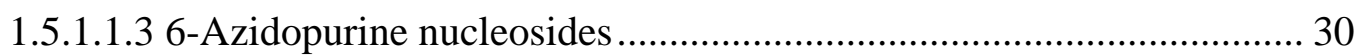

1.5.1.2 Synthesis of azido modified pyrimidine nucleosides.............................. 32

1.5.1.2.1 4-Azidopyrimidine nucleosides...................................................... 32

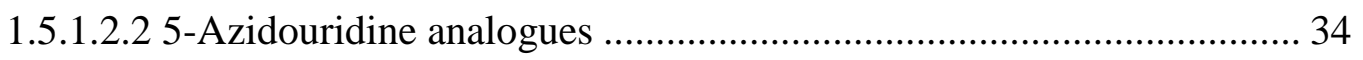

1.5.1.2.3 5-Azidomethyl-2'-deoxyuridine analogues .................................... 35

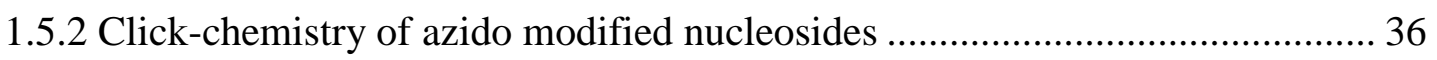

1.5.2.1 Click-chemistry of azido modified purine nucleosides ........................... 36

1.5.2.2 Click-chemistry of azido modified pyrimidine nucleosides .................... 40

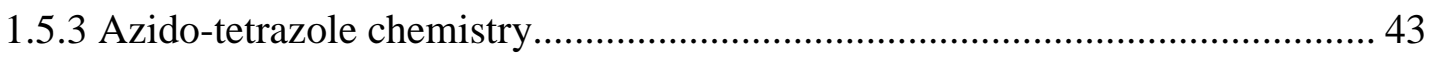

1.5.3.1 Azido/tetrazole tautomerization of 4-azidopyrimidine nucleosides ......... 45

1.5.3.2 Azido/tetrazole tautomerization of azidopurine nucleosides ................... 47

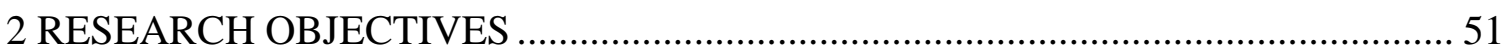




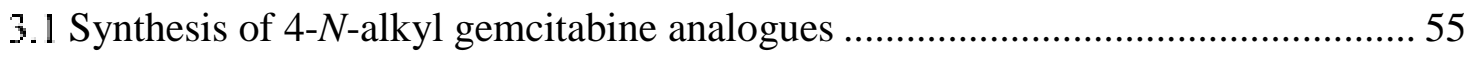

3.1.1 4- $\mathrm{N}$-Alkylgemcitabine with $\beta$-keto tosylate moiety ........................................ 55

3.I.I.I 4- $N$-Alkyl $\beta$-keto tosylate gemcitabine analogue: Synthesis ..................... 56

3.1.1.2 4- $N$-Alkyl $\beta$-keto tosylate gemcitabine analogue: Attempted fluorination 58

3.1.2 4- $\mathrm{N}$-Alkyl gemcitabine conjugated to NOTA ${ }^{68} \mathrm{Ga}$ chelators........................... 59

3.1.2.1 4- $\mathrm{N}$-Alkyl gemcitabine NOTA ${ }^{68} \mathrm{Ga}$ chelators: Synthesis .......................... 60

3.1.2.2 4- $N$-Alkyl gemcitabine NOTA ${ }^{68} \mathrm{Ga}$ chelators: Labeling and Imaging ..... 64

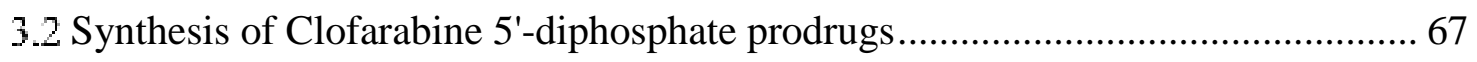

3.2.1 Clofarabine 5'-diphosphate prodrugs: significance and specifications.............. 67

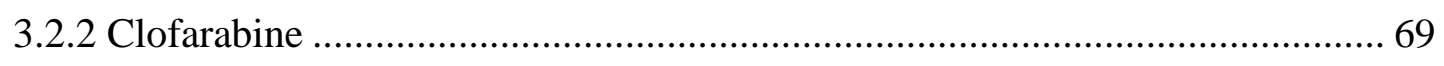

3.2.2.1 Linear synthesis of clofarabine from guanosine ......................................... 70

3.2.2.2 Synthesis of clofarabine by sugar/base coupling ..................................... 72

3.2.3 Synthesis of clofarabine 5'-monophosphate ................................................... 73

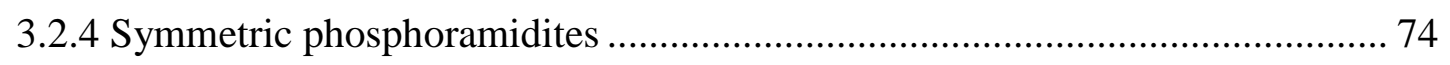

3.2.5 Model studies: adenosine 5'-diphosphate prodrug.......................................... 76

3.2.6 Attempted synthesis of clofarabine 5'-diphosphate ProTides ........................... 81

3.3 Synthesis of azido-modified nucleosides for elucidation of structure of aminyl radicals

3.3.1 Synthesis of 2-azidopurine nucleosides for elucidation of the guanyl aminyl radical 83

3.3.1.1 Synthesis of 2-azidoinosine analogues from 2-azidoadenosine analogues 84

3.3.2 Synthesis of 6-azidopurine nucleoside to study adenylyl aminyl radical......... 90

3.3.3 Synthesis of 4-azidopyrimidine nucleosides to study cytidyl aminyl radical .. 93

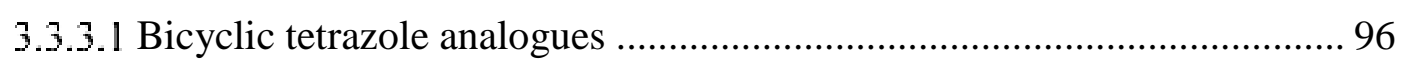

3.3.4 ESR studies of radicals generated from azido-modified nucleosides............... 98

3.3.4.1 From azido-modified pyrimidine nucleosides .......................................... 98

3.3.4.1.1 Proposed conversion $\mathrm{RN}_{3}{ }^{\bullet}$ to $\mathrm{C}^{\prime}{ }^{\bullet}$ sugar radical via UV

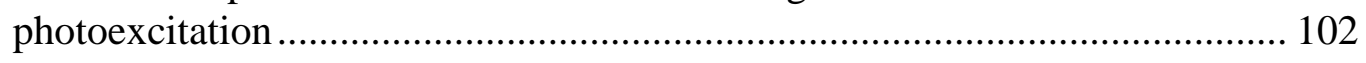

3.3.4.2 From azido-modified purine nucleosides (2- $\mathrm{N}_{3}$-dGuo) ............................ 107

3.3.4.2.1 Photoexcitation of $\mathrm{RN}_{3}{ }^{\circ}$ from 2-Az-dGuo leads to the formation of

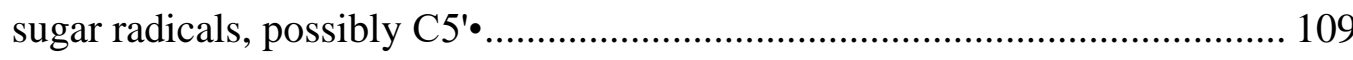

3.3.5 Biological evaluation of azido-modified nucleosides in vivo ......................... 113

3.3.1 Synthesis of 4-azido-2'-deoxy-5',5"-dideuteropyrimidine nucleoside to study formation of $\mathrm{C}^{\prime}$ c centered radical .................................................................... 115

3.3.1.1 Synthesis of 3',5'-di- $O$-acetyl-2'-deoxyuridine for model studies............ 116 
3.3.1.2 Synthesis of 3',5'-di- $O$-acetyl-2'-deoxy 5',5'-dideuterouridine and its 4azido and 4-tetrazolo derivatives

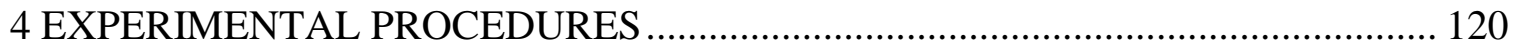

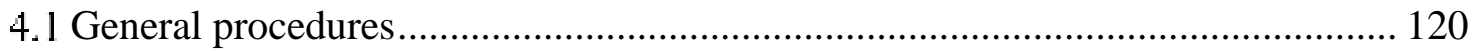

4.2 Synthesis and evaluation of 4- $\mathrm{N}$-Alkyl gemcitabine analogues........................... 120

4.2.1 Synthesis of 4- $\mathrm{N}$-alkylgemcitabine with terminal fluoromethyl ketone......... 120

4.2.2 Synthesis of 4- $\mathrm{N}$-alkylgemcitabine NOTA ${ }^{68} \mathrm{Ga}$ chelators............................. 127

$4.2 .2 .1{ }^{68} \mathrm{Ga}$ radiolabeling and in vivo imaging of $4-\mathrm{N}$-alkyl gemcitabine-SCN-

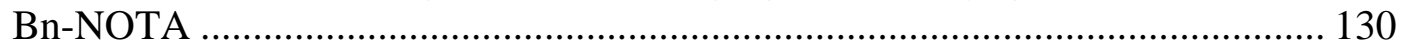

4.3 Synthesis of adenosine 5'-diphosphate prodrug (AdeDiPPro) ……..................... 132

4.4 Synthesis of azido nucleoside analogues ........................................................ 135

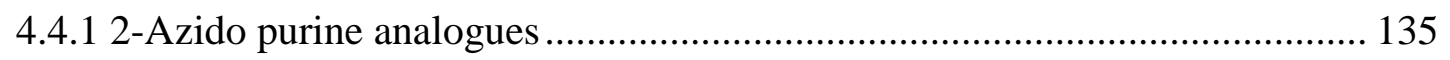

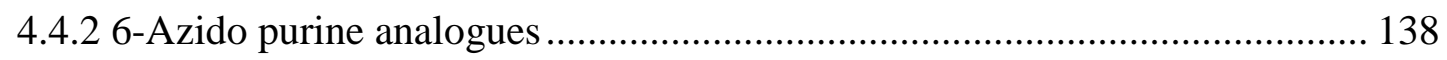

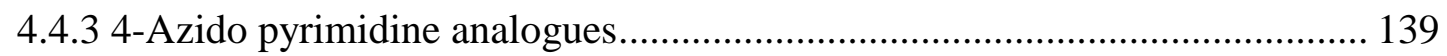

4.5 ESR studies of azido-modified nucleosides ................................................ 140

4.5.1 Sample preparation and methodology …………...................................... 140

4.6 In vivo biological evaluation of azido-modified nucleosides................................. 143

4.7 Synthesis of 3',5'-di-O-acetyl-4-azido-5',5'-dideutero-2'-deoxyuridine ….......... 143

5 CONCLUSION

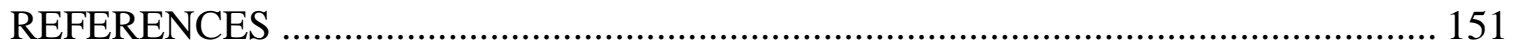

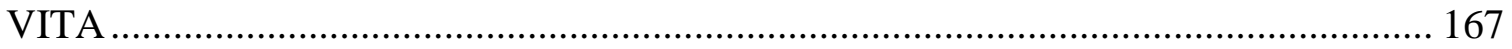




\section{LIST OF TABLES}

TABLE

PAGE

Table 1. ${ }^{1} \mathrm{H}$ NMR Chemical Shifts of Azido/Tetrazole (A/T) Tautomers of 2azidoadenine nucleosides

Table 2. Conditions for synthesis of 6-azido substituted nucleosides from inosine

Table 3. Conditions for the click-reactions of 6-azidopurine nucleosides...................... 39

Table 4. ${ }^{1} \mathrm{H}$ NMR Chemical Shifts for Azido/Tetrazole Tautomers of 2-azidopurine nucleosides in Deuterated Solvents

Table 5. ${ }^{1} \mathrm{H}$ NMR Chemical Shifts for Azido/Tetrazole Tautomers of 6-azidopurine nucleosides in Deuterated Solvents

Table 6. Labeling values for 4- $N$-alkylgemcitabine-NOTA ligand 9 with ${ }^{68} \mathrm{Ga}$ 66 


\section{LIST OF FIGURES}

FIGURE

PAGE

Figure 1. Selected examples of nucleoside analogue drugs 1

Figure 2. Mode of inhibition of ribonucleotide reductase by nucleoside analogues ${ }^{11} \ldots \ldots . .2$

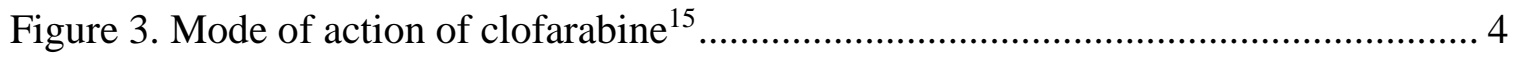

Figure 4. Important example of gemcitabine prodrugs ............................................ 6

Figure 5. Examples of important ProTides/DiPPro analogues reported in literature ......... 7

Figure 6. Proposed in vivo metabolism of McGuigan's ProTides ${ }^{49}$............................... 8

Figure 7. Proposed in vivo metabolism of Meier's DiPPro ${ }^{51}$...................................... 9

Figure 8. Positron annihilation and subsequent detection of photons in PET imaging ${ }^{58} .10$

Figure 9. Important nucleoside- and carbohydrate-based PET imaging radiotracers....... 12

Figure 10. Examples of nucleoside-based PET radiotracers ....................................... 13

Figure 11. Example of c(RGDyK)-SCN-Bn-NOTA chelator with ${ }^{68} \mathrm{Ga}_{\text {-radiolabel }}{ }^{77} \ldots \ldots . .14$

Figure 12. Proposed mechanism of aminyl radical formation in $3^{\prime}-\mathrm{AZT}^{104} \ldots \ldots \ldots \ldots \ldots \ldots . . . . . . .16$

Figure 13. Proposed mechanism for formation of $\pi$-aminyl radical and $\sigma$-iminyl radical from AmdU and AvdU. ${ }^{106}$............................................................................. 18

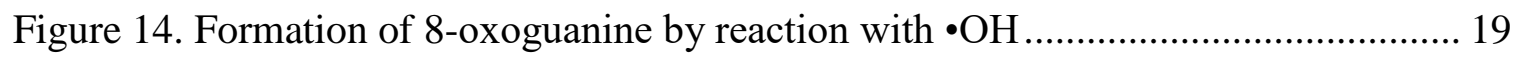

Figure 15. Chatgilialoglu's proposed $\bullet \mathrm{OH}$ oxidation mechanism of guanine base ${ }^{115} \ldots \ldots .20$

Figure 16. Formation of aminyl radical from hydrazines ${ }^{120}$ and hydrazones ${ }^{119} \ldots \ldots \ldots \ldots . . . .22$

Figure 17. Common byproducts of pyrimidine oxidation under anoxic conditions ......... 23

Figure 18. Addition of hydroxy anion to one-electron oxidized thymidine ${ }^{110} \ldots \ldots \ldots \ldots \ldots . . . . . .23$ 
Figure 19. Cytidine syn aminyl radical tautomerization to iminyl radical ${ }^{125}$ 24

Figure 20. Common application of azido-modified nucleoside analogues..................... 25

Figure 21. Azido-tetrazole tautomer of 6-azidopurine nucleosides ............................... 32

Figure 22. AdU and AmdU images after incubation with AlexaFluor 488 alkyne $^{161} \ldots . . .42$

Figure 23. POM-AmdU images after incubation with AlexaFluor 594 alkyne ${ }^{183}$........... 42

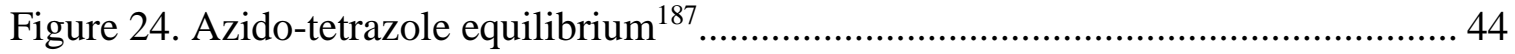

Figure 25. Azido-tetrazole equilibria in 2-azidopurines and 4-azidopyrimidines ${ }^{194,196} \ldots 45$

Figure 26. Azido-tetrazole tautomerization in 4-azidopyrimidine nucleosides............... 46

Figure 27. Synthesis of phosphoramidite precursor for the solid phase synthesis

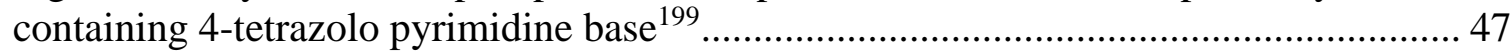

Figure 28. Azido-tetrazole isomers in 2-, 6-, 8-azido substituted purine nucleosides...... 48

Figure 29. Proposed 4- $N$-alkyl gemcitabine analogues suitable for radiolabeling .......... 51

Figure 30. Proposed structure of clofarabine 5'-diphosphate ProTides .......................... 53

Figure 31. Structure of proposed azido modified nucleosides for study of aminyl radical

Figure 32. Azido-Tetrazole equilibrium in 4-azidopyrimidine nucleosides ................... 54

Figure 33. 4- $N$-Alkylgemcitabine derivative bearing $\beta$-keto tosylate moiety for

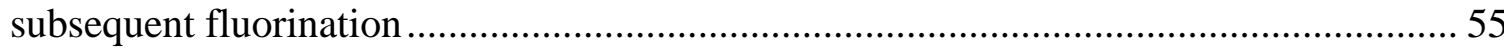

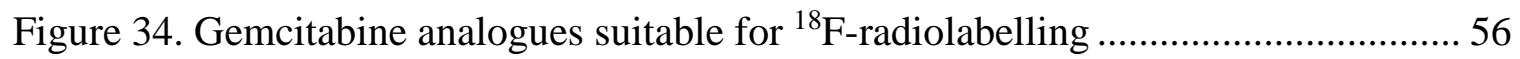

Figure 35. 4- $N$-alkylgemcitabine-NOTA analogue for ${ }^{68}$ Ga-radiolabeling ..................... 60

Figure 36. HPLC purification of conjugate 65 from the crude reaction mixture ............ 62

Figure 37. Stability studies of compound 65 after 2 weeks in a $-20^{\circ} \mathrm{C}$ fridge................. 63 
Figure 38. Stability studies of solid 65 after 2 weeks on bench

Figure 39. TLC for complexation of NOTA-4- $N$-alkylgemcitabine 65 with ${ }^{68} \mathrm{Ga}$. Free $\left[{ }^{68} \mathrm{Ga}\right]{ }^{3+}$ (lane A) and $\left[{ }^{68} \mathrm{Ga}\right]$-complexed-68 (lane B)

Figure 40. HPLC purification of $\left[{ }^{68} \mathrm{Ga}\right]-68$ from minor impurities after Sep-Pak purification

Figure 41. BALB/c maximum intensity projection (MIP) $\mu \mathrm{PET} / \mathrm{CT}$ mice images at onehour post injection showing rapid renal clearance

Figure 42. Clofarabine diphosphate and proposed Clofarabine diphosphate prodrugs. ... 68

Figure 43. HPLC purification of clofarabine 5'-monophosphate 74

Figure 44. ${ }^{31} \mathrm{P}$ NMR of crude bis(benzoyloxybenzyl)phosphoramidite 76

Figure 45. HPLC purification of DiPPro 87 using $\mathrm{CH}_{3} \mathrm{CN} / \mathrm{H}_{2} \mathrm{O}$ system. 78

Figure 46. ${ }^{31} \mathrm{P}$ NMR analysis of the formation of adenosine DiPPro before and after oxidation.

Figure 47. Proposed mechanism for the formation of aminyl radical in 2-azido-2'deoxyinosine upon irradiation with solvated electrons.

Figure 48. Retrosynthetic analysis of 2-azido-2'-deoxyinosine and 2-azidoinosine for the study of the elusive guanylyl aminyl radical

Figure 49. Proposed mechanism of aminyl radical formation from 6-azidopurine 2'deoxynucleoside analogue

Figure 50. Retrosynthesis of 6-azidopurine 2'-deoxynucleoside from 2'-deoxyinsoine ... 91

Figure 51. Proposed mechanism for the formation of aminyl radical followed by tautomerization to iminyl radical in 4-azidopyrimidine analogues .....

Figure 52. Retrosynthesis for formation of 4-azidopyridimidine from 2'-deoxyuridine .. 94

Figure 53. ESR spectra obtained from compounds 112 and 113 via irradiation with radiation-produced prehydrated electrons. 
Figure 54. Theoretical calculations of azide anion radical (RN3•-) in 1-methylcytosine.

Figure 55. Theoretical calculations of azide anion radical (RN3•-) in N3-protonated 1methylcytosine.

Figure 56. ESR spectra obtained after $\gamma$-irradiation and subsequent UV photoexcitation of the tetrazole 113

Figure 57. ESR spectrum of aminyl radical formed upon radiation-produced electron addition to 5-azidouridine (5-AzU)

Figure 58. Comparison of EPR spectra acquired for 2-azido-2'-deoxyinosine 89, 4tetrazolo-2'-deoxyuridine 113 , and 2'-deoxy- $N^{1}$-methylguanosine.

Figure 59. ESR spectra obtained from a 2-Az-dGuo 89 after radiation-produced electron addition and UV photoexcitation

Figure 60. Survival data of MDA-MB-231 cell lines after incubation with azido modified nucleosides and gamma radiation. Data is shown for survival rates after 24 and 48 hours.

Figure 61. Survival data of MCF7 cell lines after incubation with azido modified nucleosides and gamma radiation. Data is shown for survival rates after 24 and 48 hours

Figure 62. Survival data of U87 cell lines after incubation with azido modified nucleosides and gamma radiation. Data is shown for survival rates after 24 and 48 hours.

Figure 63. Retrosynthetic analysis for the synthesis of 4-azido-2'-deoxy-5',5"dideuteropyrimidine nucleoside 


\section{LIST OF SCHEMES}

SCHEME

PAGE

Scheme 1. Synthesis of 2-azido-2'-deoxyinosine from 2-fluoro-2'-deoxyinosine

Scheme 2. Approaches towards synthesis of 2-azidoadenosine and 2-azido-2'deoxyadenosine 28

Scheme 3. Synthesis of 2-azidopurine nucleoside and its tetrazole tautomer 28

Scheme 4. Synthesis of 2-azido- $N^{6}$-methyladenosine from 2-iodo- $N^{6}$-methyladenosine and its equilibrium with its corresponding tetrazole.....

Scheme 5. Synthesis of 8-azidoadenosine monomer for the synthesis of ODN via solidphase synthesis

Scheme 6. Synthesis of 6-azidopurine nucleosides from inosine derivatives

Scheme 7. Alternative synthesis of 6-azidopurine nucleosides from inosine derivatives

¿Scheme 8. Synthesis of protected 4-tetrazolopyrimidine nucleoside ${ }^{150}$

Scheme 9. Synthesis of 4-azido and 4- tetrazolopyrimidine nucleoside from protected arabino uridine ${ }^{151}$

Scheme 10. Gourdain et al. synthesis of 5-azido-2'-deoxyuridine ${ }^{152}$

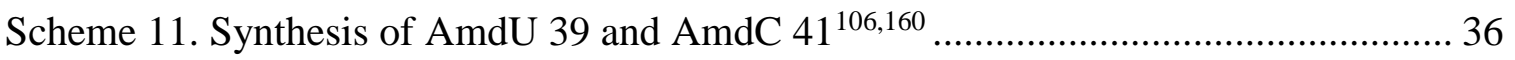

Scheme 12. Synthesis of 2-triazole substituted purine nucleosides .............................. 37

Scheme 13. Regioselective synthesis of 2-triazole substituted adenine nucleosides from 2,6-diazido precursors

Scheme 14. Synthesis of 2,6-ditriazole substituted purine nucleosides followed by regioselective amination

Scheme 15. Azido/tetrazole tautomerization of 6-azidopurine nucleosides and their reactivity towards click- reactions 
Scheme 16. SPAAC "click" reactions between azido nucleosides and cyclooctyne........ 40

Scheme 17. Synthesis of 5-trans-cyclooctyne dUTP analogues ${ }^{184}$................................. 43

Scheme 18. Synthesis of $4-N$-alkylgemcitabine with terminal alkene ........................... 57

Scheme 19. Synthesis of $4-N$-alkyl gemcitabine bearing $\beta$-keto tosylate moiety............. 58

Scheme 20. Fluorination of $4-N$-alkyl $\beta$-keto tosylate gemcitabine analogue .................. 59

Scheme 21. Preparation of the gemcitabine-NOTA conjugate with 4- $N$-alkyl linker ${ }^{207} . .61$

Scheme 22. Labeling of 4- $N$-alkylgemcitabine-NOTA conjugate with Gallium .............. 64

Scheme 23. Labeling of 4- $N$-alkylgemcitabine-NOTA conjugate with ${ }^{68} \mathrm{Ga} \ldots \ldots \ldots \ldots \ldots \ldots \ldots . . . . .65$

Scheme 24. Overall route to clofarabine 5'-diphosphate prodrugs ................................... 69

Scheme 25. Synthesis of protected 6-chloroadenosine from guanosine ........................... 70

Scheme 26. Transformation of 6-chloro-2-aminopurine nucleoside to 2-

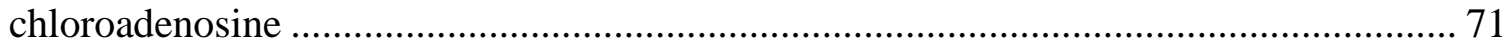

Scheme 27. Attempted synthesis of clofarabine from 2-chloroadenosine........................ 72

Scheme 28. Synthesis of clofarabine by coupling of 2-deoxy-1- $\alpha$-bromo-2- $\beta$-fluoro3,5-di-O-benzoyl- $\beta$-ribofuranose to 2-chloroadenine ...................................................... 73

Scheme 29. Phosphorylation of clofarabine using Yoshikawa method............................ 74

Scheme 30. Synthesis of bis(benzoyloxybenzyl) phosphoramidite .................................. 75

Scheme 31. Synthesis of adenosine 5'-monophosphate triethylammonium salt .............. 77

Scheme 32. Coupling of bis(benzoyloxybenzyl) phosphoramidite to adenosine 5'monophosphate ………......................................................................................... 79

Scheme 33. Attempted coupling of symmetric phosphoramidite to clofarabine 5'monophosphate 
Scheme 34. Conversion of monophosphate counterions in clofarabine 5'monophosphate 82

Scheme 35. Improved synthesis of clofarabine 5'-diphosphate prodrugs......................... 82

Scheme 36. Synthesis of 2-azido-2'-deoxyadenosine from 2'-deoxyguanosine ............... 86

Scheme 37. Synthesis of 2-azido-2'-deoxyinosine from 2-azido-2'-deoxyadenosine....... 86

Scheme 38. Alternative synthesis of 2-azido-2'-deoxyinosine from 2'-deoxyguanosine . 88

Scheme 39. Synthesis of 2-azidoadenosine from 6-chloro-2-amino protected purine ..... 89

Scheme 40. Synthesis of 2-azidoguanosine from 2-azidoadenosine ............................... 89

Scheme 41. Tautomerization of 2-azidoadenosine analogues .......................................... 90

Scheme 42. Routes for the synthesis of 6-azidopurine 2'-deoxynucleoside from 2'deoxyinosine

Scheme 43. Tautomerization of 6-azidopurine derivatives 92

Scheme 44. Attempted deacetylation of 6-azidopurine 2'-deoxynucleoside caused complete decompostion

Scheme 45. Synthesis of 4-azidopyrimidine 2'-deoxy nucleoside

Scheme 46. Deprotection of 3',5'-di- $O$-acetyl protected 4-azidopyrimidine nucleoside giving 4-tetrazolopyrimidine nucleoside product

Scheme 47. Synthesis of 4-azidopyrimidine and 4-tetrazolopyrimidine nucleosides ...... 98

Scheme 48. Reaction of tetrazole 113 and azido 112 with radiation produced electrons to form RN3 $\bullet$.

Scheme 49. Proposed mechanism for the formation of C5'-sugar radical upon photoexcitation of the $\mathrm{RN}_{3}{ }^{-}$in tetrazole 113 and azido 112 .

Scheme 50. Proposed mechanism for the formation of C5' and C3' centered carbon radicals upon UV-photoexcitation of the RN3 •- in 2-azidoguanosine derivatives. 
Scheme 51. Synthesis of 1,3,5-tri- $O$-acetyl-2'-deoxyribose for coupling reaction......... 117

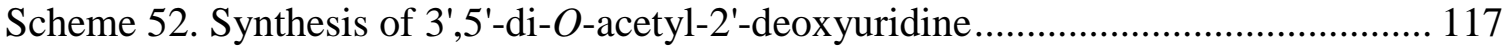

Scheme 53. Synthesis of 1,3,5-tri- $O$-acetyl-2-deoxy-5,5'-dideuteroribose for

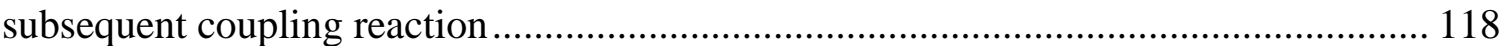

Scheme 54. Synthesis of 3',5'-di- $O$-acetyl-2'-deoxy-5',5"-dideuterouridine .................... 119

Scheme 55. Synthesis of 3',5'-di-O-acetyl-4-azido-5',5''-dideutero-2'-deoxyuridine ... 119 


\title{
ABREVIATION AND ACRONYMS
}

\author{
${ }^{18} \mathrm{~F}-\mathrm{CFA}$ \\ ${ }^{18}$ F-clofarabine \\ ${ }^{18} \mathrm{FDG}$ \\ 2-deoxy-2-[ $\left[{ }^{18} \mathrm{~F}\right]-\mathrm{D}$-glucose \\ ${ }^{18}$ F-FAC \\ 1-(2-deoxy-2- $\left[{ }^{18}\right.$ F]fluoro- $\beta$-D-arabinofuranosyl)cytosine \\ $2^{\prime}-\left[{ }^{18} \mathrm{~F}\right] \mathrm{dFdC}$ \\ ${ }^{18} \mathrm{~F}$-gemcitabine \\ 2-Az-dGuo \\ 2-azido-2'-deoxyinosine \\ 3'-AZT \\ 3'-azido-3'-deoxythymidine \\ $5-\mathrm{BrdU}$ \\ 5-bromo-2'-deoxyuridine \\ 5-IdU \\ 5-iodo-2'-deoxyuridine \\ 8-oxodGuo \\ 8-oxo-7,8-dihydro-2'-deoxyguanosine \\ AdU \\ 5-azido-2'-deoxyuridine \\ AIDS \\ acquired immune deficiency syndrome \\ ALL \\ acute lymphoblastic leukemia \\ AmdC \\ 5-azidomethyl-2'-deoxycytidine \\ AmdU \\ 5-azidomethyl-2'-deoxyuridine \\ AmvC \\ 5-(1-azidovinyl)-2'-deoxycytidine \\ $\mathrm{AmvU}$ \\ 5-(1-azidovinyl)-2'-deoxyuridine \\ ara-A \\ 9-( $\beta$-D-arabinofuranosyl)adenine \\ ara-C \\ 1-( $\beta$-D-arabinofuranosyl)cytosine \\ Ara-C \\ 1-( $\beta$-D-arabinofuranosyl)cytosine \\ $\mathrm{AzU}$ \\ 5-azidouridine \\ $\mathrm{BzCl}$ \\ benzoyl chloride \\ $\mathrm{c}(\mathrm{RGDyK})$ \\ cyclic Arg-Gly-Asp -D-Tyr-Lys




\begin{tabular}{|c|c|}
\hline $\mathrm{CuAAC}$ & Copper(I)-Catalyzed Azide-Alkyne Cycloaddition \\
\hline $\mathrm{dCDA}$ & deoxycytidine deaminase \\
\hline DCI & 4,5-dicyanoimidazole \\
\hline $\mathrm{dCK}$ & deoxycytidine kinase \\
\hline $\mathrm{dCTP}$ & deoxycytidine triphosphate \\
\hline $\mathrm{dFdCTP}$ & gemcitabine 5'-triphosphate \\
\hline $\mathrm{dFdU}$ & 2'-deoxy-2',2'-difluorouracil \\
\hline dGuo & 2'-deoxyguanosine \\
\hline DiPPro & Diphosphate Prodrugs \\
\hline DMAP & 4-dimethylaminopyridine \\
\hline DMF & $N, N$-dimethylformamide \\
\hline dNDPs & deoxynucleoside diphosphates \\
\hline dNTPs & deoxynucleoside triphosphates \\
\hline dsDNA & double stranded DNA \\
\hline EDG & electron donating group \\
\hline EPR & Electron Paramagnetic Resonance \\
\hline ESR & Electron Spin Resonance \\
\hline EWG & electron withdrawing group \\
\hline $\mathrm{F}_{2} \mathrm{CDP}$ & gemcitabine 5'-diphosphate \\
\hline $\mathrm{F}_{2} \mathrm{CMP}$ & gemcitabine 5'-monophosphate \\
\hline FapyAde & 4,6-diamino-5-formamidopyrimidine \\
\hline FapyGua & 2,6-diamino-4-hydroxy-5-formamidopyrimidine \\
\hline G & Gauss \\
\hline
\end{tabular}




\begin{tabular}{|c|c|}
\hline $\mathrm{G}(\mathrm{N} 2-\mathrm{H}) \bullet$ & guanyl aminyl radical \\
\hline Gy & Grey \\
\hline$H$ & external magnetic field \\
\hline $\mathrm{H} 1^{\prime}$ & anomeric proton \\
\hline hCNT3 & human concentrative nucleoside transport protein 3 \\
\hline hENT1 & human equilibrative nucleoside transport protein 1 \\
\hline hENTs & human equilibrative nucleoside transport proteins \\
\hline$h f c$ & isotropic hyperfine coupling constant \\
\hline $\mathrm{HO} \cdot$ & hydroxyl radical \\
\hline HPLC & High-Performance Liquid Chromatography \\
\hline$h v$ & microwave radiation \\
\hline MS & Mass Spectrometry \\
\hline NA & nucleoside analogues \\
\hline NDP kinase & nucleotide diphosphate kinase \\
\hline NMO & $N$-methylmorpholine $N$-oxide \\
\hline NMR & nuclear magnetic resonance \\
\hline NPE & p-nitrophenethyl \\
\hline ODN & oligodeoxynucleotides \\
\hline PET & Positron Emission Tomography \\
\hline ProTides & Nucleotide Prodrugs \\
\hline RNR & ribonucleotide reductase \\
\hline ROS & reactive oxygen species \\
\hline SDG & sterically demanding group \\
\hline
\end{tabular}


SPAAC

TBAF

TEAB

TFFA

TIPBSCl

TLC

TMSOTf

UMP/CMP kinase
Strain-Promoted Azide-Alkyne Cycloaddition

tetra- $n$-butylammonium fluoride

triethylammonium bicarbonate

trifluoroacetic acid

2,4,6-triisopropylbenzenesufonyl chloride

Thin Layer Chromatography

trimethylsilyl trifluoromethanesulfonate

uridine/cytidine monophosphate kinase 


\section{INTRODUCTION}

\subsection{Anti-cancer properties of nucleoside analogues}

Cancer is the second leading cause of death in the United States. Cancer cells display uncontrolled growth and spread across the body causing various health issues, or even death. Because of the increased metabolism of cancer cells compared to normal cells, replication of DNA occurs much more frequently in these cells. Replication of DNA requires nucleotides, the building blocks of DNA, whose precursors are nucleosides. On the basis of the fact that cancer cells require more nucleosides, many nucleoside analog (NA) chemotherapeutic agents have been developed. ${ }^{1}$ These NAs mimic regular nucleosides in their uptake and metabolism. They enter the cell through different membrane transporters and are phosphorylated into their active nucleoside triphosphate forms. The triphosphate form of these drugs can be incorporated onto DNA, where their small modifications cause strand breakage and ultimately cell death. ${ }^{2}$

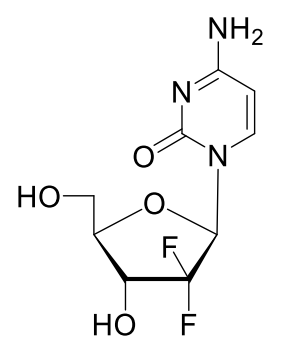

Gemcitabine

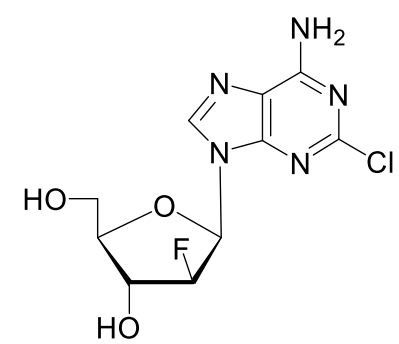

Clofarabine

Figure 1. Selected examples of nucleoside analogue drugs

\subsubsection{Gemcitabine: biological activity}

Gemcitabine $\left(2^{\prime}, 2^{\prime}\right.$-difluoro-2'-deoxycytidine, dFdC, Figure 1) is a chemotherapeutic cytidine analogue synthesized by Hertel $e t a .^{3}$ that is used in the treatment of non-small cell lung, pancreatic, bladder, and breast cancer. ${ }^{4,5}$ Gemcitabine is a hydrophilic drug, and 
requires human equilibrative nucleoside transport proteins (hENTs) to be taken through the cell membrane. ${ }^{4}$ It is then activated in the cell by monophosphorylation with deoxycytidine kinase (dCK) into its monophosphate form. ${ }^{6,7}$ Gemcitabine 5'-monophosphate (dFdCMP) is further phosphorylated by uridine/cytidine monophosphate kinase (UMP/CMP kinase). ${ }^{4}$ Then, dFdCDP is phosphorylated to gemcitabine 5'-triphosphate (dFdCTP) by nucleotide diphosphate kinase (NDP kinase) and can serve as a substrate for DNA incorporation. Once incorporated, gemcitabine causes chain termination during replication and repair processes, thus triggering apoptosis. ${ }^{4}$

Gemcitabine 5'-diphosphate (dFdCDP) serves as a potent inhibitor of both subunits of ribonucleotide reductase (RNR; Figure 2), the enzyme that is responsible for converting ribonucleoside diphosphates into 2'-deoxyribonucleoside diphosphates. ${ }^{8}$ By inhibiting RNR, gemcitabine decreases the bioavailability of naturally occurring deoxynucleoside diphosphates (dNDPs), and subsequently deoxynucleoside triphosphates (dNTPs) in the cell, furthering gemcitabine's ability to compete with dCTP for incorporation into DNA. ${ }^{9}$ Because of the depletion of dCTP pools in the cell, feedback inhibition of dCK is disrupted, which allows further monophosphorylation of gemcitabine. The increasing pools of gemcitabine 5'-monophosphate becomes highly toxic for cancer cells that are replicating rapidly and require a large amount of nucleotide building blocks for DNA synthesis. ${ }^{10}$

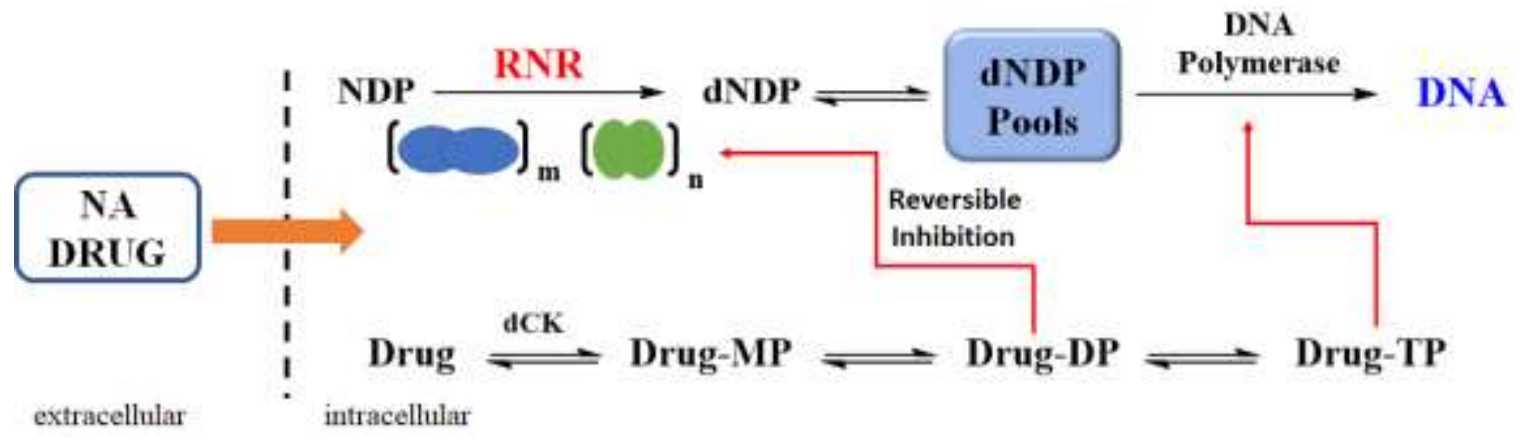

Figure 2. Mode of inhibition of ribonucleotide reductase by nucleoside analogues ${ }^{11}$ 
Although gemcitabine is quite successful in treating certain tumors, it does experience some limitations in vivo in terms of metabolism and cytotoxicity. For example, gemcitabine is significantly hydrophilic, causing its rapid excretion once administered and it is inactive in the body until it can be mono-, di-, and tri-phosphorylated to serve as a substrate for DNA incorporation. The rate-limiting step in its metabolism is its monophosphorylation from gemcitabine into gemcitabine 5'-monophosphate by deoxycytidine kinase (dCK). Gemcitabine 5'-monophosphate can also undergo dephosphorylation by 5'-nucleotidases back into the inactive parent drug. ${ }^{6,7}$ Finally, gemcitabine can be highly toxic to normal cells as a result of its rapid deamination into its toxic 2'-deoxy-2',2'-difluorouracil (dFdU) derivative by deoxycytidine deaminase (dCDA). ${ }^{6,12,13}$

\subsubsection{Clofarabine: biological activity}

Clofarabine [2-chloro-9-(2-deoxy-2-fluoro- $\beta$-D-arabinofuranosyl)adenine, Cl-F-araA, Figure 1] is an adenosine analogue that is used for the treatment of pediatric acute lymphoblastic leukemia (ALL). Clofarabine was synthesized by Montgomery et al. ${ }^{14}$ in order to prevent the dose toxicity found in similar anticancer drugs fludarabine phosphate (9- $\beta$-D-arabinofuranosyl-2-fluoroadenine-5'-O-phosphate) and cladribine (2-chloro-2'deoxyadenosine). Toxicity of fludarabine phosphate and cladribine was found to be the result of their susceptibility to glyosidic bond cleavage. ${ }^{15}$ Presence of the fluorine in arabino configuration at the 2'-position of sugar moiety prevents formation of inactive and/or toxic purine derivatives, while the presence of chlorine at 2-position of adenine base prevents deamination by adenosine deaminase. ${ }^{14}$

Entrance of clofarabine into the cell occurs mainly by human equilibrative nucleoside transport protein $1(\mathrm{hENT1})$ and human concentrative nucleoside transport protein 3 
(hCNT3), although, at high concentration and long exposure times, it can undergo passive diffusion through lipid membranes. ${ }^{15,16}$ Clofarabine is a better substrate for dCK than $2^{\prime}-$ deoxycytidine, and thus is activated in the nucleus through monophosphorylation by the kinase. ${ }^{17}$ Clofarabine is further phosphorylated by purine nucleotide monophosphate kinase into its diphosphate form, and then triphosphorylated for incorporation onto DNA (Figure 3). ${ }^{15}$ Clofarabine 5'-triphosphate is also an RNR inhibitor, and like gemcitabine, lowers the availability of naturally occurring dNTPs, making its incorporation much more rapid. ${ }^{11,18,19}$

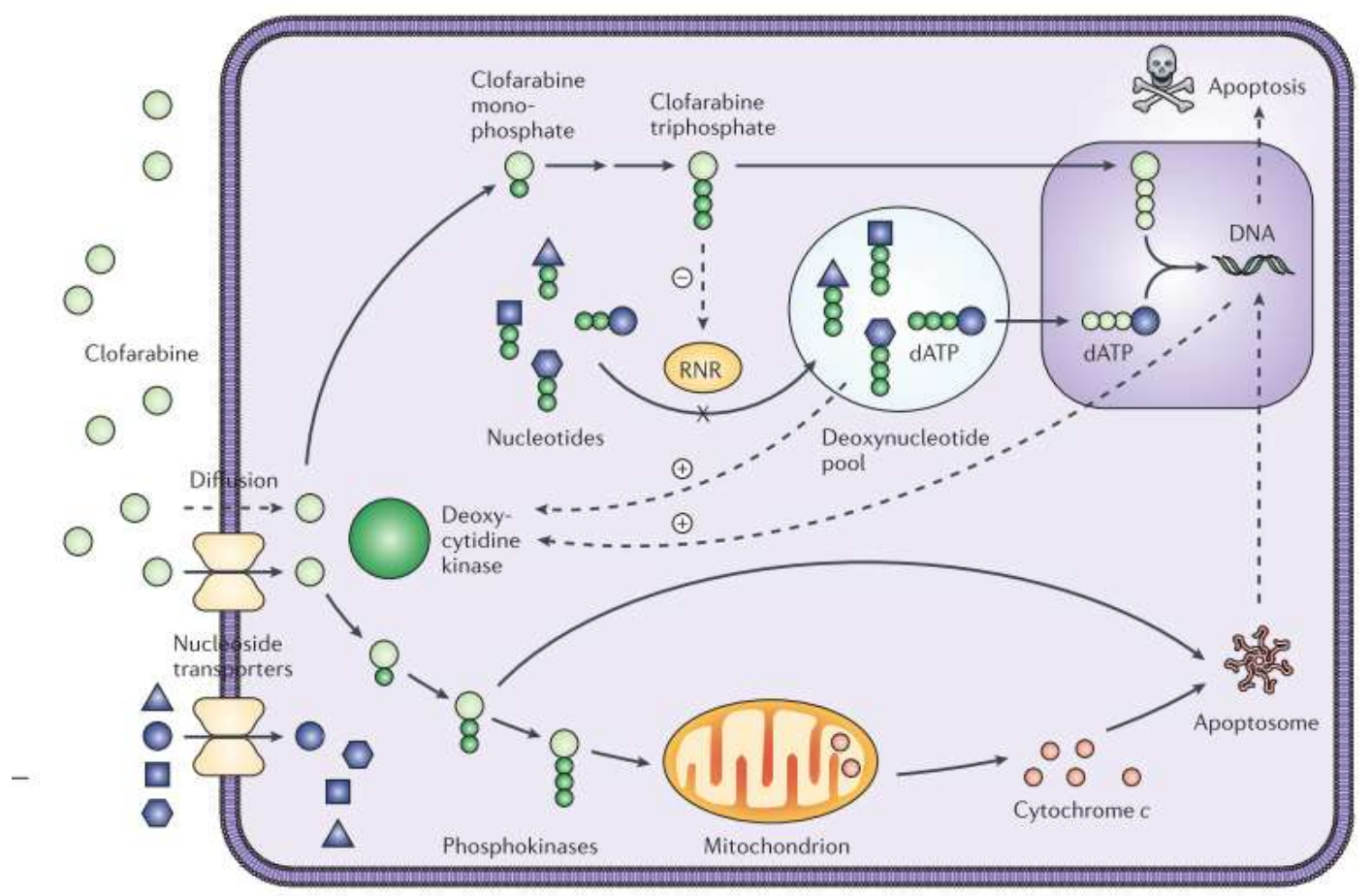

Figure 3. Mode of action of clofarabine ${ }^{15}$

Clofarabine, like gemcitabine, has some limitations in terms of metabolism and cytotoxicity. Unlike fludarabine and other common NA drugs that experience rate-limiting monophosphorylation by $\mathrm{dCK}$, clofarabine undergoes rate-limiting phosphorylation from clofarabine 5'-monophophate to clofarabine 5'-diphosphate by purine nucleotide 
monophosphate kinase. ${ }^{15,20}$ Thus, accumulation of clofarabine 5'-monophosphate which can be incorporated onto DNA and causes some cytotoxicity, but slows the formation of clofarabine 5'-triphosphate which is required for RNR inhibition and the self-potentiating mechanism which increases its anti-cancer activity. ${ }^{21}$ Moreover, clofarabine 5'monophosphate can be dephosphorylated back to the inactive parent metabolite by 5'nucleotidases present in the cell. ${ }^{15,20,22,23}$

\subsection{Nucleoside prodrugs}

Prodrugs are derivatives of parent drugs that can be transformed chemically or enzymatically in vivo to release the parent drug. ${ }^{24}$ The main aim of prodrugs is to improve the physicochemical properties of the parent drug. Because of their high polarity, NA drugs usually suffer from poor bioavailability and low intestinal permeability. Prodrugs, then, are employed to increase the lipophilicity of these drugs, which allows facilitated transport into the cells (often without the need for nucleoside transporters), improve oral absorption, or target specific tissues. ${ }^{25-27}$

Several gemcitabine prodrugs have been developed through hydrolyzable acyl modifications on the exocyclic 4- $N$-amine or 5'-hydroxyl groups, to combat its rapid deamination and excretion. ${ }^{28-33}$ Examples of 4-N-acyl modified gemcitabine include LY2334737 $\mathbf{A}^{30}$ and squalenoyl $\mathbf{B}^{29}$ analogues, which improve uptake and bioavailability of gemcitabine (Figure 4). ${ }^{34,35}$ Furthermore, esterification at the 5' position of gemcitabine accelerates uptake of the drug into tumor cells (e.g., CP-4126; C). ${ }^{31}$ Gemcitabine analogues have also been designed as theragnostic delivery systems with cellular specificity and imaging capabilities; these include $\mathrm{H}$-gemcitabine ${ }^{32}$ and gemcitabine-coumarin-biotin conjugates, ${ }^{36}$ among others. ${ }^{37,38}$ 


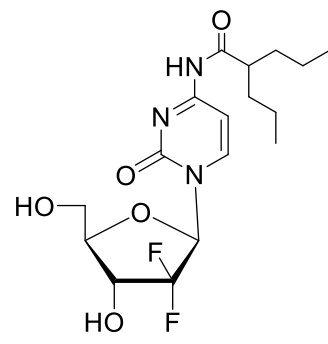

A

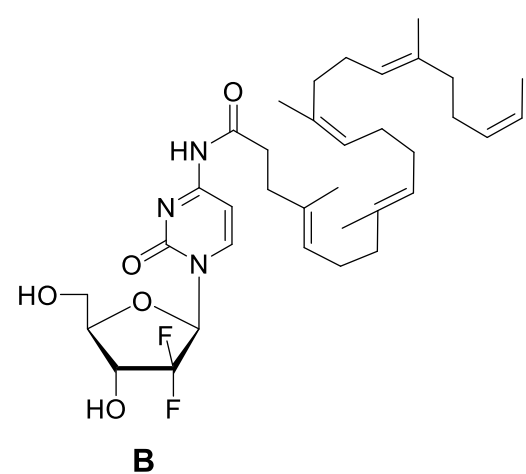

B

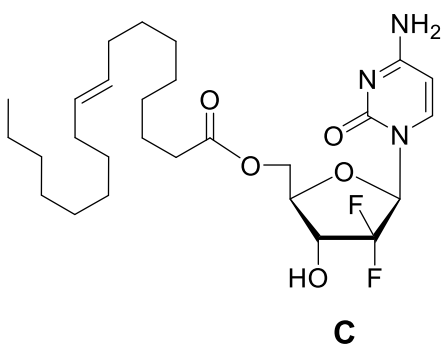

C

Figure 4. Important example of gemcitabine prodrugs

Most nucleoside drugs and prodrugs undergo rate limiting monophosphorylation by $\mathrm{dCK}$. Because of this rate-limiting process, several nucleotide prodrugs (ProTides) have been modified to bypass a phosphorylation event through hydrolysable phosphoramidate modifications at the 5 ' position. ${ }^{39}$ The best example of this highly successful approach, is the development of anti-viral Hepatitis C drug: Sofosbuvir (D). ${ }^{39,40}$ Figure 5 shows some common examples of ProTides synthesized in recent literature for the enhancement of cellular metabolism. 

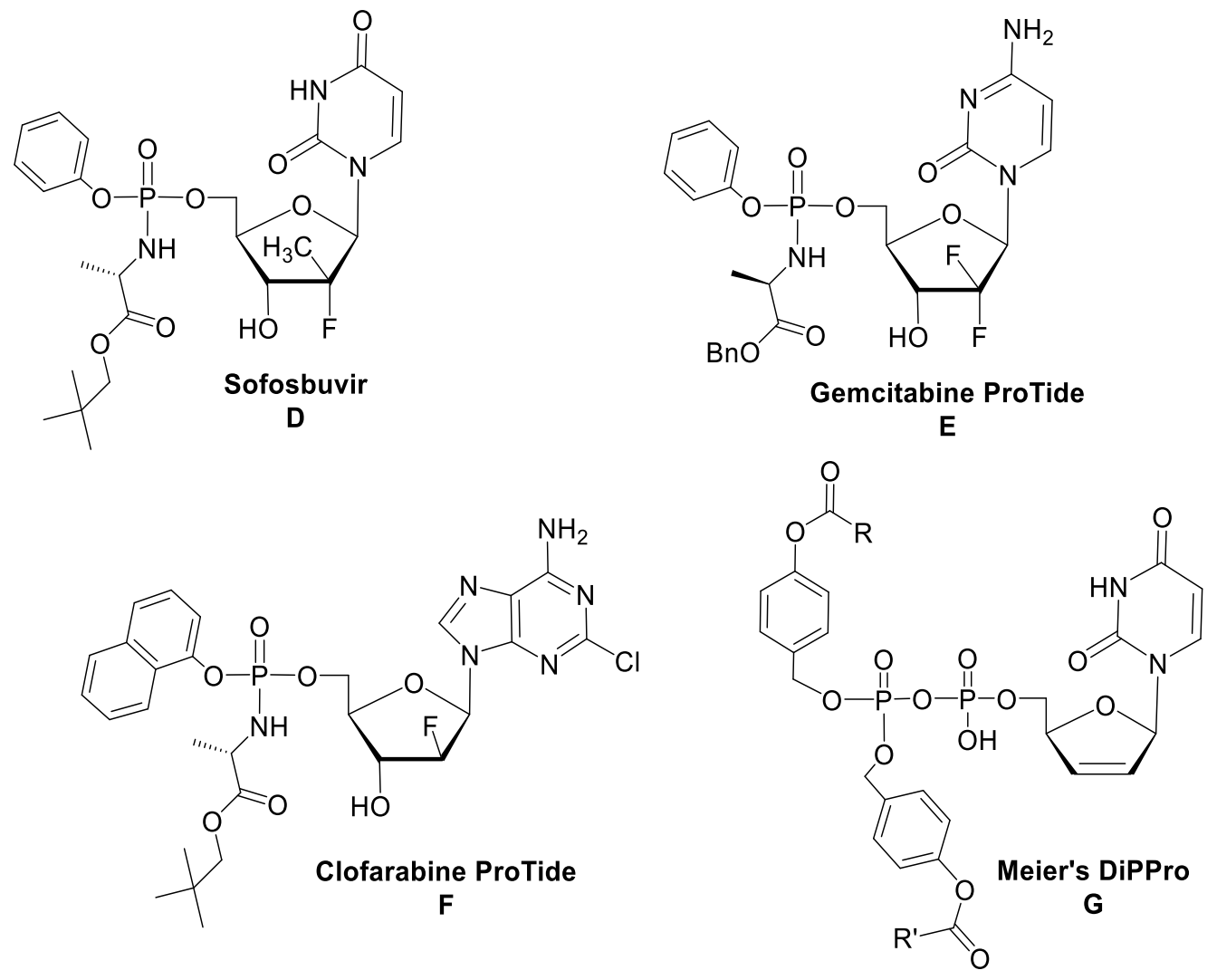

Figure 5. Examples of important ProTides/DiPPro analogues reported in literature

Several types of nucleotide prodrugs have been reported in literature over the last decade. These can be divided into two sub-categories:

1. Dr. McGuigan's phosphoramidate prodrugs (ProTides) ${ }^{41-45}$, and

2. Dr. Meier's diphosphate prodrugs (DiPPro). ${ }^{46,47}$

McGuigan's approach, as seen in sofosbuvir $(\mathbf{D})^{40}$, gemcitabine $(\mathbf{E})^{48}$ and clofarabine $(\mathbf{F})^{45}$ ProTides, involves the addition of a phosphoramidate unit to the 5'-OH of the parent nucleoside drug. These prodrugs are designed to release the 5' monophosphorylated parent drug by a specific in vivo metabolism. The process begins by cleavage of the ester bond of the amino acid derivative modification by action of esterase or carboxypeptidase type enzymes (Figure 6).$^{49}$ Following the cleavage, cyclization spontaneously occurs displacing aryl modification and creating an unstable anhydride ring. The ring can then be opened in 
the presence of water. Finally, a phosphoramidase-type enzyme can hydrolyze the P-N bond, releasing the 5' monophosphorylated parent drug. The unmasked monophosphate form of the drug can then be released to be di- and triphosphorylated in the cell and serve as a substrate for DNA incorporation. ${ }^{49}$

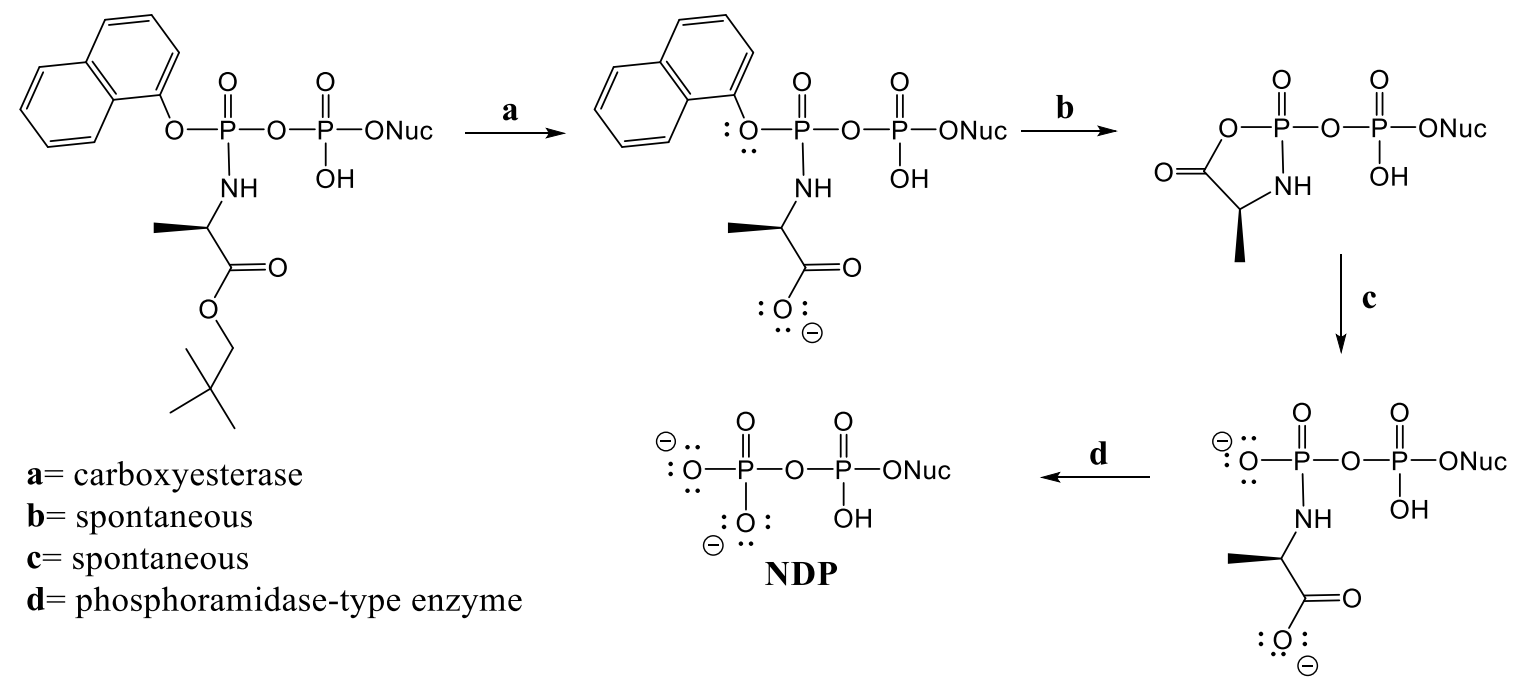

Figure 6. Proposed in vivo metabolism of McGuigan's ProTides ${ }^{49}$

The Meier DiPPro approach, as seen in ProTide G, utilizes a bis(acyloxybenzyl) phosphoramidite unit to couple with nucleoside 5'-monophosphates. Once coupled, a $\mathrm{P}(\mathrm{III})-\mathrm{P}(\mathrm{V})$ bond forms which can be oxidized to give $\mathrm{P}(\mathrm{V})-\mathrm{P}(\mathrm{V})$ bond in which $\beta$ phosphate still contains lipophilic bis(acyloxybenzyl) modifications. ${ }^{50-53}$ Cellular metabolism of the DiPPro begins with esterases cleavage, which allows release of the bis(acyloxybenzyl) moiety as a para-quinone methide byproduct leaving unmasked nucleoside diphosphate (Figure 7). ${ }^{46}$ Although more lipophilic modifications on the ester are preferred for efficiency of uptake, lipophilic modification create a more stable ester bond that undergoes a slower esterase cleavage. Slower esterase cleavage causes release of the entire $\beta$-phosphate unit leaving the monophosphorylated nucleoside analog drug. To avoid the cleavage of the phosphorous anhydride bond, asymmetric bis(acyloxybenzyl) 
modifications are preferred with less lipophilic (shorter carbon chain) modification and one more lipophilic (longer carbon chain) modification on the $\beta$-phosphate. The less lipophilic alkyl ester will then be released quickly, leaving a negative charge on the $\beta$-phosphate, making cleavage of the phosphorous anhydride bond difficult. The more lipophilic bis(acyloxybenzyl) modification can then be metabolized releasing the unmasked nucleoside diphosphate, which can be further phosphorylated to its triphosphate form and serve as a precursor for DNA incorporation. ${ }^{51}$

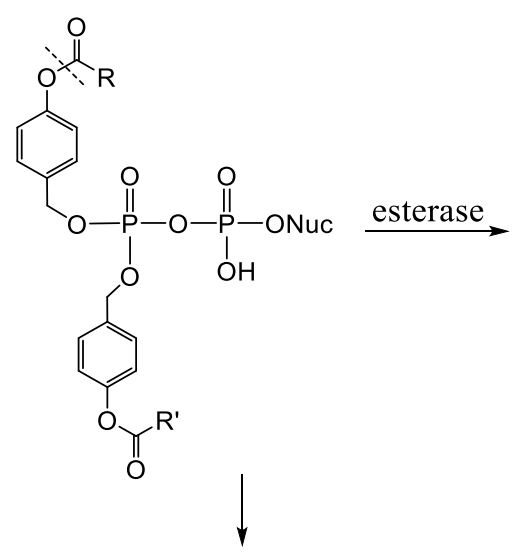

NMP
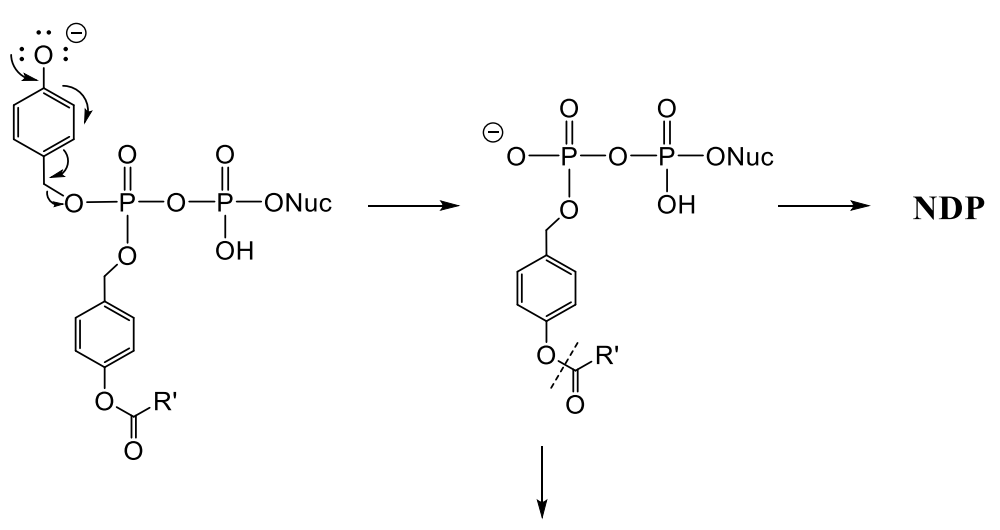

NMP

Figure 7. Proposed in vivo metabolism of Meier's DiPPro ${ }^{51}$

\subsection{Positron Emission Tomography (PET) imaging}

Positron Emission Tomography (PET) is a non-invasive imaging technique which has become increasingly useful for the diagnosis and staging of human disease, as well as, for drug discovery. Positron Emission Tomography is an important tool in medicinal chemistry because of its ability to monitor disease biology and response to treatments. ${ }^{54}$ The PET method utilizes drugs labeled with neutron-deficient isotopes (i.e., radionuclei) that stabilize by converting a proton into a neutron and emitting a positron (such as ${ }^{11} \mathrm{C},{ }^{13} \mathrm{~N}$, ${ }^{15} \mathrm{O}$, or $\left.{ }^{18} \mathrm{~F}\right) .{ }^{55} \mathrm{~A}$ positron is a subatomic particle of equal mass and charge magnitude than an electron, but bears a positive charge. ${ }^{56}$ The emitted positron loses energy as it travels 
through the body and eventually annihilates with an electron. The interaction of the oppositely charged positron and electron causes two gamma rays to discharge at $180^{\circ}$ from one another (i.e., collinear emission). These photons are detected simultaneously, within a few nanoseconds, by a ring detector around the patient. In this way, the specific location of the radiotracer, molecule containing the radionuclei, can be determined and an image formulated. The accumulation of the radiotracer in the body is determined by the nature and metabolism of the radiotracer. ${ }^{56,57}$

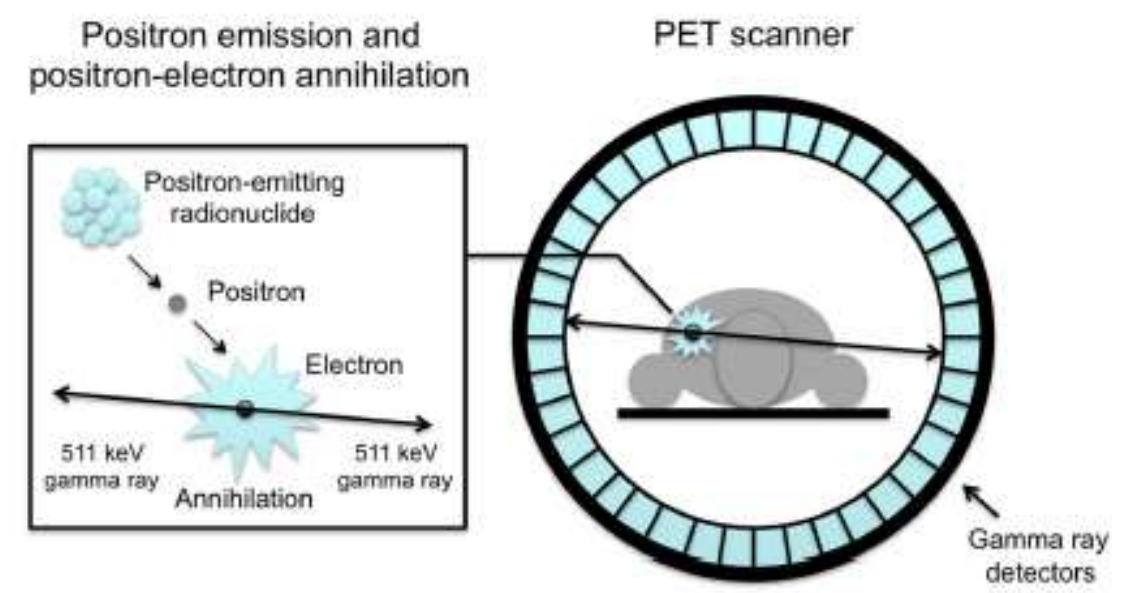

Figure 8. Positron annihilation and subsequent detection of photons in PET imaging 58

One of the most common positron emitting nuclides used for PET imaging is Fluorine$18\left({ }^{18} \mathrm{~F}\right)$ because of its long half-life (109.77 minutes) as compared to other positronemitting radionuclei. The long half-life of ${ }^{18} \mathrm{~F}$ allows sufficient time for the synthesis of radiotracers, as well as, the possibility of transportation outside a radiochemical facility. ${ }^{55}$ The ${ }^{18} \mathrm{~F}$ radioisotope is made in one of two ways:

1. The irradiation of ${ }^{20} \mathrm{Ne}$ with deuterium particles followed by its $\alpha$-decay; with $\mathrm{F}_{2}$ added to keep fluorine oxidized, which allows for use of ${ }^{18} \mathrm{~F}$ directly from the nuclear reactor (cyclotron). ${ }^{59}$ 
2. The irradiation of ${ }^{18} \mathrm{O}$ enriched water with protons in a cyclotron, where the ${ }^{18} \mathrm{~F}$ ion formed can be removed using distillation or a resin column. ${ }^{59}$

The most common radiotracer currently used for PET imaging is 2-deoxy-2- $\left[{ }^{18} \mathrm{~F}\right]-\mathrm{D}$ glucose $\left({ }^{18} \mathrm{FDG}\right.$, Figure 9$)$, which is metabolized similarly to glucose in the body. It is taken into the cell with glucose transporter available in all living cells and phosphorylated irreversibly by hexokinase. However, it cannot be further metabolized because of the fluorine present at the 2-position and remains trapped in the cell. Cells with higher glucose consumption, such as cancer cells, will accumulate ${ }^{18} \mathrm{FDG}$ and create a contrasting image with the surrounding environment. ${ }^{54}$ Unfortunately, ${ }^{18} \mathrm{FDG}$ is metabolized quickly and is not as useful in imaging tumors with slow proliferation or brain tumors, since glucose accumulates in all brain cells as consequence of their high metabolic requirements. ${ }^{59}$

\subsubsection{Overview of PET imaging agents}

\subsubsection{Nucleoside- and carbohydrate-based ${ }^{18}$ F-PET imaging agents}

To monitor specific tumor sites in the body and get accurate data for drug efficacy, several nucleoside-based PET radiotracers have been developed (Figure 9) ${ }^{60}$ Nucleosidebased radiotracers are particularly useful to image cancer growth and treatment because they are involved in DNA synthesis. ${ }^{61}$ These radiotracers are labelled with ${ }^{11} \mathrm{C}$ or ${ }^{18} \mathrm{~F}$ radionuclides and are substrates for key enzymes in DNA synthesis, such as kinases. Most of the known PET imaging radiotracers are designed from pyrimidine nucleoside analogues. ${ }^{61}$ 


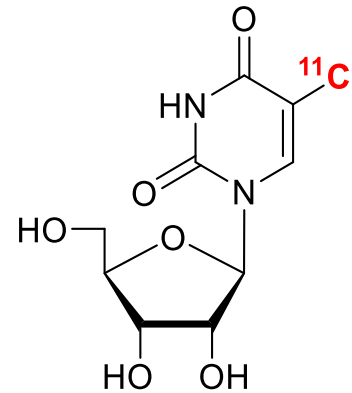

$\left[{ }^{11} \mathrm{C}\right]-\mathrm{FMAU}$

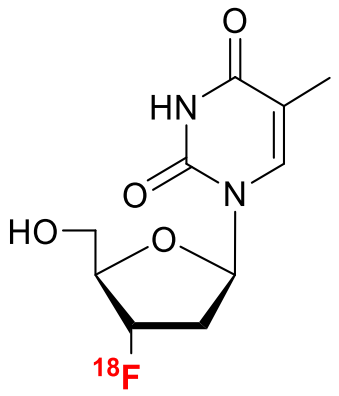

$\left[{ }^{18} \mathrm{~F}\right]-\mathrm{FLT}$

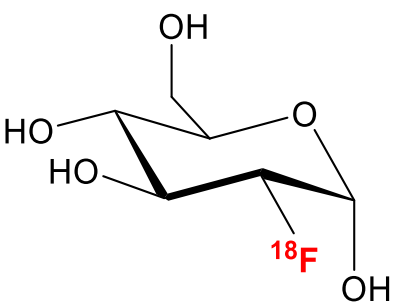

$\left[{ }^{18} \mathrm{~F}\right]$-FDG

Figure 9. Important nucleoside- and carbohydrate-based PET imaging radiotracers

One important example is 1 -(2-deoxy-2- $\left[{ }^{18} \mathrm{~F}\right]$ fluoro- $\beta$-D-arabinofuranosyl)cytosine $\left[{ }^{18} \mathrm{~F}-\mathrm{FAC}\right.$, Figure 10], which was developed as a noninvasive predictor probe of tumor response to gemcitabine and was utilized for PET imaging of dCK activity. ${ }^{62-65}$ The ${ }^{18} \mathrm{~F}$ FAC was developed because the synthesis of gemcitabine involves the incorporation of a geminal difluoro unit in the early synthetic stages, ${ }^{3,66}$ and the preparation of $2^{\prime}-\left[{ }^{18} \mathrm{~F}\right] \mathrm{dFdC}$ still appears to be not feasible mainly because of the short half-life of ${ }^{18} \mathrm{~F}$ isotope ${ }^{67}$ Although FAC generally follows the known ex vivo biodistribution of gemcitabine, it is missing the geminal difluoromethylene unit at $\mathrm{C} 2$ ' which is critical for the anticancer properties of gemcitabine, its inhibitory activity of RNR, ${ }^{9}$ and other physicochemical properties. ${ }^{68-70}$

Recent literature showed the efficient synthesis of $1,1-\left[{ }^{18} \mathrm{~F}\right]$ difluorination of alkenes using non-carrier added $\left[{ }^{18} \mathrm{~F}\right]$-fluorine and fluoroalkenyl(4-methoxyphenyl)iodonium triflates. ${ }^{71}$ Such a method may be extremely useful for future labelling of fluoronucleosides, and other drugs containing a difluoro motif, because of its rapid reaction time (20 minutes), and compatibility with automated synthesis units. ${ }^{71}$ The alkene functionality also leaves room for a number of post-labelling modifications. ${ }^{71}$ 

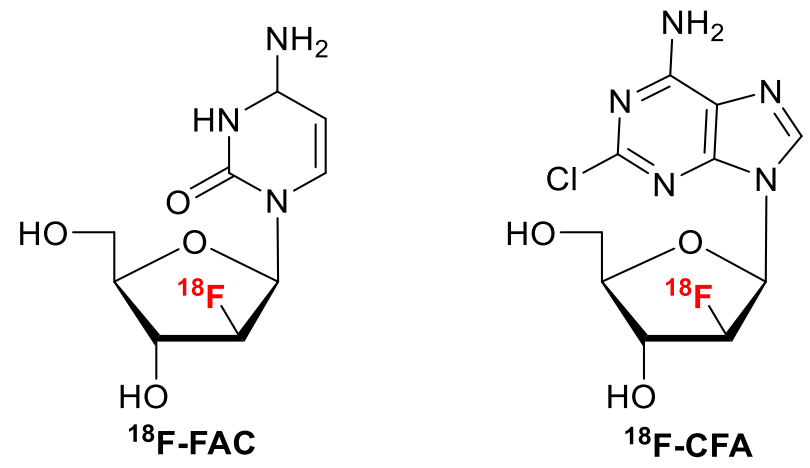

Figure 10. Examples of nucleoside-based PET radiotracers

Another important nucleoside-based PET imaging agent which has been reported recently is ${ }^{18} \mathrm{~F}$-clofarabine $\left({ }^{18} \mathrm{~F}\right.$-CFA; Figure 10$) .{ }^{65}$ The analogue is fluorinated with ${ }^{18} \mathrm{~F}$ at the $\mathrm{C} 2$ ' position retaining the original sugar modification necessary for RNR inhibition and anticancer activity. The ${ }^{18} \mathrm{~F}-\mathrm{CFA}$ was found to be a good probe for dCK activity in mice and humans, giving increased signal in tissues with increased dCK activity, such as cancer cells. Accurately monitoring dCK activity is of critical importance in determining efficacy of nucleoside prodrug anticancer agents. ${ }^{65}$

\subsubsection{2 ${ }^{68} \mathrm{Ga}$ based radiopharmaceuticals}

Recent advances in aza-crown ethers bifunctional chelators with the ability to bind gallium generated significant interest in ${ }^{68}$ Ga-based radiopharmaceuticals ${ }^{72-76}$ and radiotracers. ${ }^{76,77}$ As a metal salt, gallium has been reported to possess anti-proliferative properties in various cancer cells mainly attributed with its ability to mimic $\mathrm{Fe}^{3+} \cdot{ }^{78-80}$ Among bifunctional chelators, NODA-SA ${ }^{81}$ (1,4,7-triazacyclononane-1-succinic acid-4,7diacetic acid) and NODA-GA ${ }^{82}$ (1,4,7-triazacyclononane-1-glutamic acid-4,7-diacetic acid), which possess a NOTA (1,4,7-triazacyclononane-1,4,7-triacetic acid) complexation

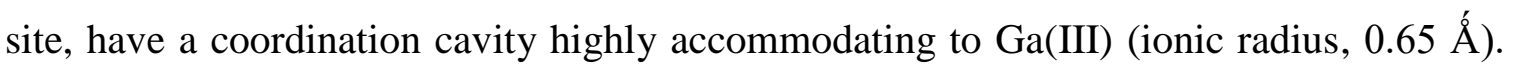
Also, anti-TF (Tissue Factor) antibody (ALT-836) conjugated to 4-isothiocyanatobenzyl- 
NOTA (SCN-Bn-NOTA) labelled with ${ }^{64} \mathrm{Cu}$ showed uptake in pancreatic cancer cells overexpressing TF and has been advanced to Phase I clinical trials as a combination therapy with gemcitabine.${ }^{83}$ Conjugation of SCN-Bn-NOTA with cyclic Arg-Gly-Asp -D-Tyr-Lys [c(RGDyK)] and chelated with ${ }^{68} \mathrm{Ga}$ gave a radiotracer for diagnosis of angiogenesis (Figure 11). ${ }^{77}$ These results encouraged us to investigate $4-\mathrm{N}$-alkylgemcitabine analogues conjugated with aza-crown ether chelators for ${ }^{68} \mathrm{Ga}$-labelling.

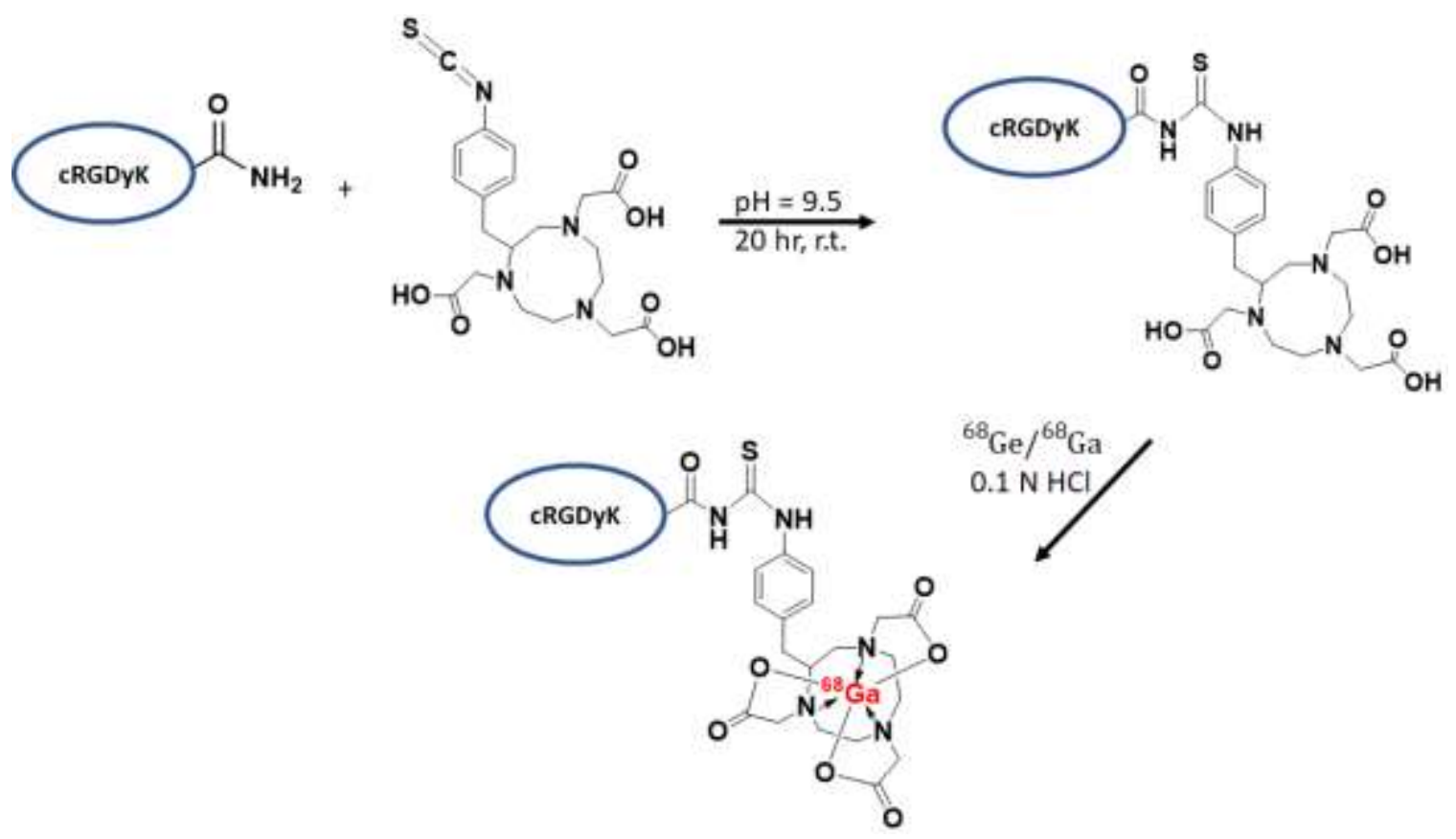

Figure 11. Example of c(RGDyK)-SCN-Bn-NOTA chelator with ${ }^{68}$ Ga-radiolabel ${ }^{77}$

\subsection{Radical induced DNA damage in radiotherapy}

Typical cellular activity in the body causes formation of reactive oxygen species (ROS), such as the hydroxyl radical $(\bullet \mathrm{OH})$, that either adds to DNA bases or abstracts an $\mathrm{H}$-atom from the sugar-phosphate backbone ${ }^{84,85}$ causing oxidative damage ${ }^{84}$ The damage can cause an increase in the rate of DNA mutations and uncontrolled cell proliferation, which have all been linked to cancer initiation and proliferation. ${ }^{86,87}$ In general, nucleobases are much more reactive to oxidation than the sugar-phosphate groups in 
nucleotides ${ }^{88}$ As a result of having the lowest ionization potential and redox potential, ${ }^{85,89-}$ 95 the guanine base (in 2'-deoxyguanosine) undergoes oxidation more readily than other bases or the sugar-phosphate backbone. ${ }^{96}$ Pulse radiolysis studies in neutral aqueous solutions at room temperature have shown the reaction of $\bullet \mathrm{OH}$ with guanine or with adenine eventually leads to the same guanyl or adenyl radical that is produced via direct one-electron oxidation of these bases followed by deprotonation. ${ }^{85,89,97,98}$ Therefore, understanding the mechanism of oxidative damage on dGuo and other nucleosides, then, is of high interest in the field of cancer research and treatment.

Most commonly, cancers are treated using either surgical, radiotherapeutic, chemotherapeutic methods, or combinations of the three methods. Unfortunately, surgery and chemotherapy can lack efficacy because of toxicity or lack of selectivity. ${ }^{99,100}$ Thus, the use of radiotherapy is becoming important, especially in combination with chemotherapy (chemoradiotherapy). ${ }^{100,101}$ Chemoradiotherapy involves the delivery of a sensitizer to the tumor followed by irradiation, which usually forms radicals which can interact with cellular DNA. ${ }^{99}$ The combination causes irreparable damage to the DNA inside cells, ultimately causing necrosis, apoptosis, or senescence. ${ }^{99,100}$

The downfall of chemoradiotherapy, is the hypoxic environment that is found in many tumor cells. The lack of $\mathrm{O}_{2}$ in these causes a large decrease in the efficacy of ionizing radiation because of decreased formation of the oxygen radicals that usually aid in initial DNA damage. ${ }^{99,102}$ Luckily, solvated electrons can be formed in as much quantity in hypoxic cells, as $\bullet \mathrm{OH}$ is formed in oxygenated cells..$^{99,103}$ The driving force of research in the area is the generation of radiosensitizers, like some modified nucleosides, which can incorporate onto DNA and have a high electron affinity. These sensitizers can undergo 
dissociative electron attachment via their reaction with radiation-produced electrons, which leads to radicals which can destroy cellular DNA. ${ }^{99}$

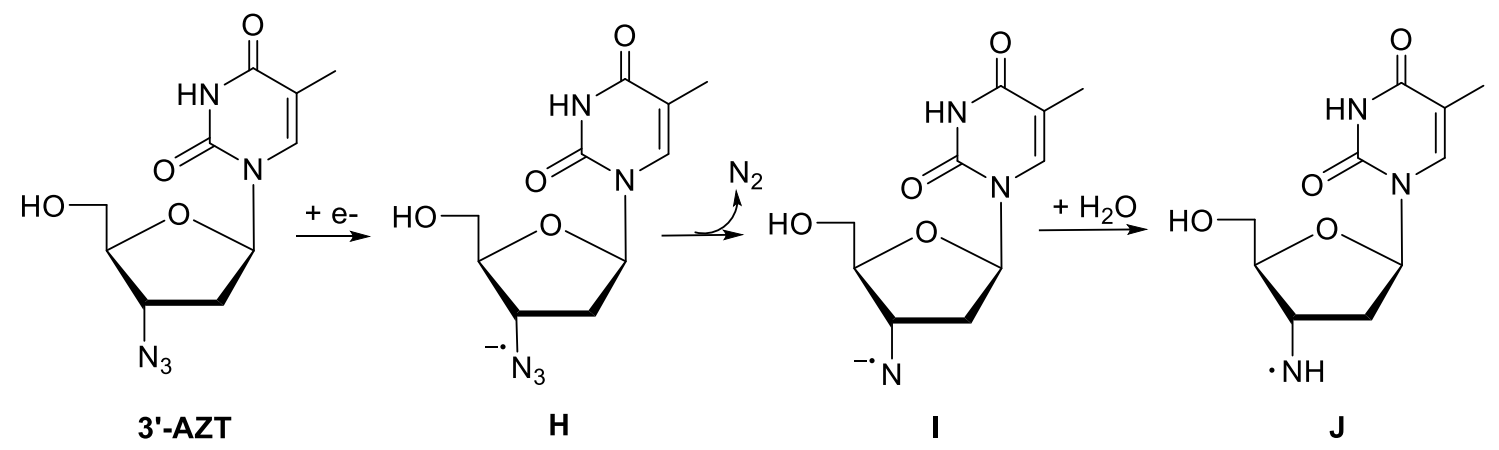

Figure 12. Proposed mechanism of aminyl radical formation in $3^{\prime}-\mathrm{AZT}^{104}$

One example of a drug which has chemoradiotherapeutic effects is 3'-AZT (3'-azido3'-deoxythymidine, Figure 12), which is used as an anti-viral in the treatment for HIV. It was found that HIV patients with cancer, taking 3'-AZT, responded better to treatment for solid tumors than cancer patients without HIV. It was discovered that these radiosensitizing effects are caused azido group present at the $3^{\prime}$ position of AZT that has high electron affinity and can capture an electron to form an unstable azide anion radical $\left(\mathrm{RN}_{3}{ }^{\circ}, \mathbf{H}\right)$ when irradiated with electrons. The species can then lose $\mathrm{N}_{2}$, leaving a nitrene anion radical $\left(\mathrm{RN}^{\bullet}, \mathbf{I}\right)$ which upon protonation becomes an aminyl radical $\left(\mathrm{RNH}^{\bullet}, \mathbf{J}\right)$. The formed aminyl radical is thought to be responsible for the DNA damaging effects of 3'-AZT in tumor cells. ${ }^{104}$

Previously, our lab in collaboration with the Sevilla-Adhikary lab at Oakland University has studied the formation of the elusive aminyl radical in both the sugar ${ }^{105}$ and base moiety ${ }^{106}$ of nucleosides. Recently, two classes of azido modified pyrimidine analogues were studied. One class contains an azidomethyl group at C5 which includes: 5azidomethyl-2'-deoxyuridine (AmdU) and cytidine (AmdC, Figure 13, Panel A). The azido 
group in AmdU was found to undergo dissociative electron attachment resulting in a nitrene anion radical, which undergoes rapid protonation to form a $\pi$-aminyl radical. Subsequent abstraction of a proton from a nearby AmdU precursor molecule was then found to form an $\alpha$-azidoalkyl radical, that underwent unimolecular $\beta-\mathrm{N}_{2}$ elimination to form the more stable $\sigma$-iminyl radical. A similar mechanism and $\sigma$-iminyl radical formation was seen in AmdC. AmdU was found to have radiosensitizing properties elective for treatment of EMT6 breast tumor models. The second class possesses an azidobinyl group at C5 position that includes: 5-(1-azidovinyl)-2'-deoxyuridine (AmvU) and cytidine (AmvC, Figure 13, Panel B). These analogues were found to form a $\pi$-aminyl radical that easily tautomerized to the stable $\sigma$-iminyl radical. ${ }^{106}$ 


\section{Panel A}<smiles>[R][Al]n1cc(CNC(C)C)c(=O)[nH]c1=O</smiles>

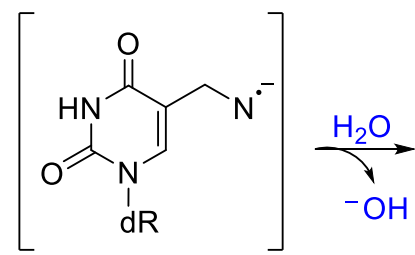

AmdU

Nitrene anion radical<smiles>[R]On1cc(C(O)N=O)c(=O)[nH]c1=O</smiles>

$\mathrm{U}-5-\mathrm{CH}_{2}-\mathrm{NH}^{\cdot}(\pi)$<smiles>[R][Tl]n1cc(C([1H])[AlH2])c(=O)[nH]c1=O</smiles><smiles>C/N=C\C1=CC(=O)C(=O)NC1=O</smiles><smiles>[Y8]Cc1cn([Tl])c(=O)[nH]c1=O</smiles><smiles>[R][R]n1cc(CN)c(=O)[nH]c1=O</smiles>

$\mathrm{U}-5-\mathrm{CH}^{\cdot}-\mathrm{N}_{3}$

$\mathrm{U}-5-\mathrm{CH}_{2}-\mathrm{NH}_{2}$

\section{Panel B}

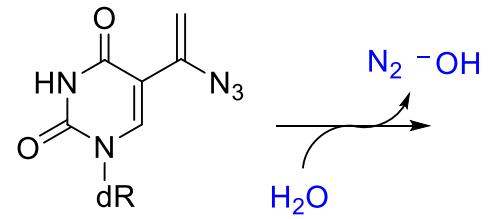

AvdU<smiles></smiles>

$\mathrm{U}-5-\mathrm{C}\left(\mathrm{NH}^{-}\right)=\mathrm{CH}_{2}$

$(\pi)$<smiles>C=N/C(C)=C(/C)c1cn([Ga]C)c(=O)[nH]c1=O</smiles>

$\mathrm{U}-5-\mathrm{C}\left(\mathrm{CH}_{3}\right)=\mathrm{N}^{\cdot}$

$(\sigma)$

Proposed mechanism for the formation of $\pi$-aminyl radical from AmdU, followed by its biomolecular conversion to a $\sigma$-iminyl radical (Panel A). Tautomerization of $\pi$-aminyl radical from AvdU to $\sigma$-iminyl radical (Panel $\mathrm{B}$ ).

Figure 13. Proposed mechanism for formation of $\pi$-aminyl radical and $\sigma$-iminyl radical from AmdU and AvdU. ${ }^{106}$

\subsubsection{Radical formation on the guanine base caused by DNA oxidation}

Many research groups have been interested in understanding the chemistry of purine nucleosides oxidation; including mechanism, intermediates and products under different oxidative conditions. The radical chemistry of oxidative damage of purines is studied either to understand its reaction with $\bullet \mathrm{OH}$, or via one-electron oxidation. ${ }^{89}$ Experimentally, oneelectron oxidation can be studied using a strong oxidant such as $\mathrm{SO}_{4} \cdot{ }^{-85,89,97}$ 
The most common structure studied of the reaction of guanine with $\bullet \mathrm{OH}$ is 8 -oxo-7,8dihydro-2'-deoxyguanosine (8-oxodGuo). The 8-oxodGuo is only one of the many products of guanine oxidation and is used as a biomarker for oxidative stress. The 8oxodGuo is formed by reaction with hydroxyl radical, singlet oxygen or one-electron oxidants. ${ }^{96,107-109}$ Figure 14 shows the mechanism of formation of the main 6,8-diketo tautomer of 8 -oxodGuo made via reaction of guanine by $\bullet \mathrm{OH}$.

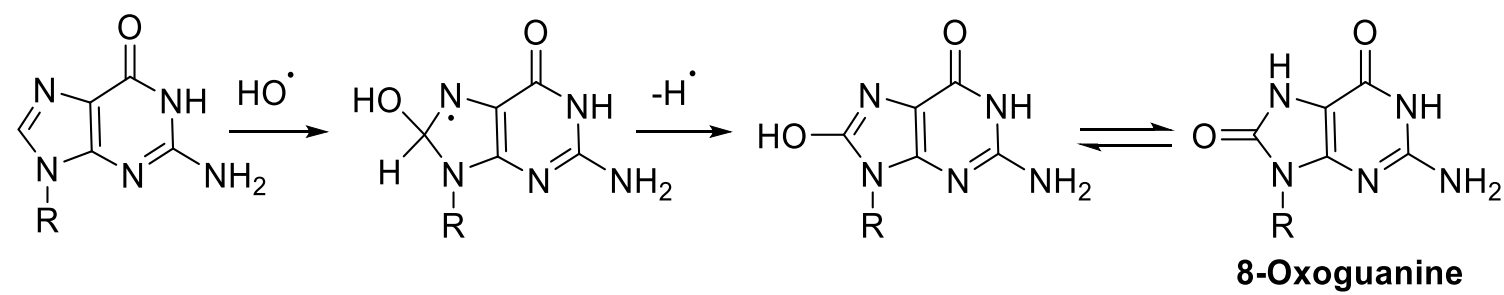

Figure 14. Formation of 8 -oxoguanine by reaction with $\bullet \mathrm{OH}$

The $\cdot \mathrm{OH}$ has a high reduction potential $(2.3 \mathrm{~V}$ at $\mathrm{pH} 7) ;^{85,91,110}$ however, it does not cause direct one-electron oxidation. ${ }^{91,110}$ Theoretical studies on one-electron oxidation by -OH have shown that solvation of the $-\mathrm{OH}$, produced after electron transfer to $\bullet \mathrm{OH}$, accounts for most of the reduction potential of $\bullet \mathrm{OH}$. As a result, the initial electron transfer step that is involved in the direct one-electron oxidation by $\bullet \mathrm{OH}$ becomes slow. ${ }^{110,111}$ Thus, reaction of $\bullet \mathrm{OH}$ with purine nucleosides occurs by its direct addition to the double bonds of the guanine ring. Thus, for many years, it was thought the 8-OxodGuo was the main product of guanine oxidation. However, it is now understood that it is just one of many intermediates, and undergoes further oxidation to form imidazolone and oxazolone products. $^{109,112,113}$

\subsubsection{Studies on the formation of guanyl and adenyl aminyl radical}

The accepted mechanism of the reaction of $\bullet \mathrm{OH}$ with the guanine base is the addition of $\bullet \mathrm{OH}$ at $\mathrm{C} 4$ leaving a radical at $\mathrm{C} 5$ (i.e. L, Figure 15), which undergoes dehydration to 
form neutral radical $\mathbf{M} \cdot{ }^{113,114}$ The radical $\mathbf{M}$ forms from deprotonation of the guanine cation radical, $\mathrm{G}(\mathrm{N} 1-\mathrm{H}))^{\bullet}$. Guanine cation radical $\left(\mathrm{G}^{\bullet^{+}}\right)$is known to undergo competitive reactions: (a) deprotonation leading to $\mathrm{G}(\mathrm{N} 1-\mathrm{H}) \bullet$ formation (i.e., phototropic equilibria of $\mathrm{G}^{\bullet+}$ ), and (b) nucleophilic addition of water at $\mathrm{C} 8$ leading to $\mathrm{G} \bullet-\mathrm{OH}$ followed by oneelectron oxidation to form 8-oxo-guanine..$^{90-95,97,109}$ Pathway (a) was thought to be the major pathway ( $65 \%)$, while pathway (b) was thought to be the minor pathway $(17 \%) .{ }^{114,115}$ In 2009, studies by Chatgilialoglu and co-workers proposed that the main pathway of hydroxyl radical oxidation of guanine is not the addition at the $\mathrm{C} 4$ position, but rather a hydrogen abstraction from the exocyclic $\mathrm{NH}_{2}$ moeity to form an aminyl radical [G(N2-H)•; e.g., radical N]. ${ }^{115}$ The N-centered neutral aminyl radical formation was proposed because of the formation of a broad peak around $610 \mathrm{~nm}$ obtained from pulse radiolysis with optical absorption experiments of 8-bromoguanine $\mathbf{O} .{ }^{115}$ Under oneelectron oxidation conditions, using $\mathrm{SO}_{4}{ }^{--}$as the oxidant, it was found that guanine formed its radical cation followed by loss of hydrogen to form radical $\mathbf{N}$, which is a tautomer of M, giving the same optical absorption at $610 \mathrm{~nm} \cdot{ }^{89,115}$

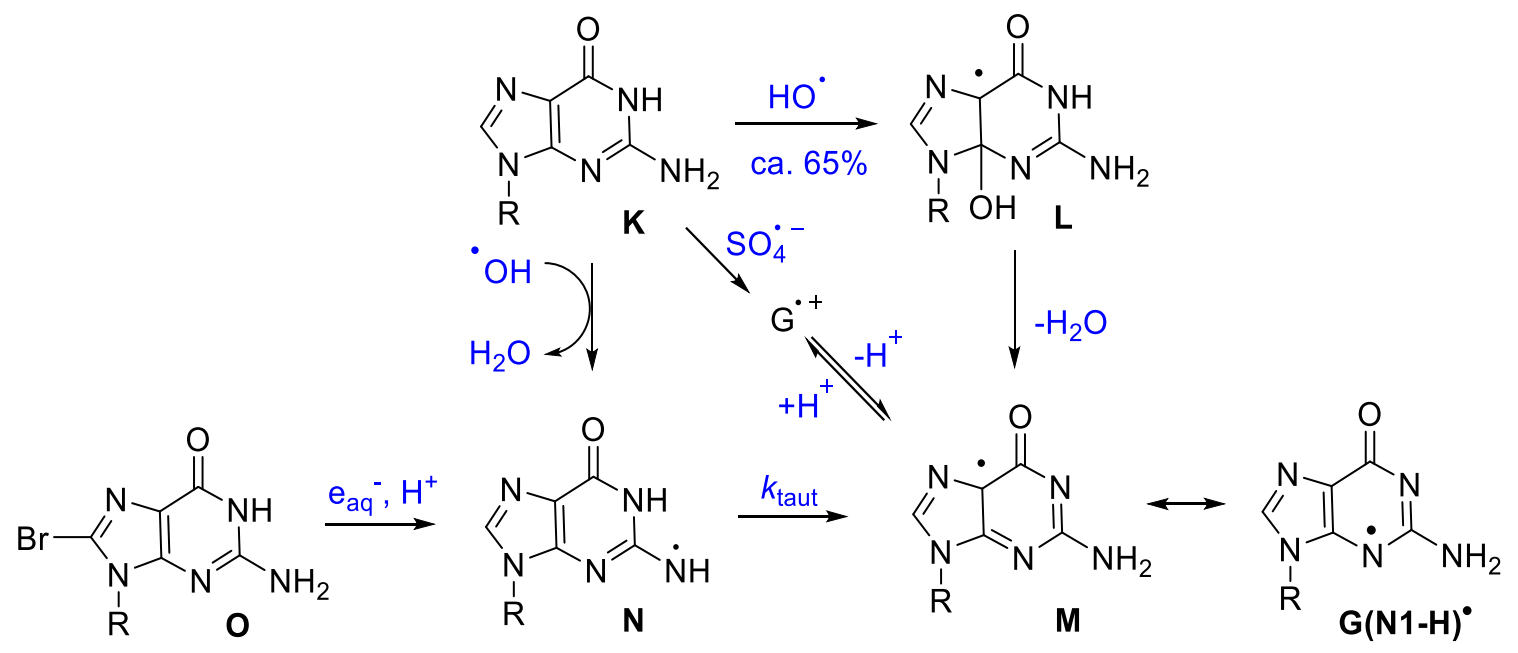

Figure 15. Chatgilialoglu's proposed $\bullet \mathrm{OH}$ oxidation mechanism of guanine base $\mathrm{e}^{115}$ 
Similar to guanine, adenine usually forms $\bullet \mathrm{OH}$ adduct at the $\mathrm{C} 4$ and $\mathrm{C} 8$ positions, with the former being oxidizing and the latter being primarily reducing. The $\mathrm{C} 4-\mathrm{OH}$ adducts of adenine can further interact with the environment to make a variety of products, most of which are unknown. ${ }^{84,116}$ As with guanine, the formation of 8-hydroxyadenine is quite common especially in the presence of oxygen. ${ }^{84,117}$ Another common byproduct formed both from reaction with $\bullet \mathrm{OH}$ and under one-electron oxidation conditions, with a strong oxidant such as $\mathrm{SO}_{4}{ }^{-}$, is an $\mathrm{N} 6$ centered aminyl radical that is the deprotonated adenine cation radical. Although, under one-electron oxidation conditions, a cation radical is formed first, which upon deprotonation converts to the aminyl radical. ${ }^{89}$

Many attempts have been made, by Chatgilialoglu ${ }^{115,118}$ and others to identify the aminyl radical formed from guanine, and N6 position of adenosine, using photochemical precursors. However, characterization of these radicals employing Electron Paramagnetic Resonance (EPR) or Electron Spin Resonance (ESR) spectroscopy were never achieved. One example is Dr. Wagner's group, who demonstrated the formation of $\mathrm{N}^{6}$ aminyl radicals by photolysis of 6- $N$-phenylhydrazones of 2'-deoxyadenosine in the presence of $\mathrm{H}$-donors, like glutathione (Figure 16,P). ${ }^{119}$ Evidence from high-performance liquid chromatography (HPLC), mass spectrometry (MS), and NMR indicated the formation of the aminyl radical formation because of the detection of 2'-deoxyadenosine, and benzaldehyde byproduct. ${ }^{119}$ More recently, Dr. Greenberg's group identified the aminyl radical by photolysis of the hydrazine modifications of the 2- $\mathrm{N}$-positon of guanine $(\mathbf{Q})$ and 6- $\mathrm{N}$-positon of adenosine (R). Also, the Greenberg group showed the compatibility of these hydrazine analogues for solid-phase oligonucleotide synthesis, and thus, their utility for studying the aminyl radical's reactivity in nucleic acids. ${ }^{120}$ They determined formation of the adenyl aminyl 
radical by identification of 2'-deoxyadenosine and 8-diphenylamide-2'deoxyadenosine products. The guanyl aminyl radical was identified by formation of 2'-deoxyguanosine and corresponding 8-dipheynyl substituted byproduct. Interestingly, the guanyl aminyl radical was more thermodynamically stable and reacted less than the adenyl aminyl radical with $\mathrm{PhN} \cdot$ radical. ${ }^{120}$ Although these groups both studied the formation of the aminyl radical in a variety of ways, there was no conclusive EPR results which could unequivocally characterize the aminyl radical. ${ }^{119,120}$
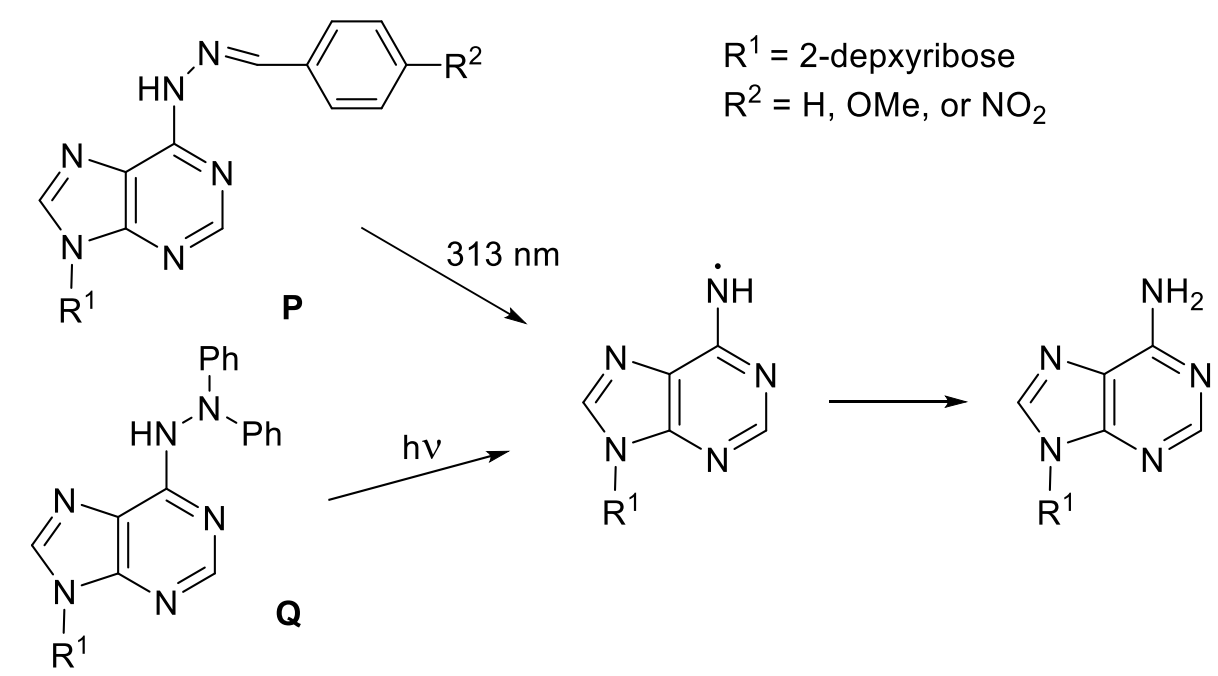<smiles>[R]n1cnc2c(=O)[nH]c(NN(c3ccccc3)c3ccccc3)nc21</smiles><smiles>[R]n1cnc2c(=O)[nH]c(N)nc21</smiles>

Figure 16. Formation of aminyl radical from hydrazines ${ }^{120}$ and hydrazones ${ }^{119}$

\subsubsection{Radical formation in DNA oxidation of pyrimidine bases}

Pyrimidine bases, thymidine and cytidine in DNA, also interact with ROS in the cell and form several reactive byproducts. ${ }^{84} \mathrm{~A}$ common reaction of the $\bullet \mathrm{OH}$ with pyrimidines involves addition to the C5-C6 double bond or by abstraction of $\mathrm{H}$ in methyl group of thymidine. A variety of different byproducts form depending on the redox properties, with 
$\mathrm{C} 5-\mathrm{OH}$ adducts being reducing and $\mathrm{C6}-\mathrm{OH}$ adducts being oxidizing, and environment of each new radical that is made. ${ }^{84,121}$ The most common products made from the oxidation of cytidine and thymine under anoxic conditions is cytosine glycol and thymine glycol (Figure 17). ${ }^{84}$ The H-atom abstraction from methyl group of thymidine causes formation of an allyl radical which ultimately leads to the formation of 5-hydroxymethyluracil, in the absence of oxygen. ${ }^{84,122}$ These byproducts can further interact with their environment and other radicals to form additional species. ${ }^{84}$<smiles>[R]N1C(=O)N=C(N)C(O)(O)C1([2H])O</smiles>

cytosine glycol<smiles>[R]N1C(=O)NC(=O)C(C)(O)C1(O)O</smiles>

thymine glycol<smiles>[R]n1c([2H])c(CO)c(=O)[nH]c1=O</smiles>

5-hydroxymethyluracil

Figure 17. Common byproducts of pyrimidine oxidation under anoxic conditions

Under one electron oxidation conditions, thymidine forms a cation radical, much like purine bases, which quickly undergoes addition of water to form an $\mathrm{OH}$ adduct or deprotonates. ${ }^{110,123}$ Usually, the $\bullet \mathrm{OH}$ adds onto the $\mathrm{C} 5$ position of thymidine, leaving a $\mathrm{C} 6$ radical, which then tautomerizes to the $\mathrm{C} 6 \mathrm{OH}$ adduct with radical site at $\mathrm{C} 5$ (Figure 18). ${ }^{110}$

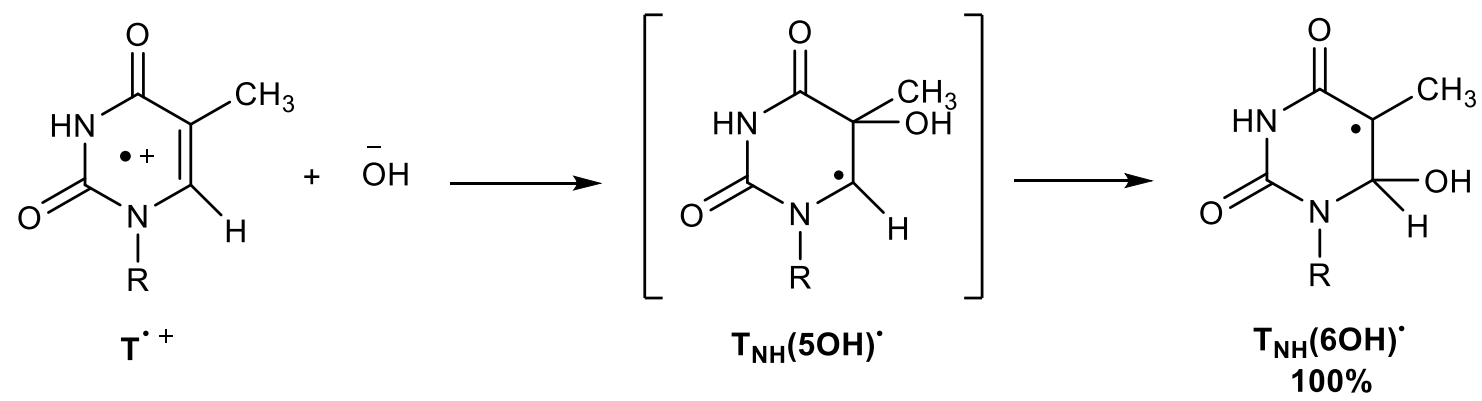

Figure 18. Addition of hydroxy anion to one-electron oxidized thymidine ${ }^{110}$ 
Greenberg's group found that abstraction of hydrogen from C5 methyl group of thymine base in poly(dA-T) DNA sequences occurs, not from deprotonation or hydration of the thymidine cation radical, but rather from interaction with an adenosine N6 aminyl radical. ${ }^{124}$ Since purines have lower reduction potentials than pyrimidines, they form radical cations more readily than pyrimidines. Thus, they are the main source of radical induced damage on DNA. The N6 aminyl radical of adenosine can cause abstraction of a C5 methyl hydrogen of 5'- flanking thymidines, leading to the allyl radical formation and this radical eventually produces the 5-formyl-2'deoxyuridine. ${ }^{124}$

One electron-oxidation of cytidine, using $\mathrm{SO}_{4}{ }^{--}$, was found to form a neutral aminyl radical at the N4 position by a radical cation intermediate. ${ }^{123}$ In 2015 , Adhikary-Sevilla et al. showed that the cytidine cation radical preferentially deprotonated to form an aminyl radical syn to the carbonyl moiety. The syn-aminyl radical then tautomerizes to the iminyl $\sigma$-radical (Figure 19). The mechanism was found to prevail over addition of water to the C5-C6 bond that is seen in thymidine. ${ }^{125}$ Theoretical calculations also illuminated the ability of the iminyl radical to form in double-stranded DNA through radiation induced ionization-deprotection processes as proposed employing pulse radiolysis studies. ${ }^{125,126}$

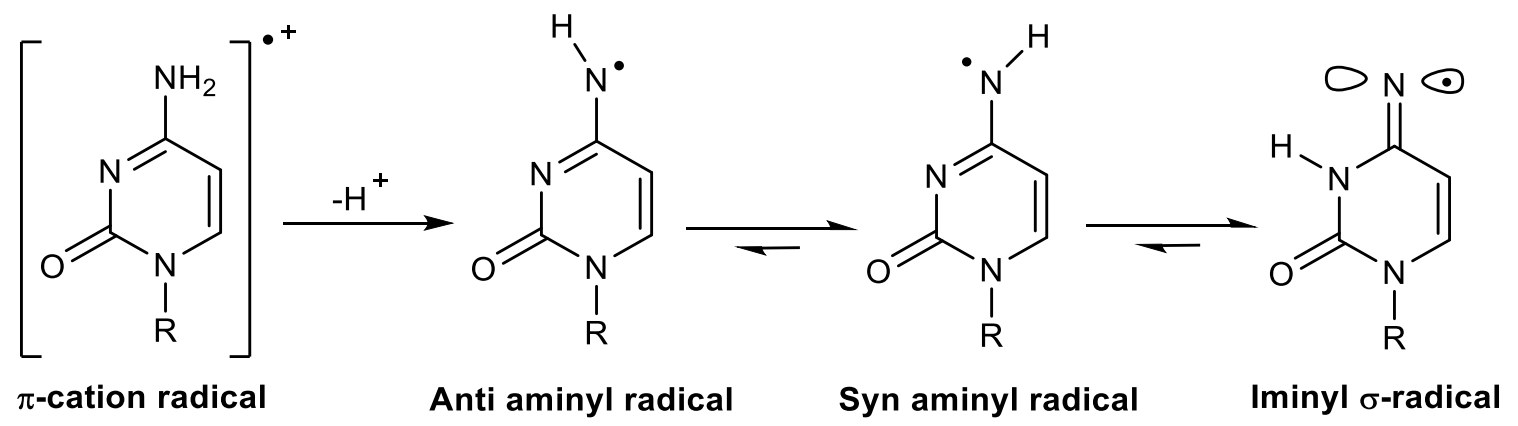

Figure 19. Cytidine syn aminyl radical tautomerization to iminyl radical ${ }^{125}$ 


\subsection{Azido modified nucleosides}

Many azido-substituted nucleosides have been synthesized as anti-viral or anti-cancer agents, and a prodrugs to increase efficacy of NA drugs. ${ }^{127-131}$ Perhaps the most important example is 3'-azido-3'-deoxythymine (AZT) which was synthesized in 1964 as a possible anti-cancer agent, yet in 1987 was approved in record time as an anti-viral treatment for acquired immune deficiency syndrome (AIDS). Although AZT's mode of action was driven by the azido modification on the sugar moiety, other nucleoside analogues have been synthesized with azido modification in the base. ${ }^{132,133}$

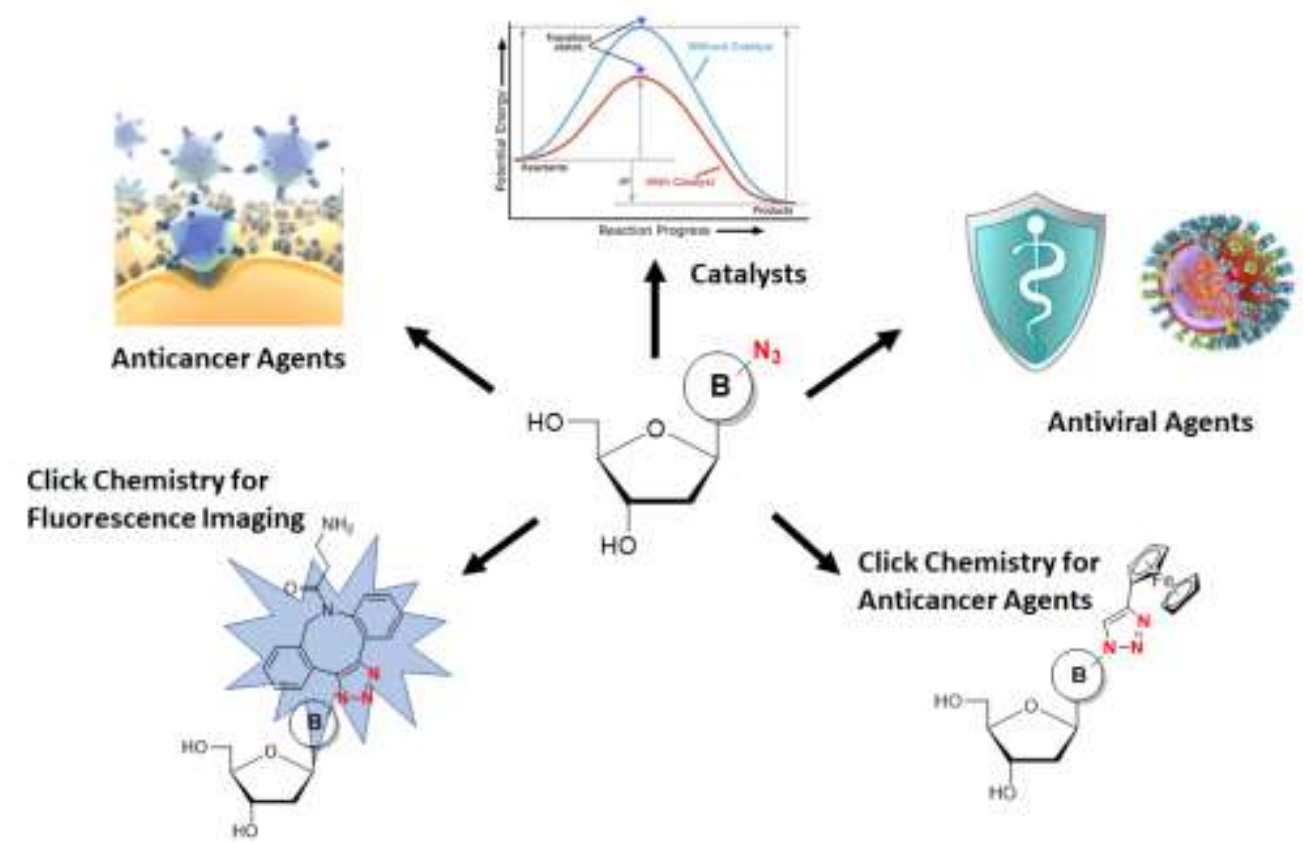

Figure 20. Common application of azido-modified nucleoside analogues

Both azido-substituted purines and pyrimidine nucleosides have been studied for their use in several areas of chemistry (Figure 20). For biological activity of certain azido modified analogues, it is essential that the azide is in its linear form and can interact with key enzymes. For example, the 6-azido modified purine prodrugs of 9-( $\beta$-Darabinofuranosyl)adenine (ara-A, Vidarabine) ${ }^{128}$ or 4-azido modified pyrimidine prodrug 
of 1 -( $\left(\beta\right.$-D-arabinofuranosyl)cytosine $(\operatorname{ara}-\mathrm{C})^{130}$ whose efficacy requires that azide be available for reduction to amine by enzymatic action. Other azido nucleoside analogues, such as 2, 6, or 8-azido modified purines, and 4- or 5- azido modified pyrimidines are synthesized to use for click-reactions, where the triazole click products can be used for fluorescence imaging of cancer cells ${ }^{134,135}$, as adenosine receptor antagonists, ${ }^{136}$ inhibitors of key enzymes for treatment of the tuberculosis bacterium, ${ }^{137}$ or anti-viral agents. ${ }^{138,139}$ Azide group in azido-modified nucleosides can often cyclize with nitrogen atoms present in the pyrimidine or purine ring to form tetrazole tautomers. The formation of the tetrazole often results in lack of prodrug activity, or low yield during click reactions because the stability of the cyclic tetrazole derivative often affects reactivity. ${ }^{130,140}$

\subsubsection{Synthesis and properties of azido modified nucleosides}

\subsubsection{Synthesis of azido modified purine nucleosides}

\subsection{2-Azidopurine nucleosides}

Synthesis of 2-azidopurine nucleoside analogues has been undertaken in order to study their anti-viral properties, ${ }^{141}$ or their use for determining protein-DNA/RNA interactions. ${ }^{142,143}$ The analogues have been synthesized either from adenosine or guanosine precursors. For example, oligonucleotides containing 2-azido-2'-deoxyinosine (i.e., 3, Scheme 1) has been synthesized from their protected 2-fluoro-2'-deoxyinosine precursor (i.e., 1). ${ }^{143}$ The azido group is highly reactive during oligonucleotide synthesis undergoing Staudinger reduction with phosphoramidite reagent. Thus, incorporation of azido-modified nucleosides onto DNA/RNA has not been fully developed. ${ }^{144,145}$ Preparation of 3 began by protection of the 6 position of guanosine using 2-(4nitrophenyl)ethyl (NPE), followed by diazotization/fluorination. Then, the 2-fluoro-2'- 
deoxyinosine 2-cyanoethyl phosphoramidite precursor 1 was synthesized for solid phase synthesis. After solid-phase elongation and treatment with 1,8-diazabicyclo[5.4.0]undec7-ene (DBU) a ODN fragment 2 was prepared. Finally, reaction with lithium azide $\left(\mathrm{LiN}_{3}\right)$ in dry dimethylformamide gave desired azido analogue 3. Subsequent deprotection with ammonia lead to removal from solid support and formation of oligonucleotides containing 2-azido-2'-deoxyinosine. ${ }^{143}$
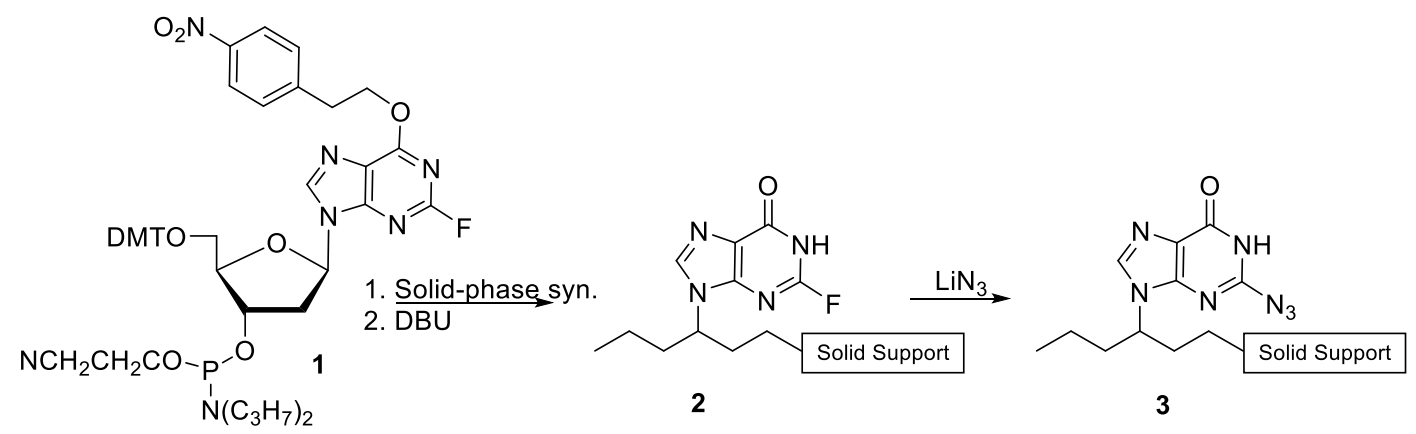

Scheme 1. Synthesis of 2-azido-2'-deoxyinosine from 2-fluoro-2'-deoxyinosine

The 2-azidopurine analogues have also been synthesized from guanosine and 2'deoxyguanosine by diazotization of the $\mathrm{C} 2$ amino-group followed by replacement with azide. Guanosine derivatives were first converted into their protected 6-chloropurine analogues or 6-O-mesitylated analogues to improve solubility in organic solvents. ${ }^{142,146}$ The method was first reported by Higashiya et al. for the synthesis of 2-azido-2'deoxyadenosine (Scheme 2). Diazotization of the amino group in 4 with isoamyl nitrite, followed by azidation with azido-trimethyl silyl (TMS-N 3 ) gave $5 .{ }^{146}$ Similarly, the 6-Omesitylated analogue 7 was treated with $t \mathrm{BuONO} / \mathrm{TMS}^{-N_{3}}$ gave 2 -azido analogue $8{ }^{142}$ In either case, 6-modified derivatives were converted to 6- $\mathrm{NH}_{2}$ (adenosine; e.g., 6 and 9) derivatives using concentrated ammonia reagents. ${ }^{142,146}$ 

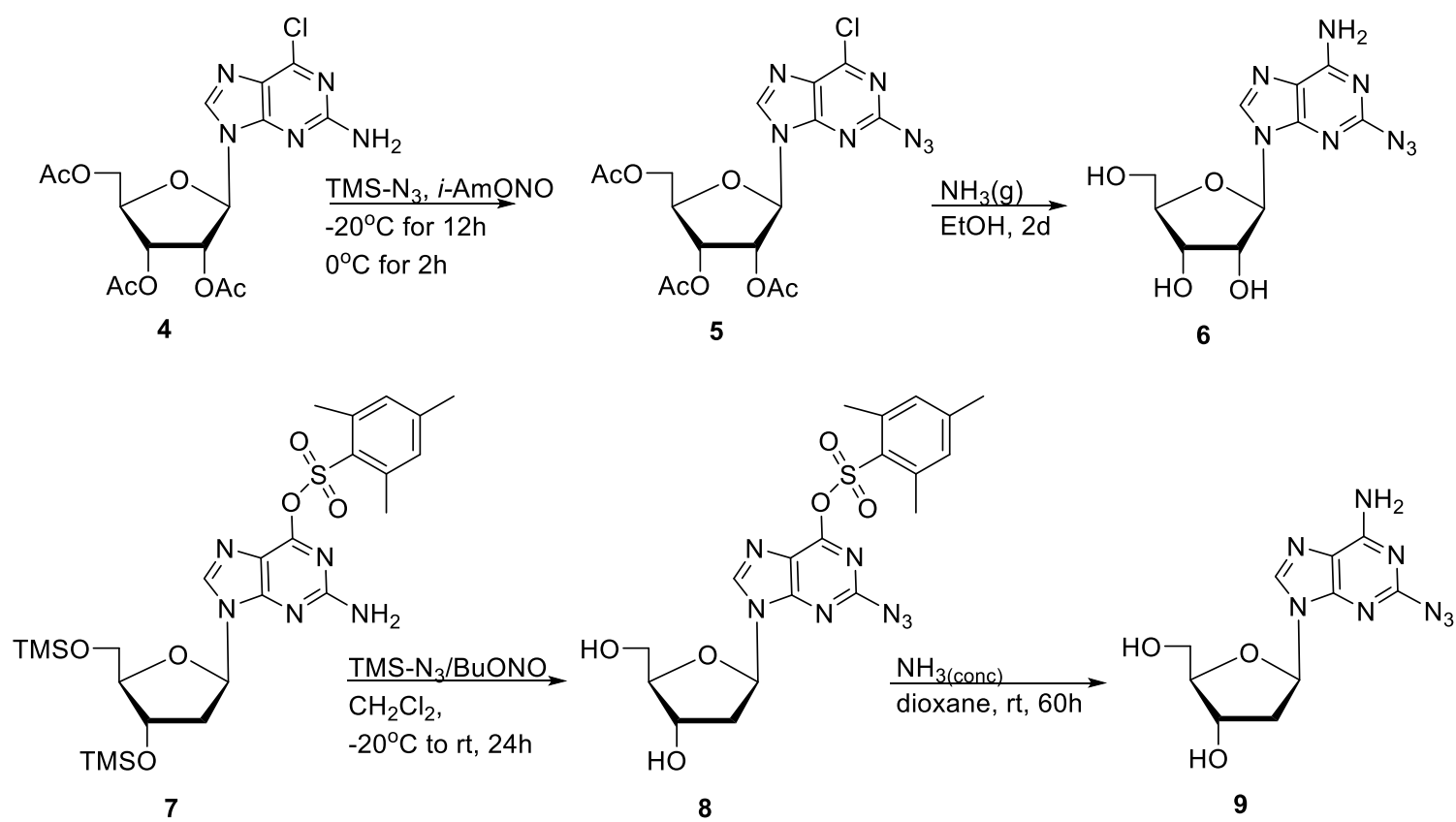

Scheme 2. Approaches towards synthesis of 2-azidoadenosine and 2-azido-2'deoxyadenosine

The 2-azidoadenosines have also been synthesized from their 2-chloroadenosine precursors (i.e. 10, Scheme 3). Thus, compound 10 was converted to 2-hydrazino 11, followed by treatment with $\mathrm{NaONO} / \mathrm{AcOH}$ in acidic medium to yield 12. The tautomerization to tetrazole 12a was documented by two sets of signals present on NMR, where tetrazole peaks are more deshielded. Table 1 shows NMR peaks for azido/tetrazole tautomers of 2-azidoadenosine analogues. ${ }^{141}$

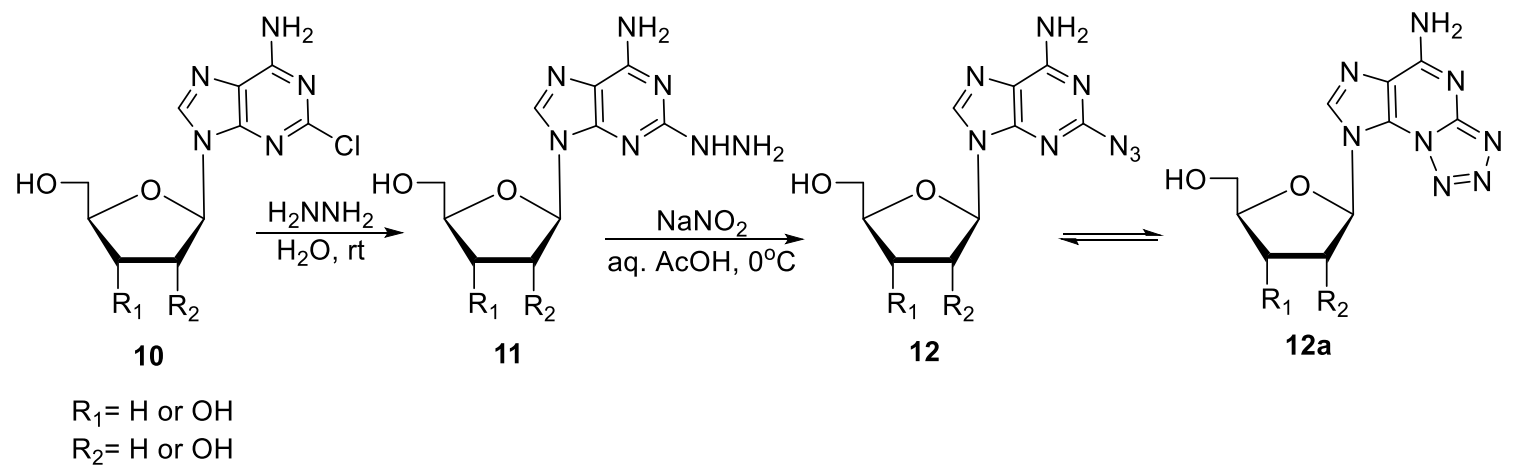

Scheme 3. Synthesis of 2-azidopurine nucleoside and its tetrazole tautomer 
Table 1. ${ }^{1} \mathrm{H}$ NMR Chemical Shifts of Azido/Tetrazole (A/T) Tautomers of 2-azidoadenine nucleosides

\begin{tabular}{|c|c|c|c|c|c|c|}
\hline $\mathrm{R}_{1}$ & \multicolumn{2}{c|}{$\mathrm{OH}$} & \multicolumn{2}{c|}{$\mathrm{OH}$} & \multicolumn{2}{c|}{$\mathrm{H}$} \\
\hline $\mathrm{R}_{2}$ & \multicolumn{2}{c|}{$\mathrm{OH}$} & \multicolumn{2}{c|}{$\mathrm{H}$} & \multicolumn{2}{c|}{$\mathrm{H}$} \\
\hline Azido form & $8 \mathrm{H}$, & $6 \mathrm{NH}_{2}$, & $8 \mathrm{H}$, & $6 \mathrm{NH}_{2}$, & $8 \mathrm{H}, 8.29$ & 7.60 \\
& 8.28 & 7.67 & 8.25 & 7.60 & & $6 \mathrm{NH}_{2}$, \\
\hline Tetrazole form & $8 \mathrm{H}$, & $6 \mathrm{NH}_{2}$, & $8 \mathrm{H}$, & $6 \mathrm{NH}_{2}$, & $8 \mathrm{H}, 8.59$ & $6 \mathrm{NH}_{2}, 9.4$ \\
& 8.56 & 9.46 & 8.53 & 9.40 & & \\
\hline$\Delta \delta(\mathrm{ppm})$ & 0.28 & 1.79 & 0.28 & 1.80 & 0.30 & 1.80 \\
\hline Ratio A/T & \multicolumn{2}{|c|}{$67: 33$} & \multicolumn{2}{c|}{$65: 35$} & \multicolumn{2}{c|}{$67: 33$} \\
\hline
\end{tabular}

Alternatively, synthesis of 2-azidoadenosine analogues $\mathbf{1 5}$ has also been achieved from $\mathrm{N}^{6}$-methyl-2-iodoadenosine analogue $\mathbf{1 4}$ by reaction with $\mathrm{CuSO}_{4} /$ sodium ascorbate catalyst in the presence of $\mathrm{L}$-proline, $\mathrm{Na}_{2} \mathrm{CO}_{3}$, and $\mathrm{NaN}_{3}$ in a mixture of water/t-butanol (Scheme 4). Compound 15 showed high affinity for $A_{3}$ adenosine receptor, with some of its click products being full antagonists. The $\mathrm{N}^{6}$-methyl-2-azidoadenosine derivative 15 had to be isolated to proceed with click-reactions. Tautomerization to the tetrazole derivative $\mathbf{1 5 a}$ was seen in about $17 \%$ for $\mathrm{N}^{6}$-methyl-2-azidoadenosine $15 .{ }^{136}$

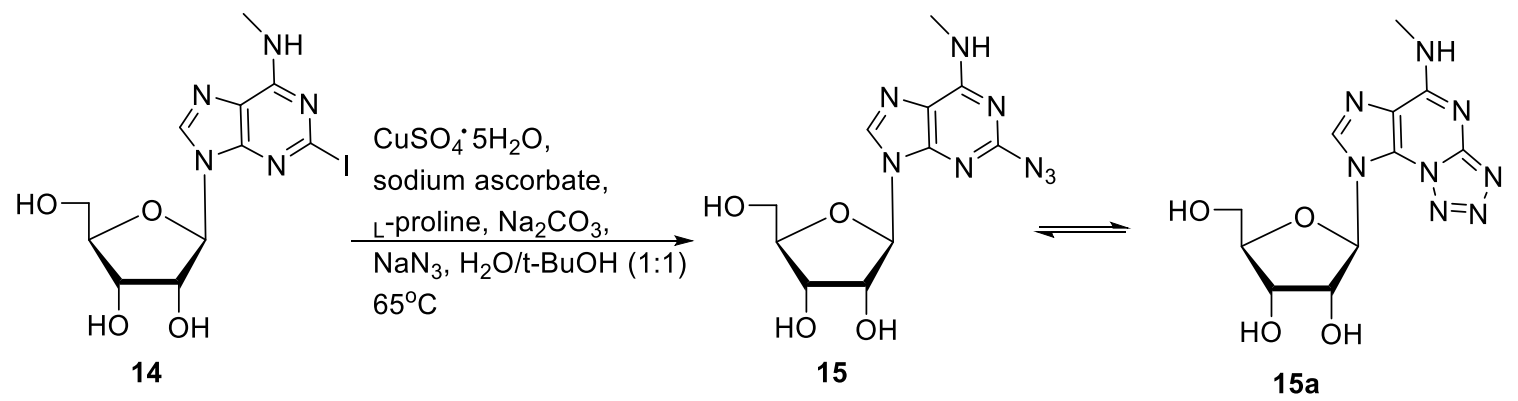

Scheme 4. Synthesis of 2-azido- $N^{6}$-methyladenosine from 2-iodo- $N^{6}$-methyladenosine and its equilibrium with its corresponding tetrazole

\subsection{8-Azidopurine nucleosides}

The 8-azidoadenosine analogues have been explored for their use in photochemical cross-linking that determine protein-nucleic acid interactions. ${ }^{143}$ Synthesis of 8azidoadenosine derivatives has been achieved from the reaction of 8-bromoadenosine with 
sodium azide. ${ }^{147}$ Incorporation of azido-modified nucleosides onto DNA/RNA is an area has not been fully developed because of the high reactivity of the azide moiety during solidphase synthesis, although post-synthetic modifications have been achieved. ${ }^{144,145}$

To synthesize oligodeoxynucleotides (ODN) containing 8-azido-2'-deoxyadenosine, substitution with azide was explored after preparation of the phosphoramidite derivative 16 (Scheme 5). The $N^{6}$-position of 8-bromo-2'-deoxyadenosine was protected with base labile dimethylformamidine, which can be introduced without the need to protect sugar hydroxyls. Then, regioselective protection of the 5'-hydroxyl with dimethoxytrityl (DMT), and subsequent reaction with $\mathrm{N}, \mathrm{N}$-diisopropyl-O-2-cyanoethylchlorophosphine formed phosphoramidite analogue 16. Displacement of bromide with azide employing lithium azide in DMF at $70^{\circ} \mathrm{C}$ yielded 8-azido-2'-deoxyadenosine derivative $17 .{ }^{143}$

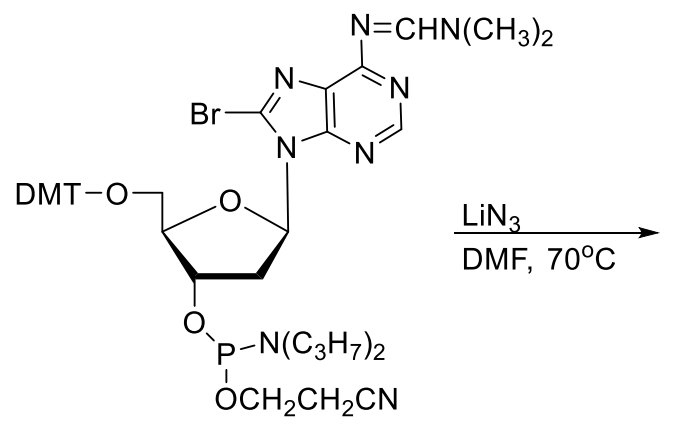

16

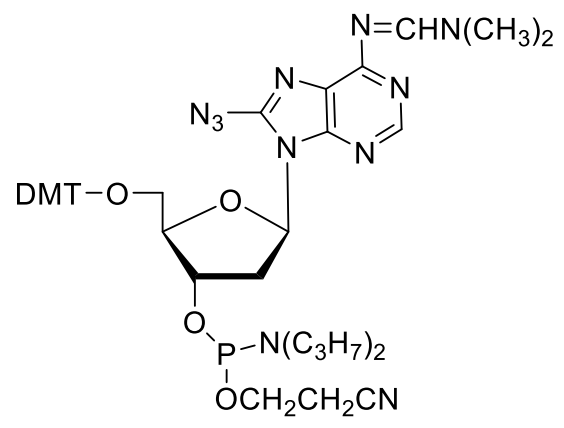

17

Scheme 5. Synthesis of 8-azidoadenosine monomer for the synthesis of ODN via solidphase synthesis

\subsection{6-Azidopurine nucleosides}

Synthesis of 6-azidopurine analogues can be accomplished from their respective inosine derivatives for use as Ara-A prodrugs, ${ }^{128}$ or as anti-viral agents. ${ }^{131}$ These methods have been developed to improve upon previous protocols which included chlorination of the 6-position of inosine followed by replacement with azide, ${ }^{140}$ which gave low to moderate yields as a consequence of decomposition. ${ }^{128,148}$ Using $O^{6}$-(benzotriazole-1- 
yl)inosine ${ }^{149} \mathbf{1 9}$ as a precursor, several 6-azidopurine analogues of type 20 were synthesized either by displacement of $O$-benzotriazole by sodium azide or by reaction with polymer supported azide (Scheme 6). The use of polymer supported azide was especially useful in synthesis of deprotected 6-azidopurine analogues due to its solubility in aqueous solvent. Table 2 shows reaction conditions and yield for several 6-azidopurine nucleoside analogues. ${ }^{140}$

$\mathrm{R}_{1}=\mathrm{H}$, TBDMS, or Ac

$\mathrm{R}_{2}=\mathrm{H}, \mathrm{OH}$, OTBDMS, or OAC

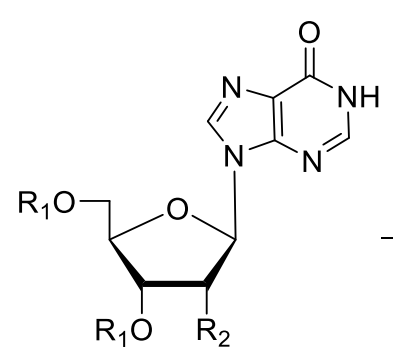

18

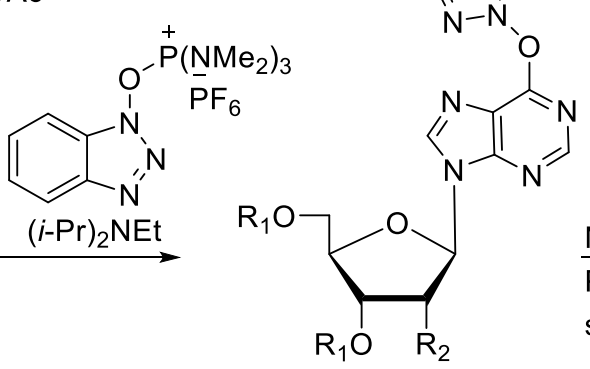

19<smiles></smiles>

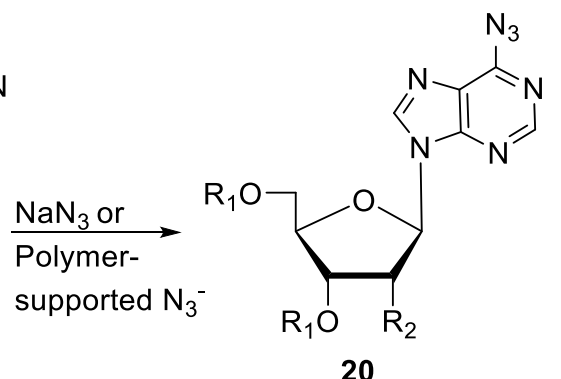

Scheme 6. Synthesis of 6-azidopurine nucleosides from inosine derivatives

Table 2. Conditions for synthesis of 6-azido substituted nucleosides from inosine

\begin{tabular}{|c|c|c|c|c|c|c|}
\hline $\mathrm{R}_{1}$ & TBDMS & TBDMS & $\mathrm{Ac}$ & $\mathrm{Ac}$ & $\mathrm{H}$ & $\mathrm{H}$ \\
\hline $\mathrm{R}_{2}$ & $\mathrm{H}$ & $\mathrm{OTBDMS}$ & $\mathrm{H}$ & $\mathrm{OAc}$ & $\mathrm{H}$ & $\mathrm{OH}$ \\
\hline Reagent & $\mathrm{NaN}_{3}$ & $\mathrm{NaN}_{3}$ & $\mathrm{NaN}_{3}$ & $\mathrm{NaN}_{3}$ & $\begin{array}{c}\text { Polymer } \\
\text { supported, } \\
\mathrm{N}_{3}^{-}\end{array}$ & $\begin{array}{c}\text { Polymer } \\
\text { supported, } \\
\mathrm{N}_{3}^{-}\end{array}$ \\
\hline Solvent & DMSO & DMSO & DMSO & DMSO & $\mathrm{H}_{2} \mathrm{O}$ & $\mathrm{DMF}$ \\
\hline Temp. & $40^{\circ} \mathrm{C}$ & $40^{\circ} \mathrm{C}$ & $\mathrm{Rt}$ & $\mathrm{Rt}$ & $50^{\circ} \mathrm{C}$ & $50^{\circ} \mathrm{C}$ \\
\hline Time & $2 \mathrm{~h}$ & $3.5 \mathrm{~h}$ & $1 \mathrm{~h}$ & $1 \mathrm{~h}$ & $3.5 \mathrm{~h}$ & $5 \mathrm{~h}$ \\
\hline Yields & $99 \%$ & $96 \%$ & $95 \%$ & $75 \%$ & $59 \%$ & $70 \%$ \\
\hline
\end{tabular}

Alternatively, reaction of acetylated inosine analogues $\mathbf{2 1}$ with diphenyl phosphoryl azide (DPPA)/DBU gave acetylated 6-azidopurine nucleosides $\mathbf{2 3}$, with formation of phosphorylated intermediate $\mathbf{2 2}$ (Scheme 7). Tautomerization of 6-azidopurine analogues 
(e.g., 24 to 24a, Figure 21) to their tetrazole counterparts was observed, with concentration of the tetrazolyl isomer increasing with increasing dielectric constant of the solvent. ${ }^{140}$

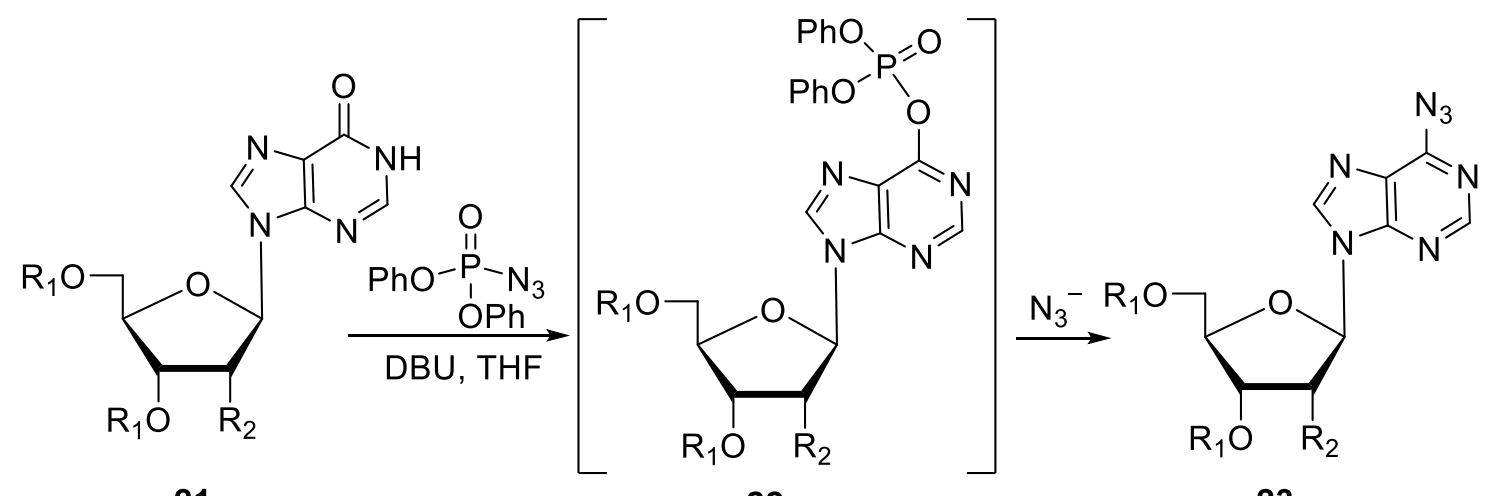

22

23

Scheme 7. Alternative synthesis of 6-azidopurine nucleosides from inosine derivatives

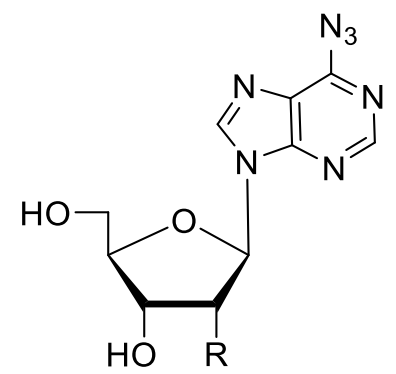

24

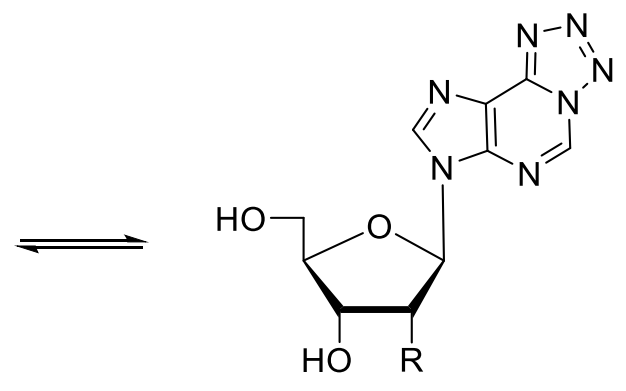

24a

$\mathrm{R}=\mathrm{H}$ or $\mathrm{OH}$

Figure 21. Azido-tetrazole tautomer of 6-azidopurine nucleosides

\subsubsection{Synthesis of azido modified pyrimidine nucleosides}

\subsection{4-Azidopyrimidine nucleosides}

Most literature on synthesis of azido-modified pyrimidine nucleosides focus on synthesis of 4-azidopyrimidine nucleosides, 5-azidouridine , and 5-azidomethyl uridine anlogues. ${ }^{150-153}$ The 4-azidpyrimidine nucleoside analogues have been made for their use as prodrugs for anti-viral and anti-cancer drugs, such as, 1-( $\beta$-D-arabinofuranosyl)cytosine (ara-C), ${ }^{151}$ or as possible therapeutic agents. ${ }^{150}$ Synthesis of 4-azidopyrimidine nucleosides depends on generating a good leaving group at the 4-position of the pyrimidine ring 
followed by replacement with azide. De Napoli et al. achieved this conversion using protected 4-chloro-2'-deoxypyrimidine nucleoside derivative $\mathbf{2 6}$ followed by replacement with azide with $\mathrm{NaN}_{3}$ in anhydrous DMF solvent to give protected 4-tetrazolo-2'deoxypyrimidine nucleoside 27 (Scheme 8). ${ }^{150}$ They found that 27 exists completely in the tetrazole form, even after deprotection, and confirmed this through NMR and UV experiments. $^{150}$

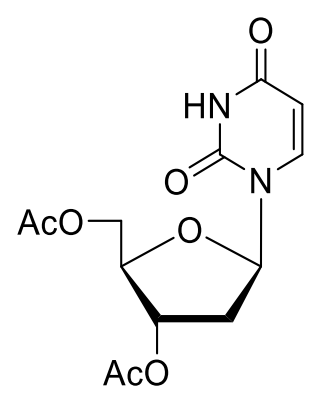

25

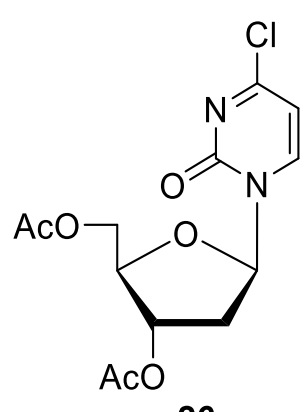

26

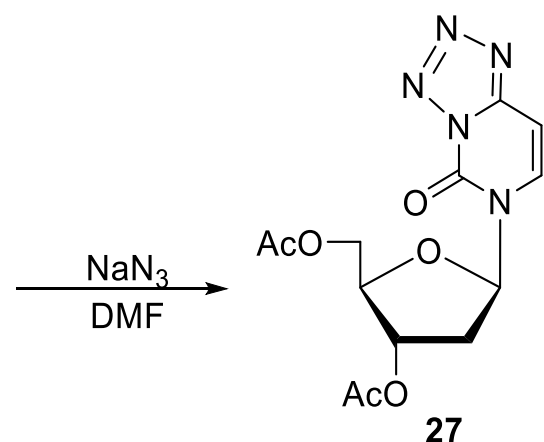

27

Scheme 8. Synthesis of protected 4-tetrazolopyrimidine nucleoside ${ }^{150}$

Alternatively, Kotra et al. synthesized protected 4-azidopyrimidine nucleoside analogue 30 by substitution of the 4-OH of protected arabino uridine $\mathbf{2 8}$ with a 1,2,4triazole leaving group using Divakar-Reese method ( $\mathrm{POCl}_{3} / 1-H-1,2,4$-triazole/Et $\left.3 \mathrm{~N} / \mathrm{ACN}\right)$ to give 29, followed by replacement with azide ( $\mathrm{LiN}_{3} / \mathrm{DMF}$, Scheme 9$)$. They found by NMR, UV, and X-Ray Crystallography studies that cyclization of the azide moiety occurred during deprotection of acetylated azido nucleoside $\mathbf{3 0}$ with $\mathrm{HCl} / \mathrm{MeOH}$ to form tetrazolo product 31. Attempts at deprotection of $\mathbf{3 0}$ with other common methods, such as, $\mathrm{MeOH} / \mathrm{NH}_{3}, \mathrm{MeOH} / \mathrm{NaOMe}$, or $\mathrm{EtOH} / \mathrm{NaCN}$ afforded complete decomposition. The tetrazole compound $\mathbf{3 1}$ did not display any transformation to ara-C during biotransformation studies. ${ }^{154}$ 


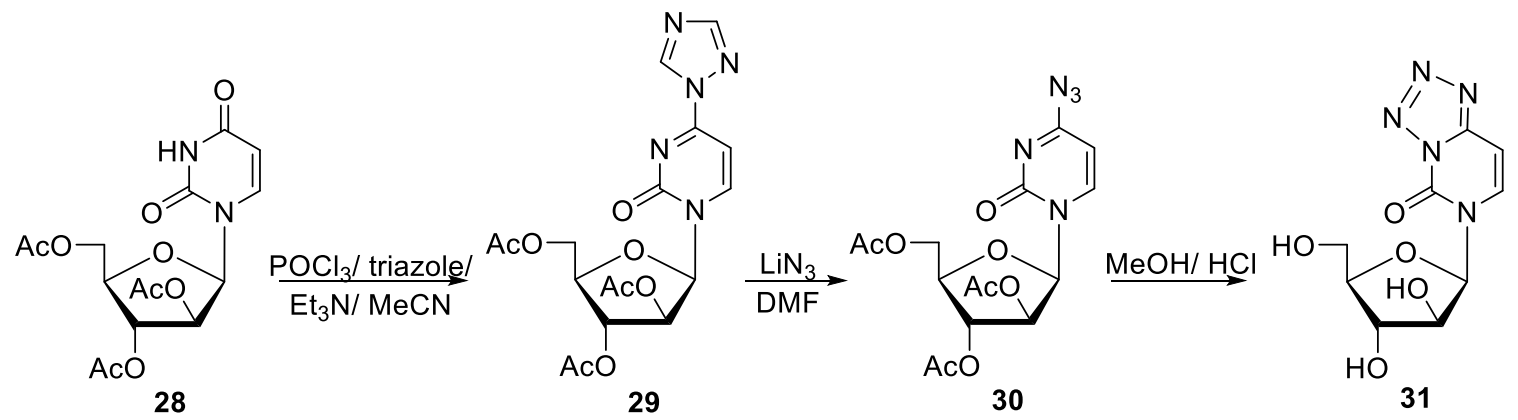

Scheme 9. Synthesis of 4-azido and 4- tetrazolopyrimidine nucleoside from protected arabino uridine $\mathrm{e}^{151}$

\subsection{5-Azidouridine analogues}

The interest in 5-azidouridine analogues (e.g. 35, Scheme 10) arises from their photochemical properties, which can be used in the study of proteins and enzymes. ${ }^{155}$ Synthesis of 5-azido-2'-deoxyuridine was undertaken by nitration of the 5-position of 2'deoxyuridine with nitrosonium tetrafluoroborate, followed by reduction to an amine using $\mathrm{Zn} / \mathrm{HCl}$, and then diazotization/replacement with azide with $\mathrm{NaONO} / \mathrm{HCl} / \mathrm{NaN}_{3} .^{156,157}$ However, the synthesis leads to low overall yield of product 35 (roughly 10-20\%), and requires extensive purification of 5-amino-2'-deoxyuridine analogue (e.g., 34) and use of nitrosonium tetrafluoroborate that is hydroscopic and expensive. ${ }^{152}$ Alternatively, access to 34 can be achieved by treatment of 5-bromouridine with liquid ammonia at high temperature for several days, but that can be tedious and unsafe. ${ }^{158}$

To simplify synthesis of 5-amino-2'-deoxyuridine precursor 34, Gourdain et al. developed a benzylamination/reduction protocol. Benzylamination was achieved using a 5-benzylamine at $90^{\circ} \mathrm{C}$, followed by catalytic hydrogenolysis with ammonium formate $/ 10 \% \mathrm{Pd} / \mathrm{C}$ catalyst to give 34 . Subsequent diazotization/replacement with azide using standard $\mathrm{NaNO}_{2} / 1 \mathrm{~N} \mathrm{HCl} / \mathrm{NaN}_{3}$ at $0^{\circ} \mathrm{C}$ caused cleavage of the glycosidic bond, so 
Gourdain et al. used a milder condition of $\mathrm{NaNO}_{2} / \mathrm{AcOH} / \mathrm{NaN}_{3}$ at $0^{\circ} \mathrm{C}$ to afford $40 \%$ of 5azido-2'-deoxyuridine (AdU) 35. ${ }^{152}$

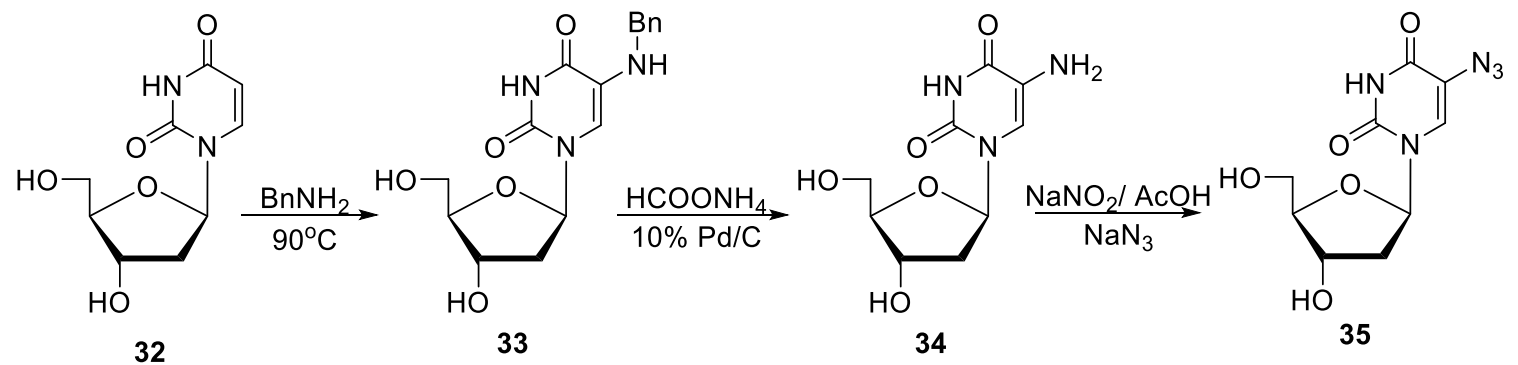

Scheme 10. Gourdain et al. synthesis of 5-azido-2'-deoxyuridine ${ }^{152}$

\subsection{5-Azidomethyl-2'-deoxyuridine analogues}

The 5-azidouridine analogues are quite unstable, decomposing in roughly 4 hours in aqueous media; ${ }^{159}$ however, stable azido-modified pyrimidine nucleoside analogues have been synthesized for the purpose of creating new triazole "click" products. One such analogues is $\mathrm{AmdU}^{153}$ that was later synthesized from protected 2'-deoxythymidine $\mathbf{3 6}$ by bromination of 5- $\mathrm{CH}_{3}$ using $\mathrm{N}$-bromosuccinamide (NBS), $\mathrm{CCl}_{4}$, and azobisisobutyronitrile (AIBN; Scheme 11) to give 37. Then, immediate replacement of bromo with azide was achieved using $\mathrm{NaN}_{3}$ in DMF at high temperature gave protected AmdU 38. ${ }^{160}$ Deprotection of $\mathbf{3 8}$ with tetra- $n$-butylammonium fluoride (TBAF) gave AmdU 39. ${ }^{161}$ Recently, Wen at al. used $\mathbf{3 8}$ as a starting material to synthesize AmdC $\mathbf{4 1}$ by treatment with 2,4,6-triisopropylbenzenesufonyl chloride (TIPBSCl) in the presence of TEA/DMAP to yield an aryl sulfate, which was replaced with ammonia using $\mathrm{NH}_{4} \mathrm{OH}$, and deprotected with TBAF. ${ }^{106}$ 


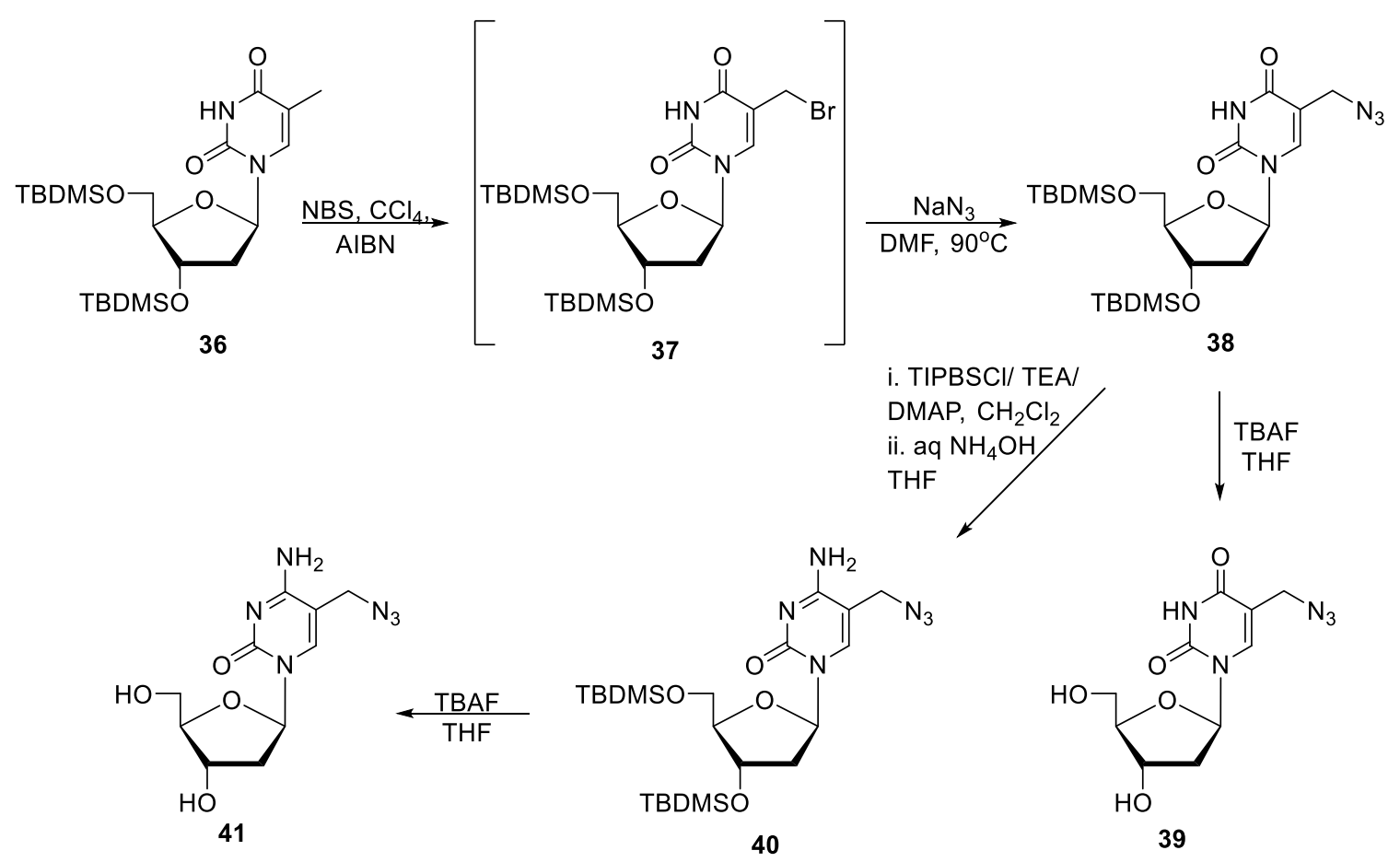

Scheme 11. Synthesis of AmdU 39 and AmdC 41 106,160

\subsubsection{Click-chemistry of azido modified nucleosides}

\subsubsection{Click-chemistry of azido modified purine nucleosides}

Click-chemistry refers to several linking reactions which are simple, high yielding, versatile, and require minimal purification. One example are cycloadditions, or more specifically 1,3-dipolar cycloadditions, such as those which occur between azides and terminal alkynes to yield triazole products. ${ }^{162}$ Formation of triazole-substituted purines by click chemistry has been a large area of research in recent years because of their utility for drug discovery, ${ }^{136,163-165}$ drug delivery, ${ }^{166,167}$ and cellular imaging. ${ }^{134,135,168-171}$

A variety of 2-triazolepurine nucleosides have been synthesized for their use as $A_{3}$ adenosine receptor antagonists, ${ }^{136}$ or as fluorophores for cellular imaging. ${ }^{134,135,171}$ The synthesis of 1,2,3-triazol-1-yl analogues of $N^{6}$-methyladenosine 42 were synthesized using copper-sulfate/sodium ascorbate catalyst, and an alkyne in a water/butanol mixture to give 
43. This solvent system allowed for the precipitation of product from the reaction mixture. $^{136}$
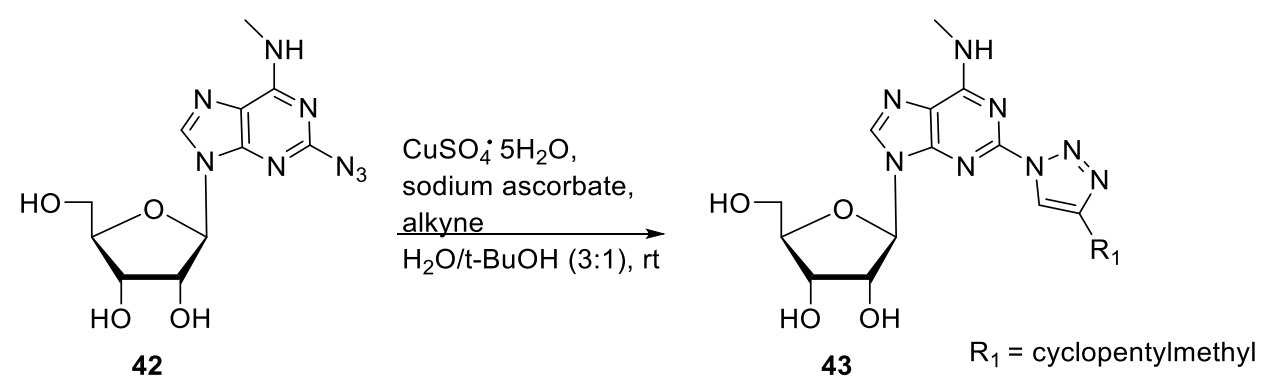

Scheme 12. Synthesis of 2-triazole substituted purine nucleosides

Synthesis of 2-triazolyl derivatives of adenosine were also synthesized using protected 2,6-diazidopurine analogue 44 using two methods: 1) by regioselective reduction of 6azido moiety with $\mathrm{CuSO}_{4}$ /sodium ascorbate catalyst followed by click reaction, or 2) by formation of 2,6-ditriazolyl product, followed by regioselective replacement of 6-triazonyl with aqueous ammonia (Scheme 13). ${ }^{134,171}$ The click-reaction of 2-azido group with alkynes to make 1,2,3-triazoles have good yields regardless of the azido-tetrazole equilibrium seen in 2-azido purines. The use of methanol/water/acetic acid solvent system rather than tert-butanol/water/acetic acid system afforded increased reactivity. Study of the synthesis of compounds $\mathbf{4 4}$ and $\mathbf{4 7}$ by these two methods allowed for the conclusion that $\mathrm{CuSO}_{4} /$ sodium ascorbate catalyst affords first the reduction at $\mathrm{C} 6$ position, although further reaction reduces the $\mathrm{C} 2$ position also (Scheme 14). ${ }^{134}$ 


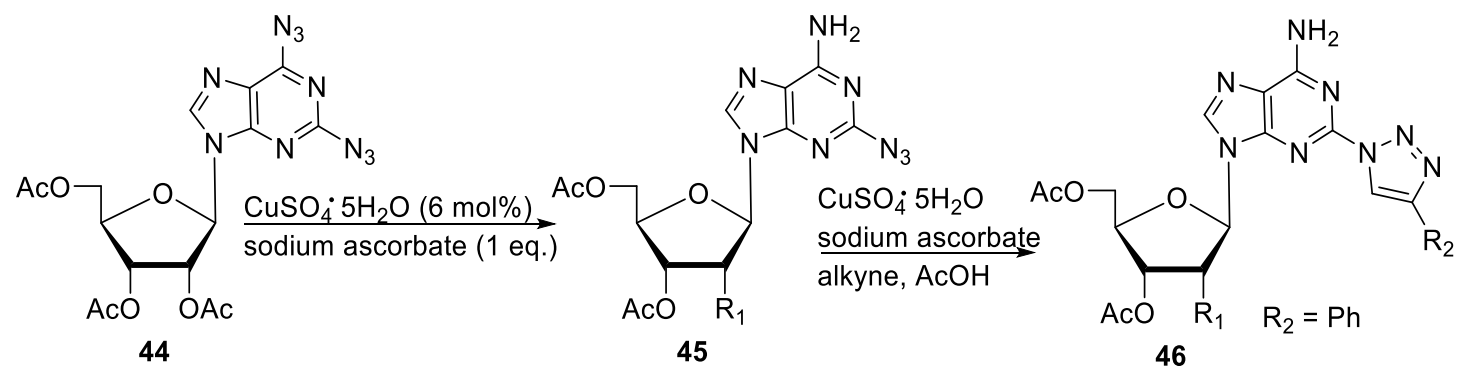

Scheme 13. Regioselective synthesis of 2-triazole substituted adenine nucleosides from 2,6-diazido precursors

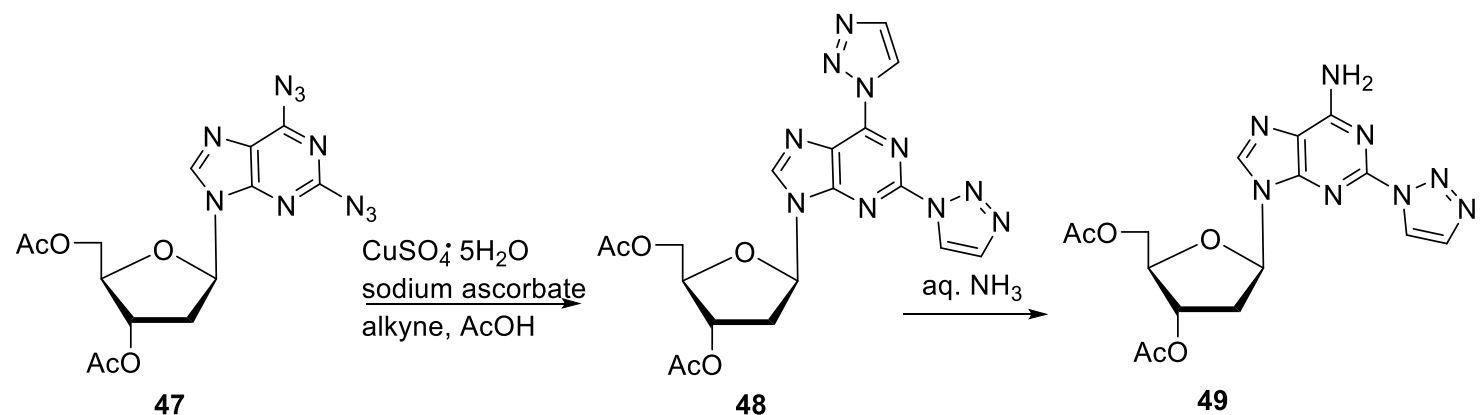

Scheme 14. Synthesis of 2,6-ditriazole substituted purine nucleosides followed by regioselective amination

Extensive studies have been conducted to evaluate the effect of azido-tetrazole tautomerization on the formation of triazole click-chemistry products in protected 6-azido2'-deoxyadenosine 50 (Scheme 15). ${ }^{140}$ Previous literature suggests that low yield for formation of certain triazole products are caused by a shift in the equilibrium towards the tetrazole tautomer (as in 50a). ${ }^{172}$ Synthesis of triazole compound $\mathbf{5 1}$ was attempted using a variety of solvents and several copper-catalysts in order to determine the most successful conditions. It was found that using biphasic (dichloromethane/water) system was imperative for a successful reaction. Furthermore, despite nearly $82 \%$ of tetrazole tautomer 50a (18\% azido tautomer 50) present, during reaction with phenylacetylene in the biphasic system proceeded efficiently. Reduction of the azide functionality to amine was observed 
under several reaction conditions. Table 4 summarizes the results obtained from these studies. ${ }^{140}$

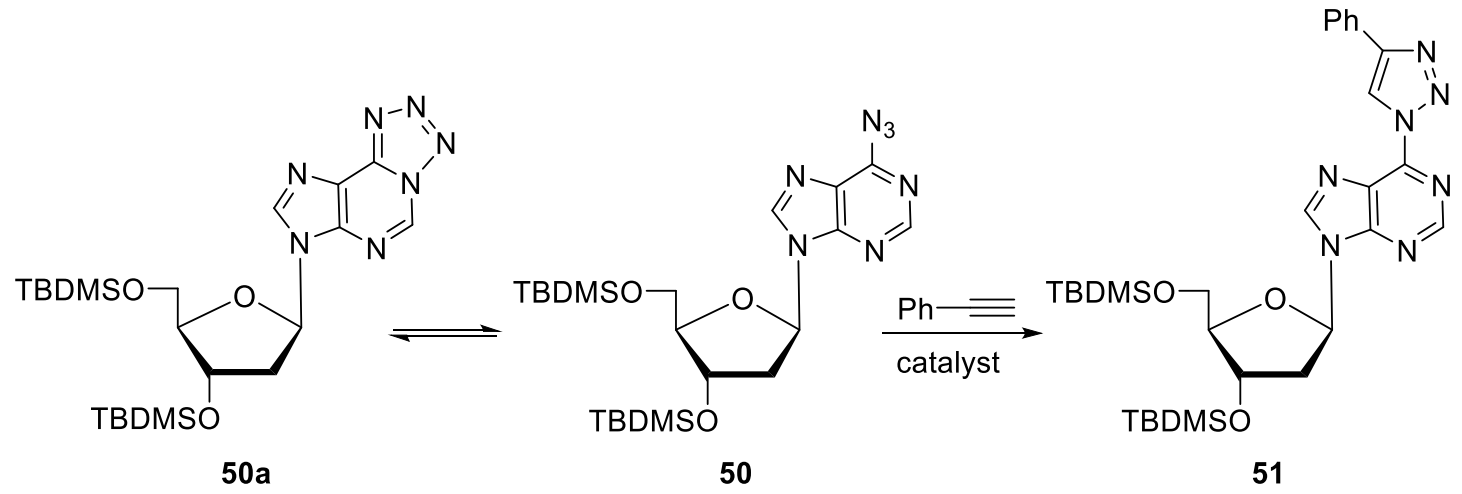

Scheme 15. Azido/tetrazole tautomerization of 6-azidopurine nucleosides and their reactivity towards click- reactions

Table 3. Conditions for the click-reactions of 6-azidopurine nucleosides

\begin{tabular}{|c|c|c|c|}
\hline Catalyst & Solvent & Conditions & $\begin{array}{c}\text { Substrate, Yield, } \\
\text { Reduction }\end{array}$ \\
\hline $\mathrm{CuSO}_{4} / \mathrm{Na}$ ascorbate & $1: 1 t-\mathrm{BuOH} / \mathrm{H}_{2} \mathrm{O}$ & $\mathrm{rt}, 3.5 \mathrm{~h}$ & $63 \%, 26 \%, 11 \%$, \\
\hline $\mathrm{CuSO}_{4} / \mathrm{Na}$ ascorbate & $1: 1 \mathrm{THF} / \mathrm{H}_{2} \mathrm{O}$ & $\mathrm{rt}, 6 \mathrm{~h}$ & $46 \%, 30 \%, 24 \%$ \\
\hline $\mathrm{CuSO}_{4} / \mathrm{Na}$ ascorbate & 1:1 Toulene $/ \mathrm{H}_{2} \mathrm{O}$ & $\mathrm{rt}, 6 \mathrm{~h}$ & $67 \%, 22 \%, 11 \%$ \\
\hline $\mathrm{CuSO}_{4} / \mathrm{Na}$ ascorbate & $1: 1 \mathrm{CHCl}_{3} / \mathrm{H}_{2} \mathrm{O}$ & $\mathrm{rt}, 2 \mathrm{~h}$ & $78 \%$ product \\
\hline $\mathrm{CuSO}_{4} / \mathrm{Na}$ ascorbate & $1: 1 \mathrm{CH}_{2} \mathrm{Cl}_{2} / \mathrm{H}_{2} \mathrm{O}$ & $\mathrm{rt}, 3.5 \mathrm{~h}$ & $81 \%$ product \\
\hline $\mathrm{Cu}(\mathrm{Phen})\left(\mathrm{PPh}_{3}\right) \mathrm{Br}$ & $1: 1 \mathrm{CH}_{2} \mathrm{Cl}_{2} / \mathrm{H}_{2} \mathrm{O}$ & $\mathrm{rt}, 24 \mathrm{~h}$ & $46 \%$ product \\
\hline $\mathrm{Cu}\left(\mathrm{PPh}_{3}\right) \mathrm{Br}$ & $1: 1 \mathrm{CH}_{2} \mathrm{Cl}_{2} / \mathrm{H}_{2} \mathrm{O}$ & $\mathrm{rt}, 15 \mathrm{~h}$ & $73 \%$ product \\
\hline CuIMes & $1: 1 \mathrm{CH}_{2} \mathrm{Cl}_{2} / \mathrm{H}_{2} \mathrm{O}$ & $\mathrm{rt}, 20 \mathrm{~h}$ & $60 \%, 29 \%, 11 \%$ \\
\hline $\mathrm{CuIPr}$ & $1: 1 \mathrm{CH}_{2} \mathrm{Cl}_{2} / \mathrm{H}_{2} \mathrm{O}$ & rt, $24 \mathrm{~h}$ & NR \\
\hline $\mathrm{CuCl}$ & $1: 1 \mathrm{CH}_{2} \mathrm{Cl}_{2} / \mathrm{H}_{2} \mathrm{O}$ & $\mathrm{rt}, 5 \mathrm{~h}$ & $76 \%$ product \\
\hline $\mathrm{CuTC}$ & $1: 1 \mathrm{CH}_{2} \mathrm{Cl}_{2} / \mathrm{H}_{2} \mathrm{O}$ & $\mathrm{rt}, 6 \mathrm{~h}$ & $63 \%$ product \\
\hline $\begin{array}{c}\mathrm{Cu} / \mathrm{C}+2 \text { equiv. } \\
\mathrm{Et}_{3} \mathrm{~N}\end{array}$ & 1,4-dioxane & $\begin{array}{c}\mathrm{rt}(15 \mathrm{~h}), 60^{\circ} \mathrm{C} \\
(1.5 \mathrm{~h})\end{array}$ & $0 \%, 51 \%, 38 \%$ \\
\hline $\mathrm{Cu} / \mathrm{C}$ & 1,4-dioxane & $\mathrm{rt}, 23 \mathrm{~h}$ & NR \\
\hline $\mathrm{Cu} / \mathrm{C}$ & $1: 1 \mathrm{CH}_{2} \mathrm{Cl}_{2} / \mathrm{H}_{2} \mathrm{O}$ & $\mathrm{rt}, 2.5 \mathrm{~h}$ & NR \\
\hline
\end{tabular}

Synthesis of triazole products by strain-promoted click-chemistry from 8azidoadenosine derivatives was achieved using cyclopropyl cyclooctyne (OCT) in a 
mixture of acetonitrile/water (3:1) at ambient temperature (Scheme 16). High yield was achieved for these reactions (above 90\%) in methanol, ethanol, or Opti-MEM I cell culture media with roughly $60 \%$ conversion within 20 minutes. ${ }^{135}$ Addition of bulky triazole substituents in the 8-position of adenosine forces the molecule into a predominantly syn confirmation because of the electrostatic repulsion between the 8-position substituent and the ribose moeity. ${ }^{173,174}$ Triazoles $\mathbf{5 3}$ possess moderate fluorescence emissions and were utilized for cellular imaging. ${ }^{135}$

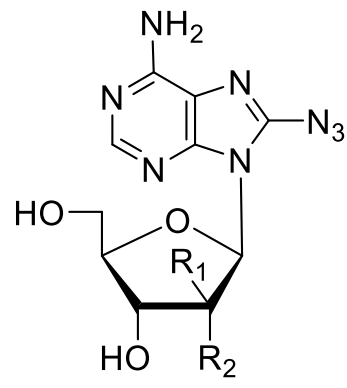

$52 \mathrm{R}_{1}=\mathrm{H}, \mathrm{R}_{2}=\mathrm{OH}$

52a $\mathrm{R}_{1}=\mathrm{OH}, \mathrm{R}_{2}=\mathrm{H}$

52b $R_{1}=F, R_{2}=H$

52c $R_{1}=H, R_{2}=H$

\section{$\frac{\text { Cyclo-octyne }}{\text { "Click" }}$}

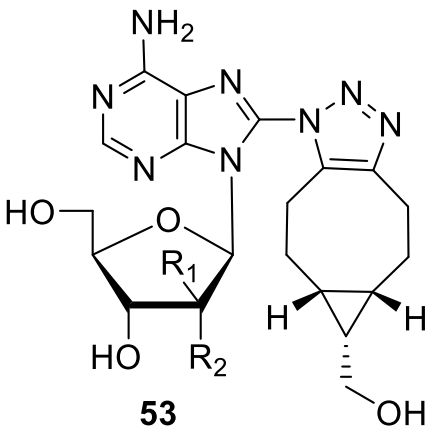

53

Scheme 16. SPAAC "click" reactions between azido nucleosides and cyclooctyne

\subsubsection{Click-chemistry of azido modified pyrimidine nucleosides}

A variety of articles have been published in which pyrimidine nucleosides are modified with alkyl chains bearing a terminal alkyne that can be reacted with azido-modified molecules. ${ }^{175-181}$ However, our focus in the present section will be the formation of triazole adducts from pyrimidine nucleosides modified with azide at the base part and their reaction with alkynes. Two types of click-reaction are usually undertaken with azido-modified pyrimidine nucleosides for the purpose of cellular imaging; copper(I)-catalyzed azidealkyne cycloaddition $(\mathrm{CuAAC})^{182}$ or strain-promoted azide-alkyne cycloaddition (SPAAC). ${ }^{168}$ 
In 2014, Neef et al. were able to metabolically incorporate azido modified nucleosides onto living cells and perform cycloadditions with fluorescent alkynes, which allowed for sensitive detection of DNA replication. These studies focused mainly on catalyst free SPAAC reactions in vivo. The 5-modified pyrimdine nucleoside derivative are compatible with cellular enzymes for DNA incorporation but click-chemistry of 5-azido-2'deoxyuridine is particularly troublesome given the short stability of the compound in aqueous media. Because of this use of 5-(azidomethyl)-2'-deoxyuridine (AmdU) was used because it is stabile in physiological conditions.

The AdU and AmdU samples were incubated with HeLa cells that express low-fidelity thymidine kinase for 24 hours, followed by treatment with AlexaFluor 488 (Figure 22) or bicyclo[6.1.0]nonyne (BCN) AlexaFluor 488 alkyne in the presence of copper(I) in order to form CuAAC or SPAAC "click" products. The cells were then imaged, and compared with 4',6-diamidino-2-phenylindole (DAPI) stained cells. Cells incubated with AdU could not be detected with imaging, but the ones treated with AmdU were detected. ${ }^{161}$ The Luedtke group then did studies on phosphotriester AmdU derivatives' reaction with AlexaFluor 594 alkyne and their incorporation onto wild-type HeLa cells and animals. The 5'-bispivaloyloxymethyl (POM) AmdU derivatives showed in Figure 23 displayed the best chemical stability, metabolic incorporation, and lower toxicity. ${ }^{183}$ 


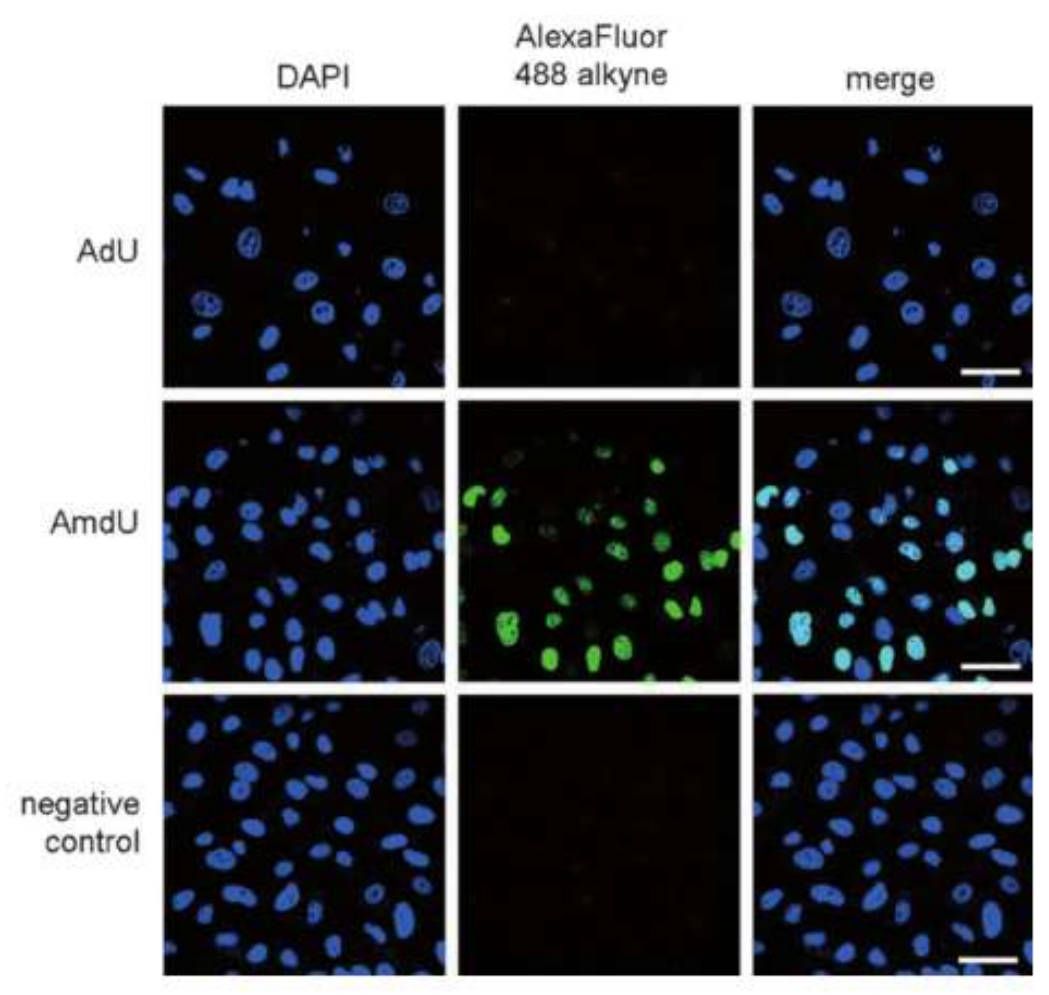

Figure 22. AdU and AmdU images after incubation with AlexaFluor 488 alkyne $^{161}$

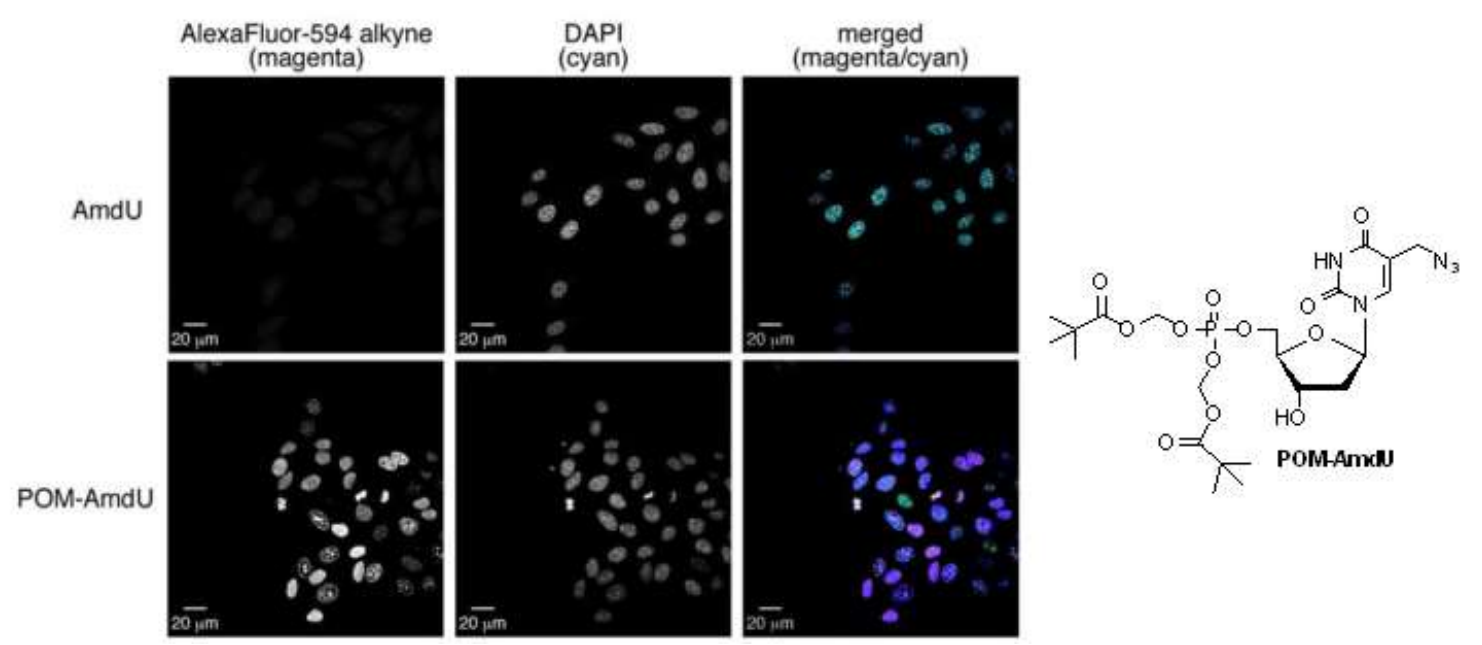

Figure 23. POM-AmdU images after incubation with AlexaFluor 594 alkyne ${ }^{183}$

Similarly, Ren et al. incorporated 5-azidomethyl dUTP and 5-trans-cyclooctyne dUTP analogues onto DNA by primer extension, reverse-transcription and PCR amplification. They found that 5-azidomethyl dUTP was a better substrate for incorporation than the 5trans-cyclooctyne dUTP analogues, but cycloaddition reaction between the cyclooctyne 
and tetrazine Cy3-dye was more efficient than SPAAC reaction with 55 (Scheme 17). ${ }^{184}$ Zayas et al. showed efficient synthesis of triazole "click" products by SPAAC reaction with 5-azido-2'-deoxyuridine. The resulting cyclooctyne nucleosides displayed moderate fluorescent properties. ${ }^{135}$

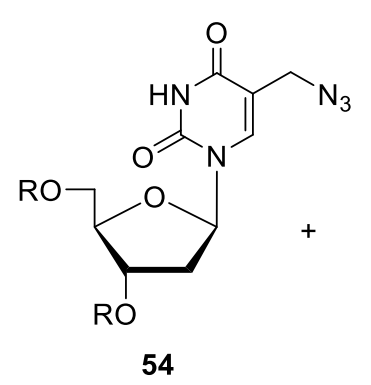

$\mathrm{R}=\mathrm{DNA}$ strand
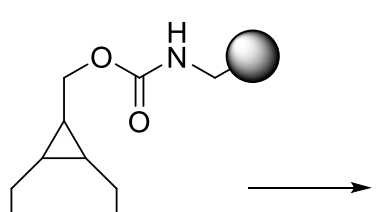

Ro

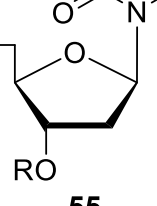

55

Scheme 17. Synthesis of 5-trans-cyclooctyne dUTP analogues ${ }^{184}$

\subsubsection{Azido-tetrazole chemistry}

A tetrazole is a five-membered heterocyclic ring containing four nitrogen atoms and one carbon atom. The tetrazole ring contains $6 \pi$-electrons in a planar confirmation with bond lengths similar to that of benzene. It possesses strong electron-withdrawing properties, making it deactivating as a substituent on benzene. ${ }^{185}$ Tetrazoles are very important because of their varied applications, including serving as, lipophilic spacers in pharmaceuticals, in specialty explosives, in photography systems, and as a precursor to many nitrogen containing heterocycles. ${ }^{186}$

Tetrazoles are often formed from an imine in which double bonded carbon is substituted by an azido group. Several factors affect whether these types of molecules are found as cyclic tetrazoles or acyclic azides. ${ }^{187}$ For example, the type of substituent $\left(\mathrm{R}\right.$ or $\mathrm{R}^{1}$, Figure 24) effects the equilibrium since electron-withdrawing substituents tend to favor the azide 
form, while electron donating substituents stabilize ring closure. Another factor affecting the equilibrium is temperature, since cleavage of the tetrazole ring is an endothermic process; meaning the azide form is favored at higher temperatures. Finally, basic solvents strongly favor the tetrazole form by increasing electron density of the carbon-nitrogen double bond, while acidic solvents favor the azide form through electron depletion. Generally, polar solvents tend to favor the tetrazole form, while non-polar solvents lean the equilibrium towards the azide. ${ }^{187}$

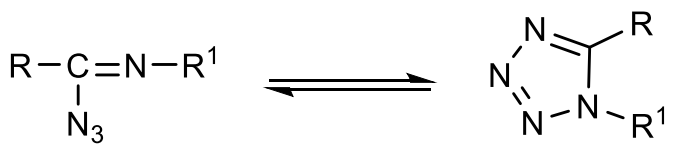

Figure 24. Azido-tetrazole equilibrium ${ }^{187}$

Tetrazoles and their tetrazolinyls radicals have been studied for many years because of their stability. ${ }^{188}$ The EPR has shown that tetrazolinyl radicals remain in the cyclic confirmation, and not as the open-chain isomers. ${ }^{188-190}$ These radicals are so stable that they do not interact with oxygen at ambient conditions and their inertness is further increased by electron-withdrawing properties of substituents at $\mathrm{R}^{1}$ (Figure 20). ${ }^{191}$ Under weakly acidic conditions, the tetrazolinyl radicals undergo decomposition to form either tetrazoles or open-chain formazans. ${ }^{191}$

The azido-tetrazole tautomerization has also been studied in depth in purines and pyrimidines. ${ }^{192-196}$ It has been found that the electron-density of the imino nitrogen pair and steric effects are important factors, aside from temperature and solvent, in the ratio of either isomer. ${ }^{197}$ In Figure 25, the different tautomeric forms of 2-azidopurines and 4azidopyrimidines is shown. The percentage in solution of each tautomer was found to be dependent on the solvent, as well as, properties of $\mathrm{R}^{1}$ and/or $\mathrm{R}^{2}$ substitution [electron 
withdrawing group (EWG), electron donating group (EDG), sterically demanding group $(\mathrm{SDG})]{ }^{192-196}$

$\mathrm{R}^{1}=\mathrm{OH}$ or $\mathrm{NH}_{2}$<smiles>[R]c1nc2nnnn2c2[nH]cnc12</smiles>

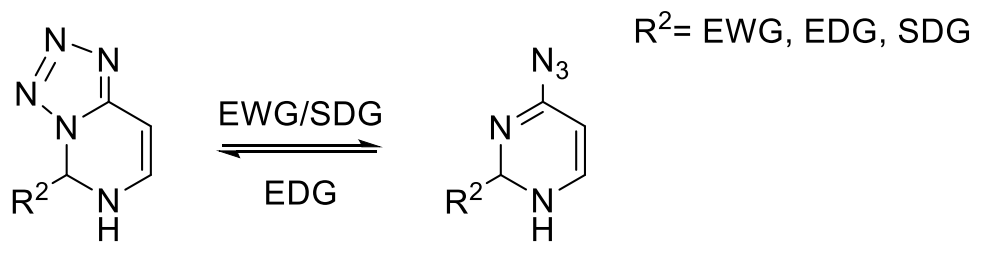

Figure 25. Azido-tetrazole equilibria in 2-azidopurines and 4-azidopyrimidines ${ }^{194,196}$

\subsubsection{Azido/tetrazole tautomerization of 4-azidopyrimidine nucleosides}

For 4-azidopyrimidine nucleosides, there is only one feasible confirmation in which tetrazole tautomerization can occur (e.g., T; Figure 26), and it was found to be the predominant tautomer, as seen through UV and ${ }^{1} \mathrm{H} /{ }^{13} \mathrm{C}$ NMR data. The ${ }^{1} \mathrm{H}$ NMR data showed that the protons in the tetrazole form were more deshielded, causing peaks from H5 and H6 protons to have chemical shifts closer together than would be expected for the corresponding azide tautomer. A similar effect is seen in ${ }^{13} \mathrm{C}$ NMR. ${ }^{130,150,196}$ The tetrazole isomer, in this case, is so stable that it was found to remain intact even under acidic $\mathrm{pH}$ ( 2). Moreover, at a basic $\mathrm{pH}(\sim 11)$, analogue $\mathbf{T}$ was found to form a 2',6-anhydro linkage. $^{151}$

Interestingly, 4-azido substitution in a 2',3',5'-tri- $O$-acetyl protected arabino pyrimidine nucleoside is found in the acyclic azido form (e.g., S; Figure 26). However, when deprotected using $\mathrm{HCl} / \mathrm{MeOH}$, the tetrazole isomer (e.g., T) is exclusively formed. 
To confirm which isomer was formed, UV data acquired in methanol showed $\lambda_{\max }$ for the azido form at $266 \mathrm{~nm}$ and $255 \mathrm{~nm}$, and $\lambda_{\max }$ for the tetrazole form at $267.5 \mathrm{~nm}$ and 257.5

nm. Moreover, for 4-azido-5'-O-benzoyl-2',3'-dideoxy-2-fluoro- $\beta$-D-arabinofuranosyl derivative, deprotection with $\mathrm{MeOH} / \mathrm{NH}_{3}$ provided the tetrazole tautomer without decomposition. ${ }^{130}$

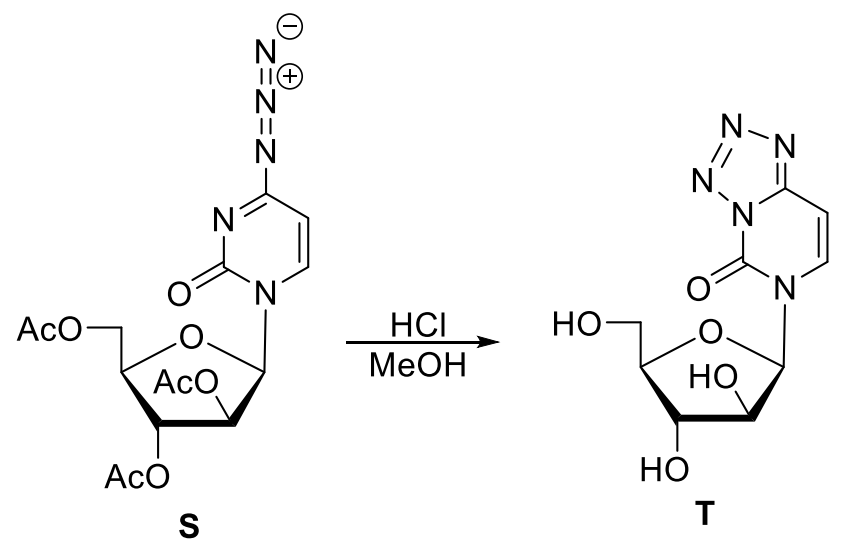

Figure 26. Azido-tetrazole tautomerization in 4-azidopyrimidine nucleosides

The azido/tetrazole moiety is considered a latent amino functionality, which is interesting in the study of photosensitizing ${ }^{198,199}$ or one-electron oxidation of nucleobases. Considering that azido-modified nucleosides cannot be incorporated onto DNA by conventional solid-phase nucleotide synthesis due to the Staudinger reaction between the azide and phosphoramidite $[\mathrm{P}(\mathrm{III})]$ units, it is interesting and important to study the incorporation of tetrazole nucleoside analogues onto DNA. ${ }^{142}$ Some methods have been developed by which to incorporate azido-modified nucleosides onto DNA, yet these methods remain to be developed for large scale use. ${ }^{200}$ Tetrazole derivatives; however, are highly stable and will not react with the P(III) moiety. Recent literature reports the synthesis of a dinucleotide containing two 4-tetrazolopyrimidine nucleotides (Figure 27). ${ }^{199}$ 


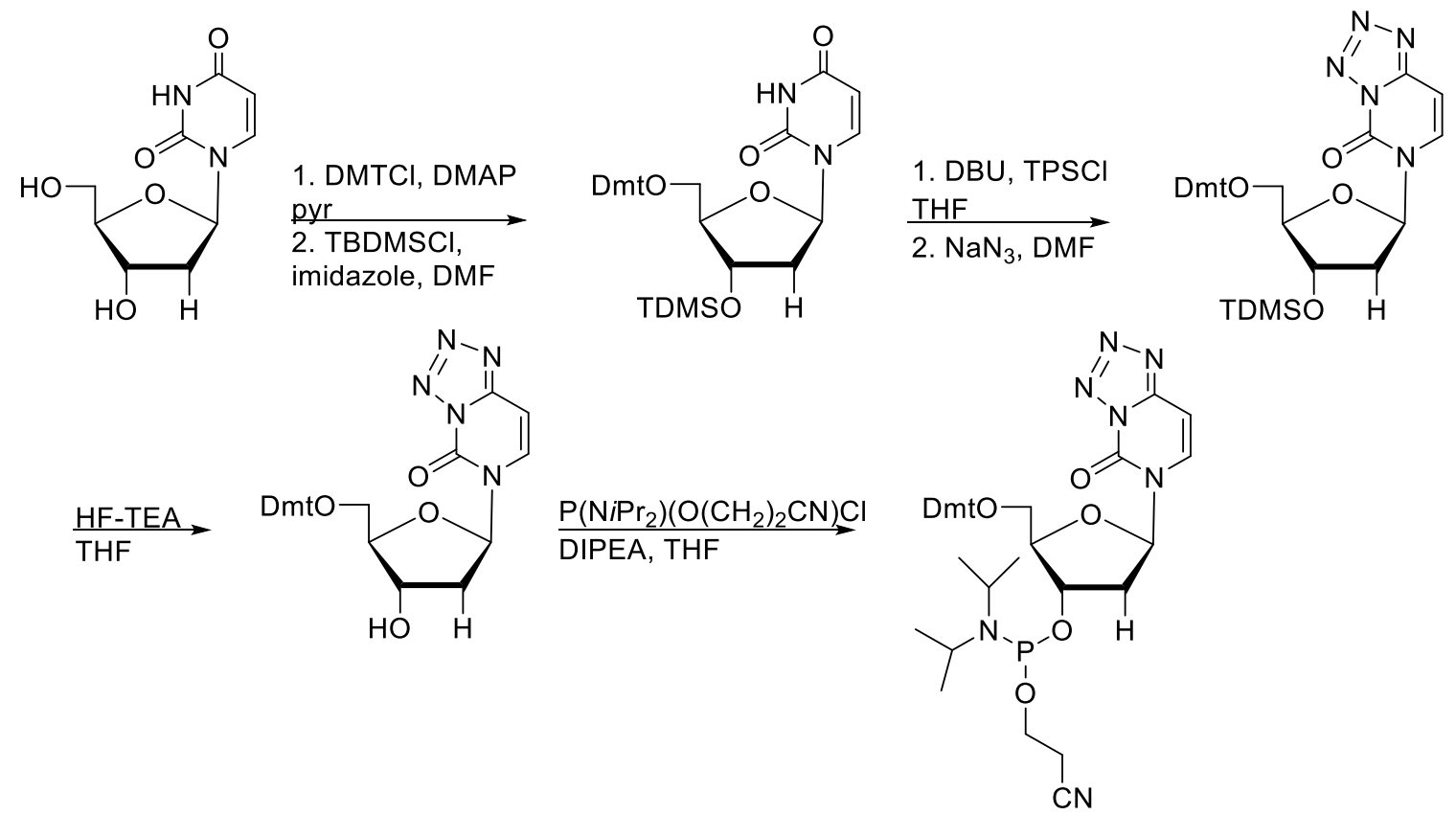

Figure 27. Synthesis of phosphoramidite precursor for the solid phase synthesis containing 4-tetrazolo pyrimidine base ${ }^{199}$

Tetrazole nucleosides have been studied extensively for their photochemical properties. In 2006, Dr. Clivio's group studied the photochemical base expansion of 4-tetrazolo pyrimidines, finding that they form useful 1,3,5-triazepin-2,4-dione nucleosides under aqueous conditions. These "fat pyrimidine" analogues are not only useful as therapeutic agents, but also as probes for the biological study of nucleic acids. ${ }^{198}$ The di(4tetrazolouracil) dinucleoside monophosphate has also been studied for the formation of dicytosine cyclobutene product, which is known to be responsible for DNA mutation in skin cancers. ${ }^{199}$

\subsubsection{Azido/tetrazole tautomerization of azidopurine nucleosides}

For azido-modified purine nucleosides, formation of cyclic tetrazole tautomers is possible with both N1 and N3 positions. In Figure 28, several azidopurine nucleosides are shown, along with their possible tetrazole isomers. The azido-tetrazole isomers of 6- 
azidopurine nucleosides have been reported, and studied extensively for their ratios in varied solvents. ${ }^{134,140}$ Both 2-azidoadenosine and 2-azidoinosine analogues have been studied, yet the formation of the tetrazole isomer has only been reported for adenosine-type analogues. ${ }^{141,143,146,171}$ Moreover, 8-azidoadenosine and 8-azidoguanosine have been synthesized for a variety of applications, yet their tetrazole isomers have not been described. ${ }^{135,201}$

2-position modified
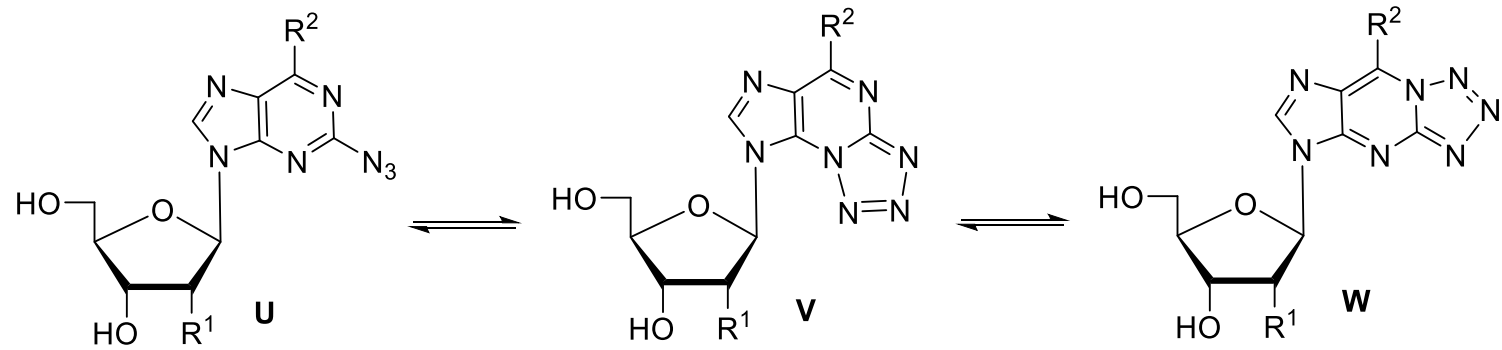

6-position modified
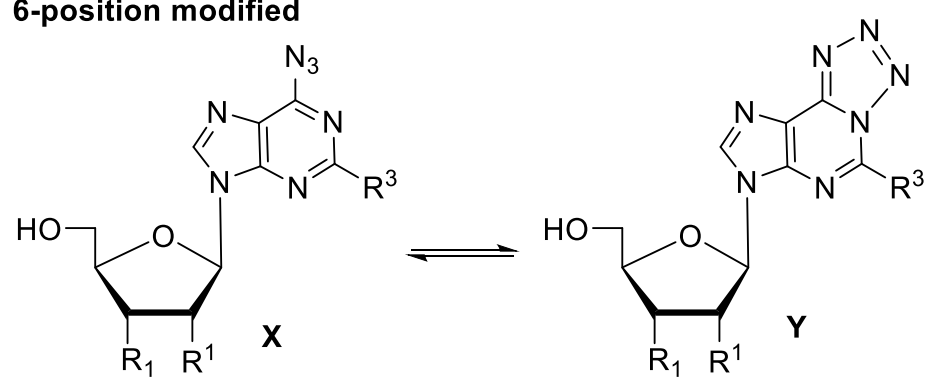

$$
\begin{aligned}
& \mathrm{R}^{1}=\mathrm{OH} \text { or } \mathrm{H} \\
& \mathrm{R}^{2}=\mathrm{NH}_{2} \text { or } \mathrm{OH} \\
& \mathrm{R}^{3}=\mathrm{H} \text { or } \mathrm{NH}_{2}
\end{aligned}
$$

8-position modified

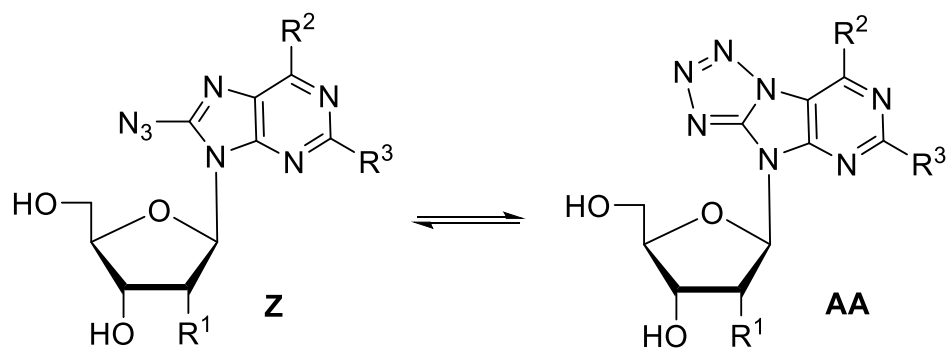

Figure 28. Azido-tetrazole isomers in 2-, 6-, 8-azido substituted purine nucleosides

The 2-azidoadenosine analogues can potentially exhibit two tetrazole tautomerizations, one pertaining to the $\mathrm{N} 3$ position, as in $\mathbf{V}$, and one with the $1 \mathrm{~N}$ position, as in $\mathbf{W}$ (Figure 
28). Although 2-azidoadenine analogue has been reported to exist in all three analogous forms (azido $\mathbf{U}$, tetrazole $\mathbf{V}$ and $\mathbf{W}$ ), ${ }^{195}$ 2-azidoadenosine and 2-azido-2'-deoxyadenosine only exists in forms $\mathbf{U}$ and $\mathbf{W} .{ }^{141,142}$ Presence of analogues $\mathbf{U}$ and $\mathbf{W}$ were confirmed by ${ }^{1} \mathrm{H}$ NMR (Table 4), where the anomeric protons (H1') were found to be relatively close together with only $0.14 \mathrm{ppm}$ difference. If the tetrazole form $\mathbf{V}$ was present, the proximity of the tetrazole to the anomeric proton would have caused significant deshielding. ${ }^{142}$
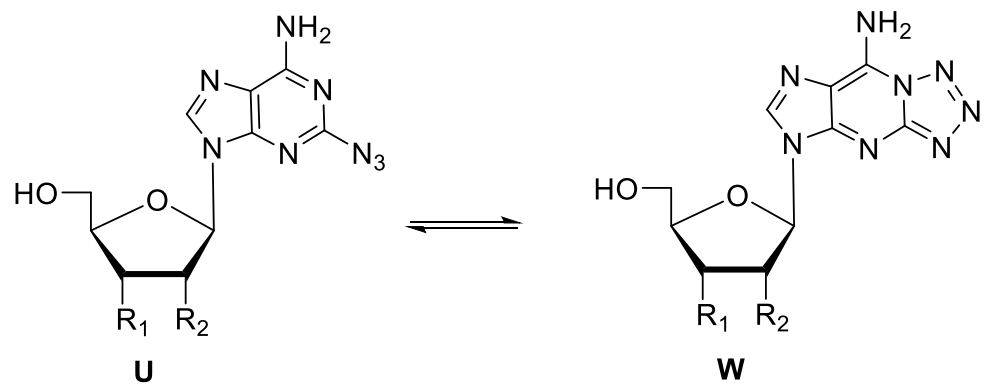

Table 4. ${ }^{1} \mathrm{H}$ NMR Chemical Shifts for Azido/Tetrazole Tautomers of 2-azidopurine nucleosides in Deuterated Solvents

\begin{tabular}{|c|c|c|c|c|c|c|c|c|}
\hline $\mathrm{R}_{1}$ & \multicolumn{2}{|c|}{$\mathrm{OH}$} & \multicolumn{2}{c|}{$\mathrm{OH}$} & \multicolumn{2}{c|}{$\mathrm{H}$} & \multicolumn{2}{c|}{ Dehydro } \\
\hline $\mathrm{R}_{2}$ & \multicolumn{2}{|c|}{$\mathrm{OH}$} & \multicolumn{2}{c|}{$\mathrm{H}$} & \multicolumn{2}{c|}{$\mathrm{H}$} & \multicolumn{2}{c|}{ Dehydro } \\
\hline Position & 6- $\mathrm{NH}_{2}$ & $\mathbf{8}-\mathrm{H}$ & $\mathbf{6}-\mathrm{NH}_{2}$ & $\mathbf{8}-\mathrm{H}$ & $\mathbf{6}-\mathrm{NH}_{2}$ & $\mathbf{8}-\mathrm{H}$ & 6- $\mathrm{NH}_{2}$ & 8-H \\
\hline Form U & 7.67 & 8.28 & 7.60 & 8.25 & 7.60 & 8.29 & 7.58 & 8.08 \\
\hline Form W & 9.46 & 8.56 & 9.40 & 8.53 & 9.40 & 8.59 & 9.39 & 8.35 \\
\hline$\Delta \delta$ & 1.79 & 0.28 & 1.80 & 0.28 & 1.80 & 0.30 & 1.81 & 0.27 \\
\hline Ratio U/W & \multicolumn{6}{|c|}{$67: 33$} & \multicolumn{6}{c|}{$65: 35$} & \multicolumn{3}{c|}{$67: 33$} & \multicolumn{3}{c|}{$66: 34$} \\
\hline
\end{tabular}

The $\mathrm{C6}$ azidopurine nucleosides exhibit tautomerization as shown in Figure $28(\mathbf{X} \rightarrow$ Y). The ratio of isomer formation in different solvents and temperature has been studied (Table 5). These studies found, similarly to those with other azidopurine derivatives, that the isomers could be easily identified by the deshielding effects of the tetrazole moiety on the $\mathrm{H} 2$ and $\mathrm{H} 8$ signals in ${ }^{1} \mathrm{H}$ NMR. At higher temperatures, azido analogues were found primarily in the azido isomer $(\mathbf{X})$, while when dissolved in polar solvents the azido analogues were found primarily in the tetrazole isomer $(\mathbf{Y}) .{ }^{139}$ 

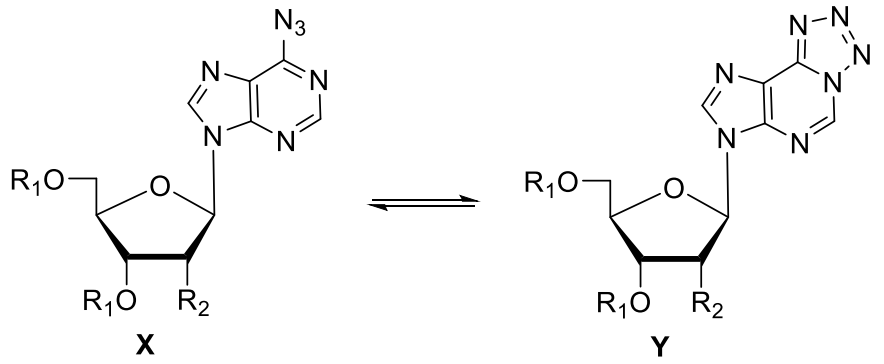

Table 5. ${ }^{1} \mathrm{H}$ NMR Chemical Shifts for Azido/Tetrazole Tautomers of 6-azidopurine nucleosides in Deuterated Solvents

\begin{tabular}{|c|c|c|c|c|c|c|c|c|c|c|c|c|}
\hline $\mathrm{R}_{1}$ & \multicolumn{3}{|c|}{ Ac } & \multicolumn{3}{|c|}{ Ac } & \multicolumn{3}{|c|}{$\mathrm{H}$} & \multicolumn{3}{|c|}{$\mathrm{H}$} \\
\hline $\mathrm{R}_{2}$ & \multicolumn{3}{|c|}{$\mathrm{H}$} & \multicolumn{3}{|c|}{ OAc } & \multicolumn{3}{|c|}{$\mathrm{H}$} & \multicolumn{3}{|c|}{$\mathrm{OH}$} \\
\hline Peak & $\mathrm{H} 2$ & $\mathrm{H} 8$ & $\mathrm{H} 1^{\prime}$ & $\mathrm{H} 2$ & $\mathrm{H} 8$ & H1' & $\mathrm{H} 2$ & H8 & H1' & $\mathrm{H} 2$ & H8 & H1' \\
\hline $\mathrm{CDCl}_{3}(\mathrm{~A})$ & 8.67 & 8.18 & 6.47 & 8.68 & 8.15 & 6.21 & - & - & - & - & - & - \\
\hline $\mathrm{CDCl}_{3}(\mathrm{~T})$ & 9.53 & 8.40 & 6.59 & 9.54 & 8.38 & 6.33 & - & - & - & - & - & - \\
\hline Ratio (A/T) & \multicolumn{3}{|c|}{$56.9: 43.1$} & \multicolumn{3}{|c|}{ 61.9:38.1 } & \multicolumn{3}{|c|}{-} & \multicolumn{3}{|c|}{-} \\
\hline THF- $d_{8}(\mathrm{~A})$ & 8.60 & 8.35 & 6.48 & 8.62 & 8.35 & 6.27 & 8.57 & 8.46 & 6.48 & - & - & - \\
\hline THF- $d_{8}(\mathrm{~T})$ & 9.81 & 8.52 & 6.62 & 9.83 & 8.52 & 6.41 & 9.78 & 8.68 & 6.64 & - & - & - \\
\hline Ratio (A/T) & \multicolumn{3}{|c|}{$14.1: 85.9$} & \multicolumn{3}{|c|}{$16.9: 83.1$} & \multicolumn{3}{|c|}{$15.1: 84.9$} & \multicolumn{3}{|c|}{ - } \\
\hline $\mathrm{CD}_{2} \mathrm{Cl}_{2}(\mathrm{~A})$ & 8.65 & 8.19 & 6.48 & 8.67 & 8.16 & 6.22 & - & - & - & - & - & - \\
\hline $\mathrm{CD}_{2} \mathrm{Cl}_{2}(\mathrm{~T})$ & 9.54 & 8.42 & 6.60 & 9.56 & 8.41 & 6.36 & - & - & - & - & - & - \\
\hline Ratio (A/T) & \multicolumn{3}{|c|}{$27.5: 72.5$} & \multicolumn{3}{|c|}{$31.4: 68.6$} & \multicolumn{3}{|c|}{-} & \multicolumn{3}{|c|}{ - } \\
\hline Acetone- $d_{6}(\mathrm{~A})$ & 8.68 & 8.53 & 6.57 & 8.70 & 8.53 & 6.36 & - & - & - & - & - & - \\
\hline Acetone- $d_{6}(\mathrm{~T})$ & 9.95 & 8.71 & 6.73 & 9.97 & 8.71 & 6.52 & - & - & - & - & - & - \\
\hline Ratio (A/T) & \multicolumn{3}{|c|}{$6.2: 93.8$} & \multicolumn{3}{|c|}{ 7.6:92.4 } & \multicolumn{3}{|c|}{-} & \multicolumn{3}{|c|}{-} \\
\hline DMSO- $d_{6}(\mathrm{~A})$ & - & - & - & - & - & - & - & - & - & - & - & - \\
\hline DMSO- $d_{6}(\mathrm{~T})$ & 10.2 & 8.87 & 6.61 & 10.2 & 8.88 & 6.46 & 10.1 & 8.88 & 6.57 & 10.2 & 8.93 & 6.15 \\
\hline Ratio (A/T) & \multicolumn{3}{|c|}{$0.9: 99.1$} & \multicolumn{3}{|c|}{ 1.9:98.1 } & \multicolumn{3}{|c|}{$0.1: 99.9$} & \multicolumn{3}{|c|}{$0.9: 99.1$} \\
\hline
\end{tabular}




\section{RESEARCH OBJECTIVES}

The first aim of this dissertation is the synthesis and evaluation of nucleoside analogues which can serve as substrates for incorporation of radionuclei suitable for Positron Emission Tomography imaging. The objective is to synthesize two classes of gemcitabine analogues with lipophilic $4-N$-alkyl chain i) bearing $\beta$-keto sulfonate moiety for subsequent ${ }^{18}$ F-fluorination (e.g., 56; Figure 29), and ii) conjugated to SCN-Bn-NOTA chelator for ${ }^{68} \mathrm{Ga}$ complexation (e.g., 65).
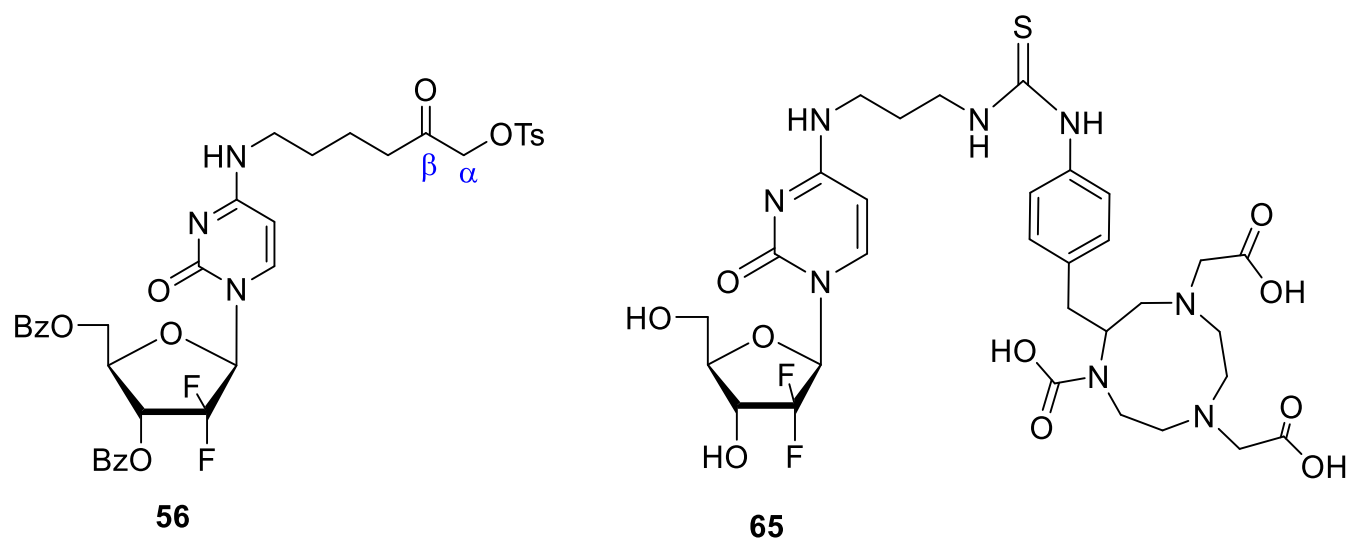

Figure 29. Proposed 4- $N$-alkyl gemcitabine analogues suitable for radiolabeling

The targeted 4- $\mathrm{N}$-alkyl analogs should increase lipophilicity of gemcitabine, which can increase uptake into the cell and decrease toxicity to healthy cells. Unlike 4-N-alkanoyl gemcitabine analogues, whose hydrolyzable modifications has been shown to slowly release the parent drug in vivo, 4- $\mathrm{N}$-alkyl modified analogues of gemcitabine lack hydrolysis of the side chain in the cell. The lack of hydrolysis prevents enzymatic deamination of gemcitabine and makes these analogues useful for addition of radiolabels that allow for PET imaging. ${ }^{33}$

The synthesis of 4- $N$-alkyl gemcitabine bearing a $\beta$-keto tosylate moiety $\mathbf{5 6}$ involves the addition of an 11-carbon alkyl chain bearing a terminal alkene. The elaboration of the 
terminal alkene to $\beta$-keto tosylate involves dihydroxylation, tosylation and oxidation. The analogue 56 should provide efficient ${ }^{18} \mathrm{~F}$ - fluorination given the reactivity of the $\beta$-keto tosylate and avoid defluorination, seen in previous analogues, ${ }^{33}$ as a result of the lack of hydrogens at $\beta$-carbon. The synthesis of 4-N-alkyl-NOTA-gemcitabine chelator $\mathbf{6 5}$ begins by $4-\mathrm{N}$-alkylation of gemcitabine followed by condensation with the SCN-Bn-NOTA chelator. The procedure should provide a novel analogue useful for chelation of ${ }^{68} \mathrm{Ga}$ for use as a PET radiotracer.

Because of our experience with synthesis of ${ }^{18} \mathrm{~F}$-radiolabelled fluoro-nucleoside analogues, the second aim of the dissertation is the synthesis of clofarabine 5'-diphosphate ProTides (e.g., I; Figure 30) and potentially their ${ }^{18}$ F-radiolabelled analogues II. ${ }^{65}$ These analogues can be used to bypass the rate-limiting phosphorylation steps that lower their efficacy. To synthesize clofarabine 5'-diphosphate Pro-Tides, certain specification should be taken into consideration: 1) $\alpha$-phosphate should contain no modification because negative charge on oxygen avoids release of clofarabine monophosphate by cleavage of the phosphorous anhydride bond, and 2) $\beta$-phosphate should contain lipophilic modifications that allow uptake of ProTide into the cell and can be metabolized to release unmasked clofarabine 5'-diphosphate. The synthesis of these ProTides involves synthesis of clofarabine 5'-monophosphate, followed by coupling to a reactive phosphoramidite species. Addition of ${ }^{18} \mathrm{~F}$-radiolabel can be attempted using methods similar to those used for synthesis of ${ }^{18} \mathrm{~F}$-clofarabine. ${ }^{202}$ 


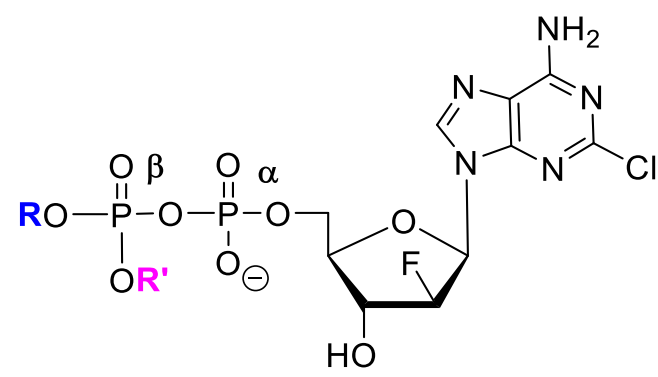

I

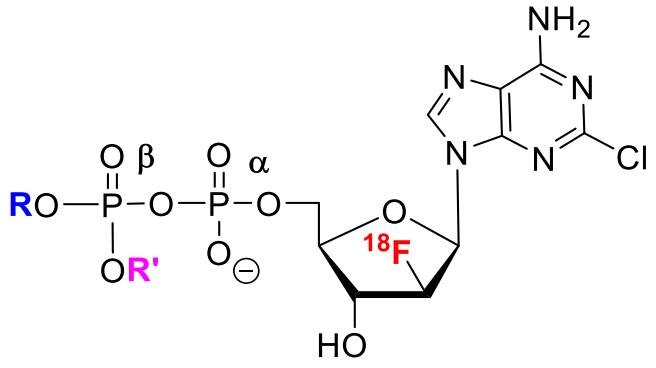

II

Figure 30. Proposed structure of clofarabine 5'-diphosphate ProTides

The final aim of this dissertation is to synthesize several azido-modified purine and pyrimidine nucleosides (e.g., III-VI; Figure 31) for the study of aminyl radical formation during addition of radiation-produced electrons. The azide functionality is known to have a very high electron affinity, which has been utilized for its radiosensitizing potential in drugs such as $3^{\prime}$-AZT for the treatment of cancer in HIV patients ${ }^{104}$ and recently reported by our research lab for AmdU. ${ }^{106}$ The radical responsible for the radiosensitizing effect of these analogs is the nitrogen centered aminyl radical $(\bullet \mathrm{NH})$, which is formed when an azide captures an electron. The aminyl radical can then interact with different DNA bases, ultimately causing DNA breakage and cell death. I propose, then, that synthesis of three classes of nucleosides modified with azide group directly attached to the base moiety should allow characterization of the aminyl radical by EPR. The first class of compounds are 2-azidoinosine analogues (e.g. IV), which could be prepared by deamination of 2azidoadenosine analogues (e.g. III), for elucidation of the elusive guanyl aminyl radical. The second class of compounds includes 6-azidoadenosine analogue (e.g. VI) that can be used to characterize the adenyl aminyl radical. The final class are 4-azidouridine analogues, which can be used to elucidate the cytidyl aminyl radical. 


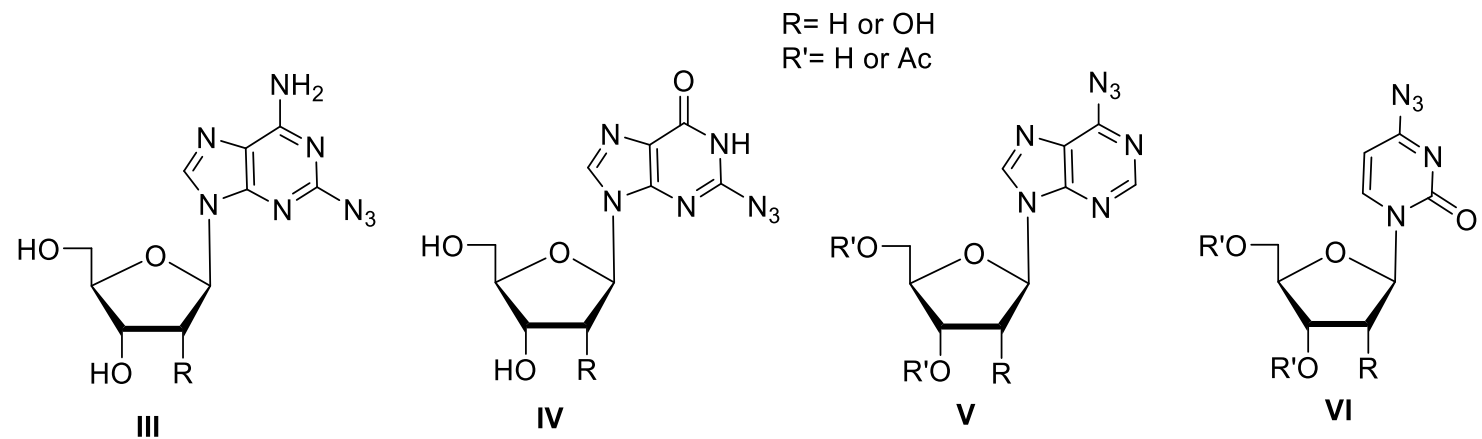

Figure 31. Structure of proposed azido modified nucleosides for study of aminyl radical

Since, as discussed in introduction (Sec. 1.4.5), it is known that base-modified azido nucleosides exist in equilibrium with the corresponding tetrazoles (e.g., VII and VIII, Figure 32). Investigating reactivity and chemistry of the tetrazole derivatives upon addition of radiation-produced electrons is of much interest as well. The tetrazole analogues are expected to produce the same aminyl radical as their azido counterparts, thus producing the same characteristic EPR spectra. Study of the aminyl radical formation from tetrazole derivatives is especially important since these derivatives can be incorporated onto DNA by solid-phase synthesis ${ }^{199}$, unlike their unstable azido counterparts. If EPR studies are successful, biological studies of the tetrazole analogues will be done in order to test for anti-cancer activity in vivo.

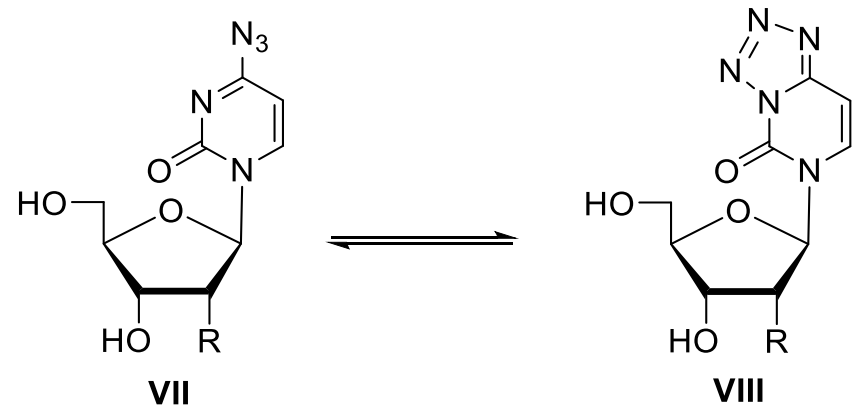

Figure 32. Azido-Tetrazole equilibrium in 4-azidopyrimidine nucleosides 


\section{RESULTS AND DISCUSSION}

\subsection{Synthesis of 4- $N$-alkyl gemcitabine analogues}

\subsubsection{4- $N$-Alkylgemcitabine with $\beta$-keto tosylate moiety}

Our first target was a 4- $N$-alkyl gemcitabine analogue bearing a $\beta$-keto tosylate moiety for subsequent ${ }^{18}$ F-radiolabeling for PET imaging (e.g., 56; Figure 33). The addition of an 11-carbon alkyl chain to the $4 N$ position of gemcitabine was chosen as a means to increase lipophilicity for increased cellular uptake, ${ }^{27,203}$ as well as, to avoid deamination by dCDA. ${ }^{32}$ The lack of hydrolysis exhibited by $4-N$-alkyl modifications on the cytosine base causes a low amount of phosphorylation and DNA incorporation, but allows for chemical modifications, such as, addition of a radiolabel $\left({ }^{18} \mathrm{~F}\right) .{ }^{33}$ The $\beta$-keto tosylate moiety not only activates the $\alpha$-carbon, and therefore, facilitates fluorination, but also avoids possible in vivo defluorination due to the lack of hydrogens in the $\beta$-carbon.

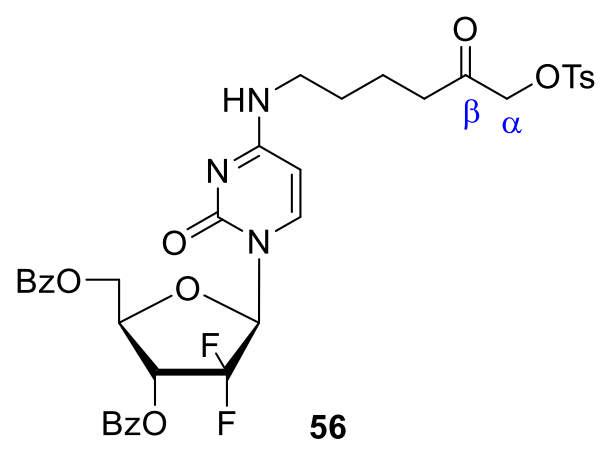

Figure 33. 4- $N$-Alkylgemcitabine derivative bearing $\beta$-keto tosylate moiety for subsequent fluorination

Our lab has already reported 4- $N$-acyl and 4- $\mathrm{N}$-alkyl gemcitabine analogues with terminal leaving groups, which can be used for ${ }^{18} \mathrm{~F}$-radiolabeling (Figure 34). However, the 4- $\mathrm{N}$-acyl analogue IX showed cleavage of the amide linkage under fluorination conditions used in ${ }^{18} \mathrm{~F}$ labeling $\left(\mathrm{KF} / \mathrm{K}_{2} \mathrm{CO}_{3} / \mathrm{K}_{2.2 .2} / \mathrm{CH}_{3} \mathrm{CN} / 110{ }^{\circ} \mathrm{C}\right) .{ }^{33,204} \mathrm{On}$ the other hand, we found that incorporation of ${ }^{18} \mathrm{~F}$-radiolabel on $4-N$-alkylgemcitabine analogue $\mathbf{X I}$ produced 
fluorinated XII successfully, yet showed loss of ${ }^{18} \mathrm{~F}$ during in vivo mice studies. ${ }^{204}$ In order to overcome these drawbacks, our lab also synthesized $4-N$-alkyl gemcitabine analogues with a silicon-fluoride acceptors moieties that have shown to be good substrates for ${ }^{18} \mathrm{~F}$ labeling (e.g., XIII $\rightarrow$ XIV). ${ }^{205}$

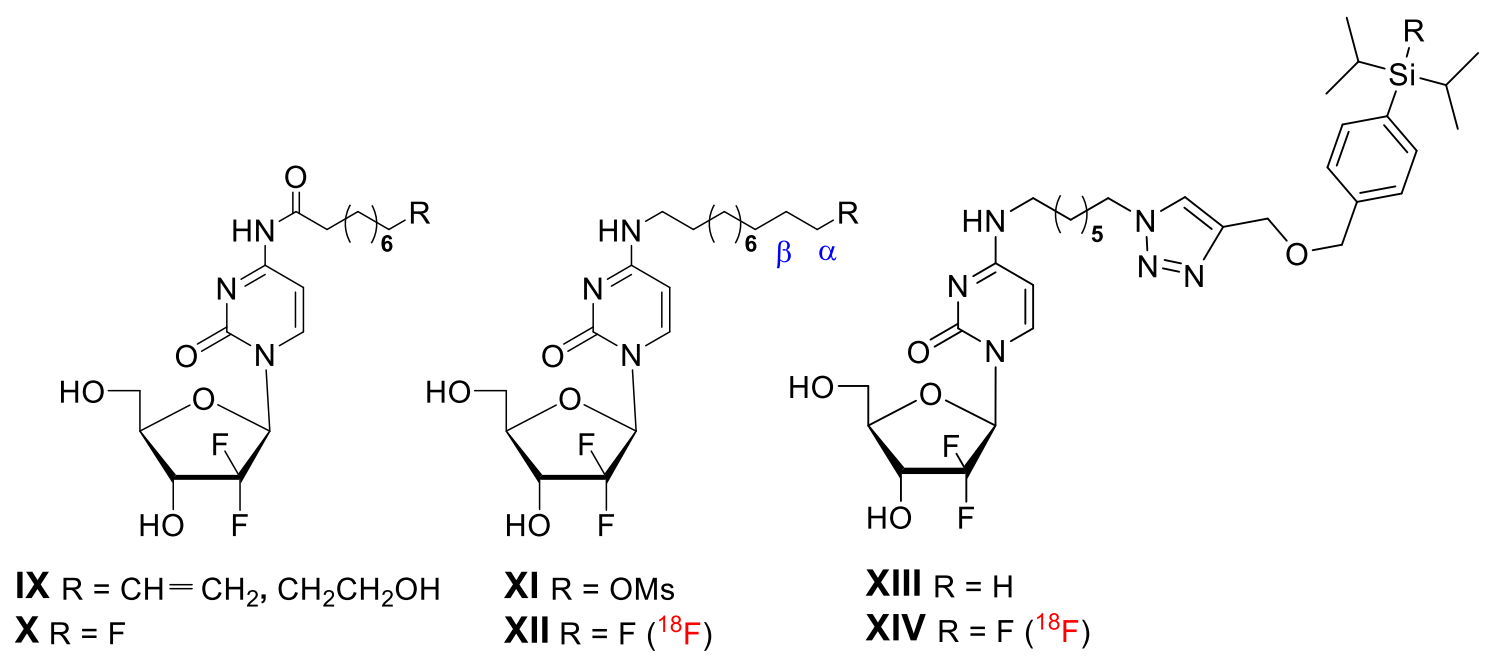

Figure 34. Gemcitabine analogues suitable for ${ }^{18} \mathrm{~F}$-radiolabelling

\subsubsection{4-N-Alkyl $\beta$-keto tosylate gemcitabine analogue: Synthesis}

The main steps in synthesis of $4-N$-alkyl gemcitabine derivatives suitable for fluorination involves displacement of 4- $\mathrm{N}$-tosylamine group from gemcitabine with commercially available 1-amino-10-undecene, followed by elaboration of the terminal olefin into $\beta$-keto tosylate precursor (e.g., 56; Figure 33). Thus, synthesis of $\mathbf{5 6}$ began by alkylation of gemcitabine $\mathbf{5 7}$ through tosylation of the 4-exocyclic amine with $\mathrm{TsCl}$, using transient protection with TMS-Cl/pyridine and subsequent deprotection in the presence of $\mathrm{MeOH} / \mathrm{NH}_{3}$, to afforded 4- $N$-tosylgemcitabine 58 (90\%; Scheme 18). ${ }^{33}$ Then, treatment of 58 with 1-amino-10-undecene and $\mathrm{Et}_{3} \mathrm{~N}$ in 1,4-dioxane effected displacement of the $p$ toluenesulfonamido group from the $\mathrm{C} 4$ position of the cytosine ring to give $4-\mathrm{N}-(10-$ undecenyl) derivative $\mathbf{5 9}(85 \%){ }^{33}$ 


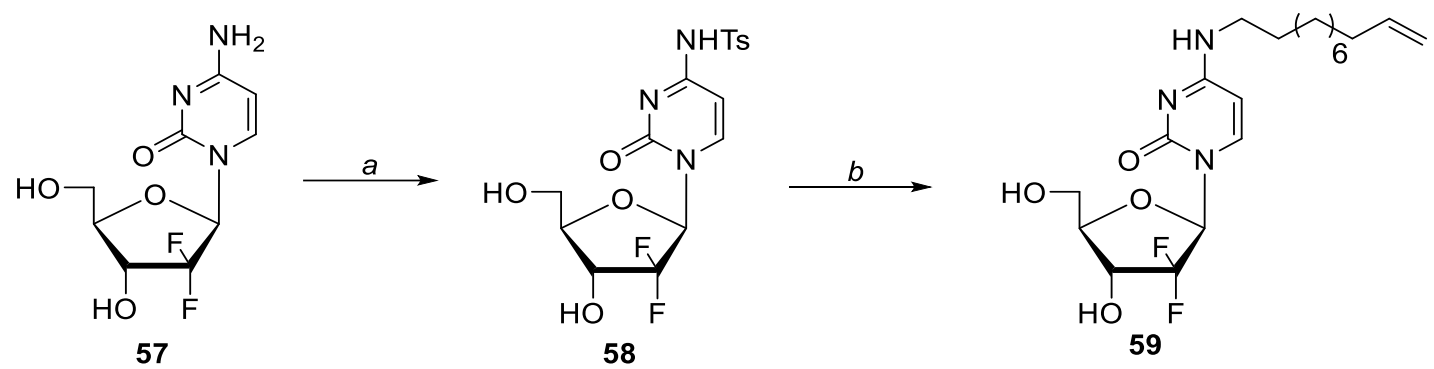

Reagents and Conditions: (a) (i) $\mathrm{TMSCl} / \mathrm{Pyr}$, (ii) $\mathrm{TsCl}$, (iii) $\mathrm{MeOH} / \mathrm{NH}_{3}$;

(b) 1-amino-10-undecene/ $\mathrm{Et}_{3} \mathrm{~N} / 1,4$-dioxane.

Scheme 18. Synthesis of $4-N$-alkylgemcitabine with terminal alkene

The terminal alkene was then elaborated through a series of steps to $\beta$-keto tosylate functionality. Synthesis began with benzoylation of $\mathbf{5 9}$ using benzoyl chloride $(\mathrm{BzCl})$, dimethylamino pyridine (DMAP), and 2,6-lutidine to yield 3' and 5' protected 4- $\mathrm{N}$-alkyl gemcitabine $\mathbf{6 0}(86 \%$, Scheme 19). This was followed by dihydroxylation of $\mathbf{6 0}$ with catalytic $\mathrm{OsO}_{4}$ in the presence of $N$-methylmorpholine $N$-oxide (NMO) produced terminal vicinal diol 61 (96\%). Terminal vicinal diol $\mathbf{6 1}$ was then converted to $\beta$-keto tosylate $\mathbf{5 6}$ by regioselective tosylation of the primary hydroxyl followed by oxidation of the secondary hydroxyl. Thus, treatment of $\mathbf{6 1}$ with tosyl chloride $(\mathrm{TsCl})$ gave regioselectively tosylate $62(62 \%)$, along with unchanged 61 (20\%), but no notable formation of ditosylated product. Tosylate 62 was found to be somewhat unstable, decomposing in a few months, even when kept in dry conditions in refrigerator. Oxidation of tosylate $\mathbf{6 2}$ with Collins reagent $\left(\mathrm{CrO}_{3}\right.$, pyridine and $\mathrm{Ac}_{2} \mathrm{O}$ ) gave $\beta$-keto tosylate 56 (94\%) as a precursor for fluorination. Compound 56 was relatively unstable, lasting only a few weeks when kept solid in the refrigerator. This instability is thought to be due to the high reactivity of the $\alpha$-carbon in carbonyls. $^{206}$ 


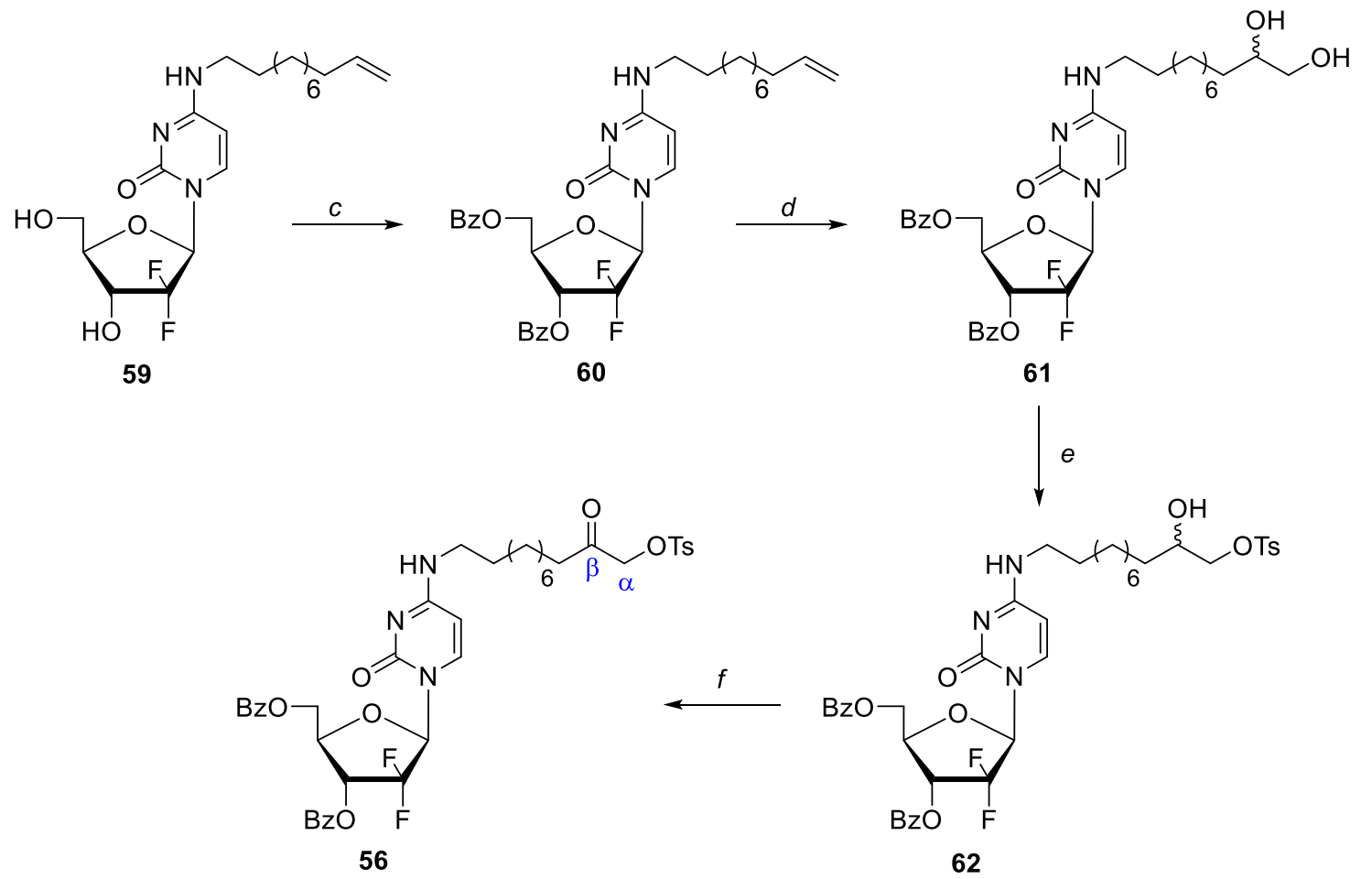

Reagents and Conditions: (c) $\mathrm{BzCl} / \mathrm{DMAP} / 2$,6-lutidine/DCM; (d) $\mathrm{OsO}_{4} / \mathrm{NMO} /$ acetone $/ \mathrm{H}_{2} \mathrm{O}$ (10:1);

(e) $\mathrm{TsCl} / \mathrm{Et}_{3} \mathrm{~N} / \mathrm{DCM}$; (f) Collin's Reagent $\left(\mathrm{CrO}_{3} / \mathrm{pyr} / \mathrm{Ac}_{2} \mathrm{O}\right)$.

Scheme 19. Synthesis of $4-N$-alkyl gemcitabine bearing $\beta$-keto tosylate moiety

\subsubsection{4- $N$-Alkyl $\beta$-keto tosylate gemcitabine analogue: Attempted}

\section{fluorination}

Treatment of the reactive $\beta$-keto tosylate $\mathbf{5 6}$ with $\mathrm{KF}$ in the presence of 18 -crown-6 in $\operatorname{MeCN}\left(65^{\circ} \mathrm{C}, 1 \mathrm{~h}\right)$ gave fluorinated $\mathbf{6 3}$ in $38 \%$ yield after column chromatography, in addition to unchanged starting material $(\sim 50 \%)$, giving a practical yield of $76 \%$ for the conversion of $\mathbf{5 6}$ to $\mathbf{6 3}$. It is important to note that $\beta$-keto tosylate 56 provided a clean fluorination with virtually no formation of side products. The three set of signals in ${ }^{19} \mathrm{~F}$ NMR were diagnostic for the presence of three fluorine atoms in 63 [-119.41 (d, $J=245.1$ $\mathrm{Hz}, 1 \mathrm{~F}$ ) and $-120.17 \mathrm{ppm}\left(\mathrm{br} \mathrm{d}, J=245.1 \mathrm{~Hz}, 1 \mathrm{~F}\right.$ ) for the $\mathrm{C}^{2}$ gem-difluoro unit and triplet at $-227.42(J=47.7,1 \mathrm{~F})$ for the terminal $\left.\mathrm{CH}_{2} \mathrm{~F}\right]$. Deprotection of $\mathbf{6 3}$ with $\mathrm{MeOH} / \mathrm{NH}_{3}(0$ ${ }^{\circ} \mathrm{C}$ to $\left.\mathrm{rt}, 2 \mathrm{~h}\right)$ gave $\alpha$-fluoromethyl ketone 64 (52\%, Scheme 20$) .{ }^{206}$ 

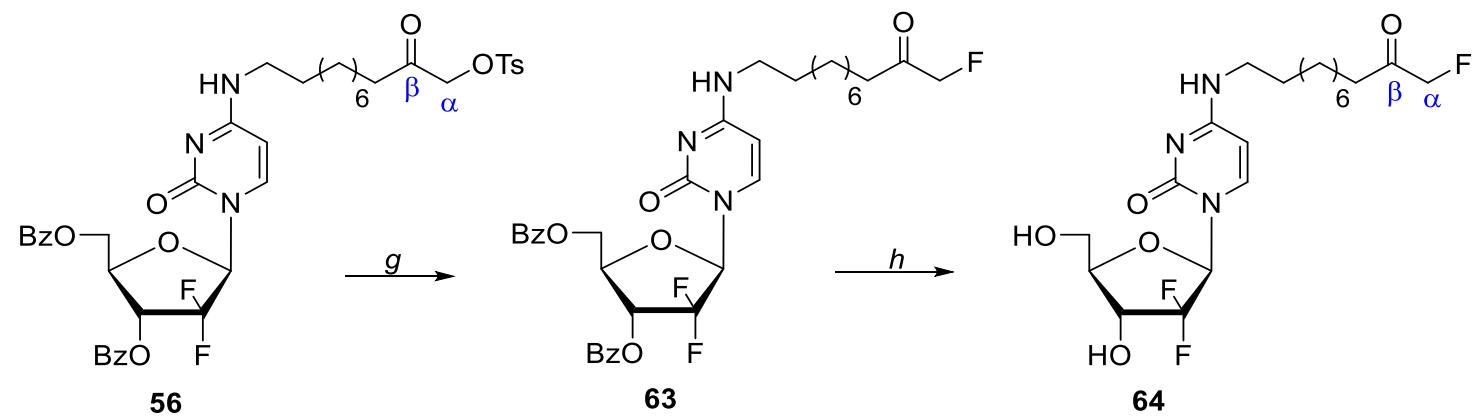

Reagents and Conditions: g) $\mathrm{KF} / 8-\mathrm{Crown}-6 / \mathrm{MeCN}$, or Kryptofix 2.2.2, $70{ }^{\circ} \mathrm{C}, 0.5-1 \mathrm{~h}$; h) $\mathrm{MeOH} / \mathrm{NH}_{3}, 0^{\circ} \mathrm{C}$ to $\mathrm{rt}, 30 \mathrm{~min}$ to $2 \mathrm{~h}$.

Scheme 20. Fluorination of $4-N$-alkyl $\beta$-keto tosylate gemcitabine analogue

Fluorination employing ${ }^{18}$ F-PET radiolabeling protocols was then attempted for $\mathbf{5 6}$. Therefore, treatment of $\mathbf{5 6}$ with $\mathrm{KF} / \mathrm{Kryptofix}$ 2.2.2. (75 $\left.{ }^{\circ} \mathrm{C}, \mathrm{MeCN}, 30 \mathrm{~min}\right)$, followed immediately by deprotection of the crude 63 with $\mathrm{MeOH} / \mathrm{NH}_{3}$ (r.t., $45 \mathrm{~min}$ ) gave 64 in low yield [ $\sim 5$ by Thin Layer Chromatography (TLC)] with formation of several byproducts. Separation of $\mathbf{6 4}$ from the side products required extensive HPLC purification, making this method unfeasible for the development of $\left[{ }^{18} \mathrm{~F}\right]-\mathbf{6 4}$ as a potential radiotracer. Most likely, during the deprotection of $\mathbf{6 3}$, ammonia reacts with unchanged $\beta$-keto sulfonate substrate 56 and/or with the reactive $\alpha$-fluoroketone moeity leading to a primary amine and/or the formation of imine byproducts. ${ }^{32}$

\subsubsection{4-N-Alkyl gemcitabine conjugated to NOTA ${ }^{68}$ Ga chelators}

The problematic fluorination by standard ${ }^{18} \mathrm{~F}$-radiolabeling protocols which we observed in the 4-N-alkylgemcitabine $\beta$-keto tosylate analogue 56, prompted us to search for other gemcitabine conjugates suitable for PET imaging. Recent advances in aza-crown ethers bifunctional chelators with the ability to bind gallium generated significant interest in ${ }^{68} \mathrm{Ga}$-based radiopharmaceuticals ${ }^{72-76}$ and radiotracers. ${ }^{76,77}$ Thus, we began to explore the idea of 4- $N$-alkylgemcitabine analogues linked to bifunctional gallium chelators. 


\subsubsection{4- $N$-Alkyl gemcitabine NOTA ${ }^{68}$ Ga chelators: Synthesis}

Since 4- $\mathrm{N}$-alkylgemcitabine derivatives are resistant to chemical hydrolysis as well as enzymatic deamination by $\mathrm{dCDA},{ }^{33}$ we began to explore such derivatives, which would allow for facilitated addition of a radioactive nuclei $\left({ }^{11} \mathrm{C},{ }^{18} \mathrm{~F},{ }^{64} \mathrm{Cu},{ }^{68} \mathrm{Ga}\right.$, etc.). Given recent literature of ${ }^{68} \mathrm{Ga}$ based radiopharmaceuticals, with the facile condensation of commercially available SCN-Bn-NOTA to the N-terminal of amino acids ${ }^{77}$ and highly accommodating coordination cavity of the NOTA moiety to gallium, ${ }^{81,82}$ we undertook an effort to prepare gemcitabine-NOTA ligand conjugated via 4-N-alkyl linker (65, Figure $35) .{ }^{207}$

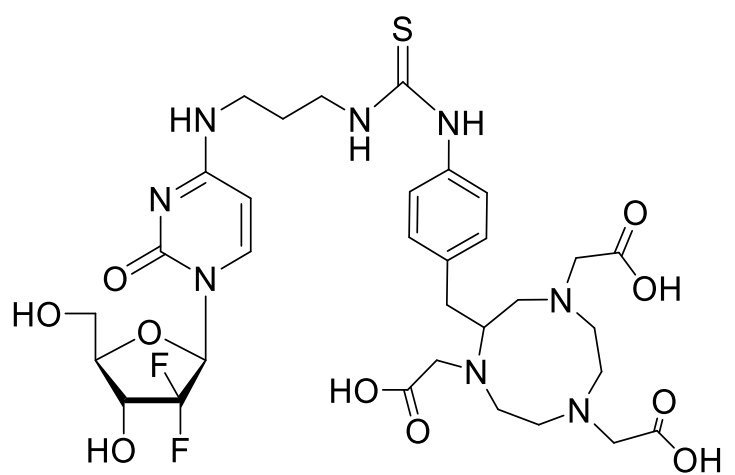

65

Figure 35. 4- $N$-alkylgemcitabine-NOTA analogue for ${ }^{68} \mathrm{Ga}$-radiolabeling

Similarly to previously synthesized $4-N$-alkyl gemcitabine analogues, synthesis began with displacement of $p$-toluenesulfonamido in protected 4 - $N$-tosylgemcitabine 58a (prepared from gemcitabine in $96 \%$ yield) ${ }^{33}$ with commercially available $N$-Boc-1,3propanediamine in 1,4-dioxane affording as main product the partially deprotected 4- $\mathrm{N}$-(3$N^{\prime}$-Boc-3-aminopropanyl)gemcitabine $\mathbf{6 6}(59 \%$, Scheme 21) in addition 3',5'-di- $O$-Boc protected product 66a $(31 \%)$. Subsequent deprotection of 66 with TFA yielded the 4- $N$-(3aminopropanyl) derivative $\mathbf{6 7}$ (93\%), with a primary amine group suitable for condensation with NOTA ligands. Alternatively, compound $\mathbf{6 7}$ was prepared efficiently in a "one-pot" 
synthesis from $\mathbf{5 8}$ without isolation of $\mathbf{6 6}$. This method involved the reaction of $\mathbf{5 8}$ with $\mathrm{N}$ Boc-1,3-propanediamine and $\mathrm{Et}_{3} \mathrm{~N}$ in 1,4 -dioxane at $75^{\circ} \mathrm{C}$, followed by immediate treatment with trifluoroacetic acid (TFA) to afford $94 \%$ of $\mathbf{6 7} .{ }^{207}$

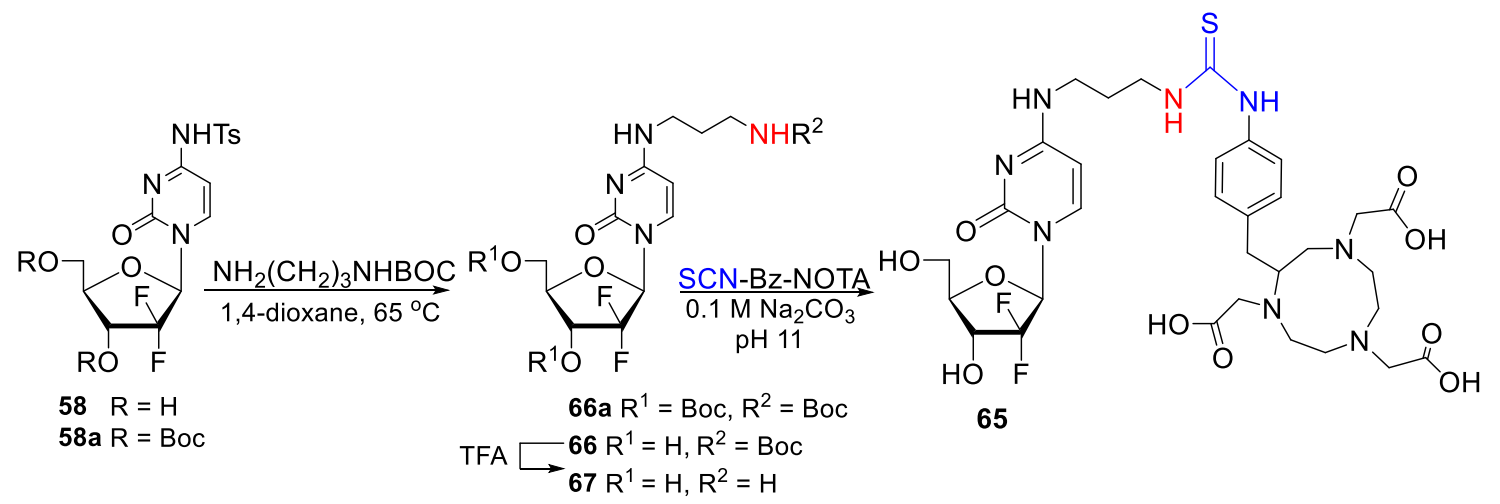

Scheme 21. Preparation of the gemcitabine-NOTA conjugate with $4-N$-alkyl linker ${ }^{207}$

Condensation of 67 with commercially available SCN-Bn-NOTA in $0.1 \mathrm{M} \mathrm{Na}_{2} \mathrm{CO}_{3}$ buffer $(\mathrm{pH}=11)$ at ambient temperature for $48-72 \mathrm{~h}$ afforded the $4-N-(3-\mathrm{SCN}-\mathrm{Bn}-\mathrm{NOTA}$ propanyl)gemcitabine conjugate $\mathbf{6 5}$ in $54 \%$ yield, as identified by HRMS $\left(\mathrm{ESI}^{+}\right) \mathrm{m} / \mathrm{z}$ 771.2879. The 4- $N$-alkylgemcitabine-NOTA conjugate $\mathbf{6 5}$ was found to be stable as a solid, however some minor deterioration back to 67 (TLC/HPLC) was observed after some time (few weeks) even when stored at $-20{ }^{\circ} \mathrm{C}$ (Figures 36-38). This showed again that gemcitabine analogues with $4-N$-alkyl chains have better stability that the corresponding 4- $N$-acyl counterparts, which usually decomposed during condensation reactions with NOTA analogues. ${ }^{207}$ 


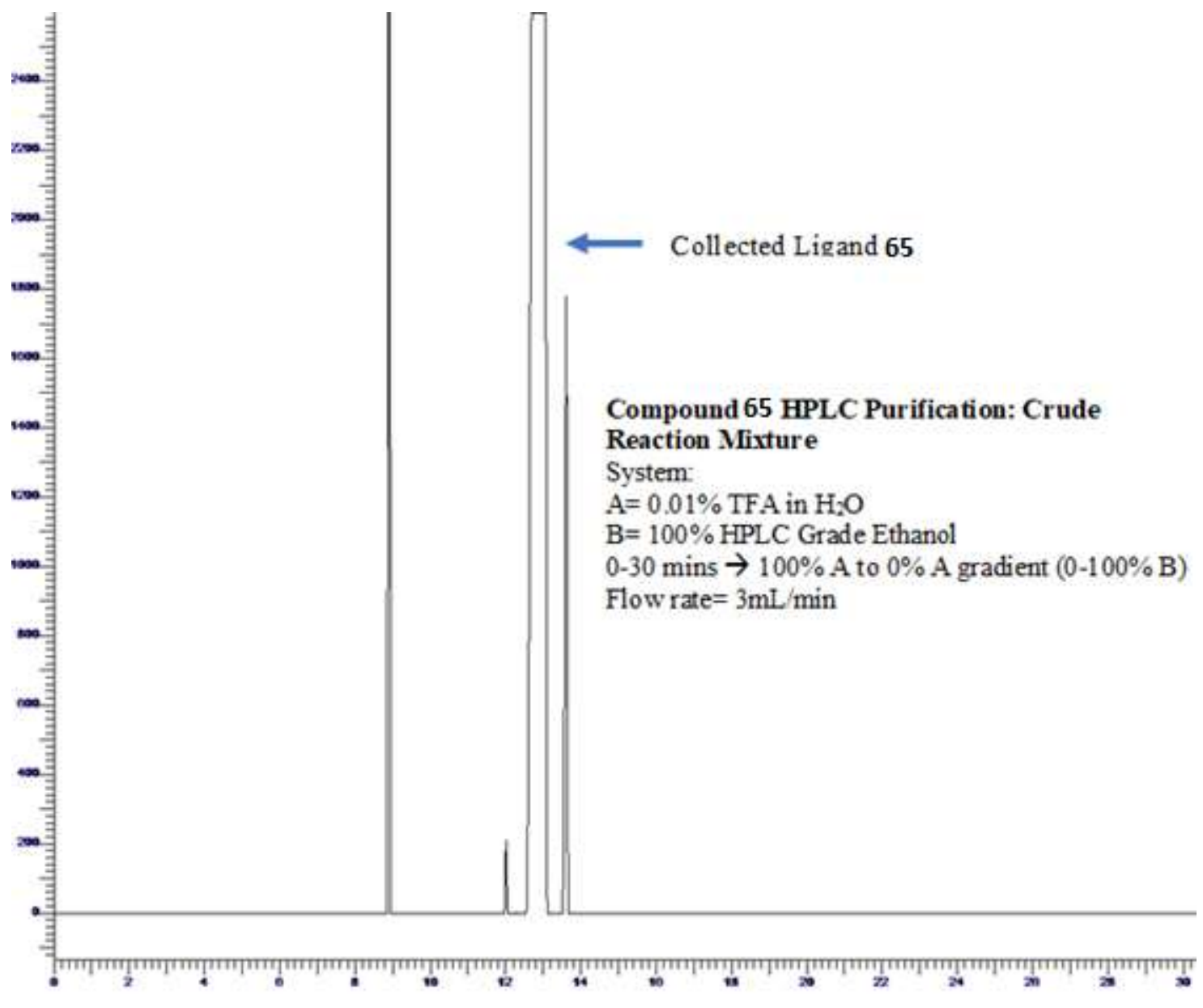

Figure 36. HPLC purification of conjugate 65 from the crude reaction mixture 


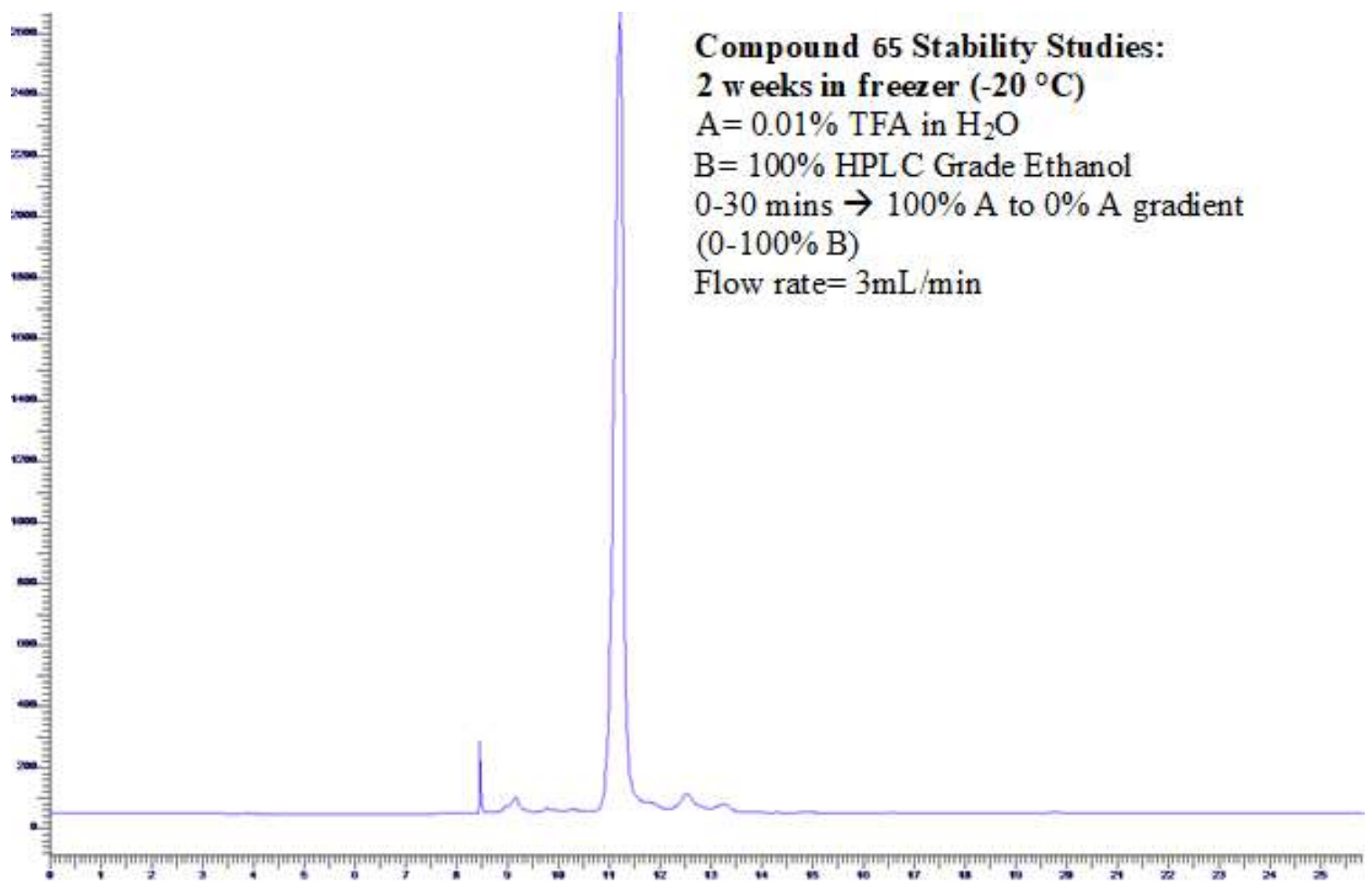

Figure 37. Stability studies of compound 65 after 2 weeks in a $-20^{\circ} \mathrm{C}$ fridge

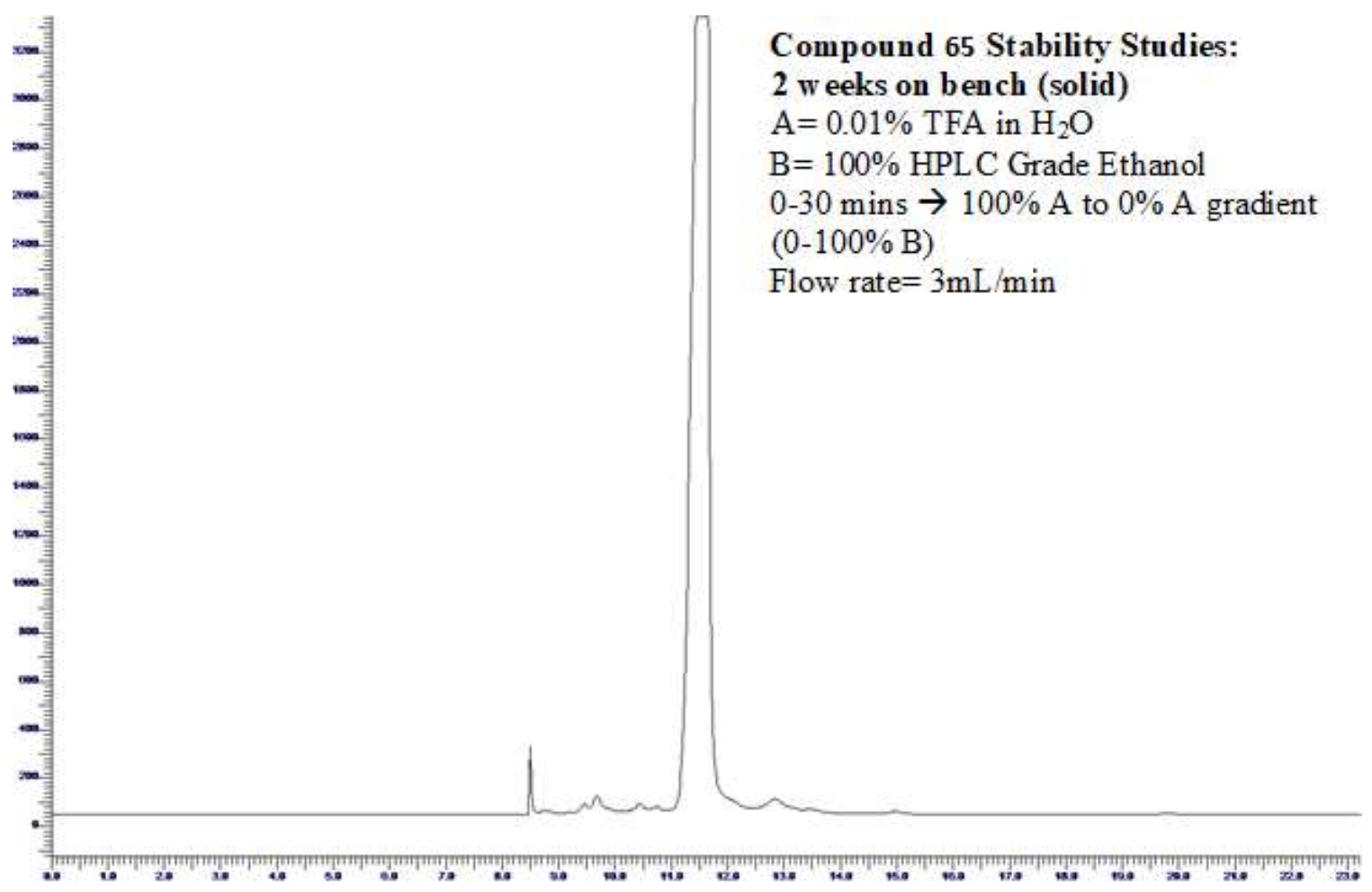

Figure 38. Stability studies of solid $\mathbf{6 5}$ after 2 weeks on bench 
Incubation of $\mathbf{6 5}$ with excess of gallium(III) chloride $\left(\mathrm{GaCl}_{3}\right)$ mimicking radiosynthetic conditions $\left(0.6 \mathrm{~N} \mathrm{AcONa} / \mathrm{H}_{2} \mathrm{O}, \mathrm{pH}=9.3\right)$ over 15 min gave the Gallium NOTA-4- $N$ alkylgemctiabine complex 68, characterized by HRMS (ESI+) m/z 837.1977 (Scheme 22). The reaction products were isolated by HPLC on a Phenomenex Gemini semi-preparative RP-C18 column $\left[0 \% \rightarrow 100 \%\right.$ ethanol gradient in $0.01 \% \mathrm{TFA} / \mathrm{H}_{2} \mathrm{O}$ from 0 to $30 \mathrm{~min}$ at a flow rate $=5 \mathrm{~mL} / \mathrm{min}]$ giving $\mathbf{6 5}\left(t_{\mathrm{R}}=14.1 \mathrm{~min}\right)$ and $\mathbf{6 8}\left(t_{\mathrm{R}}=12.4 \mathrm{~min}\right)$, respectively. The gallium complexation met criteria for working with $\left.\left[{ }^{68} \mathrm{Ga}\right]\right]^{3+}$ (half-life 68 min.) and was applied towards the radiosynthesis of the $\left[{ }^{68} \mathrm{Ga}\right]-\mathbf{6 8}$ radioligand.

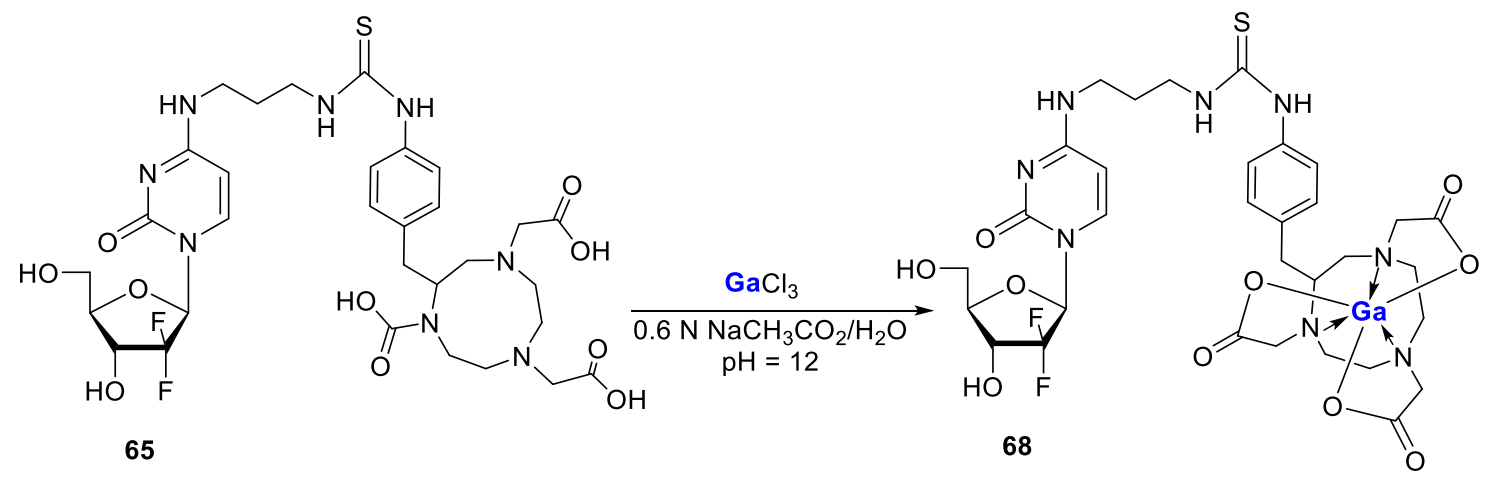

Scheme 22. Labeling of 4- $N$-alkylgemcitabine-NOTA conjugate with Gallium

\subsubsection{4- $N$-Alkyl gemcitabine NOTA ${ }^{68}$ Ga chelators: Labeling and Imaging}

Following success with the chelation of gallium to $4-\mathrm{N}$-alkylgemctiabine-NOTA chelator, we explored ${ }^{68} \mathrm{Ga}$-radiolabeling. The ${ }^{68} \mathrm{Ga}$ was eluted from an IGG- $100{ }^{68} \mathrm{Ge} /{ }^{68} \mathrm{Ga}$ generator. Purified $\left.\left[{ }^{68} \mathrm{Ga}\right]\right]^{+3}(10 \mathrm{mCi}$ in $1 \mathrm{~mL}$ of $\approx 0.1 \mathrm{~N} \mathrm{HCl})$ for the labeling was obtained following previously described conditions. ${ }^{208}$ 


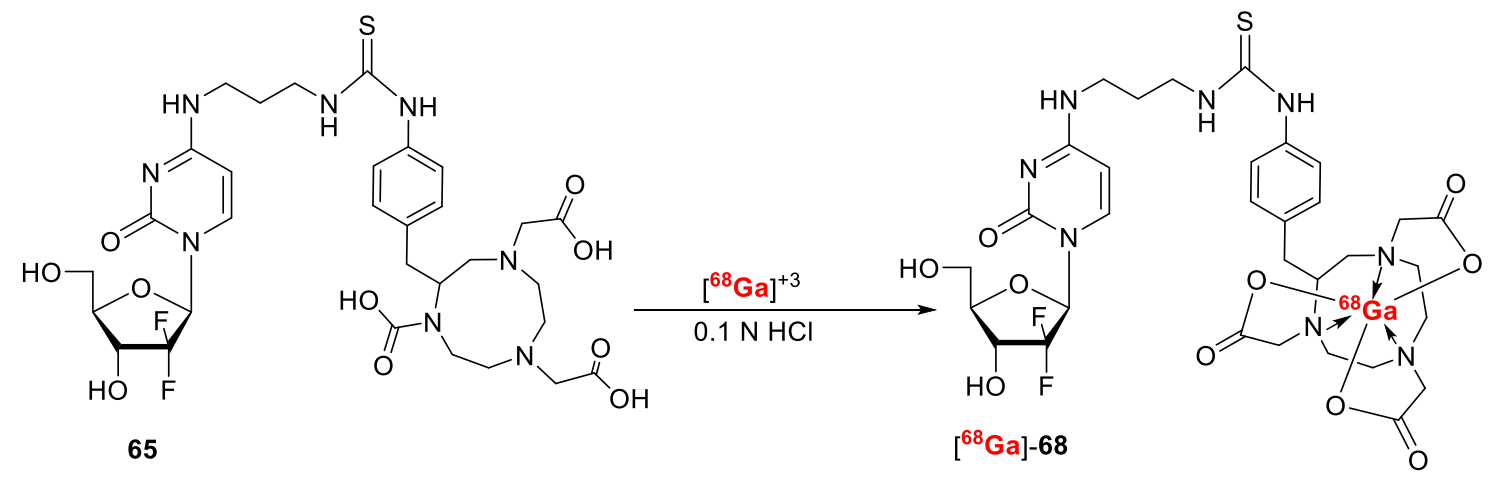

Scheme 23. Labeling of 4- $N$-alkylgemcitabine-NOTA conjugate with ${ }^{68} \mathrm{Ga}$

The $\left[{ }^{68} \mathrm{Ga}\right]$-labeling of $\mathbf{6 5}$ was completed within $15 \mathrm{~min}$ and analyzed by developing TLC plates eluted with 0.1 M citric acid on a phosphoscreen (Figure 39). Phosphoscreen analysis (Table 6) indicated a high labeling efficiency (94\% to 96\%) for the complexation of the NOTA-4- $N$-alkylgemcitabine 65 with $\left[{ }^{68} \mathrm{Ga}\right]{ }^{3+}$ to yield $\left[{ }^{68} \mathrm{Ga}\right]-\mathbf{6 8} .{ }^{207}$ The distribution coefficient of $\left[{ }^{68} \mathrm{Ga}\right]$-radioligand $\mathbf{6 8}$ was evaluated in octanol/ $\mathrm{H}_{2} \mathrm{O}$ and EtOAc/ $/ \mathrm{H}_{2} \mathrm{O}$. This ligand was almost exclusively found in the aqueous layer, with less $<5 \%$ of the observed counts occurring in the organic phase of either system. The hydrophilic character of $\left[{ }^{68} \mathrm{Ga}\right]-$ radioligand 68 suggested that the radioligand would be rapidly excreted. ${ }^{207}$

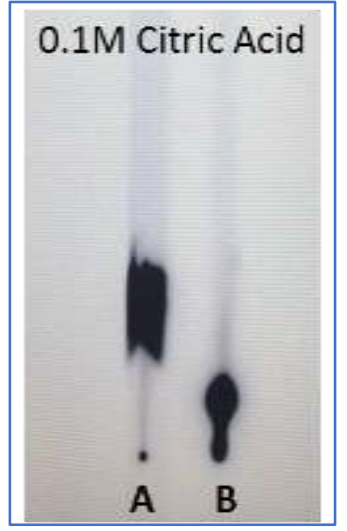

Figure 39. TLC for complexation of NOTA-4- $N$-alkylgemcitabine 65 with ${ }^{68} \mathrm{Ga}$. Free $\left[{ }^{68} \mathrm{Ga}\right]{ }^{3+}$ (lane A) and $\left[{ }^{68} \mathrm{Ga}\right]$-complexed-68 (lane B) 
Table 6. Labeling values for $4-N$-alkylgemcitabine-NOTA ligand 9 with ${ }^{68} \mathrm{Ga}$

\begin{tabular}{|c|c|c|c|c|c|c|}
\hline & \multicolumn{2}{|c|}{ Trial 1 } & \multicolumn{2}{c|}{ Trial 2 } & \multicolumn{2}{c|}{ Trial 3 } \\
\hline & $\begin{array}{c}\text { Control } \\
\left(\text { Free }{ }^{68} \mathrm{Ga}\right)\end{array}$ & $\begin{array}{c}\mathrm{Cmpd} \\
{\left[{ }^{68} \mathrm{Ga}\right]-14}\end{array}$ & $\begin{array}{c}\text { Control } \\
\left(\text { Free }{ }^{68} \mathrm{Ga}\right)\end{array}$ & $\begin{array}{c}\text { Cmpd } \\
{\left[{ }^{68} \mathrm{Ga}\right]-14}\end{array}$ & $\begin{array}{c}\text { Control } \\
\left(\text { Free }^{68} \mathrm{Ga}\right)\end{array}$ & $\begin{array}{c}\text { Cmpd } \\
{\left[{ }^{68} \mathrm{Ga}\right]-14}\end{array}$ \\
\hline Counts organic & 363069018 & 25232330 & 240306449 & 10064331 & 219716549 & 6458287 \\
\hline Counts aqueous & 7970885 & 374787348 & 4208914 & 256461215 & 3319270 & 166566169 \\
\hline Labeling Yield & $\approx 2 \%$ Appl. & $94 \%$ & $\begin{array}{c}\approx 2 \% \text { Appl. } \\
\text { Point }\end{array}$ & $96 \%$ & $\begin{array}{c}\approx \% \text { Appl. } \\
\text { Point }\end{array}$ & $96 \%$ \\
& Point & & & & \\
\hline
\end{tabular}

Labeling of 65 with ${ }^{68} \mathrm{Ga}$ was repeated to test for distribution of $\left[{ }^{68} \mathrm{Ga}\right]-68$ in BALB/c mice. Chelation of ${ }^{68} \mathrm{Ga}$ to NOTA moiety in $\mathbf{6 5}$ was quantitative after 5 minutes incubation. However, a shoulder and some minor impurities peaks can be observed. The purity did not change after Sep-Pak purification, showing a shoulder (RT: $3.27 \mathrm{~min}$ ) and a minor peak (RT: $4.55 \mathrm{~min}$ ) that are still visible and quantifiable (Figure 40). However, these impurities were not expected to have a large effect on imaging studies. ${ }^{207}$

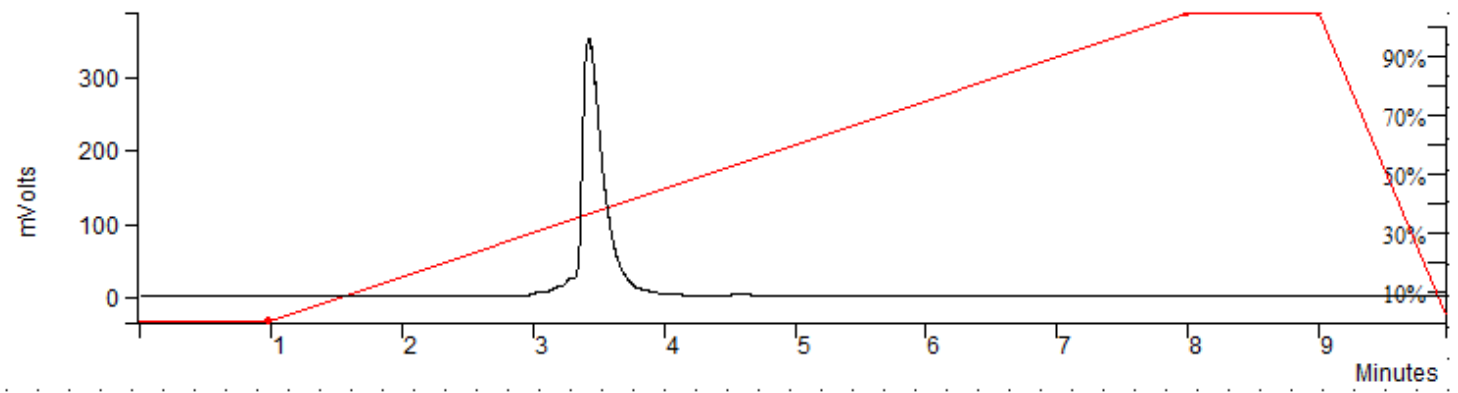

\begin{tabular}{|c|c|c|c|c|c|c|c|c|c|c|}
\hline $\begin{array}{c}\text { Peak } \\
\text { No }\end{array}$ & Peak Name & Result 0 & $\begin{array}{l}\text { Ret. } \\
\text { Time } \\
\text { (min) }\end{array}$ & $\begin{array}{l}\text { Time } \\
\text { Offset } \\
\text { (min) }\end{array}$ & $\begin{array}{c}\text { Area } \\
\text { (counts) }\end{array}$ & $\begin{array}{c}\text { Rel } \\
\text { Ret } \\
\text { Time }\end{array}$ & $\begin{array}{l}\text { Sep. } \\
\text { Code }\end{array}$ & $\begin{array}{c}\text { Width } \\
1 / 2 \\
(\mathrm{sec})\end{array}$ & $\begin{array}{l}\text { Status } \\
\text { Codes }\end{array}$ & Group \\
\hline 1 & & 0.0402 & 0.555 & 0.000 & 1665 & 0.00 & BB & 3.7 & & 0 \\
\hline 2 & & 0.6499 & 3.101 & 0.000 & 26908 & 0.00 & BV & 0.0 & & 0 \\
\hline 3 & & 2.8335 & 3.269 & 0.000 & 117321 & 0.00 & $\mathrm{vV}$ & 0.0 & & 0 \\
\hline 4 & & 95.9772 & 3.422 & 0.000 & 3973932 & 0.00 & VB & 9.0 & & 0 \\
\hline 5 & & 0.4588 & 4.552 & 0.000 & 18997 & 0.00 & $B B$ & 5.3 & & 0 \\
\hline 6 & & 0.0404 & 5.146 & 0.000 & 1671 & 0.00 & VP & 0.0 & & 0 \\
\hline & & 100.0000 & & 0.000 & 4140494 & & & & & \\
\hline
\end{tabular}

Figure 40. HPLC purification of $\left[{ }^{68} \mathrm{Ga}\right]-68$ from minor impurities after Sep-Pak purification 
Interestingly, in contrast with previously published studies with ${ }^{18} \mathrm{~F}-\mathrm{FAC},{ }^{62}$ compound $\left[{ }^{68} \mathrm{Ga}\right]-\mathbf{6 8}$ showed only minimal hepatobiliary excretion during in vivo mice studies. The rapid renal clearance of $\left[{ }^{68} \mathrm{Ga}\right]-\mathbf{6 8}$ is most likely due to the increased hydrophilicity caused by the addition of the NOTA chelator. Although not within the scope of the present work, this compound could result in higher contrast images due to higher lesion to background ratio, which could produce significant clinical impact. The one hour post-injection images are presented in Figure 41. ${ }^{207}$

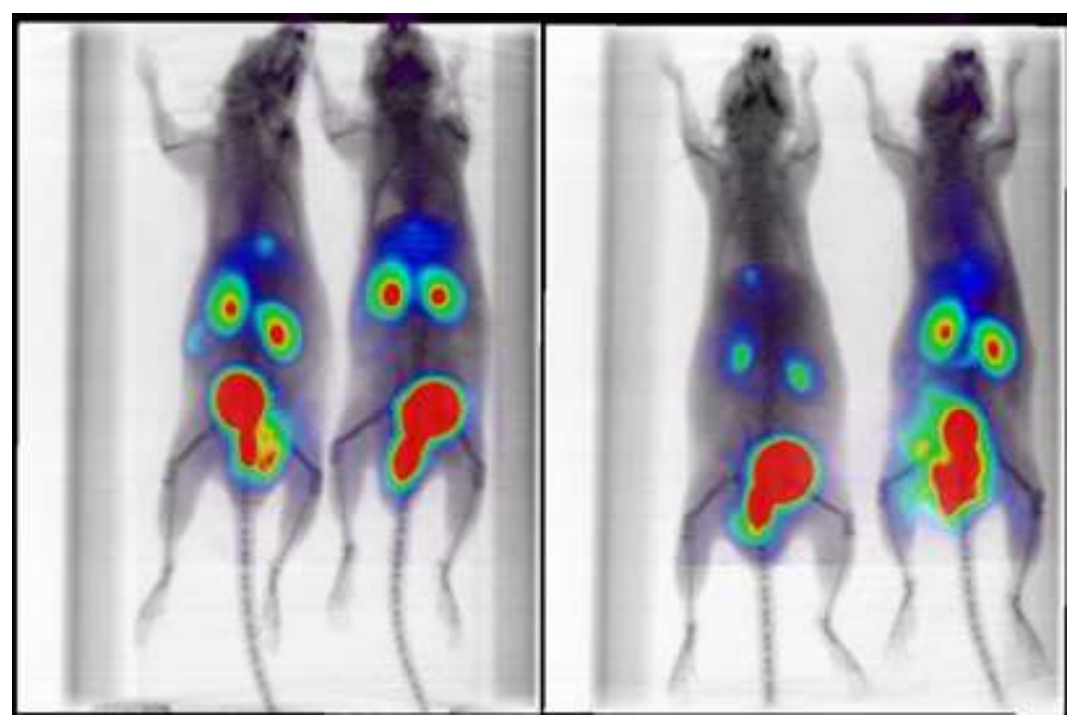

Figure 41. BALB/c maximum intensity projection (MIP) $\mu \mathrm{PET} / \mathrm{CT}$ mice images at onehour post injection showing rapid renal clearance

\subsection{Synthesis of Clofarabine 5'-diphosphate prodrugs}

\subsubsection{Clofarabine 5'-diphosphate prodrugs: significance and specifications}

Clofarabine, although highly effective, undergoes certain rate-limiting metabolisms in the cell. This includes rate-limiting phosphorylation from clofarabine 5'-monophosphate to clofarabine 5 '-diphosphate by purine nucleotide monophosphate kinase, ${ }^{15,20}$ unlike other drugs whose first phosphorylation event by dCK is usually rate-limiting. Clofarabine 5'monophosphate ProTides have been previously synthesized to bypass dCK metabolism. ${ }^{45}$ 
Little is known about the exact mechanism of 5'-nucleotidases; however, it has been found that there is an increased concentration of this enzyme in common acute lymphoblastic leukemia (ALL) cell lines causing dephosphorylation of adenosine monophosphate (AMP). ${ }^{22}$ This important because clofarabine is an adenosine analogue used to treat ALL, and possible clofarabine 5'-monophoshate ProTides experience dephosphorylation back to the parent drug in the cell. ${ }^{209}$ Thus, it is beneficial to synthesize diphosphate prodrug of clofarabine, which may overcome dephosphorylation by 5'nulceotidases and bypass rate-limiting phosphorylation.

In order to synthesize Clofarabine 5'-diphosphate produgs, certain specification should be taken into consideration based on recent literature findings:

- $\alpha$-phosphate should contain no modification because oxygen anion prevents nucleophilic attack that causes release of Clofarabine monophosphate by cleavage of the phosphorous anhydride bond. ${ }^{47}$

- $\beta$-phosphate should contain lipophilic modifications that allow uptake of ProTide into the cell and can be metabolized to release unmasked clofarabine 5'diphosphate. ${ }^{39}$
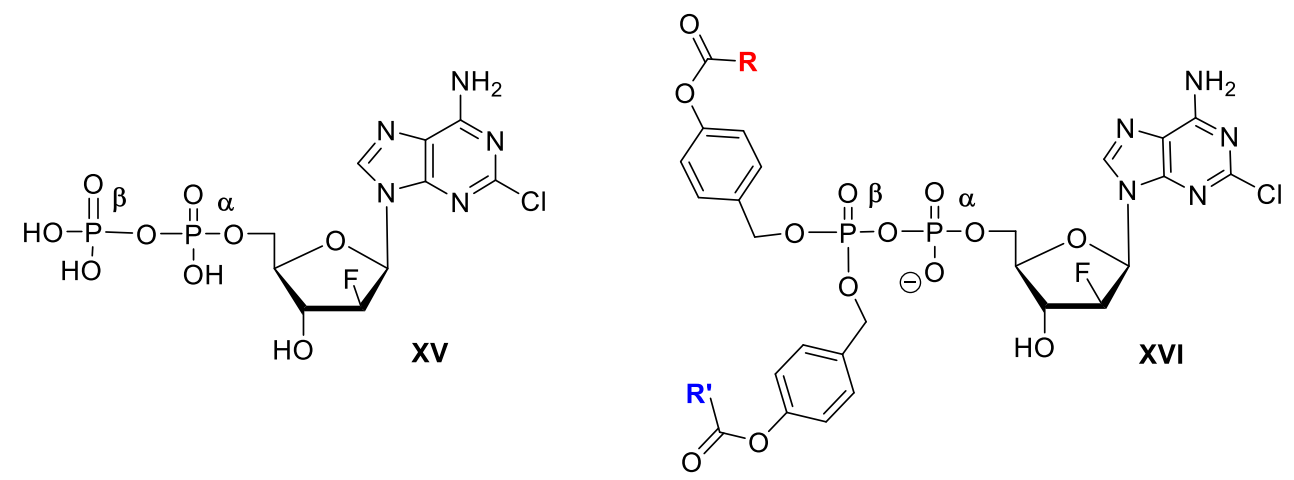

Figure 42. Clofarabine diphosphate and proposed Clofarabine diphosphate prodrugs. 
Because of recent literature by Dr. Meier's group based on the design and synthesis of diphosphate prodrugs (DiPPro) for use in anti-viral therapy, we decided to focus our efforts on synthesis of clofarabine diphosphosphate prodrugs of this type (e.g., XVI). Taking into account Meier's design of DiPPro, we divided the synthesis of our diphosphate analogues into three steps (Scheme 24).

The first step involved the synthesis of clofarabine using a variety of methods available in literature. ${ }^{15}$ The second step was synthesis of clofarabine 5'-monophosphate, by phosphorylation of the 5'-OH of clofarabine using Yoshikawa method. ${ }^{210}$ The third step was the coupling of clofarabine 5'-monophosphate to a bis(acyloxybenzyl) phosphoramidite. The results clofarabine 5'-diphosphate prodrug might be able to incorporate into the cell and release the unmasked clofarabine 5'-diphosphate by a mechanism as shown in Section 1.2 of this dissertation. ${ }^{52}$

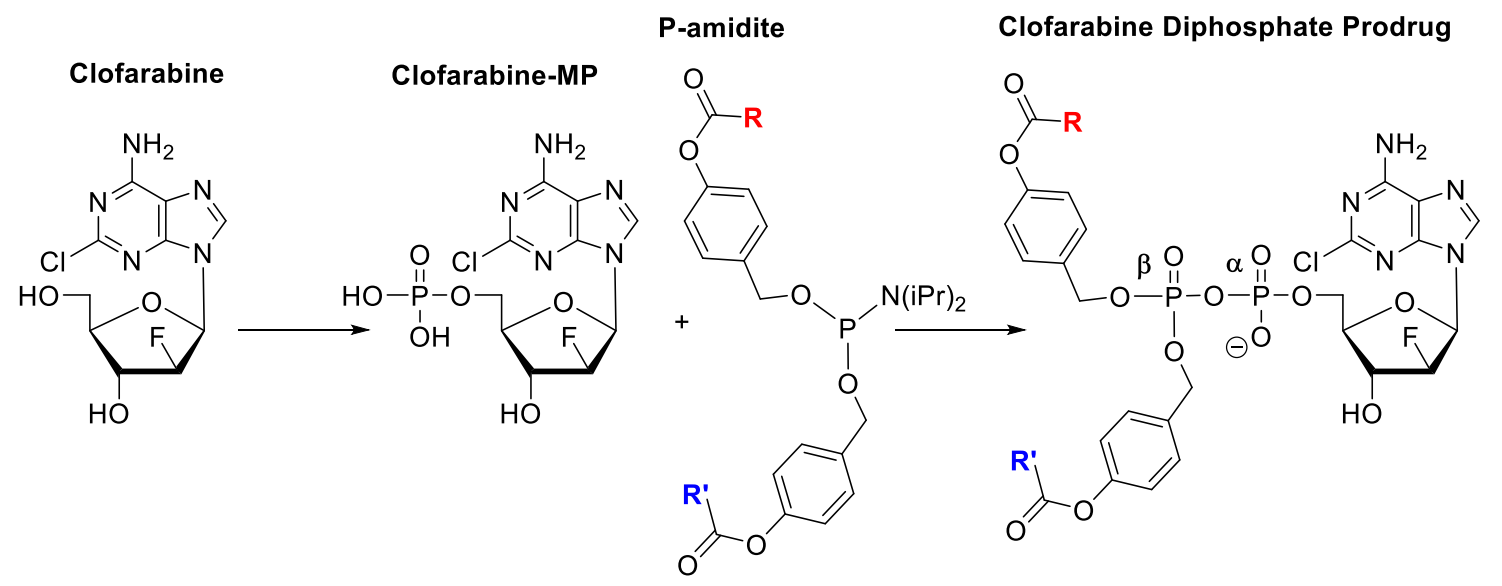

Scheme 24. Overall route to clofarabine 5'-diphosphate prodrugs

\subsubsection{Clofarabine}

We began by exploring available synthetic protocols to prepare the building blocks for our diphosphate prodrugs. Thus, synthesis of clofarabine was attempted using two 
methods; 1. using multi-step protocols to synthesize 2-chloroadenosine from guanosine (Schemes 25-26), ${ }^{21-213}$ followed by regioselective protection of 2-amino, and 3'- and 5' hydroxyl groups in order to perform substitution of the C2' hydroxy group with fluorine with inversion of stereochemistry using $\mathrm{DAST}^{214}$ (Scheme 27), or 2. using protocols to couple 2-deoxy-1- $\alpha$-bromo-2- $\beta$-fluoro-3,5-di-O-benzoyl-d-ribofuranose and 2chloroadenine units, followed by deprotection (Scheme 28). ${ }^{15,215}$

\subsubsection{Linear synthesis of clofarabine from guanosine}

Synthesis of clofarabine by method 1, began by acetylation of guanosine 69 with $\mathrm{Ac}_{2} \mathrm{O}$ /pyridine in DMF at $70{ }^{\circ} \mathrm{C}$ for 4 hours to afford 70 (88\%, Scheme 25). Then, phosphorylation to activate hydroxyl group at 6-position, followed by replacement with chlorine using $\mathrm{POCl}_{3} / \mathrm{Et}_{4} \mathrm{NCl} / \mathrm{N}, \mathrm{N}$-dimethylaniline in $\mathrm{ACN}$ at reflux for 30 mins gave 71 (70\%). Once compound $\mathbf{7 1}$ was made, the solubility in organic solvents was improved for further elaboration of the purine base..$^{211}$

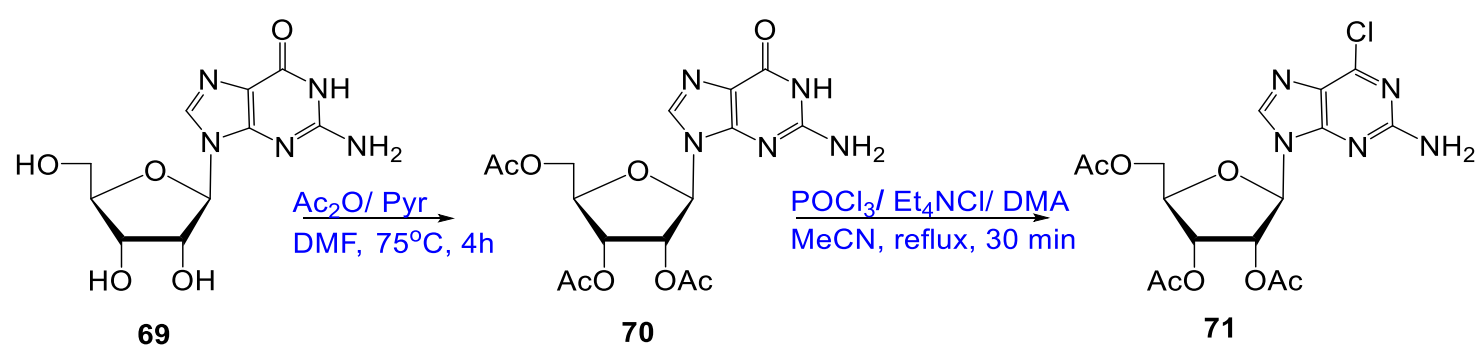

Scheme 25. Synthesis of protected 6-chloroadenosine from guanosine

Subsequently, treatment of $\mathbf{7 1}$ with benzyltriethylammonium nitrite $\left(\mathrm{BzEt}_{3} \mathrm{~N}-\mathrm{NO}_{2}\right)$ and trimethylsilyl chloride (TMS-Cl) gave a diazonium salt that was replaced with chlorine yielding 2,6-dichloro derivative 72 (58\%, Scheme 26). ${ }^{212}$ The 6-position chlorine was then replaced by amine using ammonia gas $\left(\mathrm{NH}_{3}\right)$ saturated in 1,2-dimethoxyethane to afford protected 2-chloroadenosine $\mathbf{7 3}(85 \%)$. The previously mentioned reaction afforded a small 
amount of partially deprotected $\mathbf{7 3}$, which could be used in the following reaction to yield fully deprotected 2-chloroadenosine $\mathbf{7 4}$. It is noteworthy that the reaction of $\mathbf{7 2}$ using $\mathrm{MeOH} / \mathrm{NH}_{3}$ gave some of amine substituted product 73 , along with a large amount of 6position methoxy substituted byproduct. Deprotection of $\mathbf{7 3}$, fully or partially protected, using $\mathrm{MeOH} / \mathrm{NH}_{3}$ gave 2-chloroadenosine $74(95 \%) .{ }^{211}$

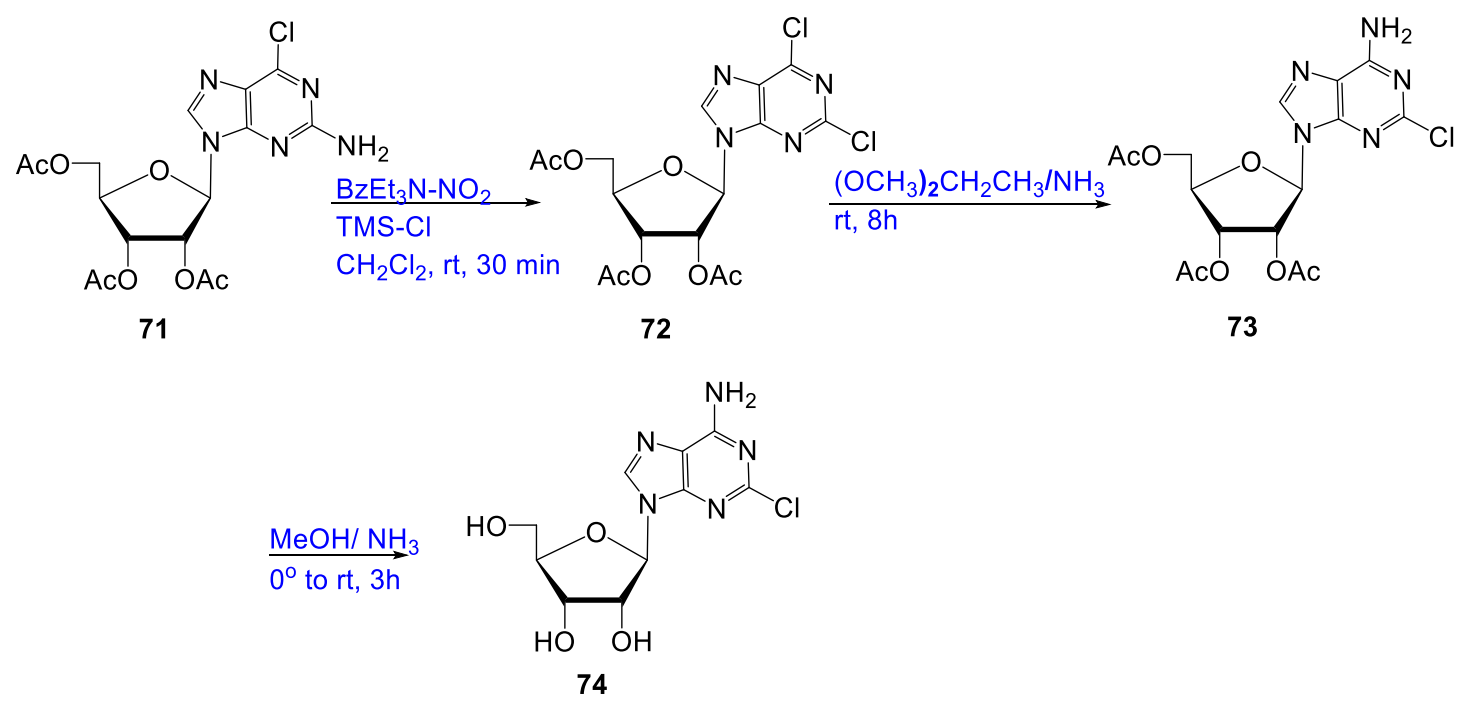

Scheme 26. Transformation of 6-chloro-2-aminopurine nucleoside to 2-chloroadenosine

Next, we attempted transform 2-chloroadenosine $\mathbf{7 4}$ to clofarabine $\mathbf{7 7}$ by regioselective protection of the sugar 3 ',5'-OH groups and 6- $\mathrm{NH}_{2}$ group using $\mathrm{TrCl} /$ pyridine/DMAP at $80{ }^{\circ} \mathrm{C}$ followed by stereoselective fluorination (Scheme 27). ${ }^{214}$ After 96 hours, the reaction mixture showed presence of $\mathbf{7 5 a}(\mathrm{R}=\mathrm{Tr})$ but in low yield $(<5 \%$ by TLC), along with several partially protected byproducts, which required extensive column chromatography for separation. Alternatively, protection of 3',5'-OH and 6- $\mathrm{NH}_{2}$ was attempted using benzoyl chloride $(\mathrm{BzCl})$ pyridine at $-5{ }^{\circ} \mathrm{C}$ for 30 mins to afford $\mathbf{7 5 b}(\mathrm{R}=\mathrm{Bz})$ in very low yields (5\% after column chromatography), with several byproducts. Due to the low efficiency of the reactions leading up to compound $\mathbf{7 5}$, we then explored methods for a more rapid and 
efficient synthesis of clofarabine 77 employing coupling of commercially available 1,3,5tri- $O$-benzoyl-2-deoxy-2-fluoro- $\alpha$-D-arabinofuranose with 2-chloroadenine base. ${ }^{216}$

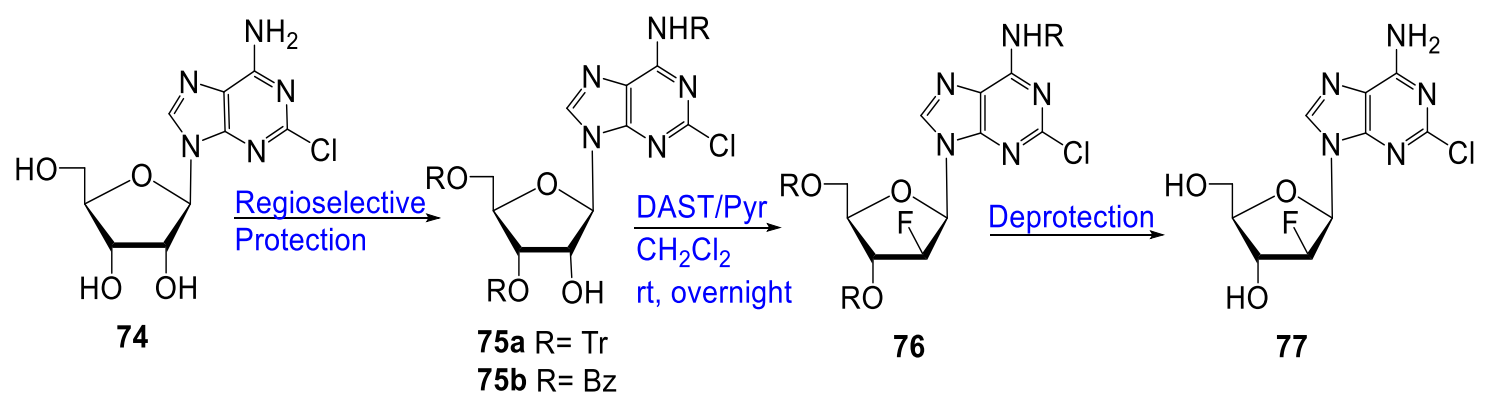

Scheme 27. Attempted synthesis of clofarabine from 2-chloroadenosine

\subsubsection{Synthesis of clofarabine by sugar/base coupling}

Bromination of the 1 position of commercially available 1,3,5-tri- $O$-benzoyl-2-deoxy2-fluoro- $\alpha$-D-arabinofuranose 78 using $\mathrm{HBr}$ gave pure 2-deoxy-1- $\alpha$-bromo-2- $\beta$-fluoro-3,5di-O-benzoyl-d-ribofuranose 79 (99\%, Scheme 28). Subsequent coupling of 79 with 2chloroadenine in the presence of $\mathrm{KO} t-\mathrm{Bu} / \mathrm{CaH}_{2}$ afforded a mixture of protected $\beta$ clofarabine $80(55 \%)$ and its $\alpha$-anomer 80a (40\%). Addition of a drying agent, such as $\mathrm{CaH}_{2}$, and use of a polar aprotic solvent like $\mathrm{MeCN}$ led to the higher yield of $\beta$-isomer. Finally, deprotection of $\mathbf{8 0}$ with $\mathrm{NaOMe} / \mathrm{MeOH}$ afforded clofarabine 77 (95\%, overall: $52 \%$ from 78$).{ }^{216}$ 


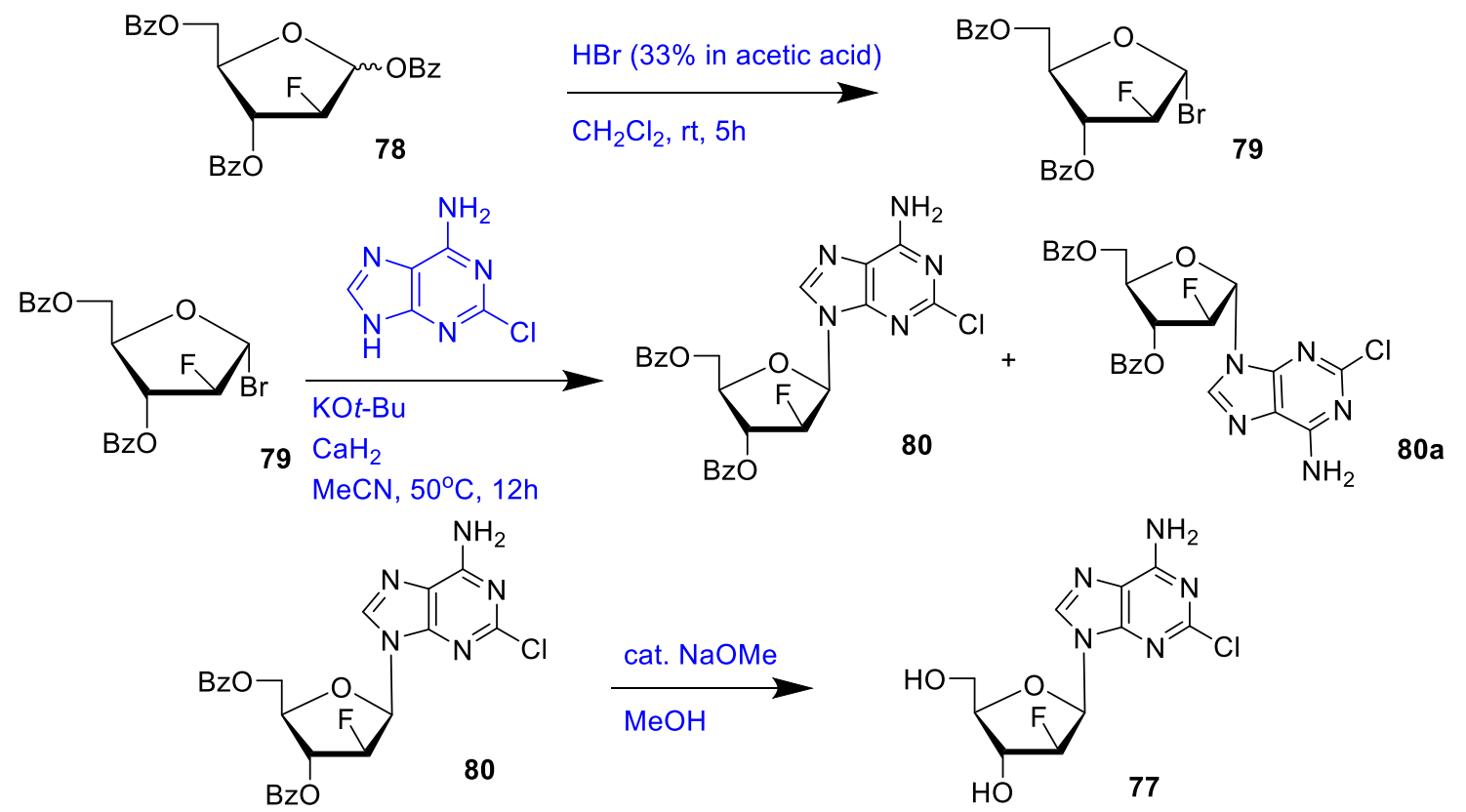

Scheme 28. Synthesis of clofarabine by coupling of 2-deoxy-1- $\alpha$-bromo-2- $\beta$-fluoro-3,5di- $O$-benzoyl- $\beta$-ribofuranose to 2 -chloroadenine

\subsubsection{Synthesis of clofarabine 5'-monophosphate}

Synthesis of clofarabine 5'-monophosphate triethylammonium acetate salt $\mathbf{8 1}$ was achieved using two methods: (i) Standard Yoshikawa method $\left(\mathrm{POCl}_{3} / \mathrm{PO}(\mathrm{OMe})_{3}\right)^{15}$ and (ii) Modified Yoshikawa method in the presence Proton Sponge. ${ }^{210,217}$ These reactions were attempted under ambient conditions, or anhydrous conditions with freshly distilled $\mathrm{POCl}_{3}$. Ultimately, it was found that use of distilled $\mathrm{POCl}_{3}$ in anhydrous conditions, using standard Yoshikawa method was the most successful producing $\mathbf{8 1}$ in high yield with less rigorous purification needed (Scheme 29). Thus, treatment of clofarabine 77 in $\mathrm{PO}(\mathrm{OMe})_{3}$ at $0{ }^{\circ} \mathrm{C}$ with $\mathrm{POCl}_{3}$ followed by Sephadex column with TEAB buffer, and HPLC purification yielded pure $\mathbf{8 1}$ in $35 \%$ yield (Figure 43 ). 

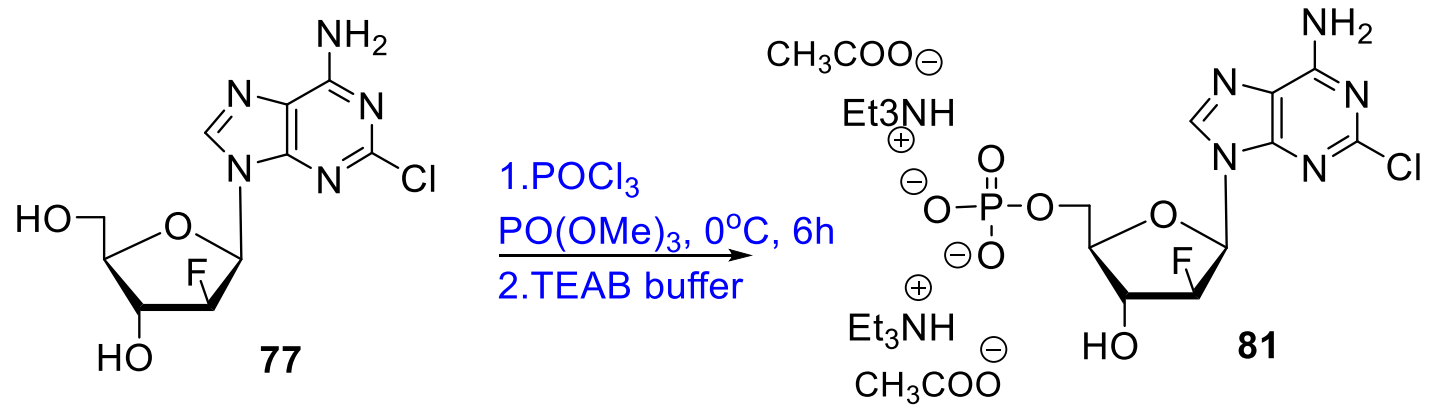

Scheme 29. Phosphorylation of clofarabine using Yoshikawa method

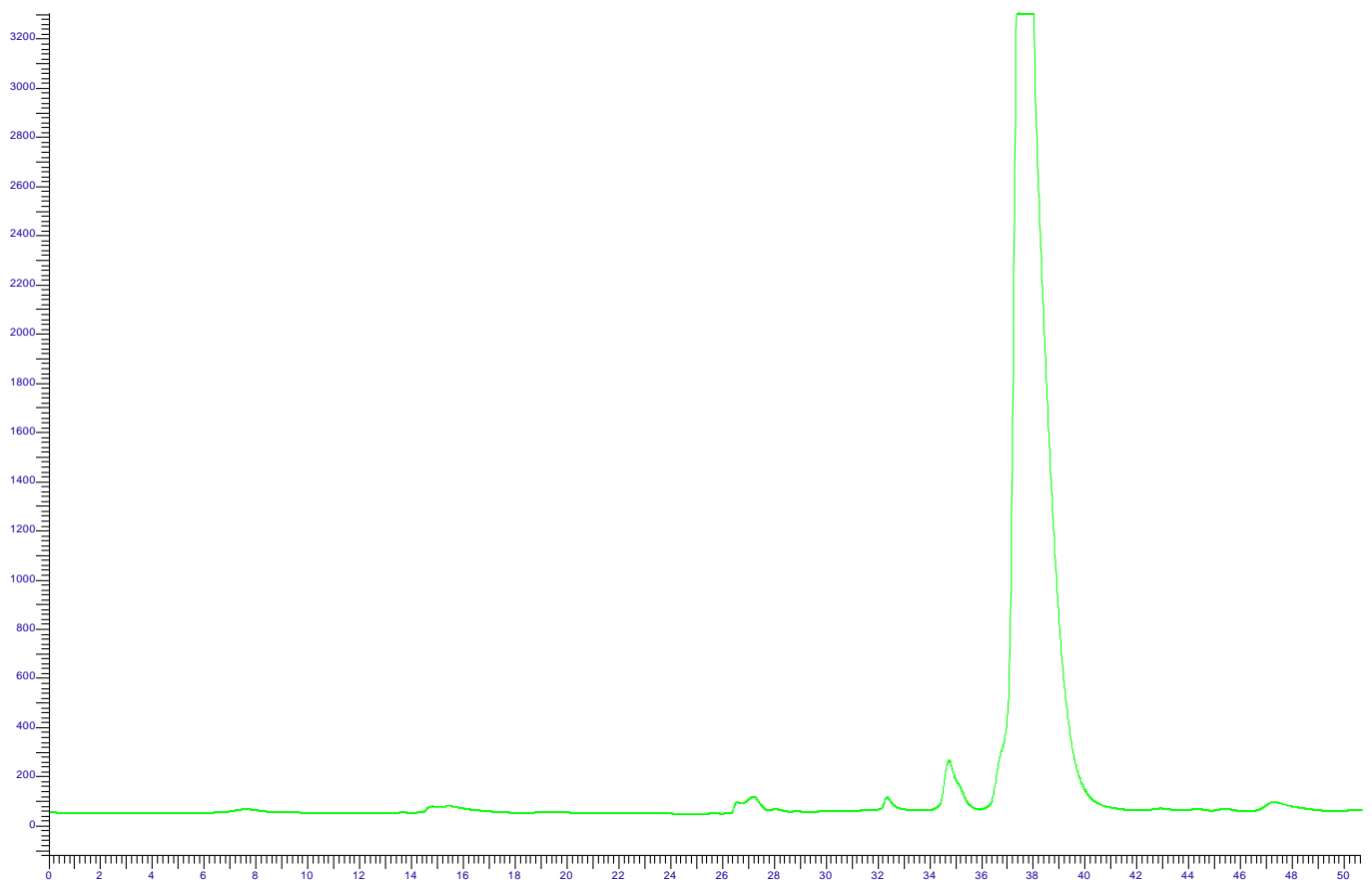

Figure 43. HPLC purification of clofarabine 5'-monophosphate

\subsubsection{Symmetric phosphoramidites}

Although asymmetric bis(acyloxybenzyl) phosphoramidites were found to be much more effective in releasing the unmasked diphosphate without cleavage to the monophosphate, ${ }^{51}$ we initially explored synthesis of symmetric phosphoramidites to become familiar with the protocol and chemistry. To acquire symmetric phosphoramidites to use in coupling to clofarabine 5'-monophosphate, benzoyloxy benzyl alcohol $\mathbf{8 3}$ was synthesized by treatment of 4-hydroxybenzyl alcohol $\mathbf{8 2}$ with benzoyl chloride $(\mathrm{BzCl})$ in 
the presence of $\mathrm{Et}_{3} \mathrm{~N}$ in THF at $-20{ }^{\circ} \mathrm{C}$ for 2 hours $(50 \%$, Scheme 30$)$. The moderate yield was attributed to the formation of dibenzoylated byproduct, and some unreacted starting material.

Then, dry solid 83 was reacted with anhydrous $\mathrm{Et}_{3} \mathrm{~N}$ and $\mathrm{PN}(i \mathrm{Pr})_{2} \mathrm{Cl}_{2}$, under nitrogen, in THF at $-20{ }^{\circ} \mathrm{C}$ for 30 mins to yield bis(benzoyloxybenzyl) phosphoramidite $84\left[{ }^{31} \mathrm{P}\right.$ NMR $\delta 147.9$ ppm; Figure 44$].{ }^{50}$ Purification of this compound using a $2 \% \mathrm{Et}_{3} \mathrm{~N}$ in hexane silica gel column was explored with varied success. Larger scale reactions (>200mg) proved much more successful in yield and purification. However, crude product $\mathbf{8 4}$ was used in coupling reactions for simplicity and reproducibility purposes. ${ }^{47,53}$

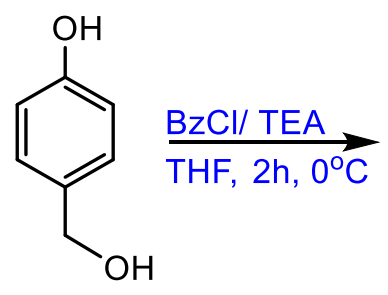

82<smiles>O=C(Oc1ccc(CO)cc1)c1ccccc1</smiles>

83
TEA
$\underset{\mathrm{PN}(i \mathrm{Pr})_{2} \mathrm{Cl}_{2}}{\mathrm{THF}, 30 \mathrm{~min},-20^{\circ} \mathrm{C}}$<smiles>CN([N+](=O)[O-])P(O)OCc1ccc(OC(=O)c2ccccc2)cc1</smiles><smiles>COCc1ccc(OC(=O)c2ccccc2)cc1</smiles>

Scheme 30. Synthesis of bis(benzoyloxybenzyl) phosphoramidite 


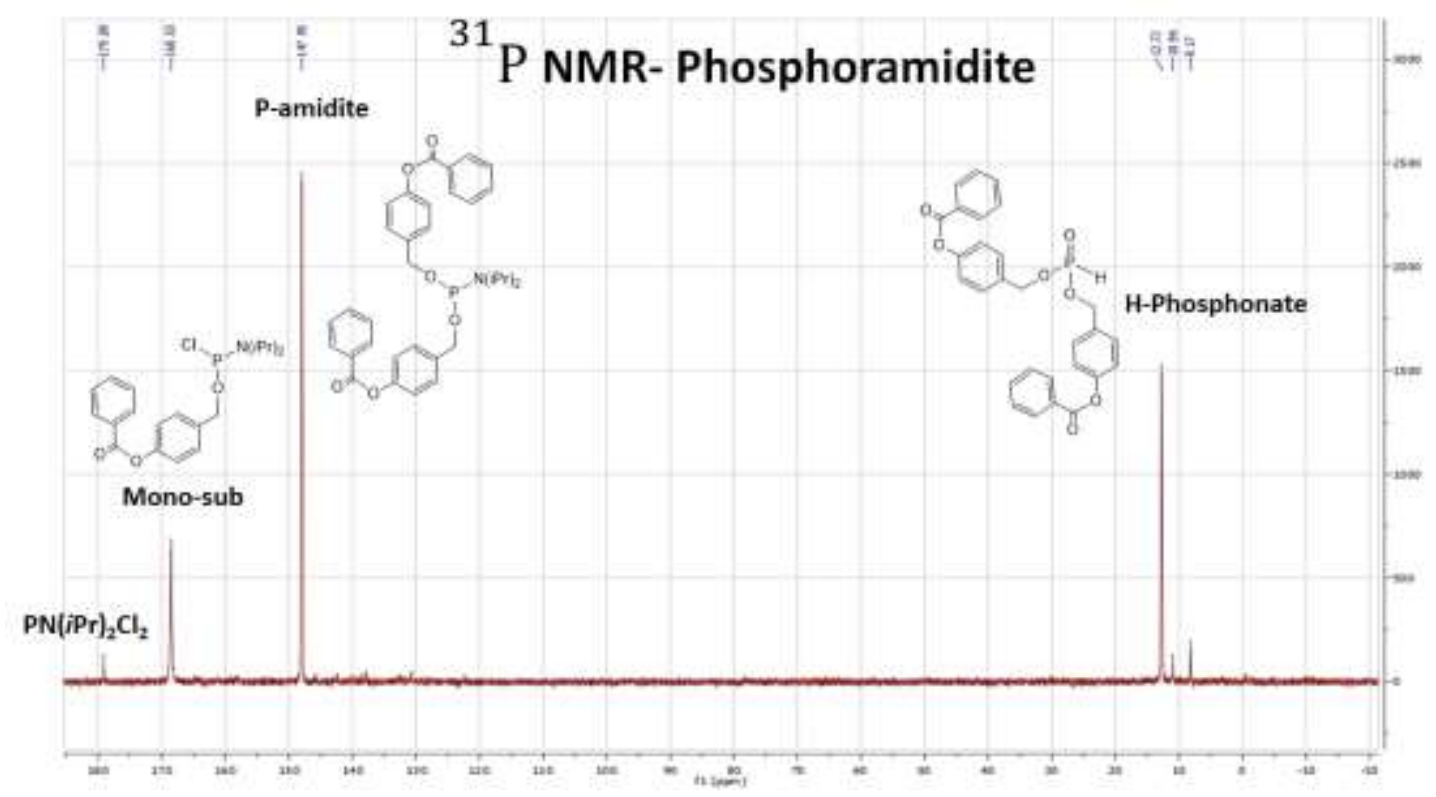

Figure 44. ${ }^{31} \mathrm{P}$ NMR of crude bis(benzoyloxybenzyl)phosphoramidite

\subsubsection{Model studies: adenosine 5'-diphosphate prodrug}

To determine a proper method for synthesis of clofarabine 5'-diphosphate prodrugs (DiPPro), model studies were conducted using adenosine $\mathbf{8 5}$ and simple symmetric phosphoramidite unit $\mathbf{8 4}$. Synthesis of prodrug $\mathbf{8 7}$ began by monophosphorylation of adenosine 85 using Yoshikawa method $\left[\mathrm{POCl}_{3} / \mathrm{PO}(\mathrm{OMe})_{3}\right]^{15}$ at $0^{\circ} \mathrm{C}$ for 6 hours to yield adenosine 5'-monophosphate triethyl ammonium salt $\mathbf{8 6}$ after Sephadex purification (Scheme 31). Alternatively, commercially available adenosine 5'-monophosphate sodium salt 86a was converted to 86, using Dowex $(\mathrm{H}+) / \mathrm{TEAB}$ buffer, to increase solubility in organic solvent like DMF. 

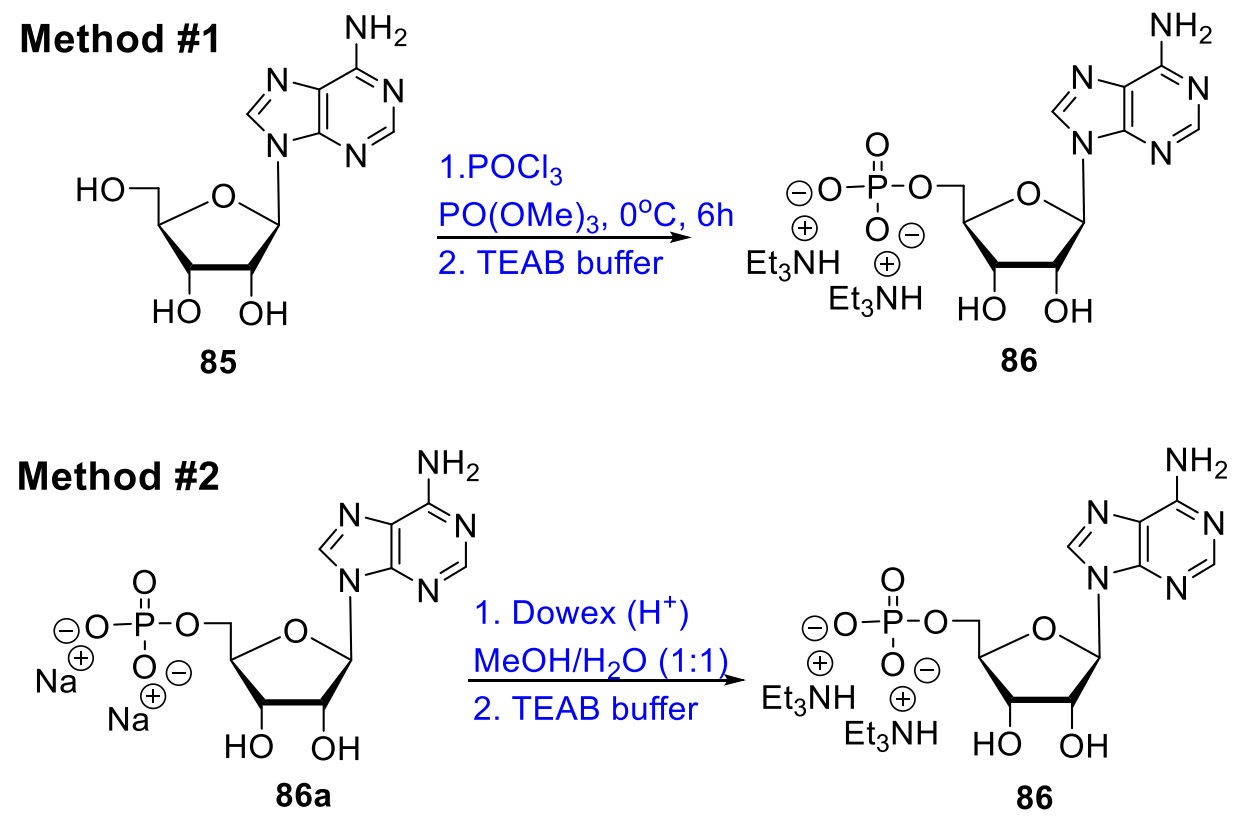

Scheme 31. Synthesis of adenosine 5'-monophosphate triethylammonium salt

Next, phosphoramidite 84 was coupled with adenosine 5'-monophosphate salt 86 (Scheme 32) using 5-phenyl-1- $H$-tetrazole activator ${ }^{218}\left[{ }^{31} \mathrm{P}\right.$ NMR for $\mathrm{P}(\mathrm{III}) \delta 126.2 \mathrm{ppm}$, $\mathrm{P}(\mathrm{V}) \delta$-9.7 ppm; Figure 46 Panel A], followed by oxidation of the resulting 87a with $t$ - $\mathrm{BuOOH}^{53}$ to form adenosine $5^{\prime}$-diphosphate prodrug $87\left[{ }^{31} \mathrm{P}\right.$ NMR $\delta-12.07 \mathrm{ppm}(\mathrm{d}, J=$ 19.8 Hz, $\mathrm{P}_{\alpha}$ ), $-12.71\left(\mathrm{~d}, J=19.8 \mathrm{~Hz}, \mathrm{P}_{\beta}\right)$; Figure 46 Panel B]. Coupling of 84 with 86 was attempted using 4,5-dicyanoimidazole (DCI) as previously reported for other diphosphate prodrugs $;{ }^{50-53}$ however, this method yielded a complex reaction mixture along with some starting material. Purification of $\mathbf{8 7}$ proved to be quite difficult, with decomposition back to the adenosine 5'-monophosphate observed during HPLC purification using both $\mathrm{MeCN} / \mathrm{H}_{2} \mathrm{O}$ systems and 20mM TEAA buffer systems (Figure 45). Crystallization using ethyl acetate proved the most effective method for purification providing $\mathbf{8 7}$ with $95 \%$ purity as analyzed by NMR. 


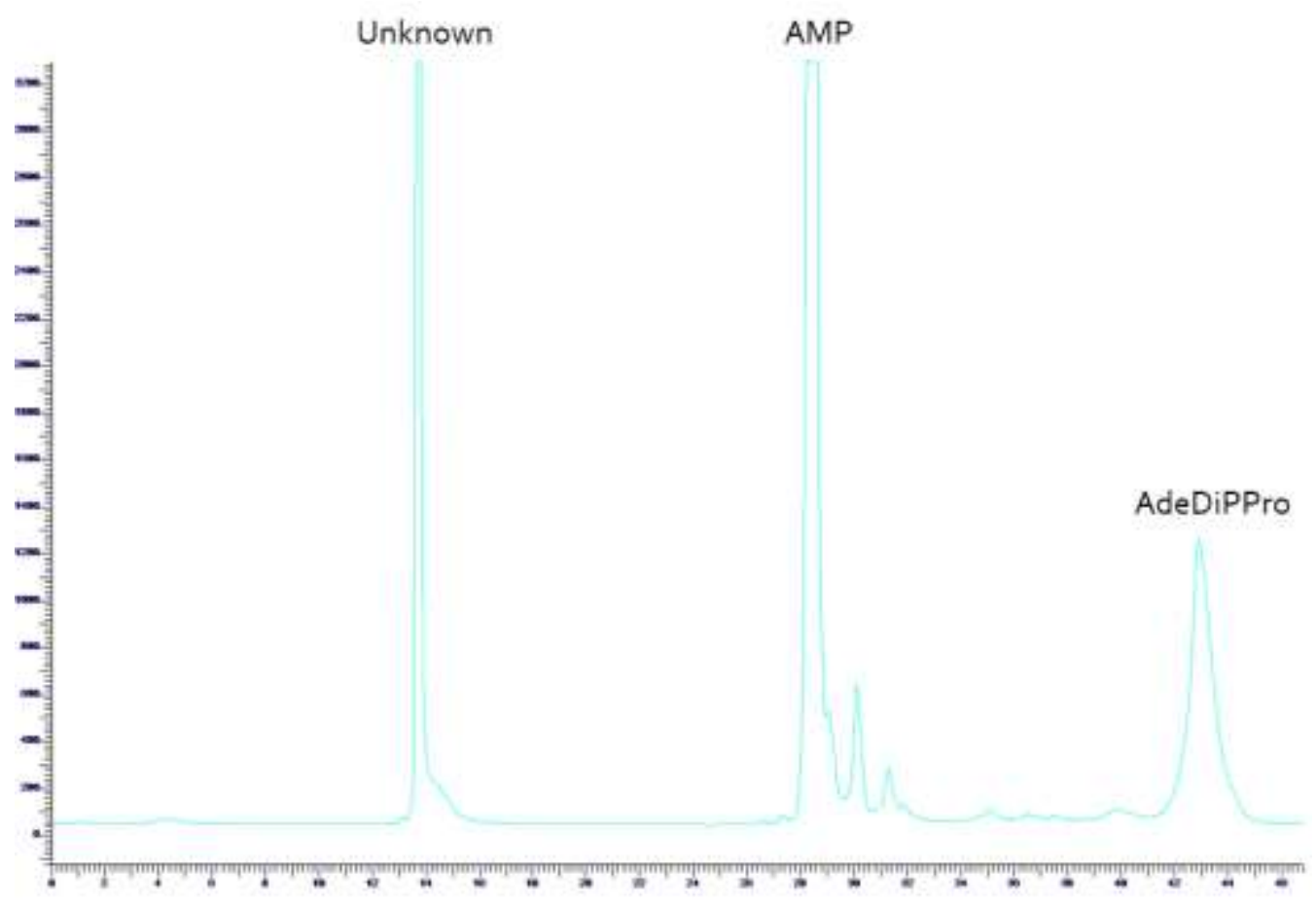

Figure 45. HPLC purification of DiPPro 87 using $\mathrm{CH}_{3} \mathrm{CN} / \mathrm{H}_{2} \mathrm{O}$ system 

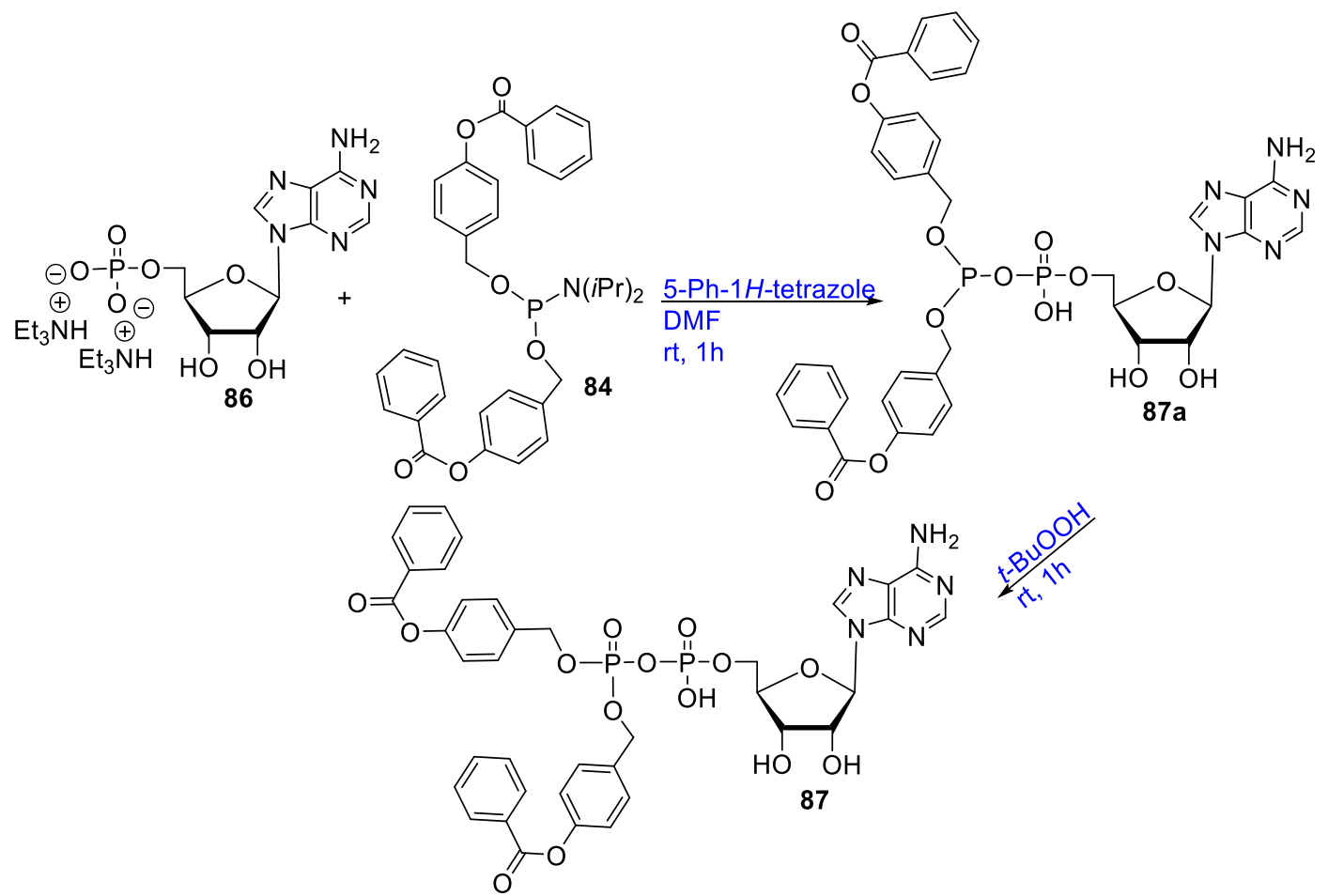

Scheme 32. Coupling of bis(benzoyloxybenzyl) phosphoramidite to adenosine 5'monophosphate 

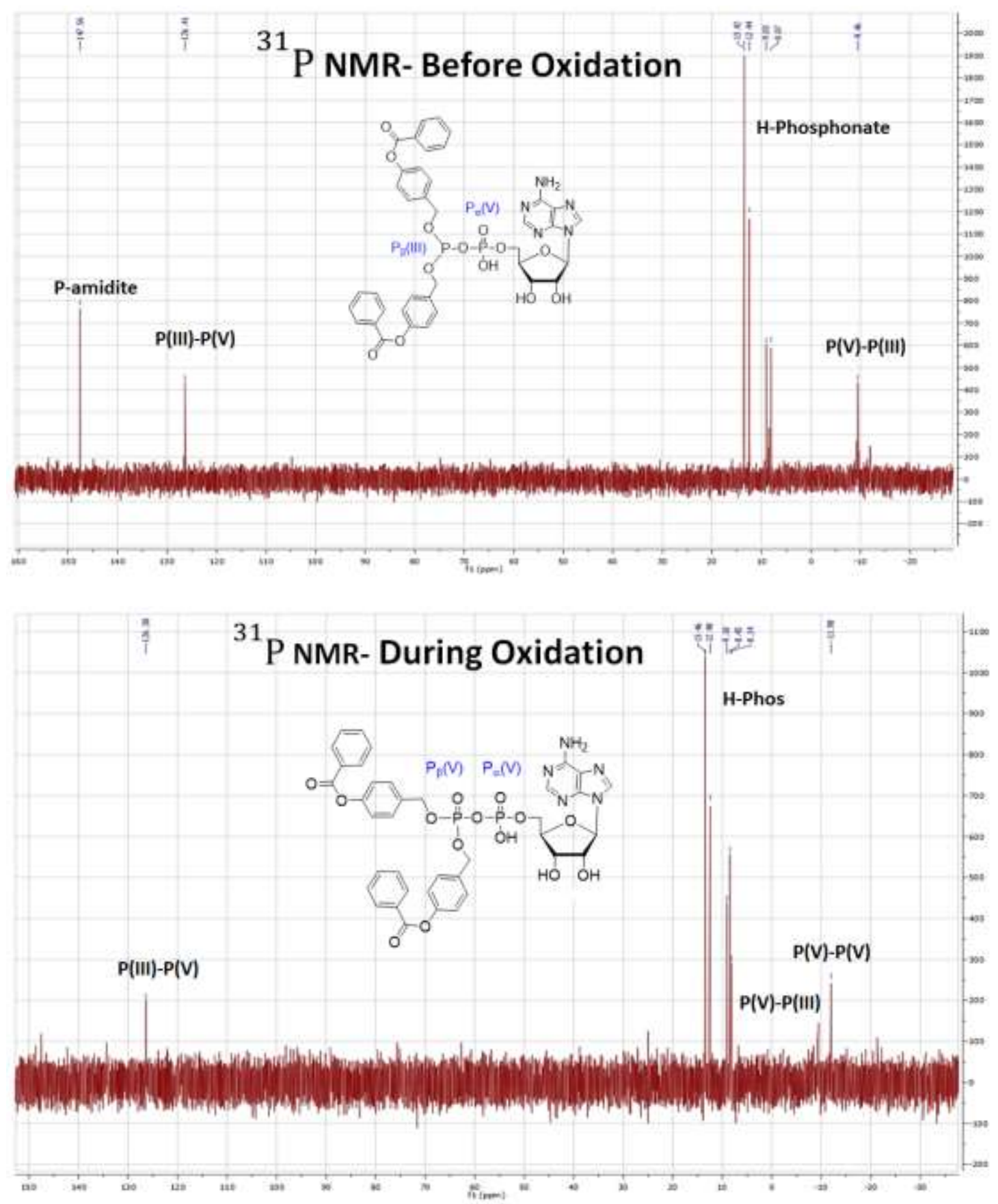

Panel A: formation of new $\mathrm{P}(\mathrm{III})-\mathrm{P}(\mathrm{V})$ bond in 87a with peaks at $126 \mathrm{ppm}$ and -9 ppm; Panel B: displays formation of new $\mathrm{P}(\mathrm{V})-\mathrm{P}(\mathrm{V})$ bond in $\mathbf{8 7}$ showing two doublets in the -12 ppm region

Figure 46. ${ }^{31} \mathrm{P}$ NMR analysis of the formation of adenosine DiPPro before and after oxidation. 


\subsubsection{Attempted synthesis of clofarabine 5'-diphosphate ProTides}

Given our success with the adenosine DiPPro model 87, coupling of phosphoramidite 84 to clofarabine 5'-monophosphate triethylammonium acetate salt $\mathbf{8 1}$ was attempted using 5-phenyl-1- $H$-tetrazole ${ }^{218}$ as an activator in DMF, followed by oxidation using $t$-BuOOH. ${ }^{53}$ This was expected to yield clofarabine 5'-diphosphate prodrug $\mathbf{8 8}$ (Scheme 33) in similar yield to adenosine prodrug $\mathbf{8 7}$. However, the results for this reaction showed low yields of $\mathbf{8 8}$ as evidenced by lack of formation of two doublets in the $-12 \mathrm{ppm}$ region in ${ }^{31} \mathrm{P}$ NMR spectra in higher than 3-5\%. A large amount of unreacted starting material 81 was seen with ${ }^{31} \mathrm{P}$ NMR peaks in the $2 \mathrm{ppm}$ region, along with byproducts in the 8-10 ppm region which may be identified as H-phosphonate byproducts of $\mathbf{8 4}$.

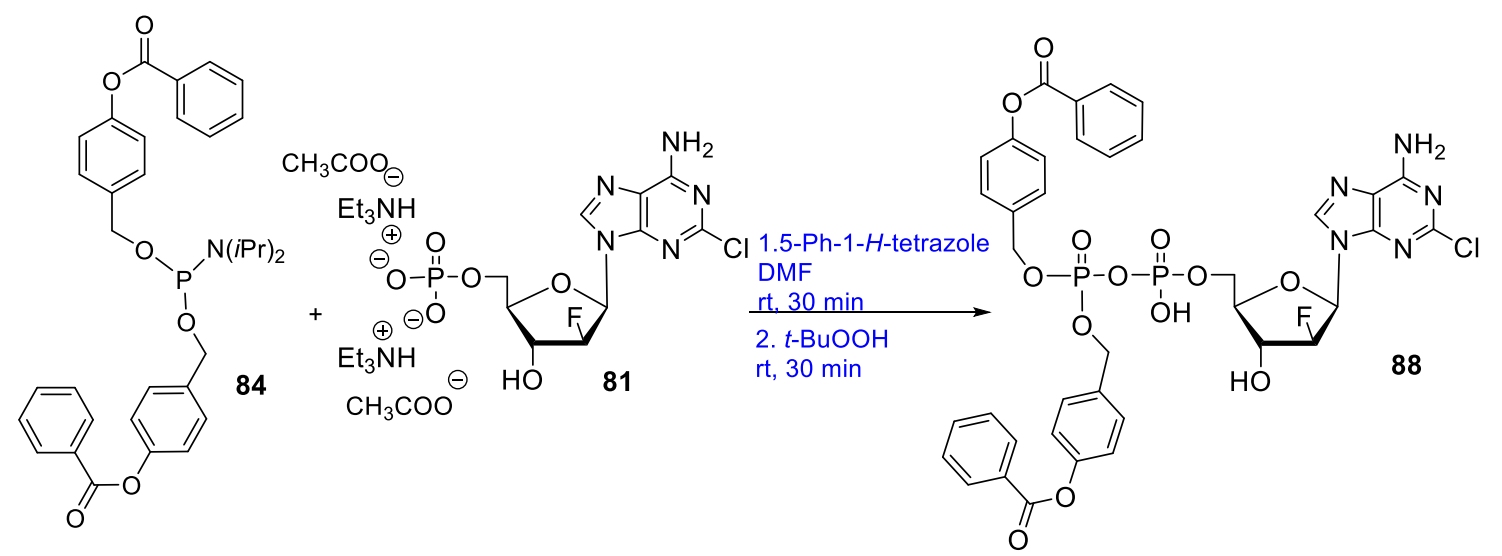

Scheme 33. Attempted coupling of symmetric phosphoramidite to clofarabine 5'monophosphate

Changes in synthesis/purification of clofarabine 5'-monophosphate $\mathbf{8 1}$ and phosphoramidite $\mathbf{3 4}$ may be needed to yield desired $\mathbf{8 8}$. The presence of the acetate anion in 81, which is formed by HPLC purification with tetraethylammonium acetate (TEAA) buffer, may discourage nucleophilic attack of phosphate anion towards phosphoramidite 84. This can explain the presence of starting material and byproducts after coupling 
reactions. It may be beneficial to explore conversion of $\mathbf{8 1}$ to triethylammonium salt $\mathbf{8 1 a}$ using Dowex H+/TEAB buffer (Scheme 34), and re-attempted coupling (Scheme 35).

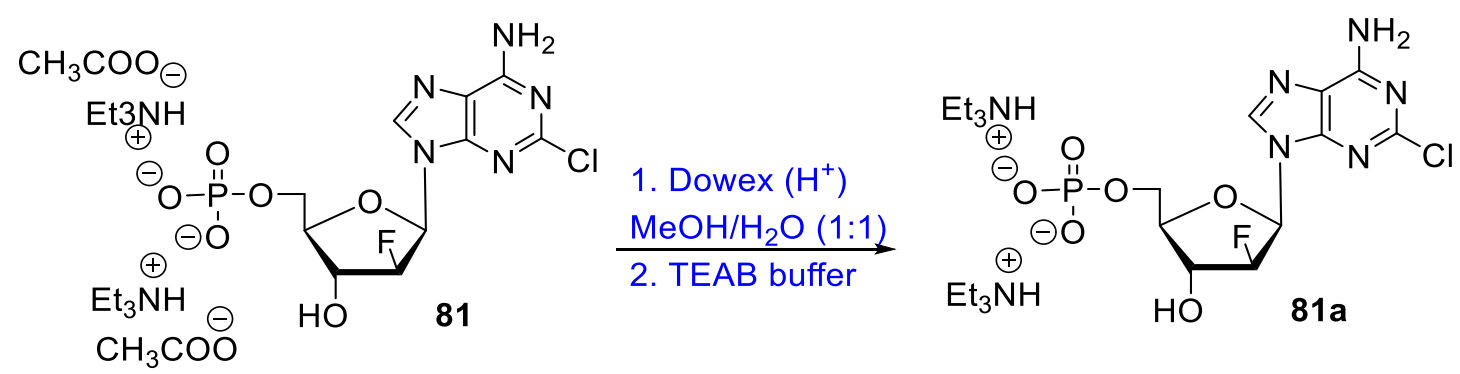

Scheme 34. Conversion of monophosphate counterions in clofarabine 5'-monophosphate

Although using crude phosphoramidite $\mathbf{8 4}$ in coupling reactions with adenosine 5'monophosphate 86 was successful, to prevent formation of byproducts, purification of $\mathbf{8 4}$ may be advantageous. The phosphoramidite $\mathbf{8 4}$ is not stable for column chromatography, but $\mathrm{Et}_{3} \mathrm{~N}$ can be used to avoid decomposition to $\mathrm{H}$-phosphonate. We have obtained varied results using $2 \% \mathrm{Et}_{3} \mathrm{~N}$ in hexane columns; with larger scale reactions showing less decomposition. It might be beneficial to use a higher amount of $\mathrm{Et}_{3} \mathrm{~N}$ for purification (i.e. 5\%) and prevent decomposition and achieve better reproducibility.

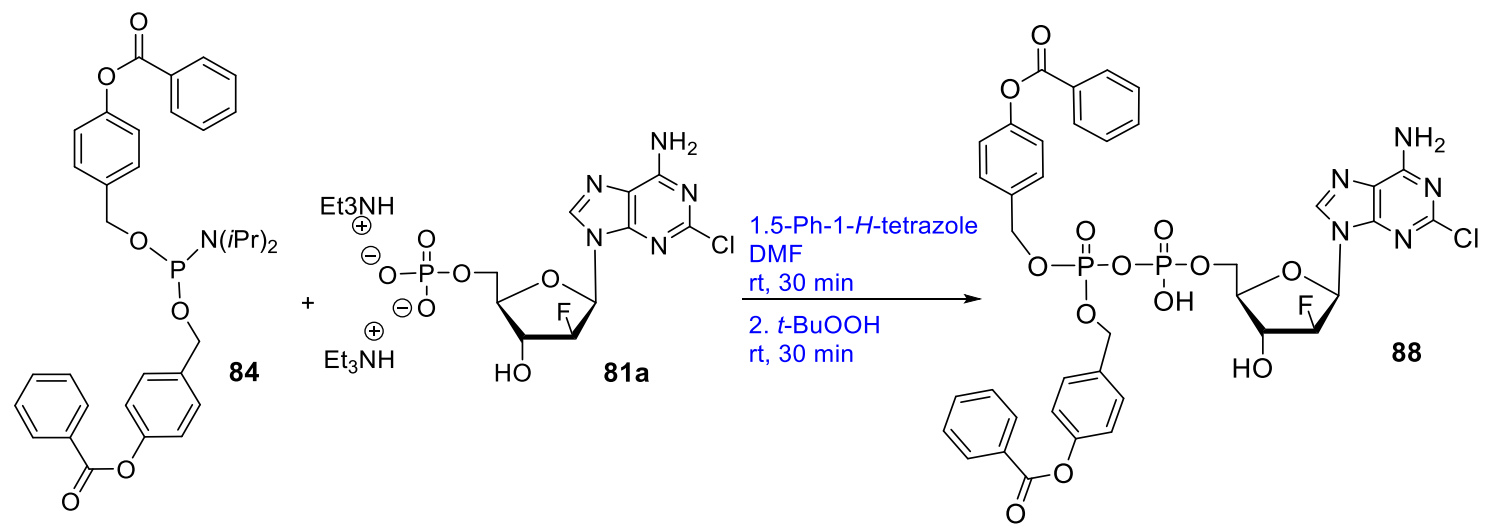

Scheme 35. Improved synthesis of clofarabine 5'-diphosphate prodrugs 


\subsection{Synthesis of azido-modified nucleosides for elucidation of structure of aminyl radicals}

\subsubsection{Synthesis of 2-azidopurine nucleosides for elucidation of the guanyl aminyl radical}

The guanyl aminyl radical $(\mathrm{G}(\mathrm{N} 2-\mathrm{H}) \cdot)$, as explained in Section 1.4 .1 of this dissertation, is of extreme importance in the study of DNA damage and repair pathways. This radical has been characterized by EPR spectroscopy in dsDNA-oligomers but not unequivocally in 2'-deoxyguanosine (dGuo) monomers. ${ }^{90-93,95,219}$ Characterization of the $(\mathrm{G}(\mathrm{N} 2-\mathrm{H}) \bullet)$ at all level DNA-models (i.e. monomers, oligomers, quadruplexes, etc.) is of high importance in understanding the chemistry of this radical in DNA. The EPR spectrum of an aminyl radical via radiation-produced electron-addition to a nucleoside was first discovered from 3'-AZT (3'-azido-3'-deoxythymidine), whose azido group present at the 3' position of the sugar can form a reactive aminyl radical (for mechanism see Section 1.4). ${ }^{104}$

The high-electron affinity of the azide moiety makes it the most likely to capture an electron during irradiation. Given this reactivity, and our lab's previous experience in the synthesis azido-modified carbohydrate and nucleoside analogues, ${ }^{105,106}$ we proposed the synthesis of 2-azido-2'-deoxyinosine, and other 2-azido substituted purines, in order to elucidate the structure of the elusive aminyl radical on the guanine base by EPR (Figure 47). 


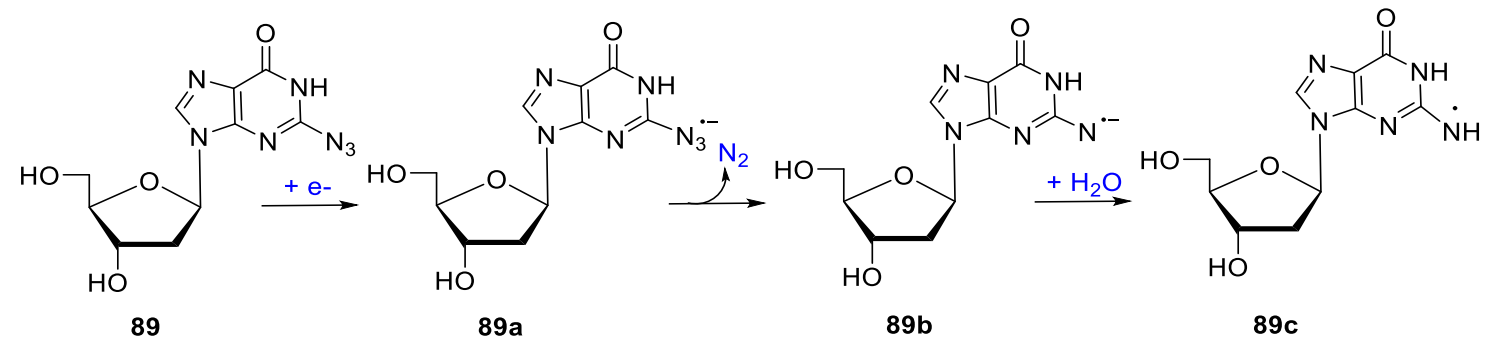

Figure 47. Proposed mechanism for the formation of aminyl radical in 2-azido-2'deoxyinosine upon irradiation with solvated electrons

We hypothesize that the azido group present at the C2-position of inosine will undergo a similar mechanism to that of the azide in $3^{\prime}-\mathrm{AZT}^{104}$ and AmdU. ${ }^{106}$ The azide will capture an electron forming an unstable azide anion radical $\left(\mathrm{RN}_{3} \bullet^{-}, \mathbf{8 9} \mathbf{a}\right)$, which will then lose $\mathrm{N}_{2}$, leaving a nitrene anion radical $\left(\mathrm{RN}^{\bullet^{-}}, \mathbf{8 9 b}\right)$. This nitrene anion radical will then abstract a hydrogen to become an aminyl radical $(\mathrm{RNH} \bullet, 89 \mathrm{c})$. Reactive aminyl radicals have been shown to cause DNA strand breakage via free radical reactions. ${ }^{85,220}$ Thus, if the structure of the reactive aminyl radical is determined by EPR, we would like to explore $\mathbf{8 9}$ as anticancer radiotherapy agent.

\subsubsection{Synthesis of 2-azidoinosine analogues from 2 -azidoadenosine analogues}

We explored synthesis of 2-azido-2'-deoxyinosine 89, also named in this dissertation as 2-azido-2'-deoxyguanosine because azido group in $\mathbf{8 9}$ can directly generate aminyl radical postulated to be formed from 2'-deoxyguanosine upon electron addition, ${ }^{120}$ in order to study closely the aminyl radical formed from 2'-deoxyguanosine in DNA. Retrosynthetic analysis of compound $\mathbf{8 9}$ (Figure 48), shows that it can be conveniently synthesized from 2-azido-2'-deoxyadenosine $\mathbf{9 0}$, which we can also investigate for the formation of aminyl radical for comparison purposes. An alternative synthesis of compound $\mathbf{8 9}$ was explored from $O^{6}$-NPE protected 2'-deoxyguanosine. The 2-azidoinosine derivative 91, deemed 2- 
azidoguanosine for our purposes, was synthesized from its respective 2-azidoadenosine precursor 92.

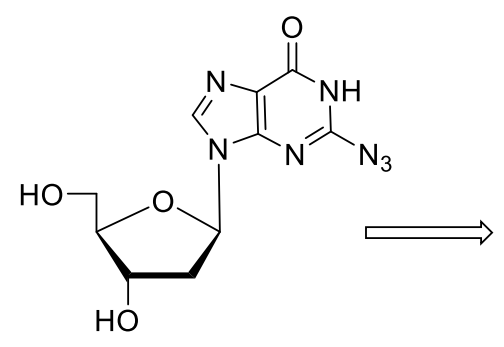

89

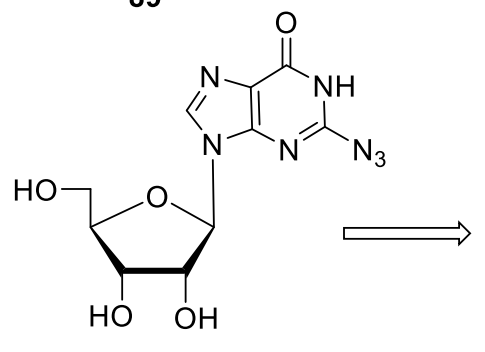

91

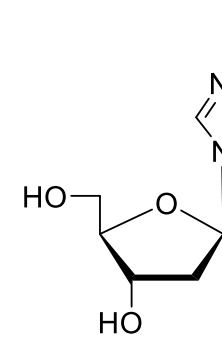

90

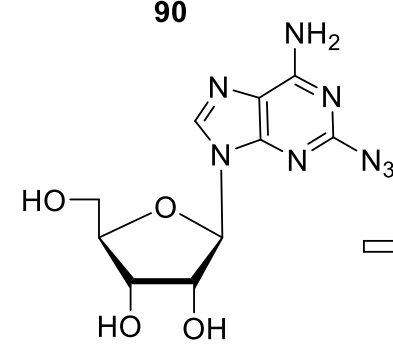

92

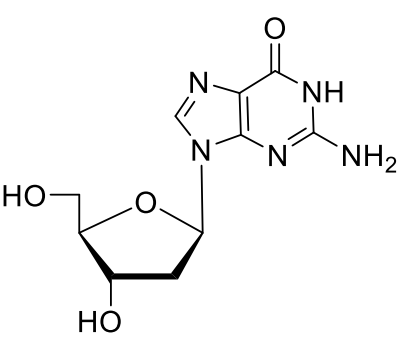

93

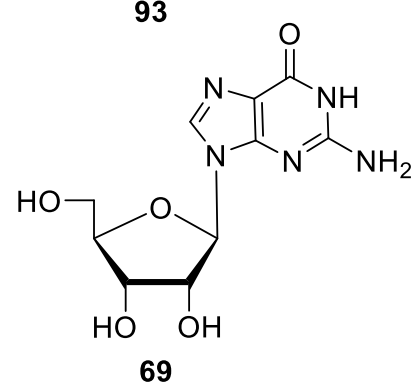

Figure 48. Retrosynthetic analysis of 2-azido-2'-deoxyinosine and 2-azidoinosine for the study of the elusive guanylyl aminyl radical

Analogue 89 was synthesized using literature protocols previously explored for the synthesis of clofarabine (e.g., Scheme 9 in Section 3.2.2.1). To begin with, 2'deoxyguanosine 93 was protected at the 3' and 5' sugar hydroxyls using $\mathrm{Ac}_{2} \mathrm{O} /$ pyridine to yield diacetylated 2'-deoxyguanosine 94 (70\%; Scheme 36). ${ }^{211}$ Lower yield was attained during acetylation mainly due to $\mathrm{N}$-acetylated byproduct as analyzed by TLC. Subsequent treatment of 94 with $\mathrm{POCl}_{3} / \mathrm{Et}_{4} \mathrm{NCl} / \mathrm{PhNMe}_{3}$ effected phosphorylation of the hydroxyl group at C6 (from tautomeric enol form), which releases a chloride anion that affects replacement at C6 giving 2-amino-6-chloropurine 95 (44\%). ${ }^{211,213}$ Conversion of guanylyl ring into a standard purine ring can greatly improve solubility in organic solvent, and thus reactivity. 

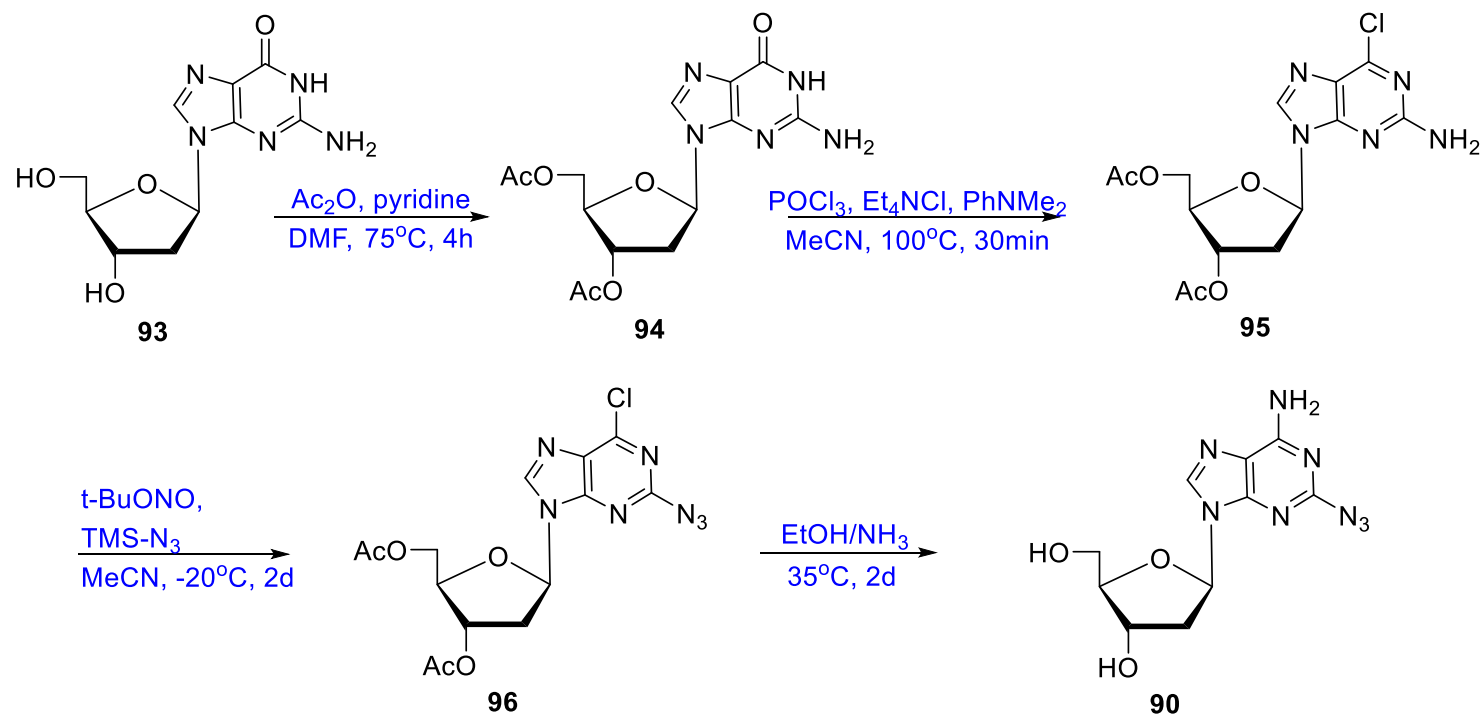

Scheme 36. Synthesis of 2-azido-2'-deoxyadenosine from 2'-deoxyguanosine

Diazotization of the 2-amino group in 95 with $t$-BuONO gave a diazonium salt, and subsequent replacement with azido group generated from TMS-N 3 reagent, afforded 2azido-6-chloropurine nucleoside 96 in $63 \%$ yield. Treatment of 96 with EtOH/NH afforded replacement of the 6-chloro with amine, and deprotection of the sugar hydroxyls to give 2-azido-2'-deoxyadenosine 90 (84\% yield). ${ }^{146}$ Finally, deamination of 90 with adenosine deaminase afforded 2-azido-2'-deoxyinosine $\mathbf{8 9}$ (85\%; Scheme 37). The reaction of 90 with adenosine deaminase was quite slow ( 7 days for $85 \%$ conversion), which is in agreement with slow deamination previously seen in 2-substituted adenosine analogues. ${ }^{221}$<smiles>Nc1nc(N)c2ncn(C3CC(O)C(CO)O3)c2n1</smiles>

\section{$\underset{\text { Adenosine Deaminase }}{\stackrel{\text { Phosphate Buff. } \mathrm{pH}=7.4}{\longrightarrow}}$} $28^{\circ} \mathrm{C}, 7 \mathrm{~d}$

Scheme 37. Synthesis of 2-azido-2'-deoxyinosine from 2-azido-2'-deoxyadenosine 
Alternatively, synthesis of 2-azido-2'-deoxyinosine $\mathbf{8 9}$ was attempted starting with diacetylated 2'-deoxyguanosine 94. This method involved the protection/activation of 6hydroxyl group in 94 with NPE. ${ }^{222,223}$ Thus, analogue 94 was treated with NPE-OH, triphenyl phosphine, and diisopropyl azodicarboxylate (DIAD) in dioxane at room temperature overnight to give 72\% yield of $O^{6}$-NPE-protected 2'-deoxyinosine 97 (Scheme 38). The use of molecular sieves powder in this reaction was imperative to the formation of product. Analogues $\mathbf{9 7}$ was then subjected to diazotization with $t$-BuONO reagent followed by displacement with azide generated from TMS- $\mathrm{N}_{3}$ reagent to afford $45 \%$ of 2azido compound 98. Finally, removal of $O^{6}$-NPE protection using 1,8diazabicyclo[5.4.0]undec-7-ene (DBU) followed by deprotection of sugar hydroxyls using $\mathrm{MeOH} / \mathrm{NH}_{3}$ afforded quantitative yield of 2-azido-2'-deoxyguanosine 89. It is noteworthy that performing deprotection steps subsequently without isolation of $\mathbf{9 9}$ yielded product $\mathbf{8 9}$ with some impurities. To ensure purity, $\mathbf{9 9}$ was column chromatographed after DBU deprotection $\left(10 \% \mathrm{MeOH} / \mathrm{CH}_{2} \mathrm{Cl}_{2}\right)$. After deprotection of sugar hydroxyls, HPLC purification (10\% $\mathrm{MeCN}$ in $\mathrm{H}_{2} \mathrm{O}$ system) of $\mathbf{8 9}$ provided a high purity sample for EPR studies. 


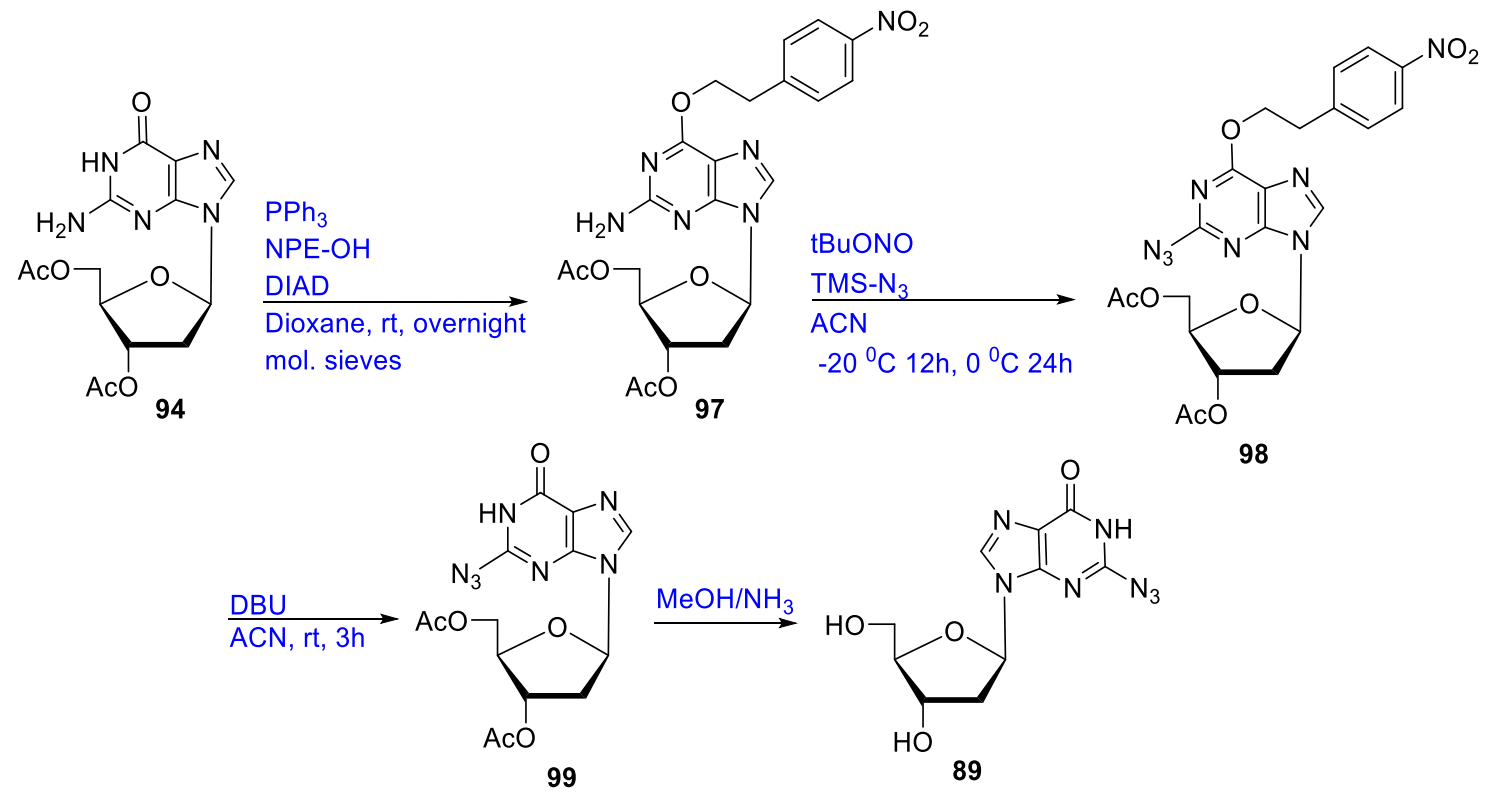

Scheme 38. Alternative synthesis of 2-azido-2'-deoxyinosine from 2'-deoxyguanosine

Analogous to the synthesis of $\mathbf{8 9}$, the 2-azidoguanosine derivative 91 was synthesized from 2-azidoadenosine $\mathbf{9 2}$. Synthesis of adenosine analogue 92 was achieved by formation of 6-chloro-2-amino protected purine 71, previously synthesized as a precursor to 2chloroadenosine in the route to clofarabine (e.g., Scheme 25 in Section 3.2.2.1). Thus, treatment of $\mathbf{7 1}$ with $t$-BuONO afforded diazonium salt which was replaced with azido group generated from TMS-N 3 to give 100 (78\%; Scheme 39). Deprotection and exchange of 6-chloro for amino group with $\mathrm{EtOH} / \mathrm{NH}_{3}$ gave 2-azidoadenosine 92 in $52 \%$ yield. ${ }^{146}$ This reaction gives a practical yield of $86 \%$ given the presence of partially deprotected byproducts, which upon reaction with fresh $\mathrm{EtOH} / \mathrm{NH}_{3}$ yielded additional amounts of product 92. Finally, adenosine deaminase was also used to convert 92 to 2-azidoguanosine $91(85 \%$, Scheme 40). Reaction of 2-azidoadenosine with adenosine deaminase was significantly faster than that of 2-azido-2'-deoxyadenosine (three days rather than seven days) as expected given the affinity of the enzyme. ${ }^{221}$ 


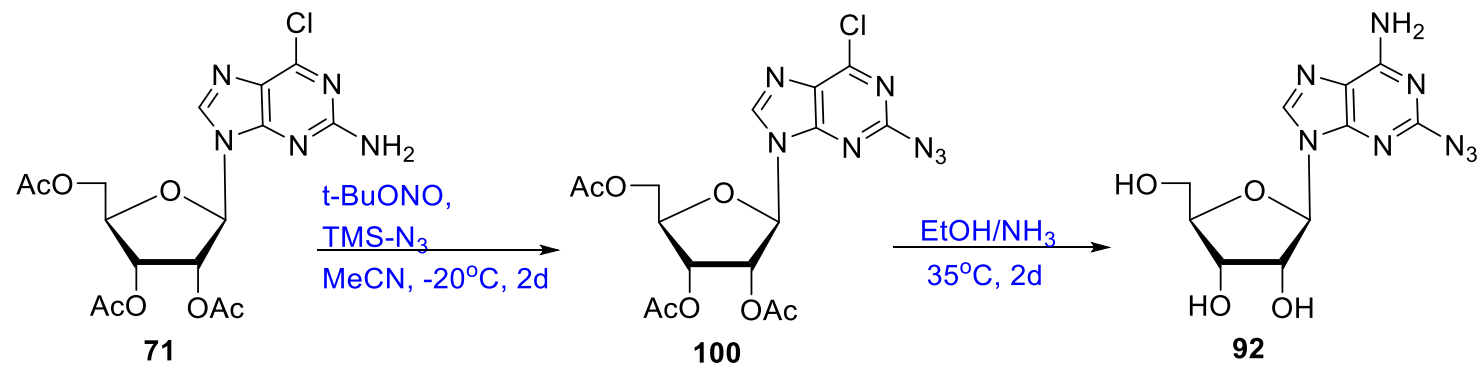

Scheme 39. Synthesis of 2-azidoadenosine from 6-chloro-2-amino protected purine

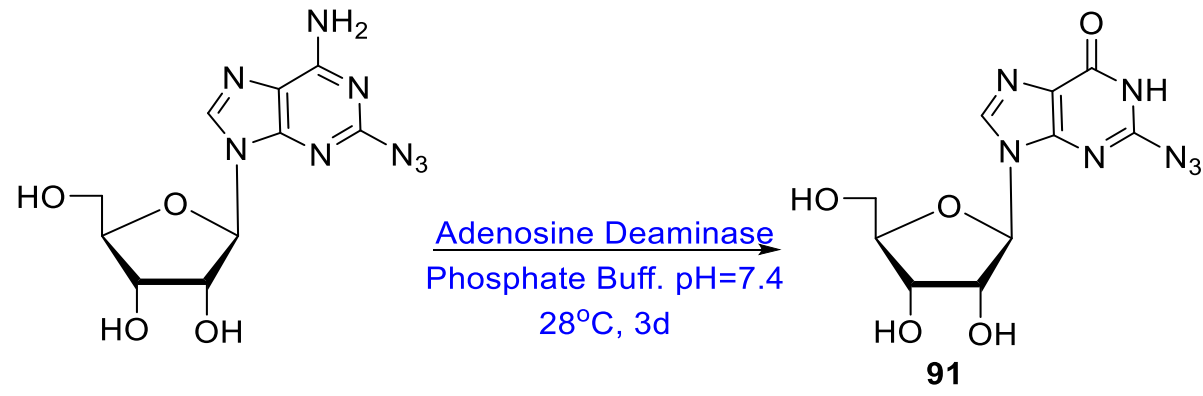

Scheme 40. Synthesis of 2-azidoguanosine from 2-azidoadenosine

Interestingly, 2-azidoadenosine analogues (e.g., 90 or 92) showed two sets of peaks in ${ }^{1} \mathrm{H}$ NMR, unlike 2-azidoinosine analogues which only displayed one set of peaks. The two sets of signals in 2-azidoadenosine analogues has been previously reported and highlight the tautomerization into their respective tetrazolo isomer (e.g., 90a and 92a; Scheme 41). The more deshielded peaks in NMR spectra were assigned to the tetrazole isomer. ${ }^{141,142}$ We were then interested in the effect that the stable tetrazole might have on formation of the aminyl radical upon electron addition. Azido analogues were sent to our collaborators in Oakland University to study formation of radicals upon irradiation with solvated electrons and to conduct EPR studies. 


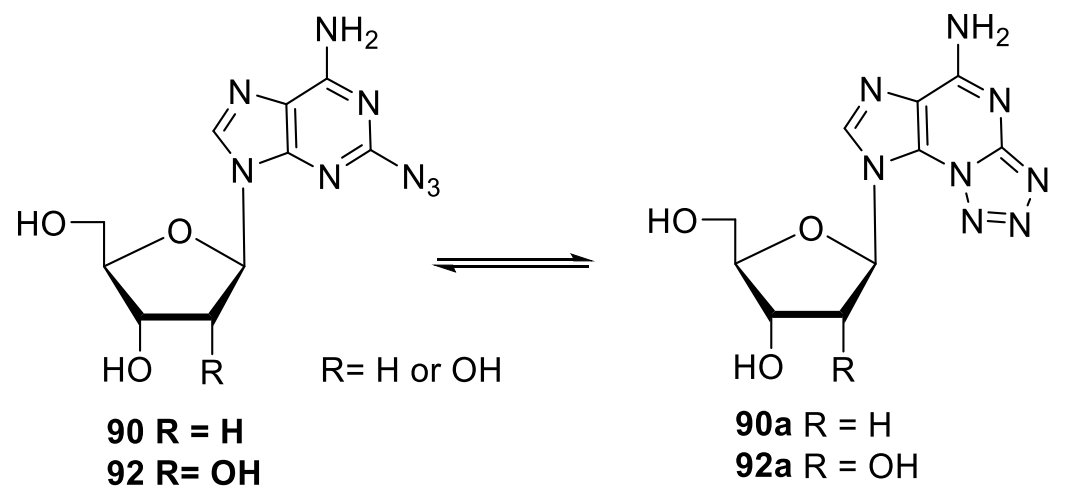

Scheme 41. Tautomerization of 2-azidoadenosine analogues

\subsubsection{Synthesis of 6-azidopurine nucleoside to study adenylyl aminyl radical}

We then set our sights on synthesizing other azidopurine analogues which might afford important aminyl radicals upon reaction with radiation produced electrons. One example is the $N^{6}$-aminyl radical formed from adenosine, which has been shown in recent years to be integral in the formation of lesions in DNA. ${ }^{124,224}$ Thus, synthesis of 6-azido-2'deoxypurine nucleoside $\mathbf{1 0 1}$ would be advantageous for the study of the adenylyl $N^{6}$ aminyl radical. As in 2-azido purine analogues, the azido group in $\mathbf{1 0 1}$ is thought to generate an aminyl radical through an analogous mechanism as presented in Figure 47 for 89 (Figure 49).

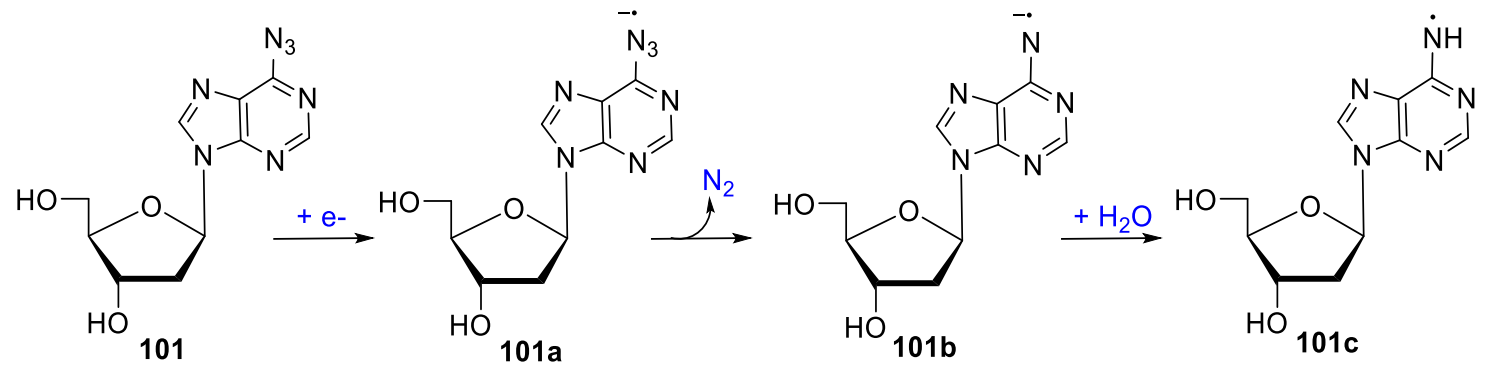

Figure 49. Proposed mechanism of aminyl radical formation from 6-azidopurine 2'deoxynucleoside analogue 
Several methods for the synthesis of 6-azidopurine 2'-deoxynucleoside 101 have been reported in literature. ${ }^{131,140,148}$ Given our previous experience with the synthesis of 6chloropurine nucleoside analogues, we first attempted synthesis of 101 from precursor 104 by replacement of 6-chloro substituent with azide and subsequent deprotection of sugar hydroxyls (Figure 50).
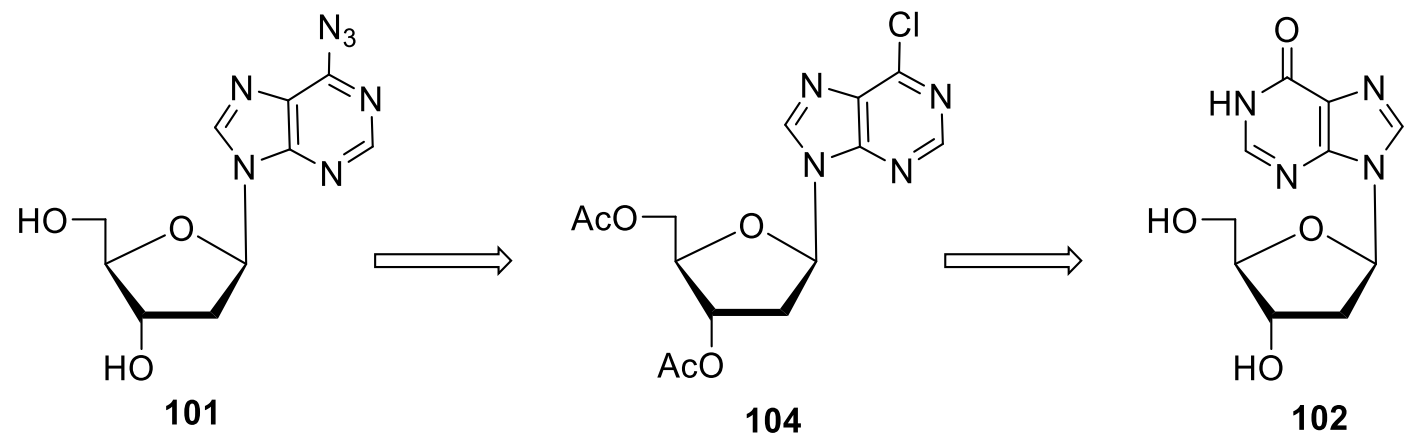

Figure 50. Retrosynthesis of 6-azidopurine 2'-deoxynucleoside from 2'-deoxyinsoine

Synthesis of 6-azidopurine analogue $\mathbf{1 0 1}$ was elaborated by three methods. Method 1 involves the acetylation of 2'-deoxyinosine $\mathbf{1 0 2}$ using $\mathrm{Ac}_{2} \mathrm{O} / \mathrm{DMAP}$ to give $95 \%$ of $\mathbf{1 0 3}$, followed by chlorodeoxygenation with $\mathrm{POCl}_{3}$ to give $\mathbf{1 0 4} .^{211}$ Ultimately, method 1 lead to the minimal formation of product $104(15 \%$, Scheme 42) with major decomposition of substrate $\mathbf{1 0 3}$ observed. We then explored method 2, which involved chlorination of $\mathbf{1 0 3}$ with $\mathrm{SOCl}_{2}$, which also gave $104(45 \%$, Scheme 42$) .{ }^{225}$ Method 2 proved to be more efficient with less decomposition. Method 3, then, employed acetylation of 2'deoxyadenosine $105\left(\mathrm{Ac}_{2} \mathrm{O} / \mathrm{Pyr}, \quad 80 \%\right)$, followed by diazotization using benzyltriethylammonium nitrite (BTEA- $\mathrm{NO}_{2}$ ) and replacement with chloride from TMS$\mathrm{Cl}$ reagent to give less than $10 \%$ of product $\mathbf{1 0 4}$ (as analyzed by TLC). ${ }^{212}$ Method 2 proved to be the most effective for synthesis of $\mathbf{1 0 4}$; despite the moderate yield. Lastly, compound 
104 was treated with $\mathrm{NaN}_{3}$ in $\mathrm{DMF}$ at $50^{\circ} \mathrm{C}$ to afford replacement of chloride by azide giving $55 \%$ of 107.

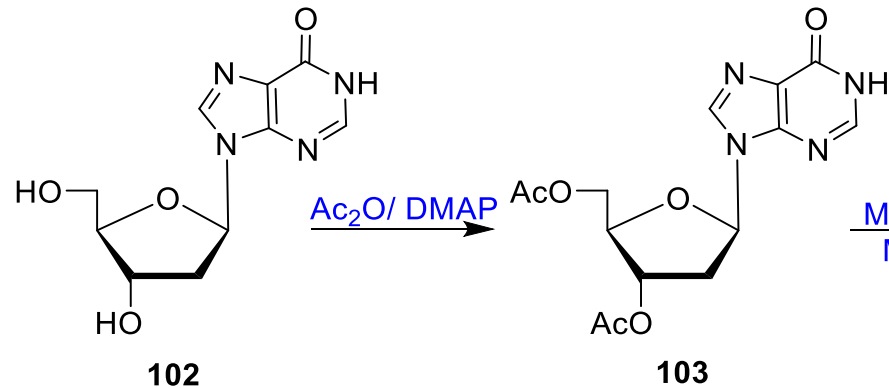
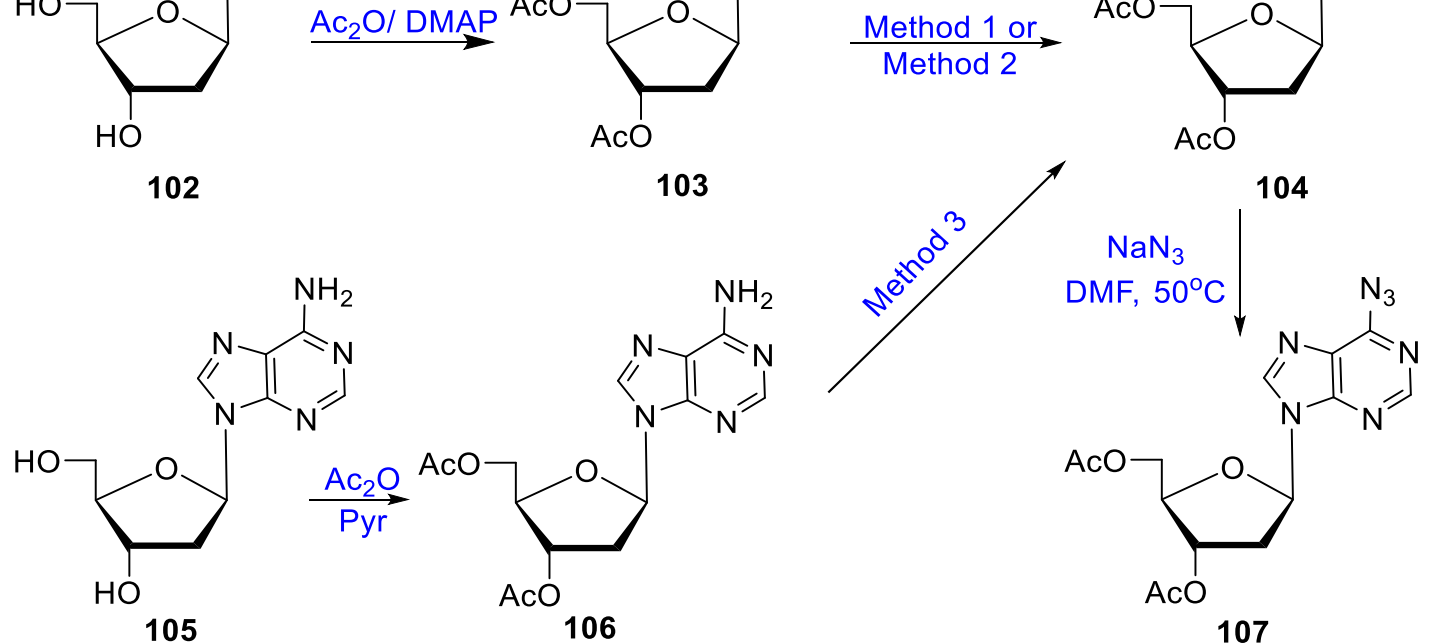

Scheme 42. Routes for the synthesis of 6-azidopurine 2'-deoxynucleoside from 2'deoxyinosine

During proton NMR analysis of $\mathbf{1 0 7}$, two sets of peaks were observed, much like those of 2-azidoadenosine analogues, which highlighted the tautomerization of 107 to $\mathbf{1 0 7 a}$ (Scheme 43). The more deshielded protons were assigned to the tetrazole tautomer.
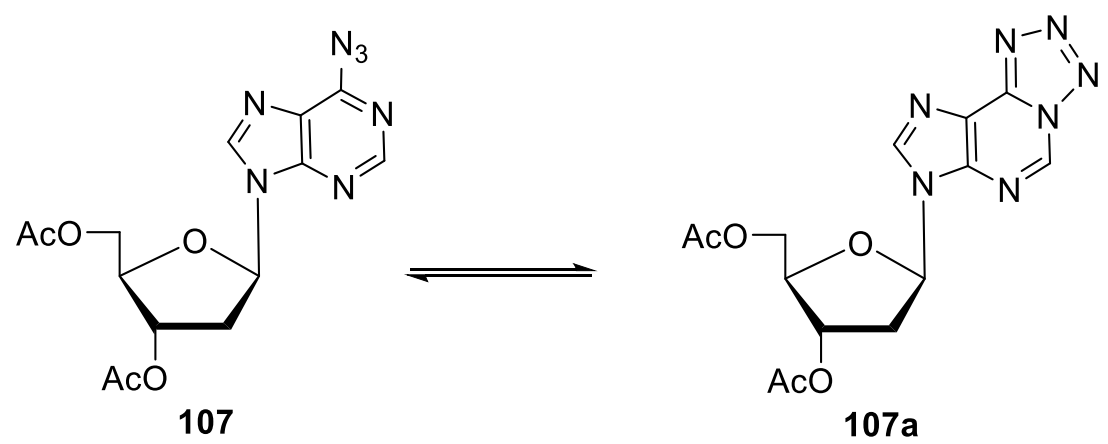

Scheme 43. Tautomerization of 6-azidopurine derivatives 
Deprotection of sugar hydroxyls in $\mathbf{1 0 7}$ was attempted using two methods: 1. with methanolic ammonia, and 2. using methanol saturated with $\mathrm{HCl}$ gas (Scheme 44). Both these methods showed complete decomposition of 107 after only 30 minutes at $0^{\circ} \mathrm{C}$ based on TLC analysis. We decided then to send our protected 6-azidopurine nucleoside analogue $\mathbf{1 0 7}$ to test for aminyl radical formation by EPR.

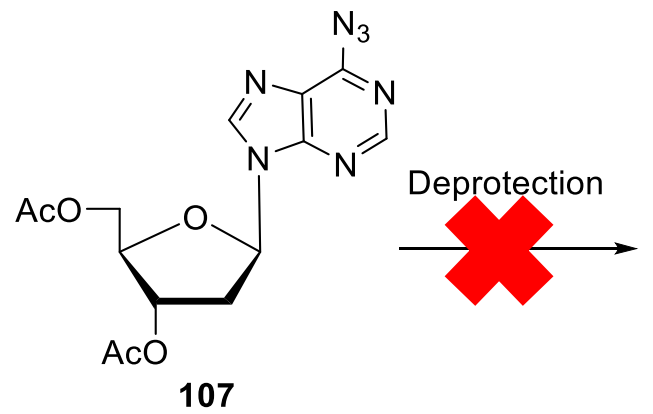

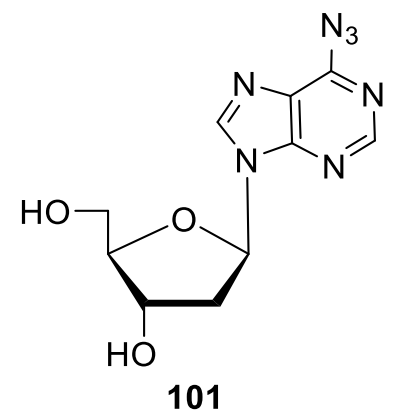

101

Scheme 44. Attempted deacetylation of 6-azidopurine 2'-deoxynucleoside caused complete decompostion

\subsubsection{Synthesis of 4-azidopyrimidine nucleosides to study cytidyl aminyl radical}

Having explored a series of azido modified purine nucleoside analogues, we turned our attention to azido-modified pyrimidine nucleosides. As explained in Section 1.4.3, the formation of a syn-aminyl radical at the $N^{4}$ position of cytidine is a highly important intermediate and precursor to the $\sigma$-iminyl radical that causes damage in double-stranded DNA (Figure 51). As with previous analogues, literature suggests that presence of azide at the 4-position of cytidine can capture electrons and form an aminyl radical. In this case, studies suggest that the aminyl radical 108c may tautomerize to the corresponding iminyl radical 108d. ${ }^{125}$ We therefore targeted synthesis of 4-azidopyrimidine 2'-deoxynucleoside, also named 4-azido-2'-deoxycytidine, $\mathbf{1 0 8}$ to test the aminyl radical formation that might occur in 2'-deoxycytidine in DNA. 


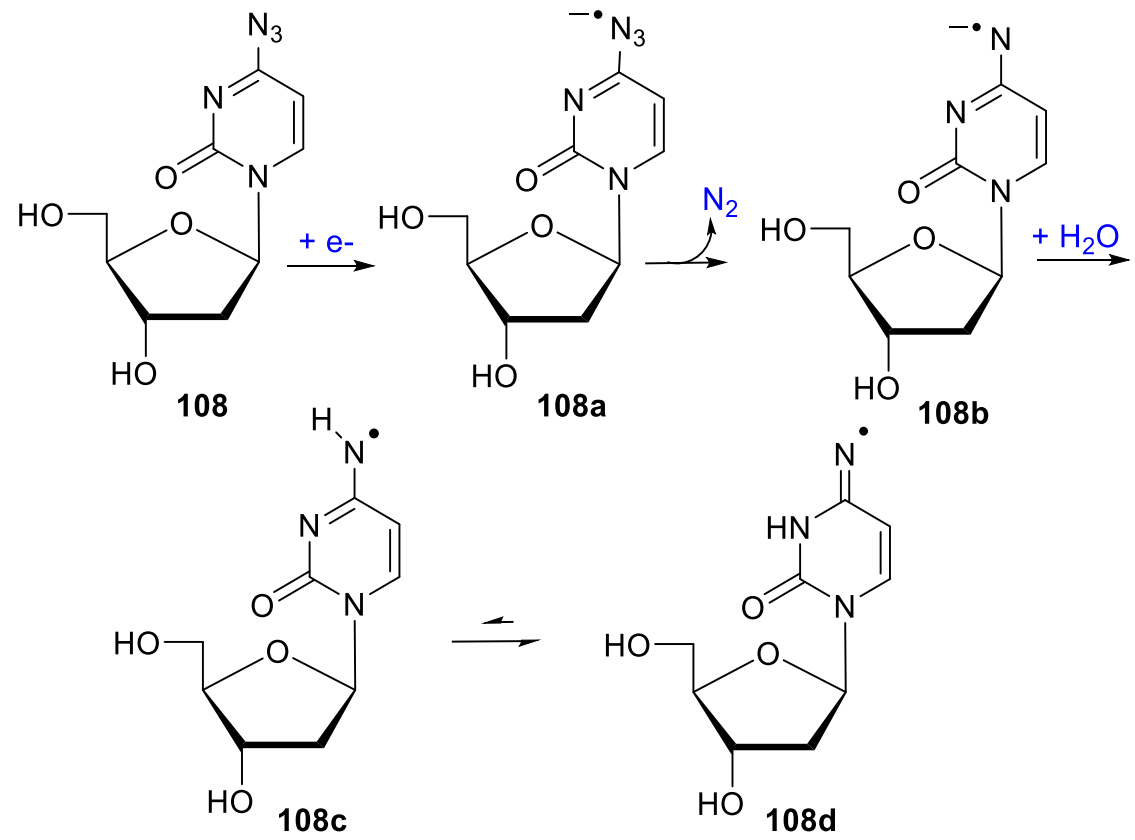

Figure 51. Proposed mechanism for the formation of aminyl radical followed by tautomerization to iminyl radical in 4-azidopyrimidine analogues

The synthesis of 4-azidopyridimidine analogues has been previously published, ${ }^{129,151}$ utilizing Divakar \& Reese protocols ${ }^{154}$ via elaboration of 1,2,4-triazole group at the 4position of pyrimidine ring as a leaving group. This triazole group can then be replaced with an azide anion from $\mathrm{NaN}_{3}$ or $\mathrm{LiN}_{3}$ in organic solvent. Figure 52 shows the retrosynthetic approach for formation of 4-azidopyridimidine analogue $\mathbf{1 0 8 .}$

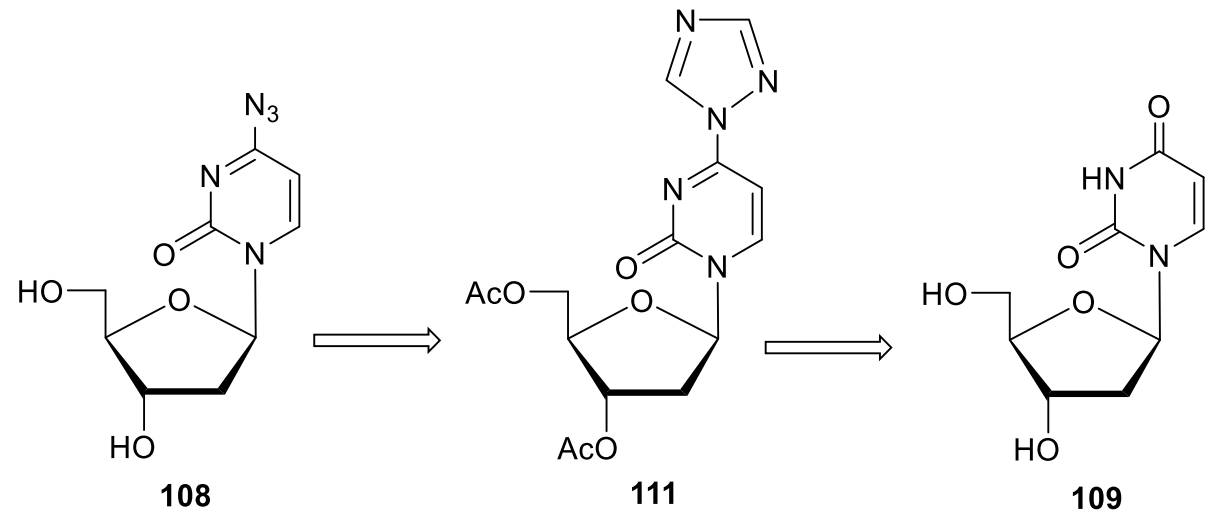

Figure 52. Retrosynthesis for formation of 4-azidopyridimidine from 2'-deoxyuridine 
Synthesis of 4-azido-2'-deoxypyrimidine nucleoside 108 started with acetylation of the sugar hydroxyls of 2'-deoxyuridine $\mathbf{1 0 9}$ with acetic anhydride/DMAP to give protected 2'deoxyuridine 110 (90\%; Scheme 45). Treatment of 110 with $\mathrm{POCl}_{3}$ followed by substitution with 1,2,4-triazole gave 111 in 75\% yield. Replacement of triazole moiety with azide proved to be more complicated than previously expected. This reaction was attempted by three methods. Method 1 involves utilizing $\mathrm{NaN}_{3}$ in DMF solvent. This method proved unsuccessful even upon heating as high as $100^{\circ} \mathrm{C}$ and did not yield product 112 (as observed by TLC analysis). Solubility was thought to be a problem with $\mathrm{NaN}_{3}$ reagent, and thus, method 2 utilized $\mathrm{LiN}_{3}$ generated in situ from LiF and TMS-N $\mathrm{N}_{3}$ in tetramethylethylenediamine was attempted. ${ }^{129}$ The more covalent nature of the lithiumazide bond, when compared to the sodium azide bond, was expected to solubilize better in organic solvents. The high temperature used for this reaction $\left(105-110^{\circ} \mathrm{C}\right)$ caused some decomposition but gave moderate (40\%) yield of product 112. Method 3 proved to the most successful, with $85 \%$ yield of $\mathbf{1 1 2}$, by utilizing acetic acid in order to protonate 1,2,4-triazole moiety to afford a better leaving group. A combination of solvent $\left[\mathrm{H}_{2} \mathrm{O} / \mathrm{DMF}\right.$ (1:4)] was used to afford better solubility of the $\mathrm{NaN}_{3}$ reagent. 


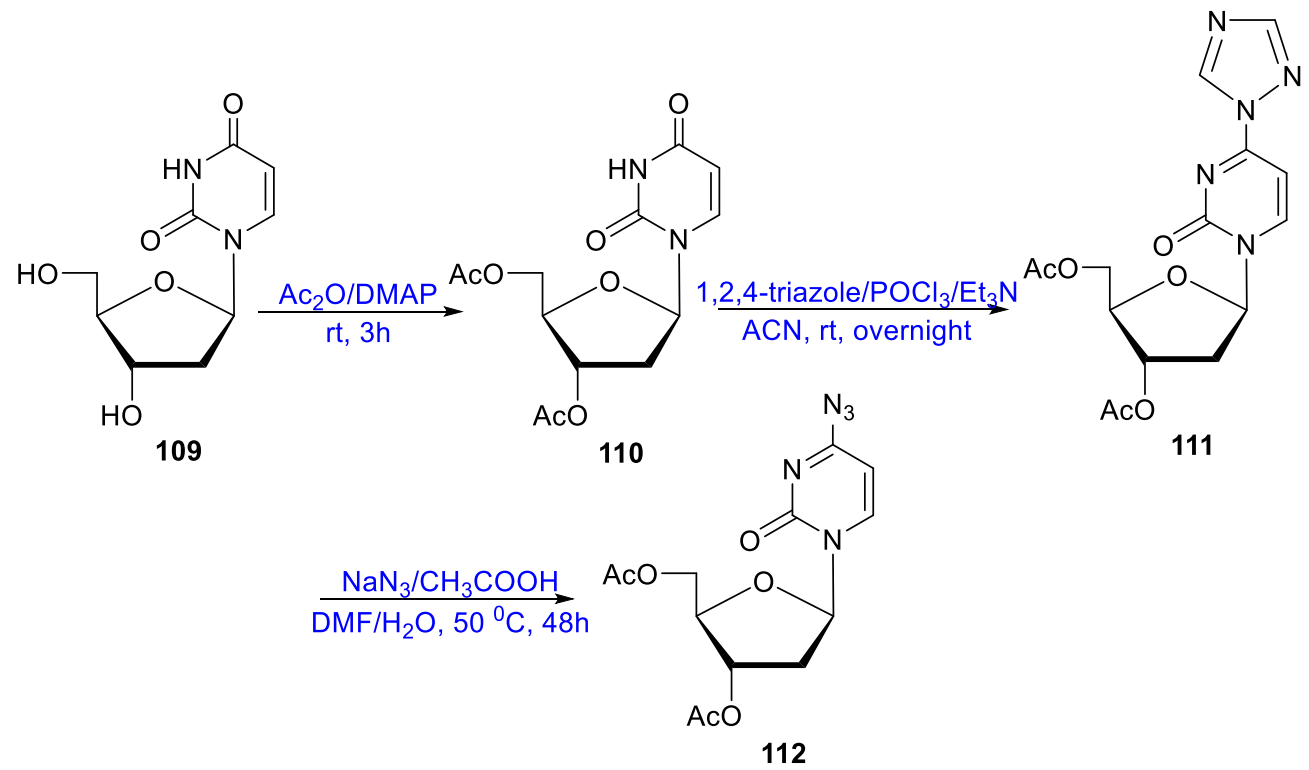

Scheme 45. Synthesis of 4-azidopyrimidine 2'-deoxy nucleoside

\subsubsection{Bicyclic tetrazole analogues}

Attempted deprotection of $\mathbf{1 1 2}$ with methanolic ammonia, $\mathrm{NaOMe}$, and $\mathrm{NaCN} / \mathrm{ethanol}$ yielded decomposition products as reported by Kotra et al. ${ }^{151}$ However, deprotection using methanol saturated with $\mathrm{HCl}$ was found to give deacetylated 4-tetrazolo product $\mathbf{1 1 3}$ quantitatively $^{128}$ (Scheme 46). Identification by UV absorption showed the protected 4azido 112 had $\lambda_{\max }=255 \mathrm{~nm}$ and $265 \mathrm{~nm}$, while deprotected 4-tetrazolo 113 was had $\lambda_{\max }=$ $257 \mathrm{~nm}$ and $267 \mathrm{~nm}$. Furthermore, NMR analysis of 112 and 113 showed that peaks for $\mathrm{H} 5, \mathrm{H} 6$, and H1' were much more deshielded in 113 than in 112, which would suggest formation of tetrazole tautomer. Presence of azide functionality in $\mathbf{1 1 2}$ was determined by performing a strain-promoted click-reaction using a cyclooctyne analogue on TLC, which showed conversion ( $60 \%)$ to a "click" higher moving product. Given these finding, we decided to test formation of aminyl radical on protected 112, and tetrazolo 113. 

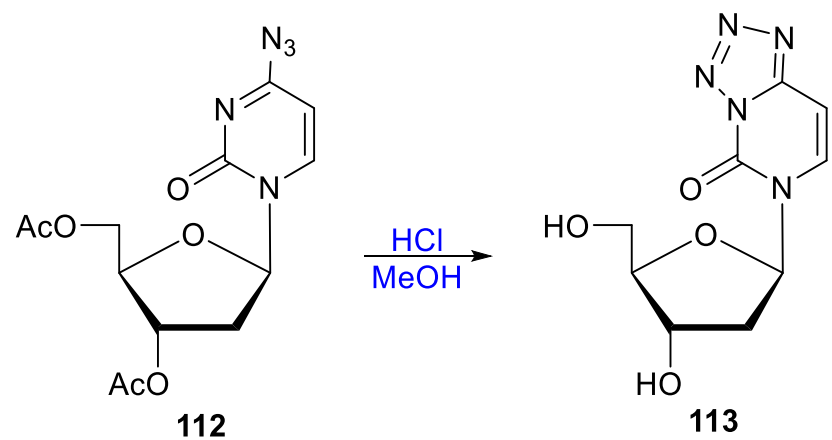

Scheme 46. Deprotection of 3',5'-di- $O$-acetyl protected 4-azidopyrimidine nucleoside giving 4-tetrazolopyrimidine nucleoside product

We also explored synthesis of ribose counterpart of the 4-azido-2'-deoxyuridine 112, and 4-tetrazolo-2'-deoxyuridine 113 to test for possible differences in the radical formation during irradiation. Much like the synthesis of 112 and 113, synthesis of protected 4azidouridine 114 and 4-tetrazolouridine nucleoside 115 was achieved through addition of 1,2,4-triazole at the 4 position, followed by replacement with azide. Thus, uridine $\mathbf{1 1 6}$ was treated with acetic anhydride and DMAP to yield tri-acetylated uridine 117 (90\%; Scheme 47). Then, compound 117 was treated with $\mathrm{POCl}_{3}, \mathrm{Et}_{3} \mathrm{~N}$, and 1,2,4-triazole to form 4triazole substituted derivative 118 in roughly $78 \%$ yield. Subsequent treatment with acetic acid/ $\mathrm{NaN}_{3}$ in $\mathrm{DMF} / \mathrm{H}_{2} \mathrm{O}$ afforded protected 4-azidouridine 114 (80\%). Given our experience with decomposition during deprotection of 4-azido-2'-deoxyuridine analogue, 114 was simply treated with $\mathrm{MeOH} / \mathrm{HCl}$ to form 4-tetrazolo derivative $\mathbf{1 1 5}$ in $90 \%$ yield. Both compounds 114 and 115 were sent to collaborators to test for aminyl radical formation and comparison with 2'-deoxy analogues. 


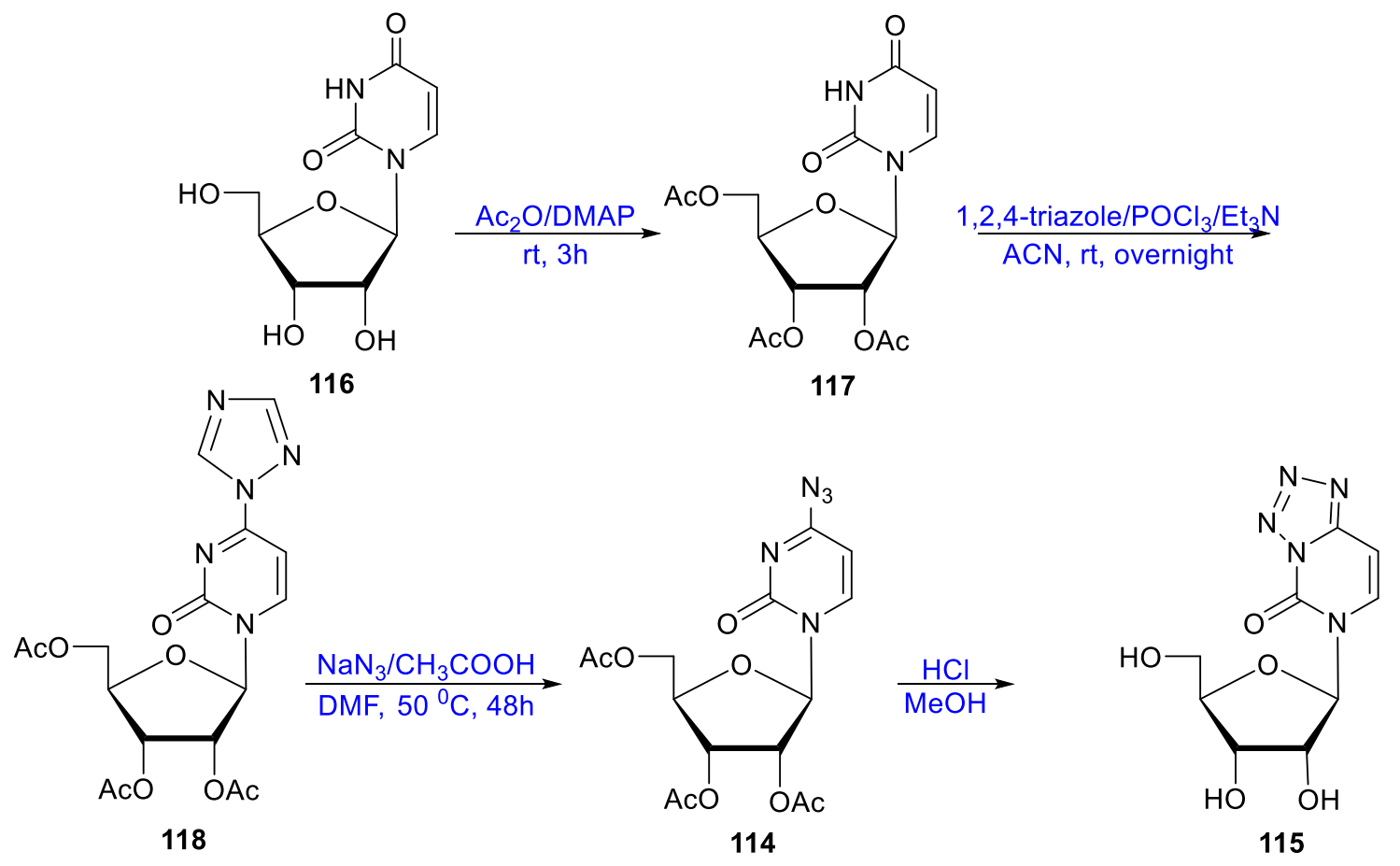

Scheme 47. Synthesis of 4-azidopyrimidine and 4-tetrazolopyrimidine nucleosides

\subsubsection{ESR studies of radicals generated from azido-modified nucleosides}

\subsubsection{From azido-modified pyrimidine nucleosides}

Radiation-produced electron addition to the protected 4-azido-2'-deoxyuridine 112 and triazole compound $\mathbf{1 1 3}$ (concentration $=2-3 \mathrm{mg} / \mathrm{mL}$ ) allowed to identify novel radicals. ESR spectra (absorbed dose $=600 \mathrm{~Gy}$ at $77 \mathrm{~K}$; homogeneous glassy or supercooled homogeneous 7.5 M LiCl/ $\mathrm{D}_{2} \mathrm{O}$ solutions) were recorded at $77 \mathrm{~K}$ just after irradiation and subsequently by gradually warming these samples up to $145 \mathrm{~K}$ (Figure 53). ${ }^{226} \mathrm{It}$ is evident from the ESR spectra that the center of the spectra, lineshape and overall hyperfine splitting (i.e., total spectral width) did not change before (i.e., at $77 \mathrm{~K}$ ) and after warming (i.e., at $145 \mathrm{~K}$ ). Figure 53 is matched with a simulated spectrum (black line, Figure 53, Panel B). Simulated spectrum has been obtained employing a mixed (Lorentzian/Gaussian=10) line shape with two axially symmetric anisotropic ${ }^{14} \mathrm{~N}$ (nuclear spin $=1$ ) hyperfine coupling 
constant (HFCC) values of $(22.0,0,0) \mathrm{G}$ and $(20,0,0) \mathrm{G}, g_{z z}, g_{x x}, g_{y y}(2.0017,2.0041$, 2.0041) along with an isotropic Gaussian line-width of 6 G. Even the 77 K ESR spectrum obtained after subsequent photoexcitation of sample 112 at $145 \mathrm{~K}$ for $45 \mathrm{~min}$ remains unchanged. These results establish that the same radical is formed via reaction of radiationproduced prehydrated electron in both compounds.

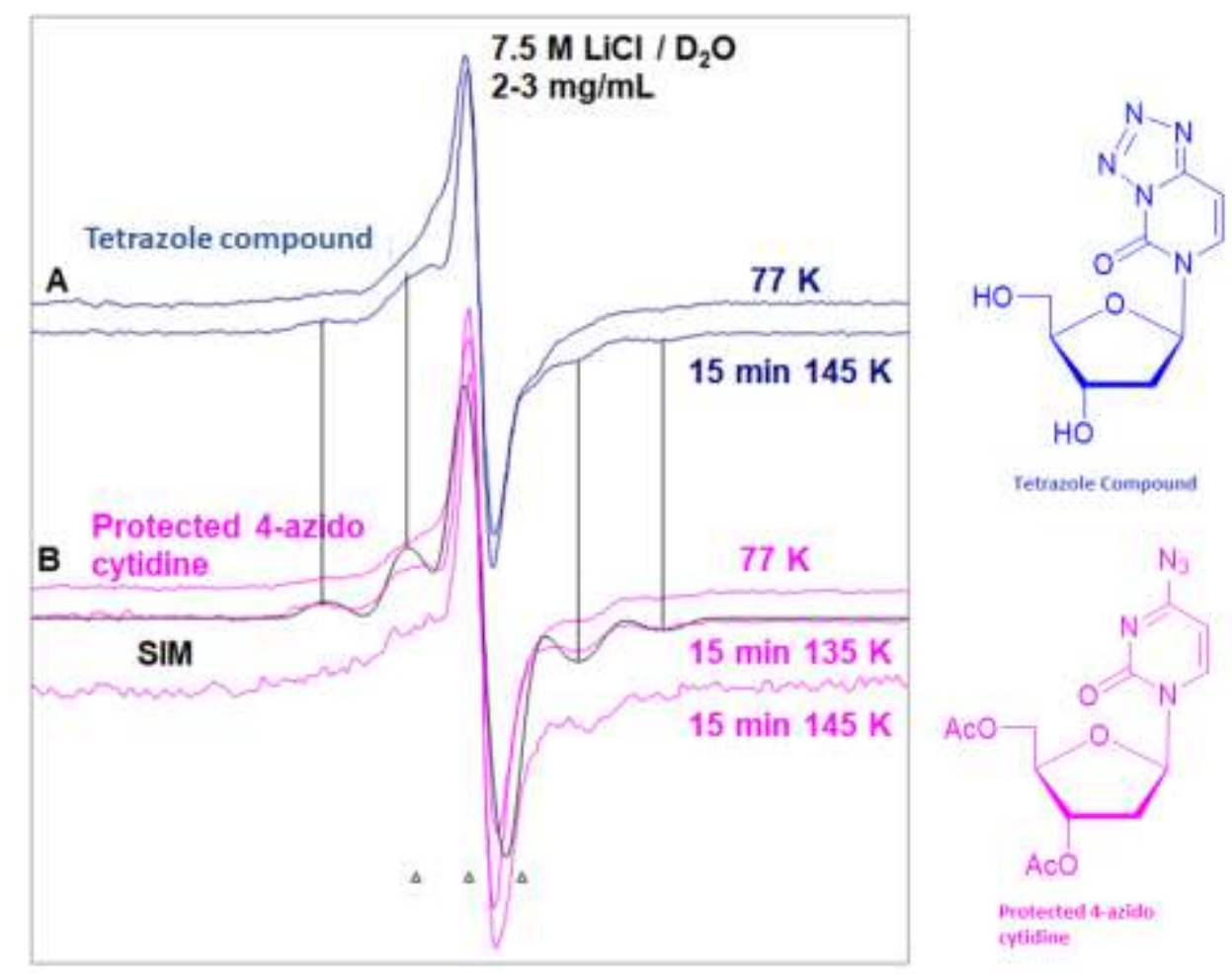

ESR spectra obtained from matched homogeneous glassy samples $(2-3 \mathrm{mg} / \mathrm{ml}$ in $7.5 \mathrm{M}$ $\mathrm{LiCl} / \mathrm{D}_{2} \mathrm{O}(\mathrm{pH} / \mathrm{pD}$ ca. 5$)$ of tetrazole $(\mathbf{1 1 3}$, blue) and of protected 4-azidocytidine (112, pink). The first spectrum in panel (A) and in panel (B) shows $\mathrm{RN}_{3}{ }^{\circ}$ formation via radiationproduced (absorbed dose $=600 \mathrm{~Gy}$ ) prehydrated electron attachment at $77 \mathrm{~K}$ in the dark. Second set of spectra in panel (A) and in panel (B) were obtained after warming or annealing the samples for $15 \mathrm{~min}$ in the dark at $135 \mathrm{~K}$ and at $145 \mathrm{~K}$. The simulated spectrum (black) is shown in panel (B) and for simulation parameters, consult text. Similarities of the $A_{z z}$ HFCC in the spectra of both compounds were shown by vertical solid lines.

Figure 53. ESR spectra obtained from compounds 112 and 113 via irradiation with radiation-produced prehydrated electrons. 
Theoretical calculations (Figures 54 and 55) propose a particular conformation of the observed radical which give rise to two high anisotropic N-couplings $\left(\boldsymbol{A}_{z z}=\right.$ ca. $27 \mathrm{G}$ and $\left.34 \mathrm{G}, \boldsymbol{A}_{\boldsymbol{x} x}=\boldsymbol{A}_{\boldsymbol{y} y}=0\right)$ compared to the experimentally observed $\boldsymbol{A}_{z z}$ values of aniosotropic Ncouplings $\left(\boldsymbol{A}_{z z}=22 \mathrm{G}\right.$, and $20 \mathrm{G}, \boldsymbol{A}_{\boldsymbol{x} x}=\boldsymbol{A}_{\boldsymbol{y} \boldsymbol{y}}=0$, vide supra $)$ for irradiation of $\mathbf{1 1 2}$ and $\mathbf{1 1 3}$ (Figure 53). Moreover, these spectra are different from the reported spectrum of $\pi$-type neutral aminyl radical $(\mathrm{C}(\mathrm{N} 4-\mathrm{H}) \bullet)$ from 1-methylcytosine. ${ }^{69}$ Thus, we assign these spectra to the azido anion radical $\left(\mathrm{RN}_{3}{ }^{\circ}\right)$. These ESR spectral results show that (a) in tetrazole, $\mathrm{RN}_{3}{ }^{\circ-}$ formation occurs via dissociative electron attachment, where the radiation-produced electron attaches to the tetrazole ring at $77 \mathrm{~K}$ and is followed by breakage of the cytosine ring N3-azido bond in the anion radical (Scheme 48), and (b) under the experimental conditions of ESR spectroscopic investigations [homogeneous glassy solutions at low temperatures $(77 \mathrm{~K}$ to $145 \mathrm{~K})$ ] $\mathrm{RN}_{3}{ }^{\bullet-}$ from these compounds do not get converted to the expected neutral aminyl radical, $\mathrm{C}(\mathrm{N} 4-\mathrm{H}) \bullet($ radical 108c in Figure 51) in the ground state as expected from earlier studies with azido-DNA-models. ${ }^{104-106}$

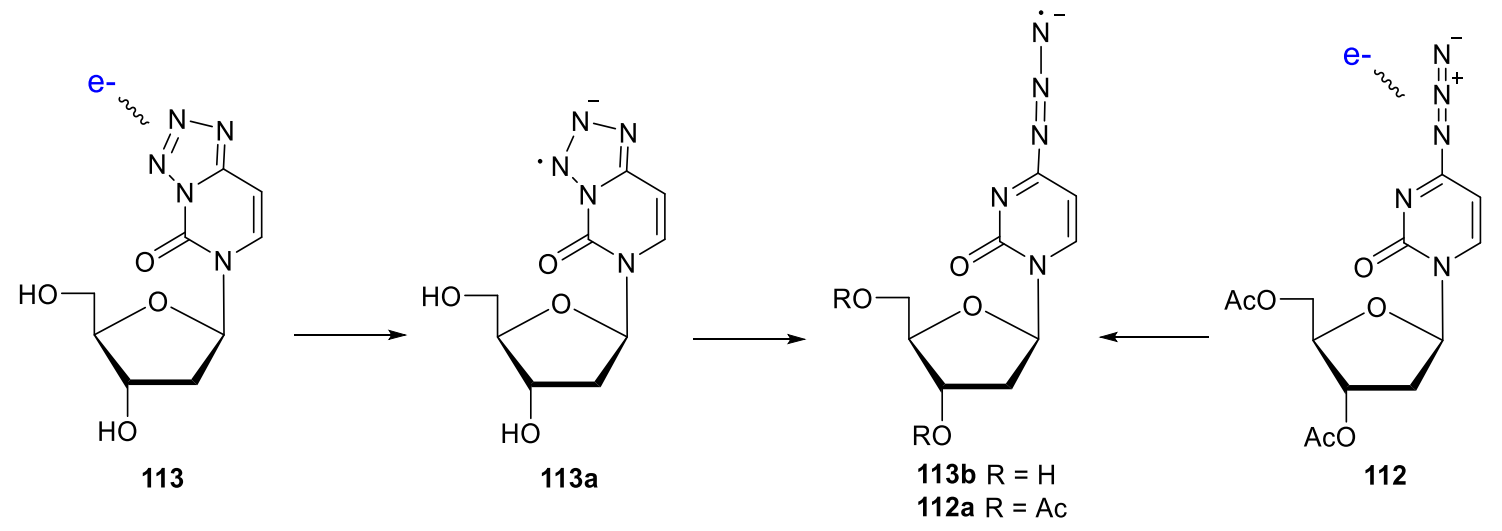

Scheme 48. Reaction of tetrazole 113 and azido 112 with radiation produced electrons to form RN3•-. 

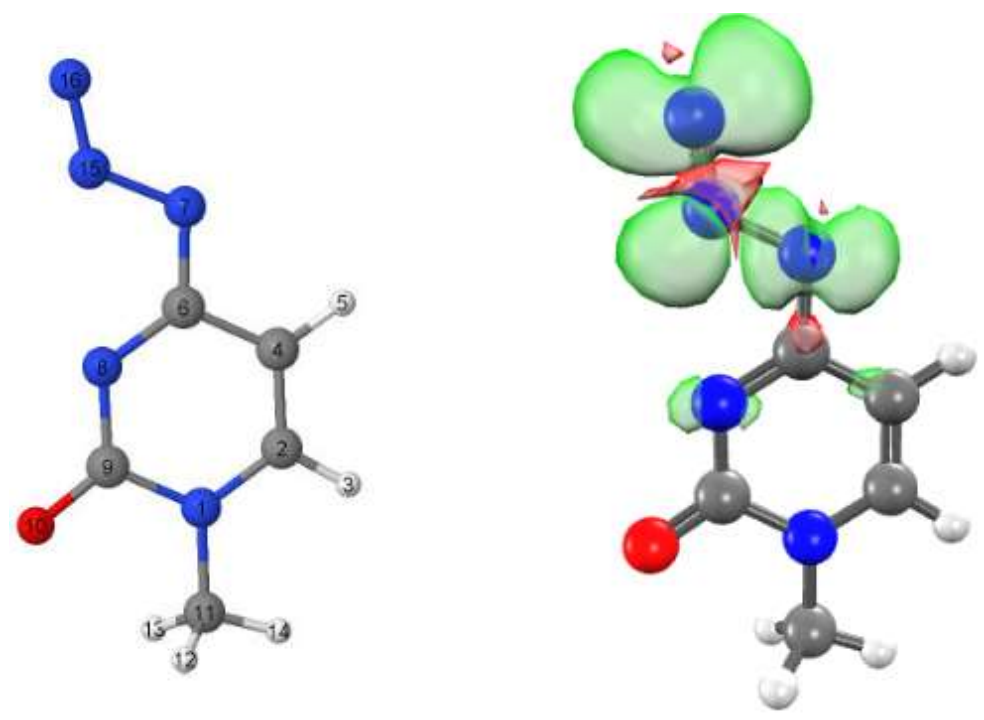

The azido anion radical (RN3•-) in 1-methylcytosine was geometry-optimized employing DFT/B3LYP/6-31G** method. Spin density distributions and hyperfine coupling constants were calculated considering full solvation (IEF-PCM) under Gaussian'09 suit of program.

Figure 54. Theoretical calculations of azide anion radical (RN3•-) in 1-methylcytosine.
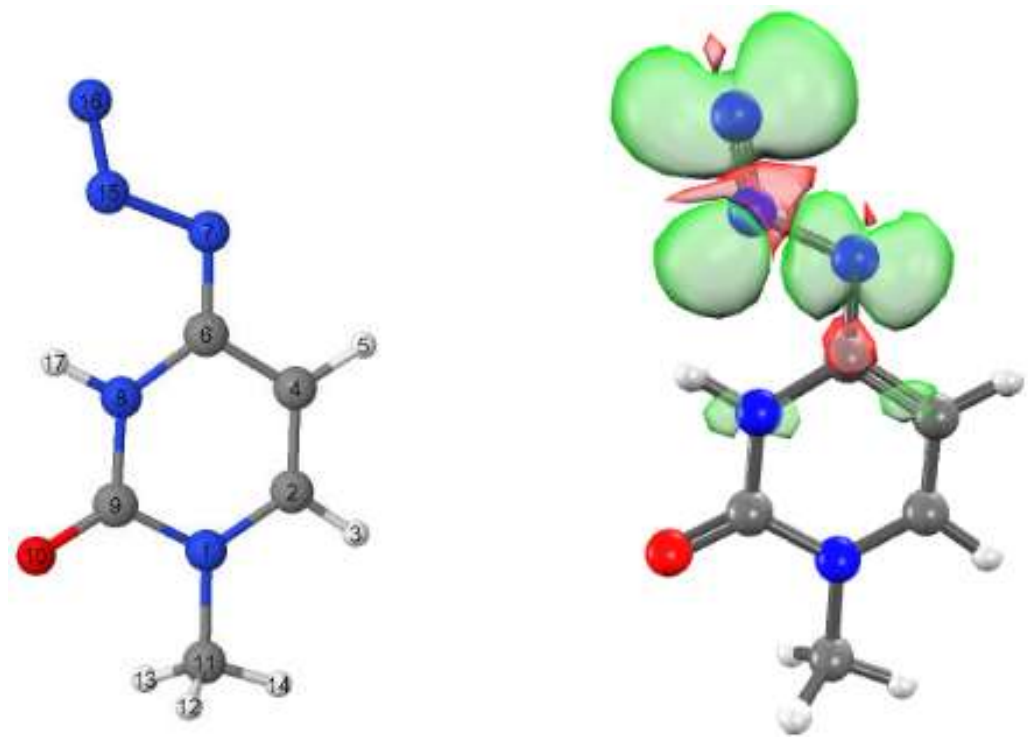

The azido anion radical $\left(\mathrm{RN}_{3}{ }^{-}\right)$in $\mathrm{N} 3$-protonated 1-methylcytosine was geometryoptimized employing DFT/B3LYP/6-31G** method. Spin density distributions and hyperfine coupling constants were calculated considering full solvation (IEF-PCM) under Gaussian'09 suit of program.

Figure 55. Theoretical calculations of azide anion radical (RN3•-) in N3-protonated 1methylcytosine. 


\subsection{Proposed conversion $\mathrm{RN}_{3}{ }^{\bullet}$ to $\mathrm{C}^{\prime} \bullet$ sugar radical via $\mathrm{UV}$}

\section{photoexcitation}

UV-photoexcitation of the $\mathrm{RN}_{3}{ }^{\circ}$, produced from tetrazole 113 at $77 \mathrm{~K}$, for 14 min led to the formation of ca. $20 \%$ conversion to $\mathrm{C}^{\prime} \cdot$ (Figure 56), due to similarities of the anisotropic doublet from anisotropic alpha-proton at C5'. Overall spectral width of this anisotropic doublet; however, is ca. $14.5 \mathrm{G}$ for $\mathbf{1 1 3}$ (center of black spectrum) versus ca. $19 \mathrm{G}$ of the authentic $\mathrm{C}^{\prime} \cdot$ produced via photoexcitation of guanine cation radical in $\mathrm{dGuo}^{227}$ (red spectrum). Owning to a number of possible conformation of $\mathrm{C}^{\prime} \cdot$, the orientation of the radical site p-orbital at $\mathrm{C}^{\prime}$ with respect to the anisotropic alpha $\mathrm{C} 5$ '-H proton can change and as a result, HFCC value of the anisotropic alpha C5'-H proton can vary. ${ }^{228}$ This could be attributed to the conversion of $\mathrm{RN}_{3}{ }^{-}$to the neutral aminyl radical in the excited state and subsequent formation of the sugar radical via either intramolecular or intermolecular $\mathrm{H}$-atom abstraction reaction from $\mathrm{C} 5$ ' by the excited aminyl radical (Scheme 49). It is established in literature, that the excited aminyl radicals from one-electron oxidized pyrimidines and purines produce sugar radicals. ${ }^{94,227,228}$ Due to the experimental condition (i.e., photoexcitation at $77 \mathrm{~K}$ of the homogeneous glassy system), it is proposed that the $\mathrm{H}$-atom abstraction reaction should occur via a fast proton-coupled electron transfer process. These results further establish that, $\mathrm{RN}_{3}{ }^{\circ}$ from 112 or from 113 are found to be stable and hence not reactive in comparison to the reactivities of neutral aminyl radicals that were produced via electron addition to azides which are either in the sugar moiety ${ }^{104,105}$ or in the base as azidomethyl or azidovinyl. ${ }^{106}$ 


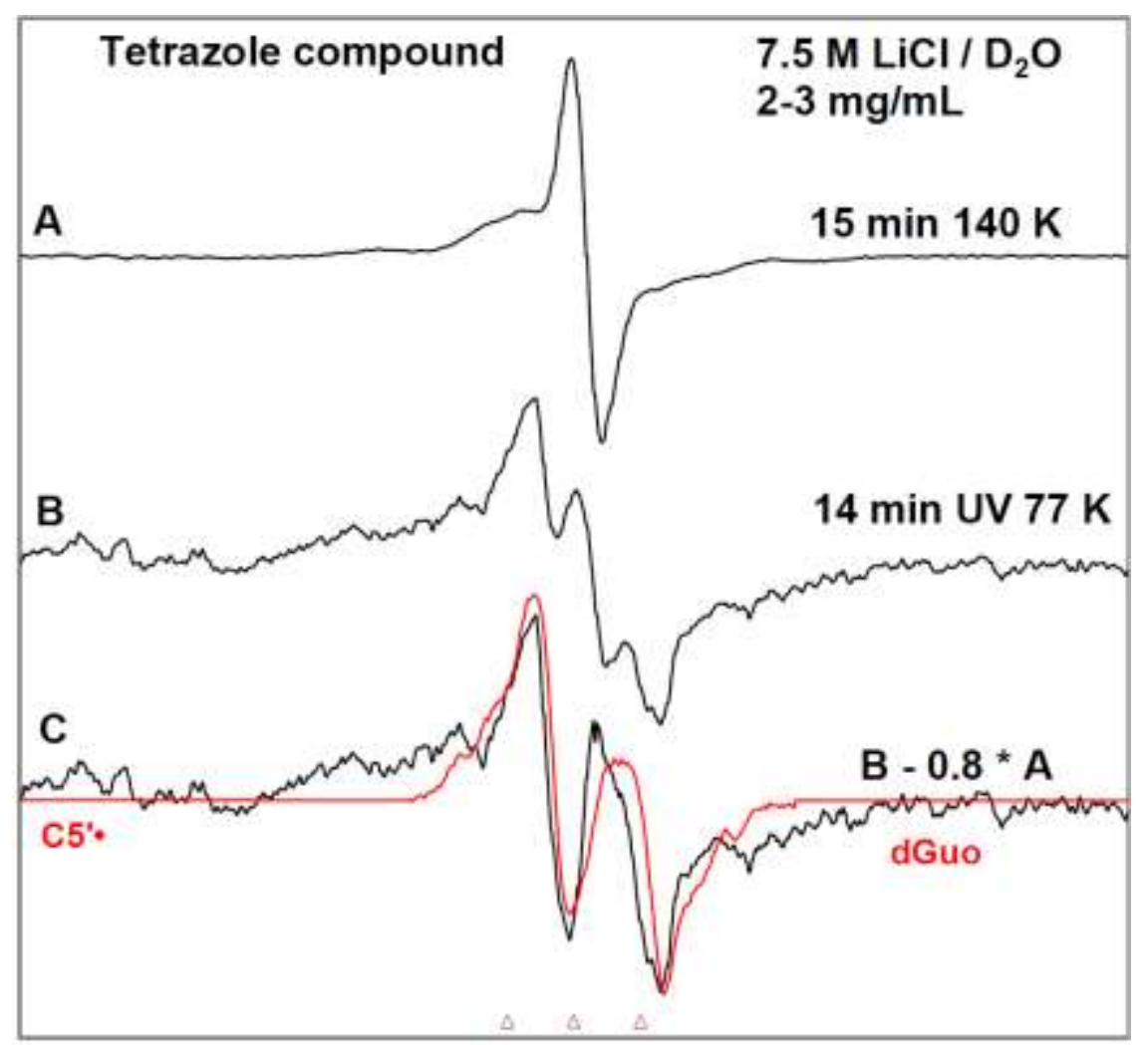

The first spectrum (panel A) is obtained after $\gamma$-irradiation of tetrazole 113 (absorbed dose $=600 \mathrm{~Gy}$ ) at $77 \mathrm{~K}$ in the dark followed by annealing for $15 \mathrm{~min}$ in the dark at $140 \mathrm{~K}$. (B) Spectrum of this sample was obtained at $77 \mathrm{~K}$ after UV-radiation at $77 \mathrm{~K}$ for $14 \mathrm{~min}$. (C) The black spectrum is obtained after $80 \%$ subtraction of spectrum (A) from spectrum (B). The red spectrum is that of $\mathrm{C}^{\prime} \bullet$, produced from $\mathrm{G}^{{ }^{+}}$in 8 -D-3'-dGMP via photoexcitation. ${ }^{227}$ All spectra were recorded at $77 \mathrm{~K}$.

Figure 56. ESR spectra obtained after $\gamma$-irradiation and subsequent UV photoexcitation of the tetrazole 113. 


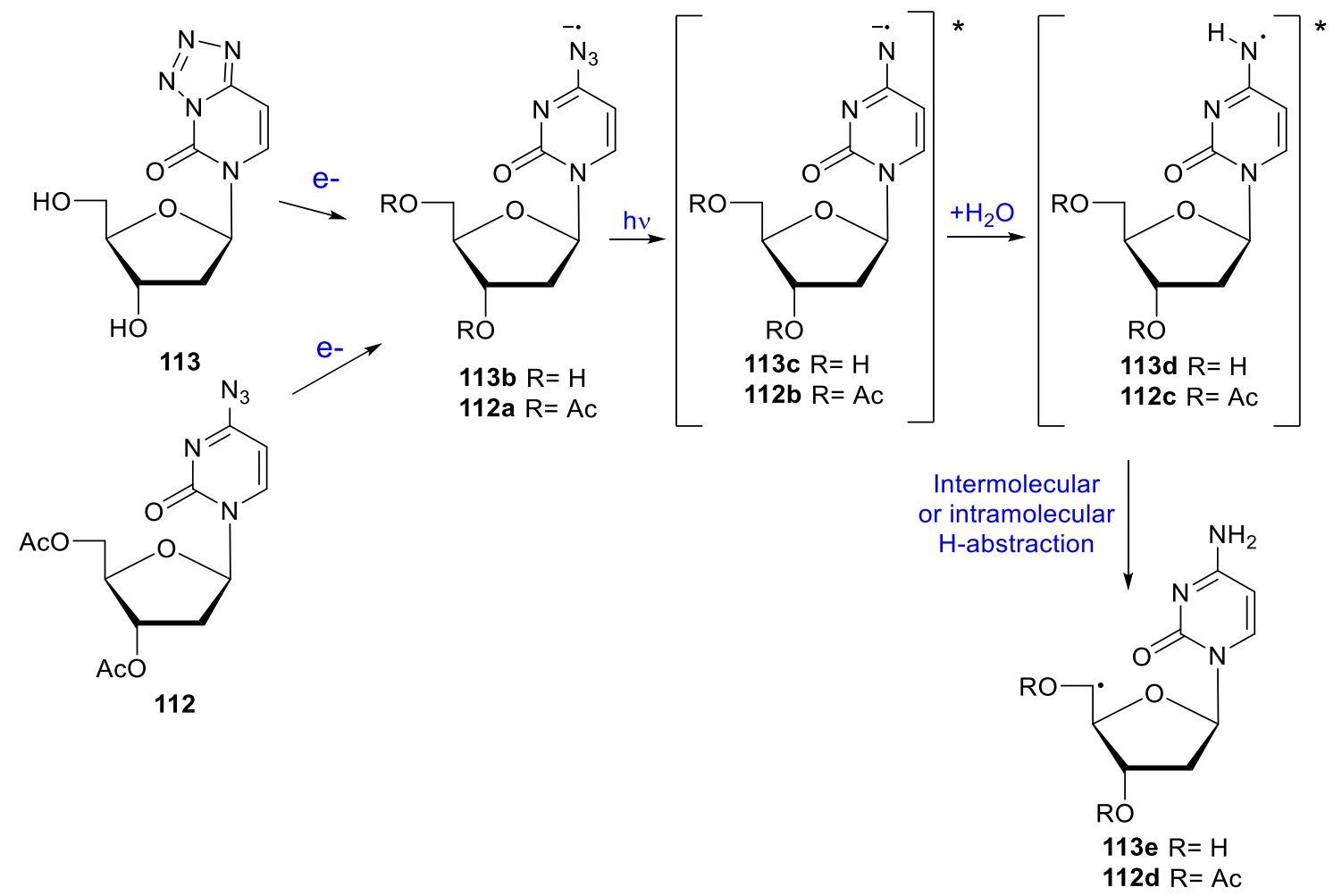

Scheme 49. Proposed mechanism for the formation of C5'-sugar radical upon photoexcitation of the $\mathrm{RN}_{3}{ }^{-}$in tetrazole 113 and azido 112.

We then ran samples from commercially available 5-azidouridine (5-AzU) to check whether the unexpected formation of the azide anion radical would also occur upon radiation-produced electron addition to 5-AzU. The ESR spectral results are presented in Figure 57. 


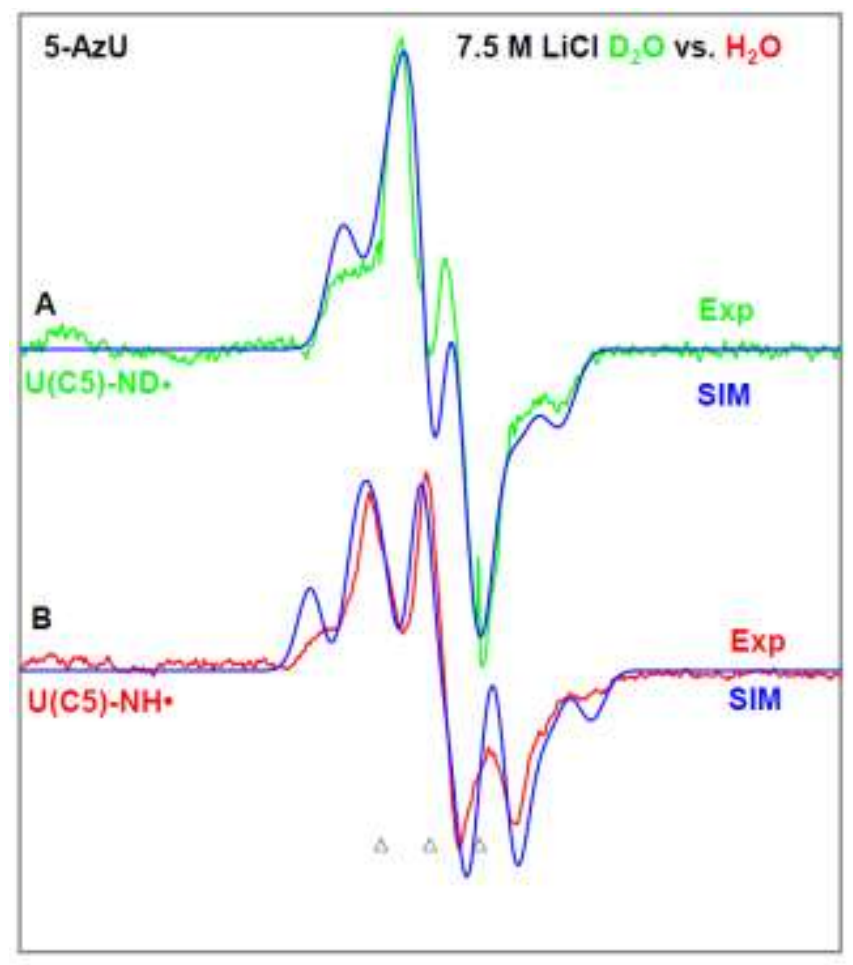

$77 \mathrm{~K}$ ESR spectrum of the aminyl radical (U(C5)-ND•/(U(C5)-NH•)) formed via radiationproduced one-electron attachment (absorbed dose $=600 \mathrm{~Gy}$ at $77 \mathrm{~K}$ ) to 5-AzU (Panel A) (U(C5)-ND•) (green) in $7.5 \mathrm{M} \mathrm{LiCl}(\mathrm{D} 2 \mathrm{O})$, (Panel B) (U(C5)-NH•) (red color) in $7.5 \mathrm{M}$ $\mathrm{LiCl}\left(\mathrm{H}_{2} \mathrm{O}\right)$. The simulated spectra (blue) (for simulation ESR parameters, see text) are superimposed on the top of the experimentally recorded spectrum. Comparison of the experimentally recorded spectra with those obtained via simulation clearly show that the $A_{z z}$ component ca. $17 \mathrm{G}$ due to the $\alpha-\mathrm{N}-\mathrm{H}$ proton of the aminyl radical in $\mathrm{H}_{2} \mathrm{O}$ (U(C5)-NH•) (red color) is lost in the $\mathrm{D}_{2} \mathrm{O}$ glasses (green color). All spectra were recorded at $77 \mathrm{~K}$ and the line components due to $\mathrm{Cl}_{2} \bullet^{-}$are subtracted from these recorded spectra.

Figure 57. ESR spectrum of aminyl radical formed upon radiation-produced electron addition to 5-azidouridine (5-AzU)

In Figure 57 Panel A, the ESR spectrum (green) of the aminyl radical (U(C5)-ND•) from 5-AzU in $\mathrm{D}_{2} \mathrm{O}(7.5 \mathrm{M} \mathrm{LiCl})$, recorded at $77 \mathrm{~K}$ and after subtraction of the line components from $\mathrm{Cl}_{2} \bullet^{-}$, is shown. In Figure 57 Panel B, ESR spectrum of the aminyl radical (U(C5)-NH•) (red) is obtained under identical conditions from 5-AzU in $\mathrm{H}_{2} \mathrm{O}(7.5 \mathrm{M} \mathrm{LiCl})$.

Comparison of widths of these two spectra clearly shows that an additional $17 \mathrm{G}$ proton hyperfine coupling in the aminyl radical in $\mathrm{H}_{2} \mathrm{O}\left(\mathrm{U}(\mathrm{C} 5)-\mathrm{NH} \bullet\right.$ ) (red) is present in the $\mathrm{H}_{2} \mathrm{O}$ glasses and is missing in the $\mathrm{D}_{2} \mathrm{O}$ glasses (green). These results show that the source of the 
proton in the formation of the aminyl radical ( $(\mathrm{C} 5)-\mathrm{NH} \bullet$ ) via protonation of the nitrene anion radical $\left.\left(\mathrm{U}(\mathrm{C} 5)-\mathrm{N}^{-}\right)\right)$, is the solvent $\left(\mathrm{H}_{2} \mathrm{O}\right)$. This additional hyperfine coupling of 17 $\mathrm{G}$ is the $\boldsymbol{A}_{z z}$ component of the N-H $(\alpha-\mathrm{H})$ coupling that contributes to the outer line components and this component adds to the $\boldsymbol{A}_{z z}$ component of the anisotropic hyperfine coupling of the $\mathrm{N}$-atom of $\mathrm{U}(\mathrm{C} 5)-\mathrm{NH} \bullet$. Thus, from the experimentally recorded spectrum (red) of $\mathrm{U}(\mathrm{C} 5)-\mathrm{NH} \bullet$ (Figure 57 Panel B), only the $\boldsymbol{A}_{z z}$ component of the $\mathrm{N}-\mathrm{H}(\alpha-\mathrm{H})$ hyperfine coupling could be determined by experiment.

The spectrum of U(C5)-ND• in Figure 57 Panel A and that of the U(C5)-NH• in Figure 57 Panel B have been simulated (blue). For U(C5)-ND•, the simulation parameters are: C6H HFCC $(14.5,6.0,11.0) \mathrm{G}$, radical site anisotropic axially symmetric N HFCC $(0,0,23$ G) G, N-D (for the exchangeable $\alpha-\mathrm{H}=4.0,1.0,2.5) \mathrm{G}, g_{x x}, g_{y y}, g_{z z}=(2.0043,2.0043$, 2.0020), a mixed (Lorentzian/Gaussian=1.0) lineshape, and an isotropic linewidth of 7 G. For $\mathrm{U}(\mathrm{C} 5)-\mathrm{NH} \bullet$, the simulation parameters are: $\mathrm{C} 6-\mathrm{H} \operatorname{HFCC}(17.0,6.0,13.6) \mathrm{G}$, radical site anisotropic axially symmetric N $\operatorname{HFCC}(0,0,20 \mathrm{G}) \mathrm{G}, \mathrm{N}-\mathrm{H}$ (for the exchangeable $\alpha$ $\mathrm{H}=25.0,6.0,17.0) \mathrm{G}, \quad g_{x x}, \quad g_{y y}, \quad g_{z z}=(2.0043,2.0043,2.0020)$, a mixed (Lorentzian/Gaussian=1.0) lineshape, and an isotropic linewidth of $9 \mathrm{G}$. The simulated spectra match quite well with that of the experimentally observed spectra.

In 5-AzU, the azido group is at meta position with respect to the $\mathrm{N} 1$ and $\mathrm{N} 3$ ring nitrogens; on the other hand, in 4-azidopyrimidine analogues, the azido group is at the ortho and para positions with respect to the N1 and N3 ring nitrogens. Based on our ESR studies and theoretical results of HFCC as well as spin density distribution calculations, the azido group at $\mathrm{C} 4$ in the pyrimidine base makes the azide anion radical more 
delocalized and hence more stable in comparison to other azido-modified nucleosides previously studied ${ }^{104-106}$ and to the azido group in 5AzU.

\subsubsection{From azido-modified purine nucleosides (2-N3-dGuo)}

We also irradiated 2-azido-2'-deoxyinosine $(\mathbf{8 9}, 2$-Az-dGuo) to test for formation of the elusive guanyl aminyl radical $\left(\mathrm{G}(\mathrm{N} 2-\mathrm{H}) \bullet \cdot{ }^{90-93,95,219}\right.$ The ESR spectrum obtained from 2-Az-dGuo sample is compared with the ESR spectrum found from a matched sample of the tetrazole compound and to the authentic spectrum of $\mathrm{G}(\mathrm{N} 2-\mathrm{H}) \bullet$ that was obtained via one-electron oxidation of 1-methyl-dGuo and in DNA-oligomers. ${ }^{92,93,219}$

The spectra in panel A of Figure 58 shows that the ESR spectrum obtained from radiation-produced electron addition at $77 \mathrm{~K}$ to $2-\mathrm{Az}-\mathrm{dGuo} \mathbf{8 9}$ followed by annealing to $135 \mathrm{~K}$ for $15 \mathrm{~min}$ is very similar to the corresponding spectra found from tetrazole $\mathbf{1 1 3}$. This result concludes that addition of radiation-produced electron to 2-Az-dGuo 89 and to tetrazole 113 (analogous to protected 4-azido-2'-deoxyuridine 112) led to $\mathrm{RN}_{3}{ }^{- \text {- }}$ formation. The HFCC values of the two axially symmetric anisotropic $\mathrm{N}$ of $\mathrm{RN}_{3}{ }^{-}$from $\mathbf{8 9}$ are very close to those for $\mathrm{RN}_{3}{ }^{\bullet-}$ from 113 .

Comparison of the authentic spectrum of $\mathrm{G}(\mathrm{N} 2-\mathrm{H}) \bullet$ that was obtained via one-electron oxidation of 1-methyl-dGuo (black spectrum in panel B, Figure 58) with the ESR spectrum obtained from radiation-produced electron addition to 2-Az-dGuo 89 (panel A) clearly shows that the two spectra are not identical in terms of lineshape, overall hyperfine splitting, and the center of the spectra. Thus, electron addition to 2-Az-dGuo does not lead to the formation of $\mathrm{G}(\mathrm{N} 2-\mathrm{H}) \bullet$ unlike the reaction of radiation-produced electron with

azides that are directly linked to the sugar moiety ${ }^{104,105}$ or with azides at C5 of the pyrimidine ring, ${ }^{106}$ including 5-AzU. 

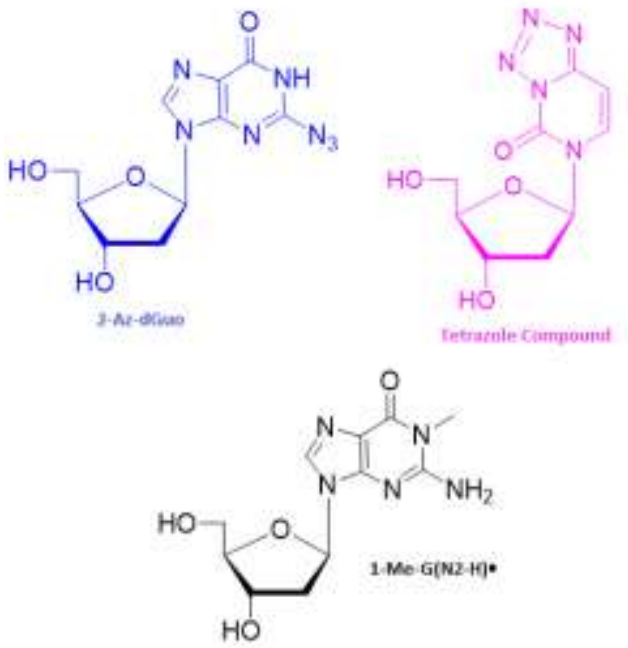

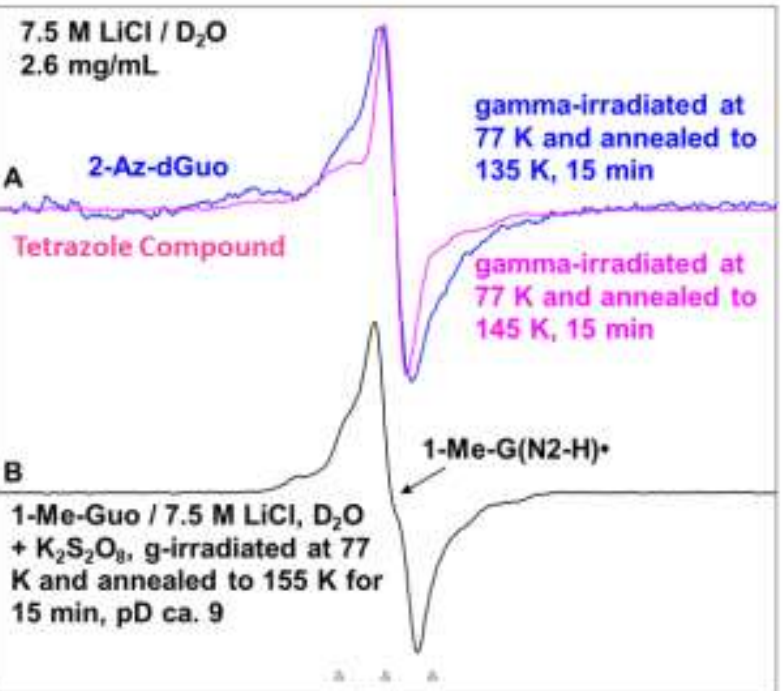

Panel (A) ESR spectrum obtained from radiation-produced (absorbed dose of $\gamma$-radiation $=600 \mathrm{~Gy}$ in the dark) electron addition at $77 \mathrm{~K}$ to matched samples of $\mathbf{8 9}$ (2-Az-dGuo;2-3 $\mathrm{mg} / \mathrm{mL}$, blue) followed by annealing to $145 \mathrm{~K}$ for $15 \mathrm{~min}$ in the dark and of tetrazole 113 (2-3 $\mathrm{mg} / \mathrm{mL}$, pink) followed by annealing to $135 \mathrm{~K}$ for $15 \mathrm{~min}$ in the dark. The line components due to $\mathrm{Cl}_{2}{ }^{-}$are subtracted from these recorded spectra. Panel (B) authentic ESR spectrum of the $\mathrm{G}(\mathrm{N} 2-\mathrm{H}) \bullet$ from 1-methyl-dGuo (1-Me- $\mathrm{G}(\mathrm{N} 2-\mathrm{H}) \bullet$ ) ( $\gamma$-irradiation absorbed dose at $77 \mathrm{~K}=2.5 \mathrm{kGy}$ ) via one-electron oxidation by $\mathrm{Cl}_{2}{ }^{-}$due to annealing at ca. $155 \mathrm{~K}$ for $15 \mathrm{~min}$ in the dark at $\mathrm{pD}$ ca. 9 in homogeneous glass $\left(7.5 \mathrm{M} \mathrm{LiCl}\right.$ in $\left.\mathrm{D}_{2} \mathrm{O}\right)$ in the presence of the electron scavenger $\mathrm{K}_{2} \mathrm{~S}_{2} \mathrm{O}_{8}(6-8 \mathrm{mg} / \mathrm{mL})$.

Figure 58. Comparison of EPR spectra acquired for 2-azido-2'-deoxyinosine 89, 4tetrazolo-2'-deoxyuridine 113, and 2'-deoxy- $N^{1}$-methylguanosine.

In 2-Az-dGuo, the azido moiety is at the ortho position with respect to either N1 or N3 of the guanine ring, similar to how the azide group is ortho to N3 in $\mathbf{1 1 2}$. Therefore, formation of the azide anion radical, as found in the tetrazole $\mathbf{1 1 3}$ and in 4-azido 112, is expected also from 2-Az-dGuo samples rather than the neutral aminyl radical and is indeed observed. 


\subsection{Photoexcitation of $\mathrm{RN}_{3}{ }^{*}$ from $2-\mathrm{Az}-\mathrm{dGuo}$ leads to the formation of}

\section{sugar radicals, possibly $\mathrm{C5}^{\prime} \bullet$}

Similar to our findings of sugar radical formation via photoexcitation of $\mathrm{RN}_{3}{ }^{\circ}$ from tetrazole $\mathbf{1 1 3}$ and in 4-azido 112 (Figure 56), and to the work of Adhikary-Sevilla laboratory showing that excited states of purine and pyrimidine cation radicals can lead to formation of sugar radicals ${ }^{93-95,219,227,229}$, photoexcitation of a separately prepared and matched 2-Az-dGuo sample was carried out employing a photoflood lamp at $77 \mathrm{~K}$. The results are presented in Figure 59 below. 


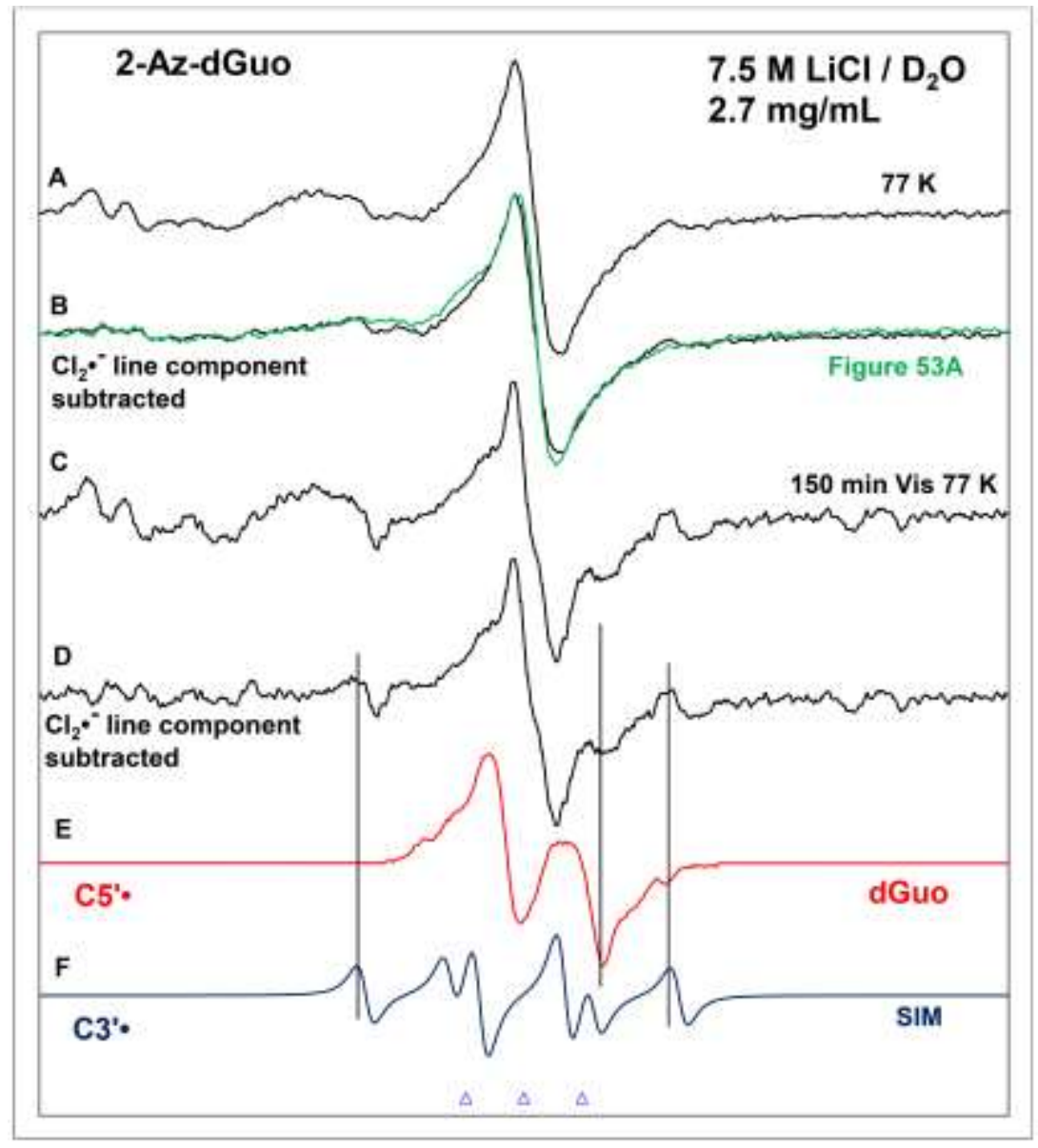

(A) Spectrum (black) is obtained at $77 \mathrm{~K}$ after $\gamma$-irradiation (absorbed dose $=600 \mathrm{~Gy}$ ) at $77 \mathrm{~K}$ in the dark. (B) Spectrum (black) after subtraction of line components of $\mathrm{Cl}_{2}{ }^{-}$from spectrum (A). The green spectrum is the spectrum of $\mathrm{RN}_{3}{ }^{-}$from 2-Az-dGuo in Figure 53A which is superimposed on the black spectrum for comparison. (C) Spectrum (black) recorded at $77 \mathrm{~K}$ after photoexcitation at $77 \mathrm{~K}$ for $150 \mathrm{~min}$. (D) Spectrum (black) after subtraction of line components of $\mathrm{Cl}_{2}{ }^{-}$from spectrum $(\mathrm{C})$. (E) The red spectrum is that of $\mathrm{C}^{\prime} \bullet$, produced from $\mathrm{G}^{\bullet^{+}}$in 8 -D-3'-dGMP via photoexcitation. ${ }^{227}$ (F) The simulated spectrum of $\mathrm{C}^{\prime} \cdot$ (for details of simulation parameters, see text). The line components of C3'• and C5'• in spectrum (D) are indicated by solid lines.

Figure 59. ESR spectra obtained from a 2-Az-dGuo 89 after radiation-produced electron addition and UV photoexcitation

The black spectrum in panel A was immediately recorded after $\gamma$-radiation at $77 \mathrm{~K}$ of the homogeneous glassy sample of 2-Az-dGuo $89\left(2-3 \mathrm{mg} / \mathrm{ml}\right.$ in $7.5 \mathrm{M} \mathrm{LiCl} / \mathrm{D}_{2} \mathrm{O}(\mathrm{pH} / \mathrm{pD}$ ca. 5)) The black spectrum in panel (B) was obtained after subtraction of line components 
of $\mathrm{Cl}_{2}{ }^{-}$from spectrum (A) following procedures published in previous work. ${ }^{94}$ For comparison, the spectrum (green) of $\mathrm{RN}_{3} \bullet^{-}$from 2-Az-dGuo (blue spectrum in Figure 59A) is superimposed on the black spectrum and it is assigned to $\mathrm{RN}_{3} \bullet^{-}$. Subsequently, the glassy sample was subjected to visible photoexcitation at $77 \mathrm{~K}$ employing a photoflood lamp for 150 min and the ESR spectrum (black) is shown in panel (C). Analogous to spectrum (B), spectrum (D) was obtained after subtraction of line components of $\mathrm{Cl}_{2} \bullet$ - from spectrum (C). Comparison of spectra (C) and (D) obtained after photoexcitation with spectra (A) and (B) obtained before photoexcitation, clearly show development of new line components. The red spectrum (E) of $\mathrm{C}^{\prime} \cdot\left(\right.$ an anisotropic doublet due to alpha proton at $\mathrm{C}^{\prime}$ ' of ca. 19 $\mathrm{G}$ ), produced from $\mathrm{G}^{\bullet^{+}}$in $8-\mathrm{D}-3^{\prime}-\mathrm{dGMP}$ via photoexcitation, ${ }^{227}$ and the simulated spectrum of $\mathrm{C}^{\prime} \cdot[$ blue spectrum $(\mathrm{F})]$ were used to compare against our obtained spectrum (D). Using these as standards, our results shows ca. $40 \%$ conversion of $\mathrm{RN}_{3} \bullet^{-}$from 2 -AzdGuo 89 to sugar radicals $\left(\mathrm{C}^{\prime} \bullet(\right.$ ca. $30 \%)$ and $\mathrm{C}^{\prime} \bullet($ ca. 10\%)) upon photoexcitation. The line components of $\mathrm{C}^{\prime} \bullet$ and $\mathrm{C}^{\prime} \bullet$ in spectrum (D) are indicated by solid lines. These results show the low reactivity of $\mathrm{RN}_{3} \bullet^{-}$from purine nucleosides, similar to our findings on low reactivity of $\mathrm{RN}_{3} \bullet^{-}$from pyrimidine nucleosides.

The blue spectrum in panel $\mathrm{F}$ is simulated employing 1 isotropic beta H HFCC (19.2 $\mathrm{G}$, at $\mathrm{C}^{\prime}$ ') and 2 isotropic beta $\mathrm{H} \mathrm{HFCC} \mathrm{(25.7} \mathrm{G} \mathrm{each,} \mathrm{at} \mathrm{C2')} \mathrm{with} 4.0 \mathrm{G}$ isotropic linewidth and $g=2.0031$. The outer lines of the simulated C $3^{\prime} \cdot$ spectrum match those in spectrum (D) as indicated by solid lines. Depending upon the conformation of the beta hydrogens with respect to the radical site p-orbital at $\mathrm{C}^{\prime}$ in $\mathrm{C}^{\prime} \bullet$, the $\mathrm{HFCC}$ values of beta $\mathrm{H}$ would vary and hence a number of $\mathrm{C}^{\prime} \bullet$ spectra with different beta H HFCC values have been reported in the literature..$^{219,227,229,230}$ 
The mechanism of sugar radical formation from excited $\mathrm{RN}_{3} \bullet^{-}$from 2 -Az-dGuo 89 (Scheme 50) is similar to that in pyrimidines (scheme 49). Predominant formation of C3'• via photoexcitation of $\mathrm{RN}_{3}{ }^{-}$from 89 is found to be similar to significant formation of C3'• via photoexcitation of guanine cation radical from dGuo and from 5'-dGMP at low temperatures. $^{227}$

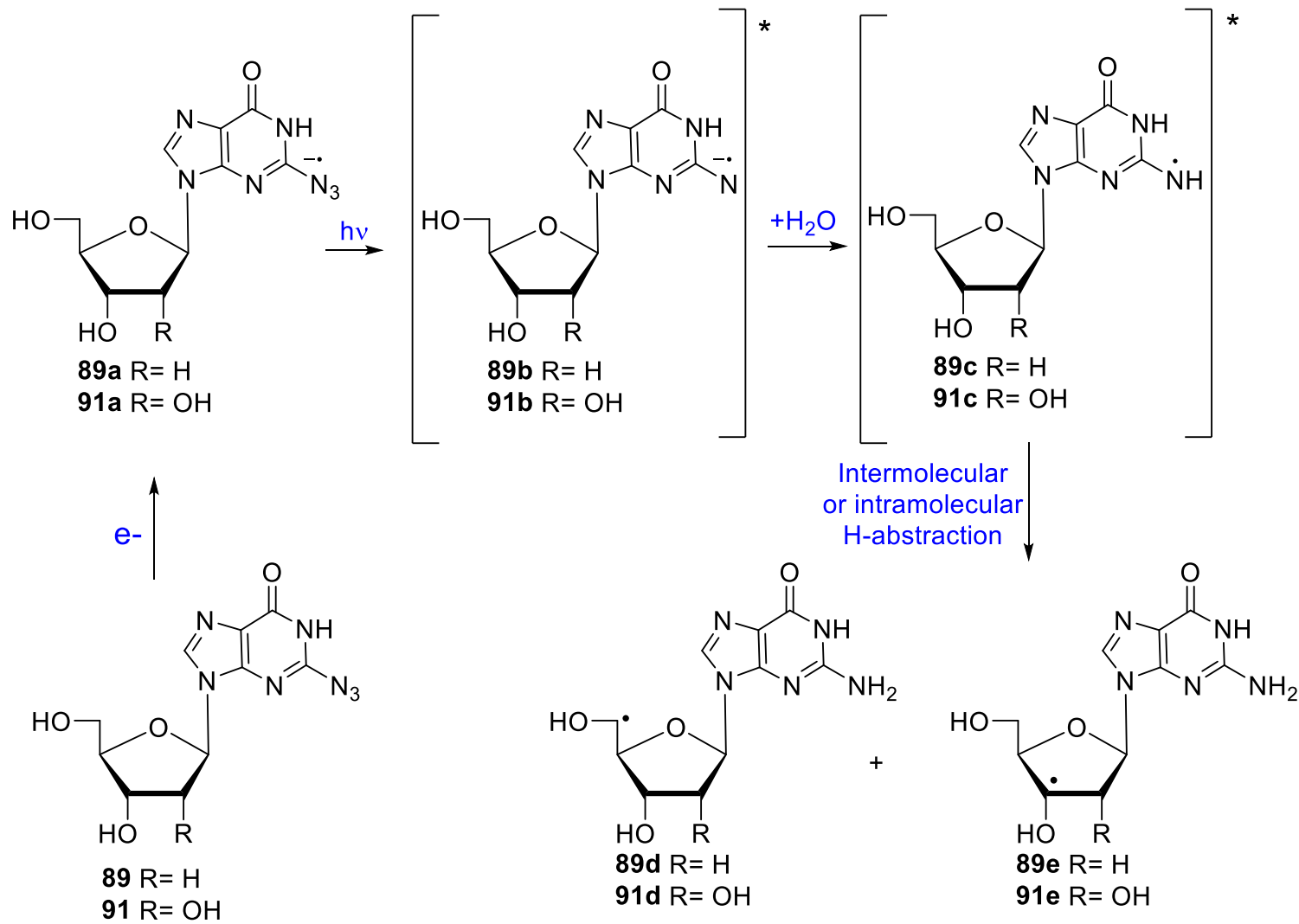

Scheme 50. Proposed mechanism for the formation of C5' and C3' centered carbon radicals upon UV-photoexcitation of the RN3•- in 2-azidoguanosine derivatives.

In conclusion, ESR studies of electron addition to azido- or tetrazole-modified nucleosides clearly establish that position of the azido group in the base moiety dictates its reactivity towards electron addition. If the azido group is "isolated" (e.g., in the "meta position" to pyrimidine nitrogen's as in 5-AzU, in the sugar moiety as in $3^{\prime}-\mathrm{AZT}$, or indirectly attached to the aromatic ring as in 5-AmdU or 5-AvdU), then it is rapidly 
converted to neutral aminyl radical and is expected to cause radiosensitization based on the studies of AmdU in EMT6 cancer cells. ${ }^{106}$ However, if the azido group is in ortho/para position to ring nitrogens as in 4-azido $\mathbf{1 1 2}$, or tetrazole $\mathbf{1 1 3}$ as well as in 6-azidopurine $\mathbf{X X}$, these compounds produce $\mathrm{RN}_{3}{ }^{{ }^{-}}$which is stable and does not rapidly convert to the aminyl radical except in their excited state. Hence, these compounds are not expected to show much radiasensitization in comparison to the significant radiosensitization observed from 5-AmdU. Of course, 5-AzU is predicted to show significant radiosensitization as it produces aminyl radical immediately after irradiation at $77 \mathrm{~K} .{ }^{85}$

\subsubsection{Biological evaluation of azido-modified nucleosides in vivo}

Given our discovery of the nitrogen and sugar carbon centered radicals, we tested the radiosensitizing potential of these azido compound in vivo. Each class of azido-modified nucleosides were tested in Dr. Pathak's laboratory in University of Arkansas for Medical Sciences. These included 2-azido-2'-deoxyinosine 89, 6-azido-2'-deoxypurine nucleoside 107, 4-azido-3',5'-di- $O$-acetyl-2'-deoxyuridine 112, and 4-tetrazolo-2'-deoxyuridine 113.

The azido analogues were tested in MDA-MB-231 (epithelial breast cancer cell line), MCF7 (breast cancer cell line) and U87 (glioblastoma cell line) cells. MDA-MB-231, MCF7 and U87 cells were seeded in 96 well plate (10,000 cells/well). Cells were incubated with $100 \mu \mathrm{M}$ of compound. After $24 \mathrm{~h}$ of treatments, cells were irradiated with $2 \mathrm{~Gy}$ (Grey) and $4 \mathrm{~Gy}$ of gamma radiation. After $24 \mathrm{~h}$ and $48 \mathrm{~h}$ of irradiations cell viability was checked with MTS cell proliferation assay (Promega). The results in Figures 60-62 show that all the compounds tested showed only minor radiosensitizing effects in any of the cancer cell lines tested. These results are supported by the fact that $\mathrm{C} 5$ ' centered radicals have been 
known to be quite stable and form oxidation products which do not results in DNA strand breakage. $^{231}$
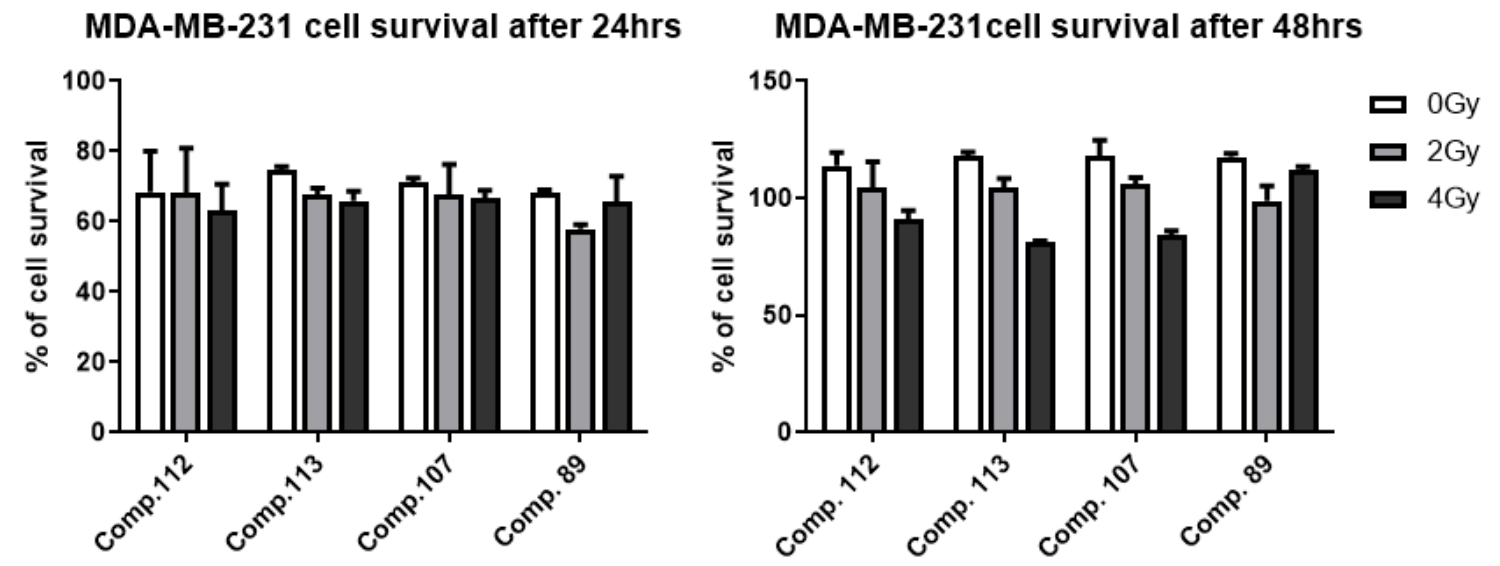

Figure 60. Survival data of MDA-MB-231 cell lines after incubation with azido modified nucleosides and gamma radiation. Data is shown for survival rates after 24 and 48 hours.
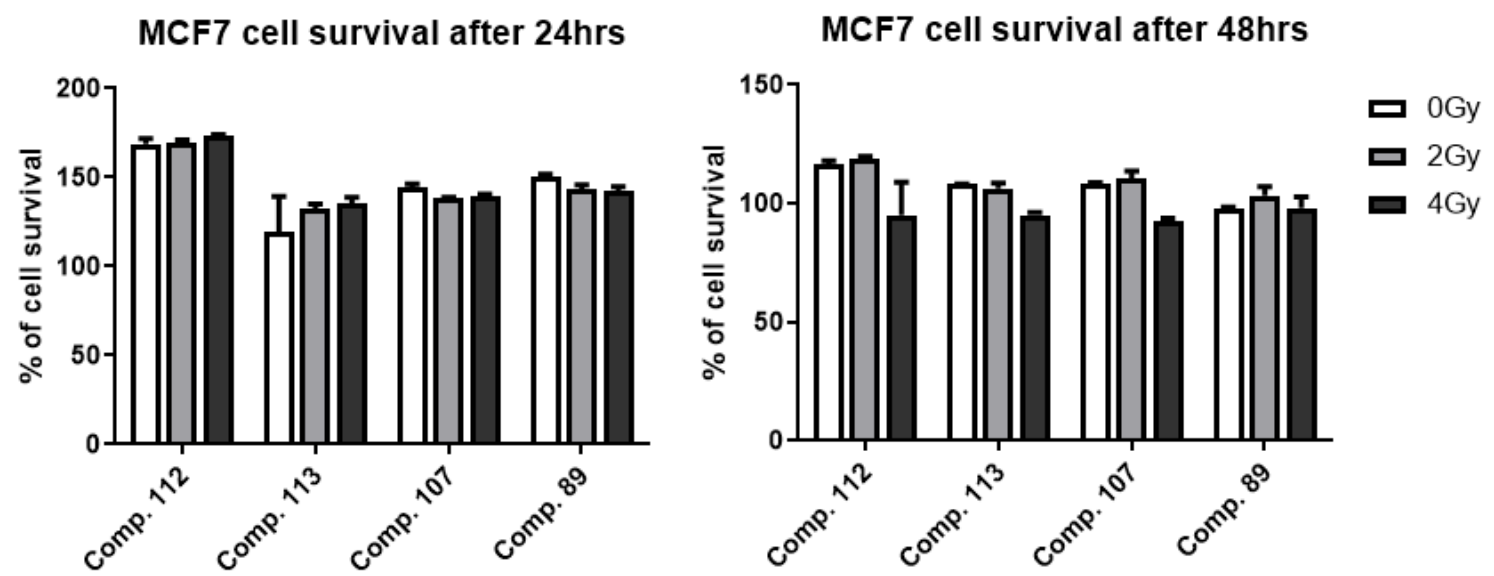

Figure 61. Survival data of MCF7 cell lines after incubation with azido modified nucleosides and gamma radiation. Data is shown for survival rates after 24 and 48 hours. 

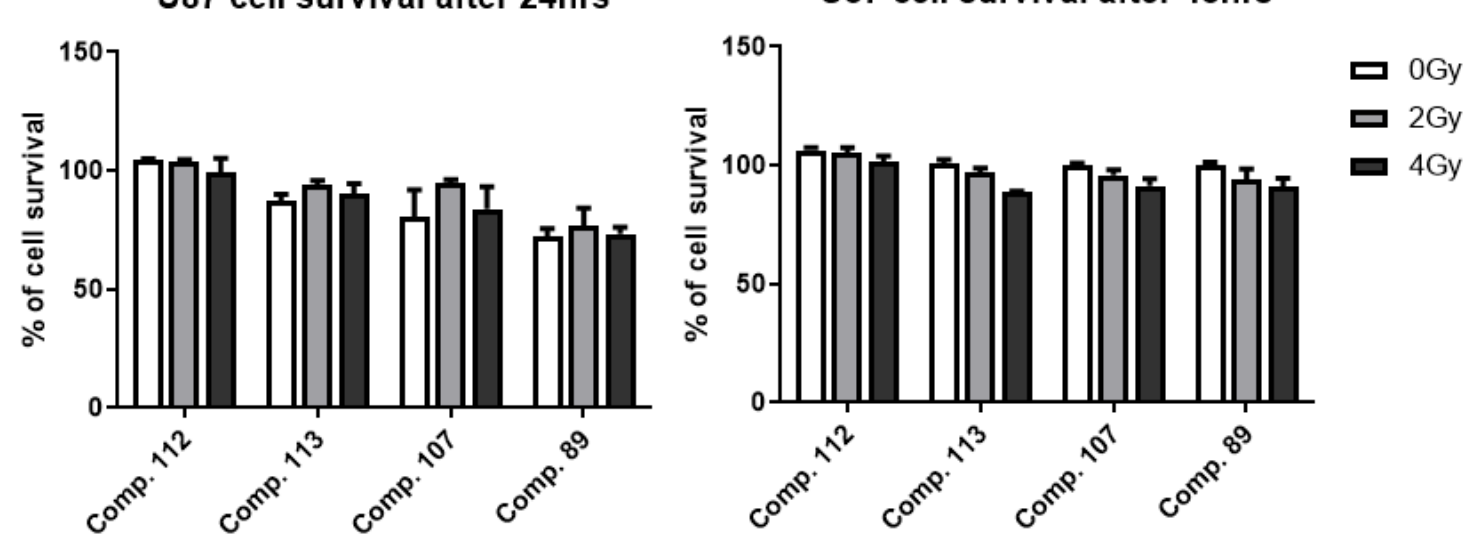

Figure 62. Survival data of U87 cell lines after incubation with azido modified nucleosides and gamma radiation. Data is shown for survival rates after 24 and 48 hours.

\subsubsection{Synthesis of 4-azido-2'-deoxy-5',5'-dideuteropyrimidine nucleoside to study formation of $\mathrm{C5}^{\prime}$ centered radical}

Given our preliminary results showing formation of a C5' centered radical in EPR studies of the azido-modified nucleosides, we made an effort to synthesize 5',5"-dideutero analogues of the 4-azidopyrimidine $\mathbf{1 1 2}$ and 4-tetrazolopyrimidine $\mathbf{1 1 3}$ nucleosides to verify our findings through EPR. We expected that the anisotropic doublet seen from the coupling of the carbon centered radical to C5'-hydrogen would be eliminated in EPR studies of 5',5"-dideutero analogues. Retrosynthetic analysis shows that synthesis of $\mathbf{1 1 9}$ and $\mathbf{1 2 0}$ could be undertaken by coupling 1-O-acetate-3,5-di- $O$-acetyl-5,5'-dideutero-2deoxyribofuranose $\mathbf{1 2 3}$ with persilylated uracil base $\mathbf{1 2 2}$ (Figure 63). Further elaboration of the 4-position hydroxyl group of uracil ring in 3',5'-di-O-acetyl-2'-deoxy-5',5'dideuterouridine $\mathbf{1 2 1}$ should afford desired azido analogues in similar yield as described above. 


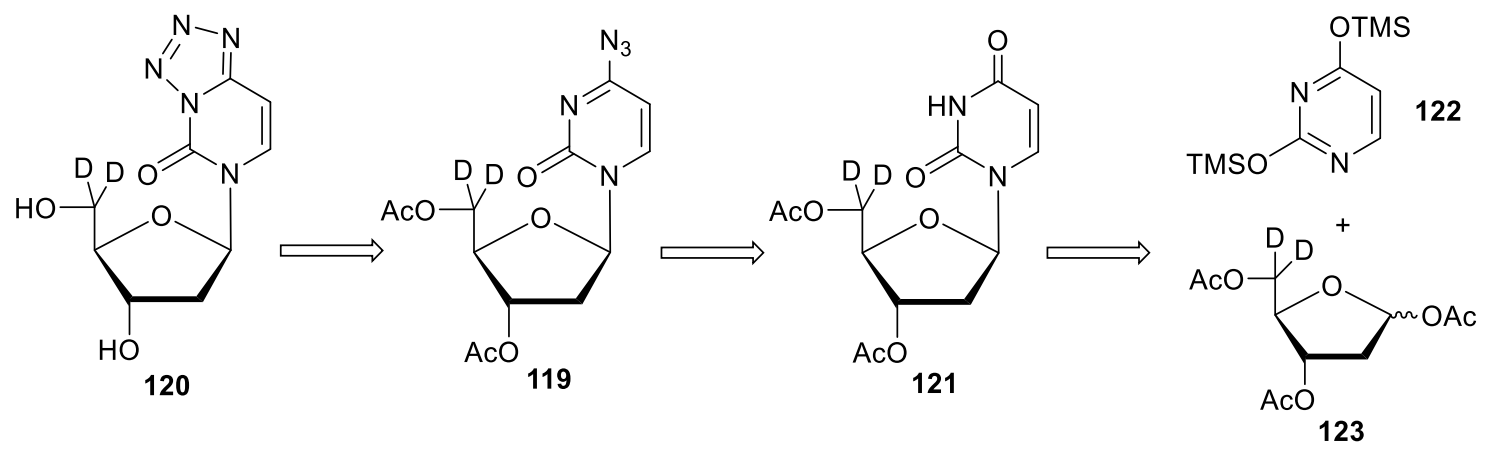

Figure 63. Retrosynthetic analysis for the synthesis of 4-azido-2'-deoxy-5',5"dideuteropyrimidine nucleoside

\subsubsection{Synthesis of 3',5'-di-O-acetyl-2'-deoxyuridine for model studies}

In order to develop an effective protocol for synthesis of 4-azido-2'-deoxy-5',5"dideuteropyrimidine nucleoside (e.g. 119 and 120), we first attempted synthesis of undeuterated $3^{\prime}, 5^{\prime}$-di- $O$-acetyl-2'-deoxyuridine $\mathbf{1 1 0}$ by coupling of $1-O$-acetate-3,5-di- $O$ acetyl-2'-deoxyribofuranosyl $\mathbf{1 2 8}$ with persilylated uracil 122. Synthesis of 1,3,5-tri- $O$ acetyl-2'-deoxyribose was undertaken through multistep protocols previously described in literature. $^{232-235}$ Thus, 2-deoxyribose $\mathbf{1 2 5}$ was methylated using catalytic amounts of concentrated $\mathrm{H}_{2} \mathrm{SO}_{4}$ in methanol to give $\mathbf{1 2 6}$ (85\% as a mixture of $\alpha / \beta$ anomers of deoxyribofuranose and deoxyribopyranose derivatives; Scheme 51). Treatment of the mixture of methyl 2-deoxyribofuranosides $\mathbf{1 2 6}$ with acetic anhydride and pyridine resulted in acetylation of the 3 and 5 positions to give $\mathbf{1 2 7}$ in $61 \%$ yield. Acetolysis with sulfuric acid in acetic anhydride/acetic acid yielded 1-O-acetate-3,5-di- $O$-acetyl-2'deoxyribofuranose 128 (79\%). All sugar products were obtained in a mixture of $\alpha / \beta$ anomers of deoxyribofuranose and deoxyribopyranose products, which were not separated for the subsequent steps. 


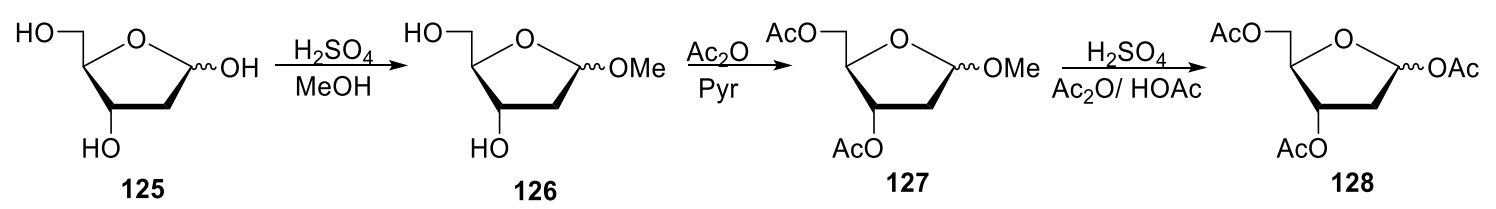

(for simplicity only deoxyribofuranose isomers are shown)

Scheme 51. Synthesis of 1,3,5-tri- $O$-acetyl-2'-deoxyribose for coupling reaction

Persilylation of uracil $\mathbf{1 2 9}$ with chlorotrimethylsilane and hexamethyldisilazane gave 122 (Scheme 52). Yield was expected to be quantitative based on literature. ${ }^{105}$ Coupling of sugar 128 and persilylated uracil $\mathbf{1 2 2}$, in $\mathrm{CH}_{3} \mathrm{CN}$, in the presence of trimethylsilyl trifluoromethanesulfonate (TMSOTf) catalyst afforded $82 \%$ overall conversion to the mixture of protected uridine analogues. This mixture contained desired $\beta$-uridine product 110 (as confirmed with NMR standard) as well as $\alpha$-uridine anomer, along with $\alpha / \beta$ deoxyribopyranosyl nucleoside byproducts. Purification by silica gel column chromatography afforded 3',5'-di-O-acetyl-2'-deoxyuridine $\mathbf{1 1 0}(20 \%$ as analyzed by NMR) in mixture with its $\beta$-deoxyribopyranosyl derivative 110a

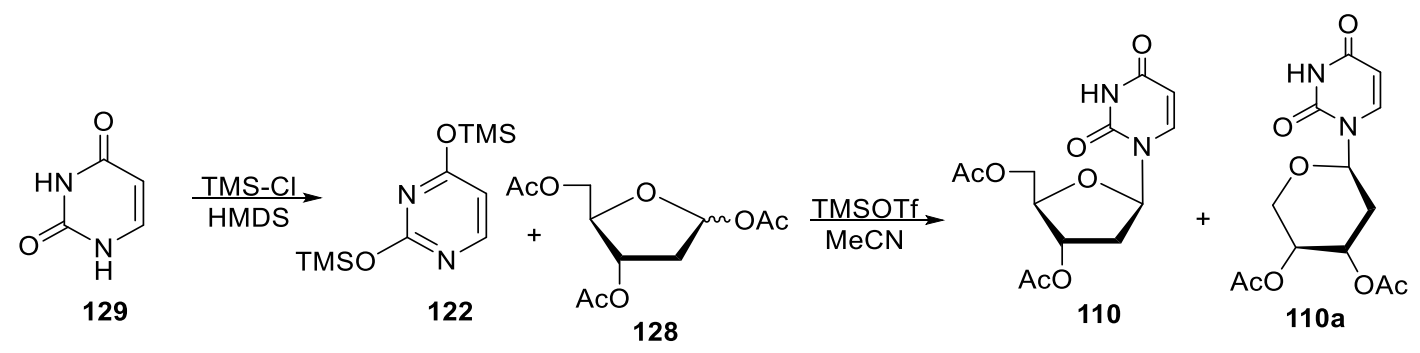

Scheme 52. Synthesis of 3',5'-di- $O$-acetyl-2'-deoxyuridine

\subsubsection{Synthesis of 3',5'-di- $O$-acetyl-2'-deoxy 5',5'-dideuterouridine and its 4-} azido and 4-tetrazolo derivatives

Once we developed an adequate protocol for the synthesis of 3',5'-di- $O$-acetyl-2'deoxyuridine 110, we set out synthesize 3',5'-di-O-acetyl-2'-deoxy-5',5"-dideuterouridine 121 through analogous methods. We began by methylation of commercially available 2 deoxy-D-5,5'-dideuteroribose $\mathbf{1 3 0}$ using $\mathrm{H}_{2} \mathrm{SO}_{4}$ in methanol to give $\mathbf{1 3 1}$ in $94 \%$ yield 
(Scheme 53). Subsequent acetylation of the 3 and 5 position using $\mathrm{Ac}_{2} \mathrm{O} /$ pyridine yielded $80 \%$ of 132. Finally, acetolysis of $\mathbf{1 3 2}$ using $\mathrm{H}_{2} \mathrm{SO}_{4}$ in a mixture of $\mathrm{Ac}_{2} \mathrm{O} / \mathrm{AcOH}$ (1:2) gave $82 \%$ of 1,3,5-tri- $O$-acetyl-2-deoxy-5,5'-dideuteroribose 123. All sugar products were obtained as a mixture of deoxyribofuranose and deoxyribopyranose anomers but were not separated throughout the reaction sequense. It is interesting to note that unlike in the synthesis of 128, where each step produced four isomers in roughly equal yield $(\alpha / \beta$ deoxyribofuranose and $\alpha / \beta$ deoxyribopyranose), synthesis of $\mathbf{1 2 3}$ produced only two major products which accounted for $>90 \%$ of yield. These products were attributed to $\beta$ deoxyribofuranose 123 and $\beta$-deoxyribopyranose.

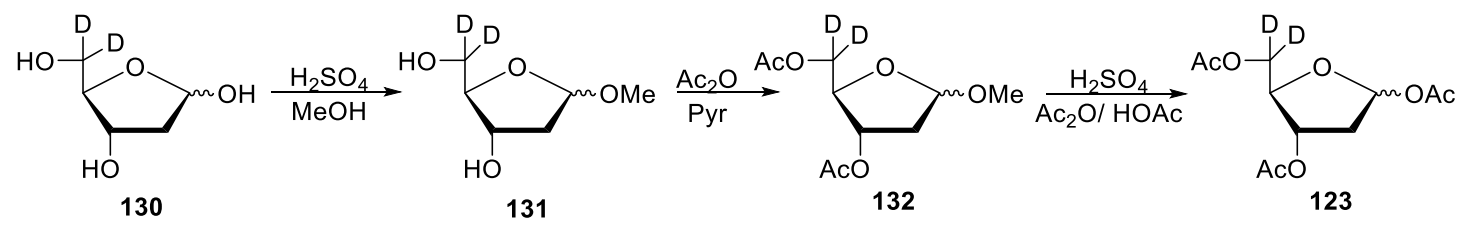

(for simplicity only deoxyribofuranose isomers are shown)

Scheme 53. Synthesis of 1,3,5-tri-O-acetyl-2-deoxy-5,5'-dideuteroribose for subsequent coupling reaction

Coupling of sugar $\mathbf{1 2 3}$ with persilylated uracil $\mathbf{1 2 2}$ in the presence of TMSOTf in $\mathrm{CH}_{3} \mathrm{CN}$ gave roughly $50 \%$ of desired 2'-deoxyuridine 121 (Scheme 54) along with deoxyribopyranosyl derivative 121a. Yield of $\mathbf{1 2 1}$ was calculated based on ${ }^{1} \mathrm{H}$ NMR analysis. The mixture of $\mathbf{1 2 1}$ with other isomers was not separable through column chromatography and, thus, was used for the subsequent reactions. 


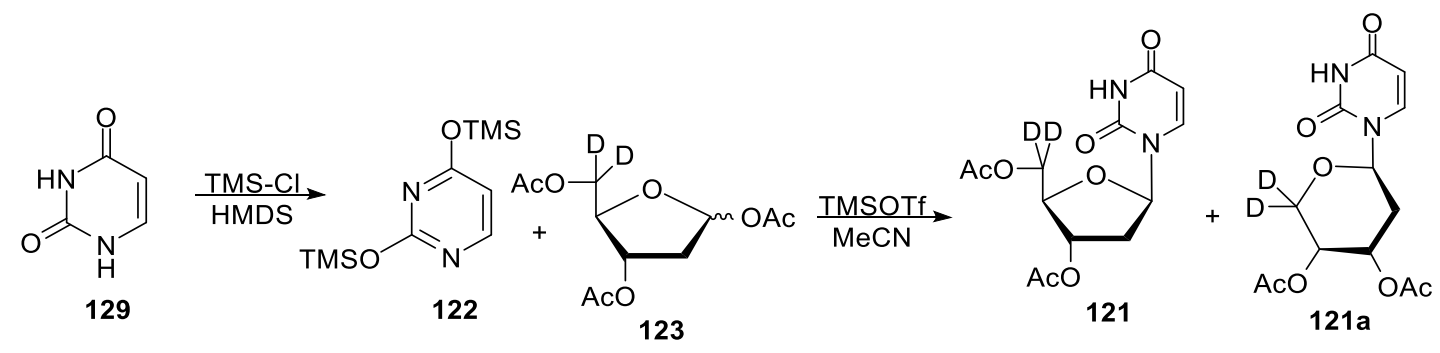

Scheme 54. Synthesis of 3',5'-di- $O$-acetyl-2'-deoxy-5',5"-dideuterouridine

Treatment of 121 with $\mathrm{POCl}_{3}, \mathrm{Et}_{3} \mathrm{~N}$, and 1- $\mathrm{H}$-1,2,4-triazole in $\mathrm{CH}_{3} \mathrm{CN}$ overnight gave roughly $25 \%$ of triazole 133 along with its deoxyribopyranosyl analogue. ${ }^{151}$ Then, $\mathbf{1 3 3}$ was reacted with $\mathrm{NaN}_{3}$ and $\mathrm{CH}_{3} \mathrm{COOH}$ in a mixture of $\mathrm{DMF} / \mathrm{H}_{2} \mathrm{O}$ for several days to yield azide $134(45 \%)$. HRMS confirmed the molecular formula and mass of compound $\mathbf{1 3 4}$ and its ${ }^{1} \mathrm{H}$ NMR was identical to 112 except for the disappearance of peaks for H5',5" and simplification of H4' splitting. Azide 134 was sent for EPR studies to confirm C5' radical formation. Depending on the EPR results from azide 134, the latter is envisioned to be converted to tetrazole 135 by reaction with saturated $\mathrm{HCl}$ in $\mathrm{MeOH}$, analogously to protocol described for the synthesis of 113. Attempts will be also made to obtain pure tetrazole 135 from other isomers by reverse phase HPLC for further EPR studies.

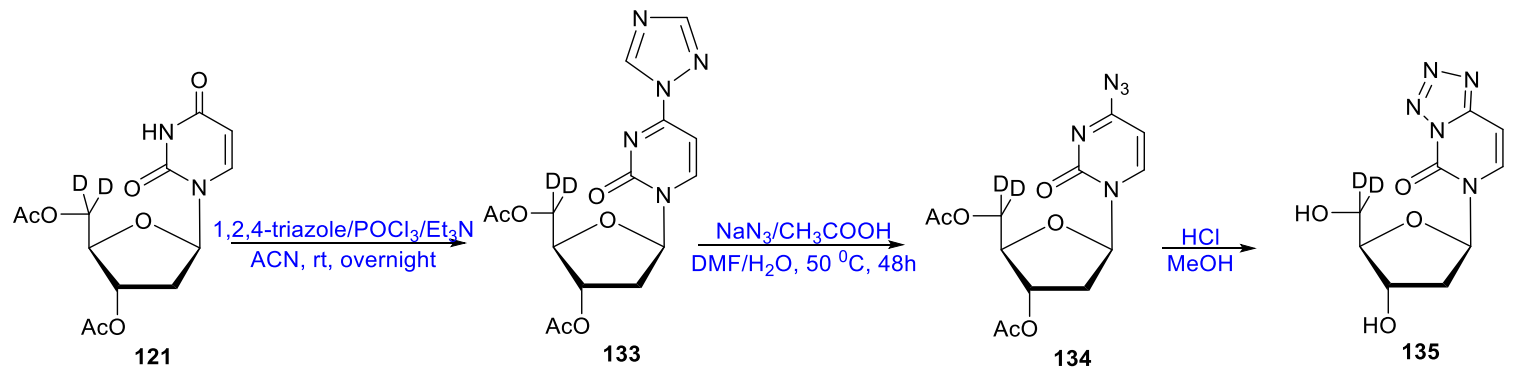

(for simplicity only deoxyribofuranosyl products are shown)

Scheme 55. Synthesis of 3',5'-di-O-acetyl-4-azido-5',5''-dideutero-2'-deoxyuridine 


\section{EXPERIMENTAL PROCEDURES}

\subsection{General procedures}

The ${ }^{1} \mathrm{H}(400 \mathrm{MHz}),{ }^{13} \mathrm{C}(100 \mathrm{MHz})$, or ${ }^{19} \mathrm{~F}(376 \mathrm{MHz})$ NMR spectra were recorded at ambient temperature in deuterated solvents, as specified. Reactions were carried out under $\mathrm{N}_{2}$ atmosphere unless otherwise indicated. The reactions involving non-radioactive materials were followed by TLC with Merck Kieselgel $60-\mathrm{F}_{254}$ sheets and products were detected with a $254 \mathrm{~nm}$ UV lamps. Column chromatography was performed using Merck Kieselgel 60 (230-400 mesh). Perkin Elmer high-performance liquid chromatography (HPLC) was used for purification on RP-C18 semi-preparative Phenomenex Gemini or preparative XTerra columns with mobile phase and flow rate as indicated. High-resolution mass spectra (HRMS) were obtained with a Solarix instrument (FT-MS). The SCN-BnNOTA and NODA-GA(tBu $)_{3}$ bifunctional chelators were purchased from Macrocyclics Inc, TX, USA and CheMatech, Dijon, France, respectively. Gemcitabine hydrochloride was purchased from LC Laboratories, MA, USA. All other reagent grade chemicals were purchased from Sigma Aldrich. Data in section 4.2 has been reprinted with permission from the publisher ${ }^{236}$ and authors. ${ }^{206,207}$

\subsection{Synthesis and evaluation of 4-N-Alkyl gemcitabine analogues}

\subsubsection{Synthesis of 4- $N$-alkylgemcitabine with terminal fluoromethyl ketone}

4- $N$-(p-Toluenosulfonyl)-2'-deoxy-2',2'-difluorocytidine (58). Gemcitabine 57 (600 $\mathrm{mg}, 2.0 \mathrm{mmol}$ ) was dried for several hours over $\mathrm{P}_{2} \mathrm{O}_{5}$, then dissolved in $10 \mathrm{~mL}$ of anhydrous pyridine in a flame dried flask. TMS-Cl $(5.1 \mathrm{~mL})$ was added to the suspension. After $2 \mathrm{~h}$, tosyl chloride ( $\mathrm{TsCl}, 3.8 \mathrm{~g}, 20 \mathrm{mmol}$ ) was added, and the reaction was gradually heated to 
$60^{\circ} \mathrm{C}$ with stirring. After $20 \mathrm{~h}$, the volatiles were evaporated, and the resulting mixture was treated with $\mathrm{MeOH} / \mathrm{NH}_{3}(10 \mathrm{~mL})$ and stirred at room temperature for $24 \mathrm{~h}$. The volatiles were then evaporated and the mixture was column chromatographed (90\% EtOAc/Hex) to give $\mathbf{5 8}^{33}(714 \mathrm{mg}, 85 \%)$ as a white solid: ${ }^{1} \mathrm{H} \mathrm{NMR}\left(\mathrm{CD}_{3} \mathrm{OD}\right) \delta 2.42\left(\mathrm{~s}, 3 \mathrm{H}, \mathrm{CH}_{3}\right), 3.78(\mathrm{dd}$, $\left.J=3.4,12.8 \mathrm{~Hz}, 1 \mathrm{H}, \mathrm{H} 5^{\prime}\right), 3.90-3.95$ (m, 2H, H4,H5"), 4.28 (dt, $J=8.4,12.0 \mathrm{~Hz}, 1 \mathrm{H}$, H3'), $6.13\left(\mathrm{dd}, J=5.3,9.5 \mathrm{~Hz}, 1 \mathrm{H}, \mathrm{H} 1^{\prime}\right), 6.65$ (d, $\left.J=8.2 \mathrm{~Hz}, 1 \mathrm{H}, \mathrm{H} 5\right), 7.36(\mathrm{~d}, J=8.0 \mathrm{~Hz}$, 2H, Ar), $7.79(\mathrm{~d}, J=8.3 \mathrm{~Hz}, 2 \mathrm{H}, \mathrm{Ar}), 7.99(\mathrm{~d}, J=8.1 \mathrm{~Hz}, 1 \mathrm{H}, \mathrm{H6}) ;{ }^{13} \mathrm{C}$ NMR $\delta 21.43$, 60.34 (C5'), 70.21 (dd, $\left.J=18.8,27.2 \mathrm{~Hz}, \mathrm{C}^{\prime}\right), 82.99$ (d, $\left.J=8.4, \mathrm{C} 4^{\prime}\right), 85.46$ (dd, $J=23.9$, $\left.41.3 \mathrm{~Hz}, \mathrm{C} 1^{\prime}\right), 98.46$ (C5), 123.84 (t, $J=258.7 \mathrm{~Hz}, \mathrm{C} 2$ '), 127.58 (Ar), 130.52 (Ar), 140.71 (Ar), 142.62 (C6), 144.66 (Ar), 150.21 (C2), $160.54(\mathrm{C} 4) ;{ }^{19} \mathrm{~F}$ NMR $\delta-120.17$ (br d, 1F), -119.41 (dd, $J=4.1,12.7 \mathrm{~Hz}, 1 \mathrm{~F})$.

4- $N$-(Undec-10-en-1-yl)-2'-deoxy-2',2'-difluorocytidine (59). To a stirred solution of $58(200 \mathrm{mg}, 0.48 \mathrm{mmol})$ in 1,4-dioxane $(5 \mathrm{~mL})$ and $\mathrm{Et}_{3} \mathrm{~N}(134 \mu \mathrm{L}, 97.2 \mathrm{mg}, 0.96 \mathrm{mmol})$ was added 10-undecene amine ( $301 \mu \mathrm{L}, 243.8 \mathrm{mg}, 1.44 \mathrm{mmol})$. The mixture was stirred at $65^{\circ} \mathrm{C}$ for 48 hours. Then, the volatiles were evaporated under reduced pressure, and the resulting residue was column chromatographed $\left(5 \% \mathrm{MeOH} / \mathrm{CHCl}_{3}\right)$ to yield $\mathbf{5 9}^{33}(170 \mathrm{mg}$, 85\%): ${ }^{1} \mathrm{H}$ NMR $\left(\mathrm{CD}_{3} \mathrm{OD}\right) \delta 1.30-1.43\left(\mathrm{~m}, 12 \mathrm{H}, 6 \times \mathrm{CH}_{2}\right), 1.56-1.65\left(\mathrm{~m}, 2 \mathrm{H}, \mathrm{CH}_{2}\right)$, 2.03-2.09 (m, 2H, CH 2 ), $3.39\left(\mathrm{t}, J=7.1 \mathrm{~Hz}, 2 \mathrm{H}, \mathrm{CH}_{2}\right), 3.80\left(\mathrm{dd}, J=3.3,12.6 \mathrm{~Hz}, 1 \mathrm{H}, \mathrm{H} 5^{\prime}\right)$, $3.89\left(\mathrm{td}, J=2.8,8.3 \mathrm{~Hz}, 1 \mathrm{H}, \mathrm{H} 4^{\prime}\right), 3.95\left(\mathrm{~d}, J=12.6 \mathrm{~Hz}, 1 \mathrm{H}, \mathrm{H} 5^{\prime \prime}\right), 4.26(\mathrm{dt}, J=8.3,12.1$ $\left.\mathrm{Hz}, 1 \mathrm{H}, \mathrm{H}^{\prime}\right), 4.91-5.02\left(\mathrm{~m}, 2 \mathrm{H}, \mathrm{CH}_{2}\right), 5.82$ (tdd, $\left.J=6.7,10.3,17.0 \mathrm{~Hz}, 1 \mathrm{H}, \mathrm{CH}\right), 5.87$ (d, $J=7.6 \mathrm{~Hz}, 1 \mathrm{H}, \mathrm{H} 5), 6.23\left(\mathrm{t}, J=8.0 \mathrm{~Hz}, 1 \mathrm{H}, \mathrm{H1} 1^{\prime}\right), 7.74(\mathrm{~d}, J=7.6 \mathrm{~Hz}, 1 \mathrm{H}, \mathrm{H6}) ;{ }^{13} \mathrm{C} \mathrm{NMR}$ $\delta 28.01,29.98,30.12,30.19,30.42,30.51,30.63,34.88,41.75,60.56\left(\mathrm{C}^{\prime}\right), 70.67(\mathrm{dd}, J=$ 22.4, $23.8 \mathrm{~Hz}, \mathrm{C} 3$ '), $82.26\left(\mathrm{dd}, J=3.6,5.0 \mathrm{~Hz}, \mathrm{C}^{\prime}\right), 85.94\left(\mathrm{dd}, J=26.0,38.0 \mathrm{~Hz}, \mathrm{Cl}^{\prime}\right)$, 
97.33 (C5), 114.68, 124.05 (t, $J=258.4$ Hz, C2'), 140.16, 140.77 (C6), 158.30 (C2), 165.37

(C4); ${ }^{19} \mathrm{~F}$ NMR $\delta-119.89($ br d, $J=240.1 \mathrm{~Hz}, 1 \mathrm{~F}),-118.80($ br d, $J=240.1 \mathrm{~Hz}, 1 \mathrm{~F})$.

4- $N$-(10-Undecenyl)-3',5'-di-O-benzoyl-2'-deoxy-2',2'-difluorocytidine (60). BzCl

$(140 \mu \mathrm{L}, 168.68 \mathrm{mg}, 1.2 \mathrm{mmol})$ was added to a stirred solution of $\mathbf{5 9}(200 \mathrm{mg}, 0.48 \mathrm{mmol})$ containing 2,6-lutidine $(223 \mu \mathrm{L}, 205.73 \mathrm{mg}, 1.92 \mathrm{mmol})$, and 4-dimethylaminopyridine (DMAP; $117.3 \mathrm{mg}, 0.96 \mathrm{mmol})$ in $\mathrm{CH}_{2} \mathrm{Cl}_{2}(10 \mathrm{~mL})$ at room temperature. The resulting mixture was stirred at $35^{\circ} \mathrm{C}$ for $6 \mathrm{~h}$. The reaction mixture was then diluted with $\mathrm{CH}_{2} \mathrm{Cl}_{2}$ (40 mL) and partitioned with $\mathrm{H}_{2} \mathrm{O}$, and the aqueous layer was extracted with $\mathrm{CH}_{2} \mathrm{Cl}_{2}(2 \times$ $15 \mathrm{~mL})$. The organic layer was sequentially washed with $1 \mathrm{M} \mathrm{HCl}(15 \mathrm{~mL})$, saturated $\mathrm{NaHCO}_{3} / \mathrm{H}_{2} \mathrm{O}(15 \mathrm{~mL})$, and brine $(15 \mathrm{~mL})$, dried over $\mathrm{Na}_{2} \mathrm{SO}_{4}$, and evaporated. The resulting residue was column chromatographed $\left(0 \rightarrow 2 \% \mathrm{MeOH} / \mathrm{CHCl}_{3}\right)$ to give $\mathbf{6 0}{ }^{206}$ (230 $\mathrm{mg}, 78 \%)$ as a mixture of rotamers $(7: 3)$. The major rotamer had: ${ }^{1} \mathrm{H} \mathrm{NMR}\left(\mathrm{CDCl}_{3}\right) \delta$ 1.16-1.41 (m, 12H, $\left.6 \times \mathrm{CH}_{2}\right), 1.49-1.76\left(\mathrm{~m}, 2 \mathrm{H}, \mathrm{CH}_{2}\right), 1.93-2.07\left(\mathrm{~m}, 2 \mathrm{H}, \mathrm{CH}_{2}\right), 3.29$ (t, $J$ $\left.=7.1 \mathrm{~Hz}, 2 \mathrm{H}, \mathrm{NH}-\mathrm{CH}_{2}\right), 4.48-4.58\left(\mathrm{~m}, 1 \mathrm{H}, \mathrm{H} 4^{\prime}\right), 4.62-4.82\left(\mathrm{~m}, 2 \mathrm{H}, \mathrm{H} 5^{\prime}, 5^{\prime \prime}\right), 4.89-5.03(\mathrm{~m}$, 2H, $\left.\mathrm{CH}_{2}\right), 5.57-5.64(\mathrm{~m}, 1 \mathrm{H}, \mathrm{H} 3$ '), $5.73(\mathrm{~d}, J=7.6 \mathrm{~Hz}, 1 \mathrm{H}, \mathrm{H} 5), 5.80$ (tdd, $J=6.7,10.3$, 17.0 Hz, 1H, CH), 6.49-6.56 (m, 1H, H1'), 7.33 (dd, J = 0.6, 7.3 Hz, 1H, H6), 7.42-7.52 (m, 4H, Ar), 7.57-7.68 (m, 2H, Ar), 8.03-8.13 (m, 4H, Ar); ${ }^{13} \mathrm{C}$ NMR $\delta$ 26.67, 28.65, 28.87, 29.02, 29.28, 29.34, 29.69, 33.78, 43.97, 62.55 (C5'), 71.35 (dd, $J=17.1,35.2$ Hz, C3'), 79.57 (C4'), 83.54 (br s, C1'), 91.04 (C5), 114.17, 120.93 (t, $J=263.1 \mathrm{~Hz}, \mathrm{C} 2$ '), 126.78, $128.48,128.73,128.83,129.36,129.63,129.72,130.17,133.58,134.50,139.16$ (C6), 157.82 (C2), 165.99 (C4), 171.67; ${ }^{19} \mathrm{~F}$ NMR $\delta-120.35$ (br d, $\left.J=203.2 \mathrm{~Hz}, 1 \mathrm{~F}\right),-115.31$ (br d, $J=246.5 \mathrm{~Hz}, 1 \mathrm{~F}$ ). HRMS (ESI+) $m / z$ calcd for $\mathrm{C}_{34} \mathrm{H}_{40} \mathrm{~F}_{2} \mathrm{~N}_{3} \mathrm{O}_{6}[\mathrm{M}+\mathrm{H}]^{+}, 624.2880$; found 624.1860 . 
The minor rotamer had: ${ }^{1} \mathrm{H}$ NMR $\left(\mathrm{CDCl}_{3}\right) \delta$ 6.61-6.68 (m, 1H, H1'), 3.47-3.54 (m, 2H, $\mathrm{NH}-\mathrm{CH}_{2}$ ).

\section{4- $N$-(10,11-Dihydroxyundecanyl)-3',5'-di- $O$-benzoyl-2' -deoxy-2',2'-}

difluorocytidine (61). $\mathrm{OsO}_{4}(4 \mathrm{wt} \%$ in water; $0.016 \mathrm{mmol}, 4.02 \mathrm{mg}, 100 \mu \mathrm{L})$ and $\mathrm{N}$ methylmorpholine- $N$-oxide $(56.2 \mathrm{mg}, 0.48 \mathrm{mmol})$ were added to a stirred solution of $\mathbf{6 0}$ $(200 \mathrm{mg}, 0.32 \mathrm{mmol})$ in a mixture of acetone $(3 \mathrm{~mL})$ and water $(0.3 \mathrm{~mL})$ at $0{ }^{\circ} \mathrm{C}$. The resulting mixture was stirred at ambient temperature for $2 \mathrm{~h}$, and then diluted with $\mathrm{CH}_{2} \mathrm{Cl}_{2}$ (30 mL) and partitioned with $\mathrm{H}_{2} \mathrm{O}$. The organic layer was washed with saturated $\mathrm{NaHCO}_{3} / \mathrm{H}_{2} \mathrm{O}(15 \mathrm{~mL})$ and brine $(15 \mathrm{~mL})$, dried over $\mathrm{Na}_{2} \mathrm{SO}_{4}$, and evaporated. The resulting residue was column chromatographed $\left(50 \rightarrow 90 \%\right.$ EtOAc/hexane) to give $\mathbf{6 1}^{206}$ (202 mg, 96\%) as a mixture of isomers (7:3). The major isomer had: ${ }^{1} \mathrm{H}$ NMR $\left(\mathrm{CDCl}_{3}\right) \delta$ 1.21-1.37 (m, 12H, $\left.6 \times \mathrm{CH}_{2}\right), 1.38-1.45\left(\mathrm{~m}, 2 \mathrm{H}, \mathrm{CH}_{2}\right), 1.52-1.63\left(\mathrm{~m}, 2 \mathrm{H}, \mathrm{CH}_{2}\right), 3.40-3.48$ (m, 2H, NH-CH 2$), 3.61-3.66$ (m, 1H, CH), 3.68-3.73 (m, 1H, $\left.\mathrm{CH}_{2}\right), 4.49-4.55\left(\mathrm{~m}, 1 \mathrm{H}, \mathrm{H} 4^{\prime}\right)$, $4.70\left(\mathrm{dd}, J=4.6,12.2 \mathrm{~Hz}, 1 \mathrm{H}, \mathrm{H} 5^{\prime}, 5^{\prime \prime}\right), 5.5-5.67$ (m, 1H, H3'), 5.71 (d, $J=7.6 \mathrm{~Hz}, 1 \mathrm{H}$, H5), 6.55-6.62 (m, 1H, H1'), 7.32 (d, $J=7.0 \mathrm{~Hz}, 1 \mathrm{H}, \mathrm{H6}), 7.39-7.51$ (m, 4H, Ar), 7.527.67 (m, 2H, Ar), 8.01-8.10 (m, 4H, Ar); ${ }^{13} \mathrm{C}$ NMR $\delta$ 25.28, 26.41, 26.61, 28.57, 28.70, $28.85,29.19,33.02,43.70,62.55\left(\mathrm{C}^{\prime}\right), 66.85,71.61\left(\mathrm{~m}, \mathrm{C} 3^{\prime}\right), 72.22,77.81,79.25,91.34$ (C5), $121.14\left(\mathrm{t}, J=263.1 \mathrm{~Hz}, \mathrm{C} 2{ }^{\prime}\right), 127.64,128.70,128.80,129.07,129.71,130.15,133.56$, $133.72,134.17,134.43,158.82,164.79,165.97 ;{ }^{19} \mathrm{~F}$ NMR $\delta-120.42$ (br d, $J=243.4 \mathrm{~Hz}$, $1 \mathrm{~F}),-115.30$ (br d, $J=260.1 \mathrm{~Hz}, 1 \mathrm{~F}) . \mathrm{HRMS}(\mathrm{ESI}+) \mathrm{m} / z$ calcd for $\mathrm{C}_{34} \mathrm{H}_{42} \mathrm{~F}_{2} \mathrm{~N}_{3} \mathrm{O}_{8}[\mathrm{M}+\mathrm{H}]^{+}$, 658.2934; found 658.2930.

The minor isomer had: ${ }^{1} \mathrm{H}$ NMR $\left(\mathrm{CDCl}_{3}\right) \delta$ 6.48-6.55 (m, $\left.1 \mathrm{H}, \mathrm{H} 1{ }^{\prime}\right), 3.22-3.28(\mathrm{~m}, 2 \mathrm{H}, \mathrm{NH}-$ $\left.\mathrm{CH}_{2}\right)$. 


\section{4- $N$-[10-Hydroxy-11-(4-methylphenylsulfoxy)undecanyl]-3',5'-di- $O$-benzoyl-2'-}

deoxy-2',2'-difluorocytidine (62). $\mathrm{TsCl}(29.6 \mathrm{mg}, 0.15 \mathrm{mmol})$ was added to a stirred solution of $\mathbf{6 1}(100 \mathrm{mg}, 0.15 \mathrm{mmol})$ in anhydrous $\mathrm{CH}_{2} \mathrm{Cl}_{2}(4 \mathrm{~mL})$ in the presence of $\mathrm{Et}_{3} \mathrm{~N}$ (32 $\mu \mathrm{L}, 23 \mathrm{mg}, 0.23 \mathrm{mmol}$ ). The mixture was then heated at $40{ }^{\circ} \mathrm{C}$ for 15 hours. After cooling, the reaction mixture was diluted with $\mathrm{CH}_{2} \mathrm{Cl}_{2}$ and partitioned with $\mathrm{HCl}(0.1 \mathrm{M})$ and $\mathrm{H}_{2} \mathrm{O}$. The organic layer was washed with saturated $\mathrm{NaHCO}_{3} / \mathrm{H}_{2} \mathrm{O}(15 \mathrm{~mL})$ and brine $\left(15 \mathrm{~mL}\right.$ ), dried over $\mathrm{Na}_{2} \mathrm{SO}_{4}$, and evaporated. The resulting residue was column chromatographed ( $20 \rightarrow 50 \%$ EtOAc/hexane) to give $\mathbf{6 2}^{206}(76 \mathrm{mg}, 62 \%)$ as a mixture of isomers (6:4). The major isomer had: ${ }^{1} \mathrm{H}$ NMR $\left(\mathrm{CDCl}_{3}\right) \delta 1.21-1.31\left(\mathrm{~m}, 12 \mathrm{H}, 6 \times \mathrm{CH}_{2}\right)$, $1.40-1.47\left(\mathrm{~m}, 2 \mathrm{H}, \mathrm{CH}_{2}\right), 1.55-1.68\left(\mathrm{~m}, 2 \mathrm{H}, \mathrm{CH}_{2}\right), 2.46\left(\mathrm{~s}, 3 \mathrm{H}, \mathrm{CH}_{3}\right), 3.48-3.55(\mathrm{~m}, 2 \mathrm{H}, \mathrm{NH}-$ $\left.\mathrm{CH}_{2}\right), 3.82-3.88(\mathrm{~m}, 1 \mathrm{H}, \mathrm{CH}), 3.89-3.95(\mathrm{~m}, 1 \mathrm{H}, \mathrm{CH}), 4.05(\mathrm{dd}, J=2.9,9.8 \mathrm{~Hz}, 1 \mathrm{H}, \mathrm{CH})$, 4.53-4.64 (m, 1H, H4'), $4.68\left(\mathrm{dd}, J=4.4,12.2 \mathrm{~Hz}, 1 \mathrm{H}, \mathrm{H} 5^{\prime}\right), 4.81(\mathrm{dd}, J=3.4,12.3 \mathrm{~Hz}$, 1H, H5"), 5.38-5.45 (m, 1H, H3'), 5.61 (d, $J=7.6 \mathrm{~Hz}, 1 \mathrm{H}, \mathrm{H} 5), 6.63-6.70$ (m, 1H, H1'), 7.31-7.39 (m, 3H, H6, Ar), 7.42-7.54 (m, 4H, Ar), 7.59-7.68 (m, 2H, Ar), 7.73 (d, $J=8.3$ $\mathrm{Hz}, 2 \mathrm{H}, \mathrm{Ar}), 8.05-8.12$ (m, 4H, Ar); ${ }^{13} \mathrm{C}$ NMR $\delta 21.80,25.21,26.87,29.32,29.35,29.41$, 29.58, 29.85, 32.77, 41.17, 63.03 (C5'), 69.62, 71.15 (m, C3'), 74.14, 96.02 (C5), 121.19 (t, $\left.J=262.8 \mathrm{~Hz}, \mathrm{C} 2^{\prime}\right), 128.11,128.75,128.89,129.45,129.49,129.86,130.10,130.33$, $132.91,133.65,134.32,145.19,164.85,165.09,166.11 ;{ }^{19} \mathrm{~F}$ NMR $\delta-120.51$ (br d, $J=$ $241.2 \mathrm{~Hz}, 1 \mathrm{~F}),-115.34$ (d, $J=246.8 \mathrm{~Hz}, 1 \mathrm{~F})$.

The minor isomer had: ${ }^{1} \mathrm{H}$ NMR $\left(\mathrm{CDCl}_{3}\right) \delta 6.53-6.60\left(\mathrm{~m}, 1 \mathrm{H}, \mathrm{H} 1^{\prime}\right), 3.26-3.33(\mathrm{~m}, 2 \mathrm{H}, \mathrm{NH}-$ $\left.\mathrm{CH}_{2}\right)$.

4- $N$-[11-(4-Methylphenylsulfoxy)-10-oxoundecanyl]-3',5'-di- $O$-benzoyl-2'-deoxy2',2'-difluorocytidine (56). A freshly prepared solution of Collins reagent $\left[\mathrm{CrO}_{3}(24.6 \mathrm{mg}\right.$, 
$0.246 \mathrm{mmol}$ ), pyridine (5 $\mu \mathrm{L}, 24.6 \mathrm{mg}, 0.246 \mathrm{mmol})$, and $\mathrm{Ac}_{2} \mathrm{O}(45 \mu \mathrm{L}, 51 \mathrm{mg}, 0.493$ mmol) in $\left.\mathrm{CH}_{2} \mathrm{Cl}_{2}(2 \mathrm{~mL})\right]$ was added to a stirred solution of $62(50 \mathrm{mg}, 0.062 \mathrm{mmol})$ in $\mathrm{CH}_{2} \mathrm{Cl}_{2}(4 \mathrm{~mL})$ at ambient temperature. The resulting mixture was stirred for $1 \mathrm{~h}$. and was immediately column chromatographed (EtOAc) to give $\mathbf{5 6}^{206}$ (47 mg, 94\%). The major (6:4) rotamer had: ${ }^{1} \mathrm{H}$ NMR $\left(\mathrm{CDCl}_{3}\right) \delta 1.20-1.38\left(\mathrm{~m}, 12 \mathrm{H}, 6 \times \mathrm{CH}_{2}\right), 1.41-1.65(\mathrm{~m}, 2 \mathrm{H}$, $\left.\mathrm{CH}_{2}\right), 2.44\left(\mathrm{~s}, 3 \mathrm{H}, \mathrm{CH}_{3}\right), 2.45-2.51\left(\mathrm{~m}, 2 \mathrm{H}, \mathrm{CH}_{2}\right), 3.46-3.53\left(\mathrm{~m}, 2 \mathrm{H}, \mathrm{NH}-\mathrm{CH}_{2}\right), 4.48(\mathrm{~s}, 2 \mathrm{H}$, $\left.\mathrm{CH}_{2}\right), 4.52-4.62\left(\mathrm{~m}, 1 \mathrm{H}, \mathrm{H} 4^{\prime}\right), 4.67\left(\mathrm{dd}, J=4.4,12.1 \mathrm{~Hz}, 1 \mathrm{H}, \mathrm{H} 5^{\prime}\right), 4.79(\mathrm{dd}, J=3.4,12.2$ Hz, 1H, H5"), 5.26-5.31 (m, 1H, H3'), 5.58 (d, $J=7.6 \mathrm{~Hz}, 1 \mathrm{H}, \mathrm{H} 5), 6.60-6.68$ (m, 1H, H1'), 7.32-7.38 (m, 3H, H6, Ar), 7.41-7.51 (m, 4H, Ar), 7.57-7.66 (m, 2H, Ar), 7.81 (d, $J$ $=8.3 \mathrm{~Hz}, 2 \mathrm{H}, \mathrm{Ar}), 8.03-8.12(\mathrm{~m}, 4 \mathrm{H}, \mathrm{Ar}) ;{ }^{13} \mathrm{C}$ NMR $\delta 21.72,22.77,26.77,28.82,28.99$, 29.00, 29.08, 29.11 38.93, 41.01, $62.91\left(\mathrm{C}^{\prime}\right), 71.53$ (dd, $\left.J=17.2,35.4 \mathrm{~Hz}, \mathrm{C} 3^{\prime}\right), 71.83$, 77.36 (C4'), 84.01 (br s, C1'), 96.17 (C5), 120.90 (t, $J=263.1 \mathrm{~Hz}, \mathrm{C} 2$ '), 125.99, 128.72, $128.82,128.94,129.38,129.41,129.45,129.57,130.03,130.54,130.86,130.91,133.16$, 134.28, 134.35, 135.12, 135.24, 140.69 (C6), 146.42 (C4), 156.51, 164.39, 165.88, 166.99, 203.43; ${ }^{19} \mathrm{~F}$ NMR $\delta-120.69$ (br d, $\left.J=252.5 \mathrm{~Hz}, 1 \mathrm{~F}\right),-115.28$ (d, $\left.J=248.2 \mathrm{~Hz}, 1 \mathrm{~F}\right)$. HRMS (ESI+) $m / z$ calcd for $\mathrm{C}_{41} \mathrm{H}_{46} \mathrm{~F}_{2} \mathrm{~N}_{3} \mathrm{O}_{10} \mathrm{~S}[\mathrm{M}+\mathrm{H}]^{+}, 810.2866$; found 810.2891.

The minor rotamer had: ${ }^{1} \mathrm{H}$ NMR $\left(\mathrm{CDCl}_{3}\right) \delta$ 6.51-6.58 (m, 1H, H1'), 3.23-3.31 (m, 2H, $\mathrm{NH}-\mathrm{CH}_{2}$ ).

\section{4- $N$-(11-Fluoro-10-oxoundecanyl)-3',5'-di- $O$-benzoyl-2'-deoxy-2',2'-}

difluorocytidine (63). Tosylate derivative 56 (30 $\mathrm{mg}, 0.03 \mathrm{mmol}$ ) was treated with KF (8.4 $\mathrm{mg}, 0.15 \mathrm{mmol}), \mathrm{K}_{2} \mathrm{CO}_{3}(19.5 \mathrm{mg}, 0.15 \mathrm{mmol}), 18$-Crown-6 (38.1 mg, $\left.0.15 \mathrm{mmol}\right)$ in $\mathrm{CH}_{3} \mathrm{CN}(3 \mathrm{~mL})$ with stirring for $1 \mathrm{~h}$ at $65^{\circ} \mathrm{C}$. The reaction mixture was quickly cooled in a water bath and filtered. The solvent was evaporated under vacuum, and the resulting 
residue was column chromatographed $\left(20 \rightarrow 40 \%\right.$ EtOAc/hexane) to give $\mathbf{6 3}^{206}(8 \mathrm{mg}$, $38 \%)$, as a mixture of rotamers (6:4), and unchanged $\mathbf{5 6}(\sim 50 \%)$. A practical yield of $76 \%$

was achieved for the conversion of $\mathbf{5 6}$ to $\mathbf{6 3}$. The major rotamer had: ${ }^{1} \mathrm{H} \mathrm{NMR}\left(\mathrm{CDCl}_{3}\right) \delta$ 1.19-1.38 (m, $\left.12 \mathrm{H}, 6 \times \mathrm{CH}_{2}\right), 1.55-1.78\left(\mathrm{~m}, 2 \mathrm{H}, \mathrm{CH}_{2}\right), 2.55\left(\mathrm{t}, J=7.3 \mathrm{~Hz}, 2 \mathrm{H}, \mathrm{CH}_{2}\right), 3.33-$ $3.41\left(\mathrm{~m}, 2 \mathrm{H}, \mathrm{NH}-\mathrm{CH}_{2}\right), 4.54-4.65\left(\mathrm{~m}, 1 \mathrm{H}, \mathrm{H} 4^{\prime}\right), 4.76$ (dd, $\left.J=4.6,12.3 \mathrm{~Hz}, 1 \mathrm{H}, \mathrm{H} 5^{\prime}\right), 4.79$ (d, $\left.J=47.7 \mathrm{~Hz}, 2 \mathrm{H}, \mathrm{CH}_{2} \mathrm{~F}\right), 4.81\left(\mathrm{dd}, J=3.6,12.1 \mathrm{~Hz}, 1 \mathrm{H}, \mathrm{H} 5^{\prime \prime}\right), 5.59(\mathrm{~d}, J=7.6 \mathrm{~Hz}, 1 \mathrm{H}$, H5), 5.85-5.92 (m, 1H, H3'), 6.42-6.48 (m, 1H, H1'), 7.43-7.55 (m, 4H, Ar), 7.56-7.69 (m, 2H, Ar), 7.76 (d, $J=7.3 \mathrm{~Hz}, 1 \mathrm{H}, \mathrm{H} 6), 8.01-8.12(\mathrm{~m}, 4 \mathrm{H}, \mathrm{Ar}) ;{ }^{13} \mathrm{C}$ NMR $\delta 22.72,26.69$, $28.68,28.97,29.06,29.21,29.85,38.30,38.32,38.41,44.03,62.66\left(\mathrm{C}^{\prime}\right), 71.65$ (dd, $J=$ 17.2, 35.4 Hz, C3'), 79.38 (C4'), 83.91 (br s, C1'), 85.04 (d, $\left.J=185.2 \mathrm{~Hz}, \mathrm{CH}_{2} \mathrm{~F}\right), 91.13$ (C5), 120.67 (t, $J=263.1 \mathrm{~Hz}, \mathrm{C} 2$ '), 127.62, 128.89, 128.98, 129.12, 129.85, 130.31, 133.77, $133.45,134.39,134.67,137.68$ (C6), 145.20 (C4), 158.00, 164.93, 166.11, 203.34; ${ }^{19} \mathrm{~F}$ NMR $\delta-227.42(\mathrm{t}, J=47.7,1 \mathrm{~F}),-120.37(\mathrm{br} \mathrm{d}, J=217.9 \mathrm{~Hz}, 1 \mathrm{~F}),-115.21(\mathrm{dd}, J=245.1$ $\mathrm{Hz}, 1 \mathrm{~F}$ ). HRMS (ESI+) $\mathrm{m} / z$ calcd for $\mathrm{C}_{34} \mathrm{H}_{39} \mathrm{~F}_{3} \mathrm{~N}_{3} \mathrm{O}_{7}[\mathrm{M}+\mathrm{H}]^{+}, 658.2747$; found, 658.2745.

The minor rotamer had: ${ }^{1} \mathrm{H}$ NMR $\left(\mathrm{CDCl}_{3}\right) \delta$ 6.55-6.64 (m, 1H, H1'), 3.51-3.58 (m, $2 \mathrm{H}$, $\left.\mathrm{NH}-\mathrm{CH}_{2}\right)$.

\section{4- $N$-(11-Fluoro-10-oxoundecanyl)-2'-deoxy-2',2'-difluoro-cytidine}

(64).

Compound 63 (20 mg, $0.03 \mathrm{mmol})$ was dissolved in methanolic ammonia $(2 \mathrm{~mL})$ at $0{ }^{\circ} \mathrm{C}$, then stirred at ambient temperature for $2 \mathrm{~h}$. Volatiles were evaporated under reduced pressure, and the resulting residue was chromatographed $\left(0 \rightarrow 5 \% \mathrm{MeOH} / \mathrm{CHCl}_{3}\right)$ to give 64 $^{206}(7 \mathrm{mg}, 52 \%)$ as a clear oil: ${ }^{1} \mathrm{H}$ NMR $\left(\mathrm{MeOD}-d_{4}\right) \delta 1.22-1.35\left(\mathrm{~m}, 12 \mathrm{H}, 6 \times \mathrm{CH}_{2}\right)$, 1.47-1.62 (m, 2H, $\left.\mathrm{CH}_{2}\right), 2.43\left(\mathrm{t}, J=7.3 \mathrm{~Hz}, 2 \mathrm{H}, \mathrm{CH}_{2}\right), 3.30-3.42\left(\mathrm{~m}, 2 \mathrm{H}, \mathrm{NH}-\mathrm{CH}_{2}\right), 3.69-$ $3.75\left(\mathrm{~m}, 1 \mathrm{H}, \mathrm{H} 4^{\prime}\right), 3.85\left(\mathrm{dd}, J=4.6,12.3 \mathrm{~Hz}, 1 \mathrm{H}, \mathrm{H} 5^{\prime}\right), 3.95(\mathrm{dd}, J=3.6,12.1 \mathrm{~Hz}, 1 \mathrm{H}$, 
$\left.\mathrm{H}^{\prime \prime}\right), 4.19-4.28(\mathrm{~m}, 1 \mathrm{H}, \mathrm{H} 3 '), 4.79$ (d, $\left.J=47.7 \mathrm{~Hz}, 2 \mathrm{H}, \mathrm{CH}_{2} \mathrm{~F}\right), 5.92(\mathrm{~d}, J=7.9 \mathrm{~Hz}, 1 \mathrm{H}$, H5), 6.08-6.15 (m, 1H, H1'), 7.98 (d, $J=7.3 \mathrm{~Hz}, 1 \mathrm{H}, \mathrm{H} 6) ;{ }^{13} \mathrm{C}$ NMR $\delta 23.88,27.94,29.94$, 30.17, 30.31, 30.72, 38.55, 41.70, $60.56\left(\mathrm{C}^{\prime}\right), 71.43\left(\mathrm{~m}, \mathrm{C} 3{ }^{\prime}\right), 82.22\left(\mathrm{C}^{\prime}\right), 85.04(\mathrm{~d}, J=$ 185.2 Hz, $\mathrm{CH}_{2} \mathrm{~F}$ ), 87.96 (C1'), 97.36 (C5), 124.00 ("m", C2'), 140.77 (C6), 158.29 (C2), 165.35 (C4), 202.21; ${ }^{19} \mathrm{~F}$ NMR $\delta-227.44(\mathrm{t}, J=47.7 \mathrm{~Hz}, 1 \mathrm{~F}),-119.98$ (d of m, $J=239.1$ $\mathrm{Hz}, 1 \mathrm{~F}),-119.75$ (d of m, $J=244.1 \mathrm{~Hz}, 1 \mathrm{~F}$ ). HRMS (ESI+) $m / z$ calcd for $\mathrm{C}_{20} \mathrm{H}_{30} \mathrm{~F}_{3} \mathrm{~N}_{3} \mathrm{O}_{5}$ $[\mathrm{M}+\mathrm{H}]^{+}, 450.2210 ;$ found 450.2244 .

Note: Treatment of $56(15 \mathrm{mg}, 0.019 \mathrm{mmol})$ with $\mathrm{KF}$ (4 eq.) in the presence of Kryptofix 2.2.2. (4 eq.), rather than $18-\mathrm{Crown}-6$, in $\mathrm{CH}_{3} \mathrm{CN}$ at $75^{\circ} \mathrm{C}$ for $30 \mathrm{~min}$ followed by a quick filtration and evaporation of the mother liquor gave oily residue containing 63 . Immediate treatment $(45 \mathrm{~min} / \mathrm{rt})$ of this crude mixture with $\mathrm{NH}_{3} / \mathrm{MeOH}(2 \mathrm{~mL})$ gave a complex mixture with product 64 in low yields ( 5\%; based on TLC and crude NMRs).

\subsubsection{Synthesis of 4- $N$-alkylgemcitabine NOTA ${ }^{68} \mathrm{Ga}$ chelators}

\section{4- $N$-[3- $N$-(tert-Butoxycarbonyl)-3-aminopropanyl]-2'-deoxy-2',2'-}

difluorocytidine (66). In a tightly sealed vessel, mixture of $\mathbf{5 8 a}^{\mathbf{3 3}}(11.5 \mathrm{mg}, 0.018 \mathrm{mmol})$ and commercially available $N$-Boc-1,3-propanediamine $(150 \mu \mathrm{l}, 150 \mathrm{mg}, 0.86 \mathrm{mmol})$ in 1,4-dioxane was stirred at $65^{\circ} \mathrm{C}$. After $48 \mathrm{~h}$, the volatiles were evaporated the resulting residue was column chromatographed $(30 \% \mathrm{EtOAc} /$ hexane $\rightarrow 5 \% \mathrm{MeOH} / \mathrm{EtOAc})$ to give fully protected 3',5'-di-O-Boc-4- $N$-(3-N'-Boc-3-aminopropanyl)gemcitabine 66a [3.6 mg, $31 \%$; MS (ESI+) m/z $621(100,[\mathrm{M}+\mathrm{H}]+)]$ followed by partially protected $\mathbf{6 6}^{207}(3.5 \mathrm{mg}$, 59\%) as colorless oil: ${ }^{1} \mathrm{H}$ NMR $\delta 1.43\left(\mathrm{~s}, 9 \mathrm{H}, 3 \times \mathrm{CH}_{3}\right), 1.74$ (“quint”, $J=6.8 \mathrm{~Hz}, 2 \mathrm{H}, \mathrm{CH}_{2}$ ), $3.10\left(\mathrm{t}, J=6.7 \mathrm{~Hz}, 2 \mathrm{H}, \mathrm{CH}_{2}\right), 3.41\left(\mathrm{t}, J=6.9 \mathrm{~Hz}, 2 \mathrm{H}, \mathrm{CH}_{2}\right), 3.77(\mathrm{dd}, J=3.2 \mathrm{~Hz}, 12.6 \mathrm{~Hz}$, 1H, H5'), 3.87 (dt, $J=2.8,8.3 \mathrm{~Hz}, 1 \mathrm{H}, \mathrm{H} 4$ '), 3.93 (“d”, $\left.J=2.1,12.6 \mathrm{~Hz}, 1 \mathrm{H}, \mathrm{H} 5^{\prime \prime}\right), 4.24$ 
(dt, $J=8.4,12.1 \mathrm{~Hz}, 1 \mathrm{H}, \mathrm{H} 3$ '), 5.85 (d, $J=7.6 \mathrm{~Hz}, 1 \mathrm{H}, \mathrm{H} 5), 6.21$ (“'t”, $J=8.0 \mathrm{~Hz}, 1 \mathrm{H}, \mathrm{H1}$ '), 7.75 (d, $J=7.6 \mathrm{~Hz}, 1 \mathrm{H}, \mathrm{H} 6) ;{ }^{19} \mathrm{~F}$ NMR $\delta-119.94$ (br. d, $\left.J=240.6 \mathrm{~Hz}, 1 \mathrm{~F}\right),-118.83$ (dd, $J$ $=10.3,238.3 \mathrm{~Hz}, 1 \mathrm{~F}) ; \mathrm{MS}(\mathrm{ESI}) \mathrm{m} / \mathrm{z} 421(100,[\mathrm{M}+\mathrm{H}]+)$.

\section{4- $N$-(3-Aminopropanyl)-2'-deoxy-2',2'-difluorocytidine (67). Method A.} Compound 66 (3.4 mg, $0.008 \mathrm{mmol})$ was dissolved in TFA $(1.0 \mathrm{~mL})$ and the mixture was stirred at ambient temperature overnight. After $15 \mathrm{~h}$, the reaction mixture was diluted with toluene, the volatiles were evaporated, and the residue co-evaporated with a fresh portion of toluene. The resulting residue was then column chromatographed [EtOAc $\rightarrow 50 \%$ EtOAc/SSE (EtOAc/i-PrOH/ $\mathrm{H}_{2} \mathrm{O}, 4: 2: 1$; upper layer)] to give $\mathbf{6 7}^{207}(2.4 \mathrm{mg}, 93 \%)$ as a white solid: ${ }^{1} \mathrm{H}$ NMR $\delta 1.95$ ("quint", $J=6.8 \mathrm{~Hz}, 2 \mathrm{H}, \mathrm{CH}_{2}$ ), 3.19 (t, $J=6.8 \mathrm{~Hz}, 2 \mathrm{H}, \mathrm{CH}_{2}$ ), 3.51-3.57 (m, 2H, $\mathrm{CH}_{2}$ ), 3.81 (dd, $J=3.1,12.0 \mathrm{~Hz}, 1 \mathrm{H}, \mathrm{H} 5$ '), 3.91-3.99 (m, 2H, H4', H5"), 4.28 (dt, $J=8.2,12.1 \mathrm{~Hz}, 1 \mathrm{H}, \mathrm{H} 3$ '), $5.92(\mathrm{~d}, J=7.5 \mathrm{~Hz}, 1 \mathrm{H}, \mathrm{H} 5), 6.25$ ("t," $J=8.1 \mathrm{~Hz}, 1 \mathrm{H}$, H1'), $7.88(\mathrm{~d}, J=7.1 \mathrm{~Hz}, 1 \mathrm{H}, \mathrm{H} 6) ;{ }^{13} \mathrm{C}$ NMR $\delta 34.7,38.6,39.1,60.5(\mathrm{C} 5$ '), 70.1 (dd, $J=$ 21.0, 24.2 Hz, C3'), 82.8 (dd, $\left.J=4.1,5.1 \mathrm{~Hz}, \mathrm{C}^{\prime}\right), 86.2(\mathrm{C} 1), 97.8$ (C5), 125.0 (t, $J=256.8$ Hz, C2'), 140.1 (C6), 158.9 (C2), 165.9 (C4); ${ }^{19}$ F NMR $\delta$-118.92 (br. d, $J=241.2 \mathrm{~Hz}, 1 \mathrm{~F}$ ), $-120.11(\mathrm{dd}, J=10.4,237.8 \mathrm{~Hz}, 1 \mathrm{~F}) ; \mathrm{HRMS}\left(\mathrm{ESI}^{+}\right) \mathrm{m} / z$ calcd for $\mathrm{C}_{12} \mathrm{H}_{19} \mathrm{~F}_{2} \mathrm{~N}_{4} \mathrm{O}_{4}[\mathrm{M}+\mathrm{H}]^{+}$, 321.1369, found 321.1346 .

Method B. In a tightly sealed container, a solution of $\mathbf{5 8}^{33}(19.6 \mathrm{mg}, 0.048 \mathrm{mmol})$, commercially available $N$-Boc-1,3-propanediamine $(100 \mu \mathrm{L}, 100 \mathrm{mg}, 0.58 \mathrm{mmol})$ and TEA ( $3 \mathrm{~mL}$ ) in 1,4-dioxane was stirred at $75^{\circ} \mathrm{C}$. After $96 \mathrm{~h}$, the volatiles were evaporated and the resulting residue was treated with TFA $(1.5 \mathrm{~mL})$ and stirred at ambient temperature overnight. After $15 \mathrm{~h}$, the reaction mixture was diluted with toluene, the volatiles were evaporated, and the residue co-evaporated with a fresh portion of toluene. The resulting 
residue was then column chromatographed (10\% $\mathrm{MeOH} / \mathrm{EtOAc})$ to give $\mathbf{6 7}^{207}$ (15.6 mg, 94\%) with data as reported above.

\section{4- $N$-[3- $N$-(SCN-Bn-NOTA)aminopropanyl]-2'-deoxy-2',2'-difluorocytidine (65).}

A mixture of SCN-Bn-NOTA (10 mg, $0.031 \mathrm{mmol})$ and $67(10 \mathrm{mg}, 0.018 \mathrm{mmol})$ in $3 \mathrm{~mL}$ of $0.1 \mathrm{M} \mathrm{Na}_{2} \mathrm{CO}_{3}$ buffer ( $\mathrm{pH} \mathrm{11)}$ was stirred at ambient temperature for $60 \mathrm{~h}$. The reaction mixture was injected into a Phenomenex Gemini semi-preparative RP-C18 column ( $5 \mu, 25$ $\mathrm{cm} \times 1 \mathrm{~cm}$ ) via $2 \mathrm{~mL}$ loop and eluted with $0 \% \rightarrow 100 \%$ ethanol gradient in $0.01 \% \mathrm{TFA} / \mathrm{H}_{2} \mathrm{O}$ from 0 to $30 \mathrm{~min}$ at a flow rate $=3 \mathrm{~mL} / \mathrm{min}$. Product $65(12.5 \mathrm{mg}, 54 \%$ yield $)$ eluted with $\mathrm{rt}=12.8 \mathrm{~min}:{ }^{1} \mathrm{H}$ NMR $\delta 1.90$ ("quint", $\left.J=6.5 \mathrm{~Hz}, 2 \mathrm{H}, \mathrm{CH}_{2}\right), 2.68-2.75(\mathrm{~m}, 2 \mathrm{H}), 2.87-2.95$ (m, 4H), 3.06-3.15 (m, 3H), 3.17-3.29 (m, 5H), 3.41-3.48 (m, 4H), 3.50-3.62 (m, 1H), 3.65$3.72(\mathrm{~m}, 4 \mathrm{H}), 3.79$ (dd, $J=3.2 \mathrm{~Hz}, 12.5 \mathrm{~Hz}, 1 \mathrm{H}, \mathrm{H} 5 "), 3.88-3.98$ (m, 2H, H4, H5'), 4.204.29 (m, 1H, H3'), 5.89 (d, $J=7.6 \mathrm{~Hz}, 1 \mathrm{H}, \mathrm{H} 5), 6.26$ ("t", $J=8.0 \mathrm{~Hz}, \mathrm{H} 1$ '), 7.22-7.28 (m, 2H, Ar), 7.31-7.37 (m, 2H, Ar), $7.79(\mathrm{~d}, J=7.6 \mathrm{~Hz}, 1 \mathrm{H}, \mathrm{H} 6) ;{ }^{19} \mathrm{~F}$ NMR $\delta-118.80$ (d of m, $J=242.1 \mathrm{~Hz}, 1 \mathrm{~F}),-119.45(\mathrm{~d}$ of $\mathrm{m}, J=244.1 \mathrm{~Hz}, 1 \mathrm{~F}) . \mathrm{MS}\left(\mathrm{ESI}^{+}\right) \mathrm{m} / z .771\left(100,[\mathrm{M}+\mathrm{H}]^{+}\right)$; HRMS $\left(\mathrm{ESI}^{+}\right) \mathrm{m} / \mathrm{z}$ calcd for $\mathrm{C}_{32} \mathrm{H}_{45} \mathrm{~F}_{2} \mathrm{~N}_{8} \mathrm{O}_{10} \mathrm{~S}[\mathrm{M}+\mathrm{H}]^{+} 771.2942$; found 771.2879.

\section{Gallium-4- $N$-[3-N-(SCN-Bn-NOTA)aminopropanyl]-2' -deoxy-2',2' -}

difluorocytidine (68). Gallium(III) chloride $(5.1 \mathrm{mg}, 0.029 \mathrm{mmol})$ was added to a stirred solution of $65(1.0 \mathrm{mg}, 0.001 \mathrm{mmol})$ in $0.6 \mathrm{~N} \mathrm{NaCH}_{3} \mathrm{CO}_{2} / \mathrm{H}_{2} \mathrm{O}(1 \mathrm{~mL}, \mathrm{pH}=9.3)$ at ambient temperature. After $30 \mathrm{~min}$, the reaction mixture was diluted to a total volume of $1.5 \mathrm{~mL}$ with $\mathrm{H}_{2} \mathrm{O}$ and injected into a Phenomenex Gemini semi-preparative RP-C18 column $(5 \mu$, $25 \mathrm{~cm} \times 1 \mathrm{~cm}$ ) via $2 \mathrm{~mL}$ loop and eluted with $0 \rightarrow 100 \%$ ethanol gradient in $0.01 \%$ TFA $/ \mathrm{H}_{2} \mathrm{O}$ from 0 to $30 \mathrm{~min}$ at a flow rate $=3 \mathrm{~mL} / \mathrm{min}$. Product $68(0.5 \mathrm{mg}, 46 \%$ yield $)$ 
eluted with $t_{\mathrm{R}}=11.5 \mathrm{~min}$. HRMS $\left(\mathrm{ESI}^{+}\right) \mathrm{m} / \mathrm{z}$ calcd for $\mathrm{C}_{32} \mathrm{H}_{42} \mathrm{~F}_{2} \mathrm{GaN}_{8} \mathrm{NaO}_{10} \mathrm{~S}[\mathrm{M}+\mathrm{H}]^{+}$ 837.1963; found 837.1977.

\subsubsection{1 ${ }^{68} \mathrm{Ga}$ radiolabeling and in vivo imaging of 4- $N$-alkyl gemcitabine-SCN-}

\section{Bn-NOTA}

${ }^{68} \mathrm{Ge}{ }^{68} \mathrm{Ga}$ Radioisotopic Generator: Two different ${ }^{68} \mathrm{Ge} /{ }^{68} \mathrm{Ga}$ generators were used in this study, a 50 mCi IGG-100 (Eckert \& Ziegler, Germany), based on the $\mathrm{TiO}_{2}$ resin technology; and a 50 mCi ITG generator (ItM, Germany), based on a modified silica resin.

Labeling with IGG-100 ${ }^{68} \mathrm{Ge}{ }^{68} \mathrm{Ga}$ Generator: The generator was eluted with $5 \mathrm{~mL}$ of 0.1 M ultrapure HCl (Sigma-Aldrich, USA) solution. Since the elution contains metal contaminants, a combination of chromatographic exchange resins were used for prepurification of the eluent as described previously. ${ }^{208}$ Briefly, two luer-fitting column beds were prepared. First, $40 \mathrm{mg}$ of AG-50Wx8 cation exchange (Eichrom, USA) column was connected to a three-way stopcock. Next, $15 \mathrm{mg}$ of UTEVA anion exchange (Eichrom, USA) resin in a column was positioned. Finally, another three-way stopcock was located at the end. The system was designed to be used in four simple steps: elution (from the generator, $5 \mathrm{~mL}$ of $0.1 \mathrm{M} \mathrm{HCl})$; cleaning $(1 \mathrm{~mL}$ of $0.1 \mathrm{M} \mathrm{HCl})$; purification $(1 \mathrm{~mL}$ of $5 \mathrm{M}$ $\mathrm{HCl}$ ); extraction (1 mL of Millipore Water).

The purified ${ }^{68} \mathrm{Ga}$ elution $(1 \mathrm{~mL}, \mathrm{pH}=0.6-1$; activity $=10-20 \mathrm{mCi})$ obtained from the purification system was collected in a $15 \mathrm{~mL}$ centrifuge tube. A solution containing $\mathbf{6 5}$ (10 $\mu \mathrm{g}$, or $10 \mu \mathrm{l}$ of a $1 \mathrm{mg} / \mathrm{ml}$ solution in DMSO) was later added and immediately buffered using $0.3 \mathrm{~mL}$ of $3 \mathrm{~N}$ ultrapure sodium acetate (Sigma-Aldrich, USA). Labeling was performed using a thermomixer stirring at $750 \mathrm{rpm}$ for 15 minutes at room temperature (Eppendorf, Germany), taking samples at 5,10 and 15 minutes of incubation. No further 
purification was done for initial testing. Final quality control was performed in Silicagel 60 TLC plates with $0.1 \mathrm{M}$ citric acid as the mobile phase. Plates were quantified in OptiQuant software (Perkin Elmer, USA) by exposing a phosphoscreen for 5 min and reading it in a Packard Cyclone Phosphorimager (Perkin Elmer, USA). This labeled product was not used for imaging.

Labeling with the ITG ${ }^{68} \mathrm{Ge}^{88} \mathrm{Ga}$ Generator: This generator was eluted using $4 \mathrm{ml}$ of $0.05 \mathrm{M} \mathrm{HCl}$ and collected in a $15 \mathrm{ml}$ sterile centrifuge tube. The obtained solution containing up to $1850 \mathrm{MBq}(50 \mathrm{mCi})$ of ${ }^{68} \mathrm{Ga}$ was directly used for labeling by adding 25 $\mu \mathrm{l}$ of a $1 \mathrm{mg} / \mathrm{ml}$ compound $\mathbf{6 5}$ stock solution in DMSO, followed by $80 \mu 1$ of a $3 \mathrm{~N} \mathrm{NaOAc}$ solution for buffering $(\mathrm{pH}=4-5)$. The mixture was then placed in a thermomixer (FisherSci, USA) at $95^{\circ} \mathrm{C}$ and $750 \mathrm{rpm}$ shaking for 15 minutes. A sample of the reaction was taken and analyzed by radio HPLC to confirm the labeling. Immediately after labeling confirmation, a purification/reformulation was performed by trapping the labeled product in a C-18 Sep-Pak Plus Lite (Waters, USA) followed by a wash with $5 \mathrm{ml}$ of saline solution. The product was then eluted with $200 \mu \mathrm{l}$ of a $50 \% \mathrm{EtOH} / \mathrm{saline}$ solution, followed by 0.9 $\mathrm{ml}$ of saline to produce a final injectable solution containing 8-9\% EtOH in $1 \mathrm{ml}$ saline. A final QC HPLC was performed to assess purity.

Quality Control: Radiochemical and chemical purity were determined on a dual pump Varian Dynamax HPLC (Agilent Technologies, USA) fitted with an Agilent ProStar 325 Dual Wavelength UV-Vis Detector and a NaI(Tl) flow count detector (Bioscan, USA). UV absorption was monitored at $220 \mathrm{~nm}$ and $280 \mathrm{~nm}$. Solvent A was $0.01 \%$ trifluoroacetic acid (TFA) in $\mathrm{H} 2 \mathrm{O}$ and solvent B was $0.01 \%$ TFA in 90:10 v/v MeCN:H2O. Analytical (radio)HPLC was performed a Symmetry® C18, 4.6x50 mm column (Waters, USA), a 
flow rate of $2 \mathrm{~mL} / \mathrm{min}$ and a gradient of 0\% B 0-1 min., 0-100\% B 1-8 min and 100-0\% B 8-10 min. Compound identity was confirmed by co-injection with known standards.

Imaging: Animals (BALB/c) were injected with $100 \mu 1$ of the final solution and placed immediately in the camera under $2 \%$ isoflurane anesthesia. Imaging was performed in a Siemens Inveon $\mu \mathrm{PET} / \mathrm{CT}$ for $30 \mathrm{~min}$ from 45 to 75 minutes post injection. Final Maximum Intensity Projection (MIP/ $\mu$ PET CT) images are presented for 4 animals.

\subsection{Synthesis of adenosine 5'-diphosphate prodrug (AdeDiPPro)}

\section{6-Amino-2-chloro-9-(2'-deoxy-2'-fluoro-3',5'-di- $O$-benzoyl- $\beta$-D-arabino}

furanosyl)-9H-purine (80). Commercially available 1,3,5-tri-O-benzoyl-2-deoxy-2fluoro- $\alpha$-D-arabinofuranose 78 (200 $\mathrm{mg}, 0.43 \mathrm{mmol})$ was placed in a flame-dried flask under $\mathrm{N}_{2}$ atmosphere and treated with $\mathrm{HBr}\left(33 \%\right.$ in acetic acid, $1 \mathrm{~mL}$ ) in $5 \mathrm{~mL}$ of $\mathrm{CH}_{2} \mathrm{Cl}_{2}$ for $12 \mathrm{~h}$. The resulting mixture was neutralized using a concentrated $\mathrm{NaHCO}_{3}$ solution, followed by evaporation of the organic layer to give pure alpha $\mathrm{C} 1$ ' brominated sugar $\mathbf{7 9}^{216}$ (99\%, $180 \mathrm{mg})$. Next, 2-chloroadenine $(71.2 \mathrm{mg}, 0.42 \mathrm{mmol})$ was placed in a flame-dried flask under $\mathrm{N}_{2}$ atmosphere and dissolved with anhydrous $\mathrm{MeCN}$ followed by addition of $\mathrm{KtOBu}$ (catalytic) and $\mathrm{CaH}_{2}(17.4 \mathrm{mg}, 0.42 \mathrm{mmol})$. The suspension was stirred for 40 minutes at $50^{\circ} \mathrm{C}$, then 79 (generated in situ, $180 \mathrm{mg}, 0.42 \mathrm{mmol}$ ) was added and stirring was continued. After $24 \mathrm{~h}$, the residue was filtered with celite, solvents were evaporated, and immediately column chromatographed $(65 \% \mathrm{EtOAc} / \mathrm{Hex})$ to give pure protected $\beta$ clofarabine $\mathbf{8 0}^{216}(55 \%, 118 \mathrm{mg})$ as a solid: ${ }^{1} \mathrm{H} \mathrm{NMR}\left(\mathrm{CDCl}_{3}\right) \delta 4.53-4.58(\mathrm{~m}, 1 \mathrm{H}, \mathrm{H} 4)$, 4.79-4.83 (m, 2H, H5', H5"), 5.85 (dd, $J=2.6,49.8 \mathrm{~Hz}, 1 \mathrm{H}, \mathrm{H} 2$ '), 5.75 (dd, $J=2.8,17.1$ Hz, 1H, H3'), 6.17 (br s, 2H, NH 2 ), 6.57 (dd, $J=2.7,22.6 \mathrm{~Hz}, 1 \mathrm{H}, \mathrm{H1}$ '), 7.42-7.68 (m, 6H, Ar), 8.05-8.13 (m, 5H, H8, Ar). ${ }^{19} \mathrm{~F}$ NMR- H decoupled $\left(\mathrm{CDCl}_{3}\right) \delta$-197.7 (s). 
Note: The 6-Amino-2-chloro-9-(2'-deoxy-2'-fluoro-3',5'-di-O-benzoyl- $\alpha$-D-arabino furanosyl)-9H-purine $\mathbf{8 0 a}^{216}$ was also isolated (55\% EtOAc/Hex) in $40 \%$ yield: ${ }^{1} \mathrm{H}$ NMR $\left(\mathrm{CDCl}_{3}\right) \delta$ 4.71-7.78 (m, 2H, H5', H5"), 4.98 (dd, $J=8.7,4.4 \mathrm{~Hz}, \mathrm{H} 4$ '), 5.82 (dd, $J=17.8$, $1.8 \mathrm{~Hz}, \mathrm{H} 3$ '), 6.00 (s, 2H, 6-NH2), $6.21\left(\mathrm{~d}, J=48.9 \mathrm{~Hz}, \mathrm{H} 2\right.$ '), 6.46 (d, $\left.J=14.3 \mathrm{~Hz}, \mathrm{H} 1{ }^{\prime}\right)$, $7.43(\mathrm{t}, J=7.8 \mathrm{~Hz}, 2 \mathrm{H}, \mathrm{Ar}), 7.48$ (t, $J=7.8 \mathrm{~Hz}, 2 \mathrm{H}, \mathrm{Ar}), 7.60$ (q, $J=7.5 \mathrm{~Hz}, 2 \mathrm{H}, \mathrm{Ar}), 7.80$ (d, $J=7.3 \mathrm{~Hz}, 2 \mathrm{H}, \mathrm{Ar}$ ), 8.04 (s, 1H, H8), 8.12 (d, $J=7.6,6.6 \mathrm{~Hz}, 2 \mathrm{H}, \mathrm{Ar}) .{ }^{19} \mathrm{~F}$ NMR- H decoupled $\left(\mathrm{CDCl}_{3}\right) \delta-187.7(\mathrm{~s})$.

Clofarabine (77). Dibenzoylated clofarabine 80 (50 mg, $0.098 \mathrm{mmol})$ was dissolved in $\mathrm{MeOH}(3 \mathrm{~mL})$ followed by addition of $30 \% \mathrm{w} / \mathrm{w} \mathrm{NaOMe} / \mathrm{MeOH}(1 \mathrm{~mL})$ and stirred at $30^{\circ} \mathrm{C}$ for 30 minutes. Then, solvent was evaporated, and the reaction was immediately column chromatographed $\left(10 \% \mathrm{MeOH} / \mathrm{CH}_{2} \mathrm{Cl}_{2}\right)$ to give $\mathbf{7 7}^{216}(95 \%, 28.6 \mathrm{mg})$ as a solid: ${ }^{1} \mathrm{H}$ NMR (DMSO- $\left.d_{6}\right) \delta 3.60-3.71(\mathrm{~m}, 2 \mathrm{H}, \mathrm{H} 5$ ', H5"), 3.81-3.87 (m, 1H, H4'), 4.42 (dt, $J=$ 4.6, 19.0 Hz, 1H, H3'), 5.23 (dt, $\left.J=4.4,52.6 \mathrm{~Hz}, 1 \mathrm{H}, \mathrm{H2} 2^{\prime}\right), 6.33$ (dd, $J=4.6,13.7 \mathrm{~Hz}, 1 \mathrm{H}$, H1'), 7.88 (br s, 2H, NH$H_{2}$ ), 8.27 (d, $J=2.0 \mathrm{~Hz}, 1 \mathrm{H}, \mathrm{H8}$ ); ${ }^{13} \mathrm{C}$ NMR (DMSO- $d_{6}$ ) 60.7 (C5'), $72.5\left(\mathrm{~d}, J_{\mathrm{C}-\mathrm{F}}=23.2 \mathrm{~Hz}, \mathrm{C}^{\prime}\right), 81.1\left(\mathrm{~d}, J_{\mathrm{C}-\mathrm{F}}=17.0 \mathrm{~Hz}, \mathrm{C}^{\prime}\right), 83.5\left(\mathrm{~d}, J_{\mathrm{C}-\mathrm{F}}=5.6 \mathrm{~Hz}, \mathrm{C} 4^{\prime}\right)$, $94.8\left(\mathrm{~d}, J_{\mathrm{C}-\mathrm{F}}=192.2 \mathrm{~Hz}, \mathrm{C} 2\right.$ '), $117.4(\mathrm{C} 5), 140.1(\mathrm{C} 8), 150.2(\mathrm{C} 6), 153.3(\mathrm{C} 4), 156.9(\mathrm{C} 2)$; ${ }^{19}$ F NMR- H decoupled (DMSO- $d_{6}$ ) $\delta$-198.1 (s).

Clofarabine 5'-monophosphate (81). Clofarabine 77 (26 mg, $0.086 \mathrm{mmol})$ was dissolved in $\mathrm{PO}(\mathrm{OMe})_{3}(1 \mathrm{~mL})$ and cooled to $0^{\circ} \mathrm{C}$ in an ice bath. After this fully dissolved, $\mathrm{POCl}_{3}(24 \mu \mathrm{L}, 39.6 \mathrm{mg}, 0.258 \mathrm{mmol})$ was added and the reaction was stirred at $0{ }^{\circ} \mathrm{C}$ for roughly six hours, when TLC using Todd's system showed full conversion. The reaction was quenched using 0.2M TEAB (triethylammonium bicarbonate) buffer, followed by washing with EtOAc to remove the high boiling point solvent $\left[\mathrm{PO}(\mathrm{OMe})_{3}\right]$. The aqueous 
layer was then was then passed through Sephadex column for initial purification ( 0 to $0.5 \mathrm{M}$ TEAB buffer in $\mathrm{H}_{2} \mathrm{O}$ ), followed by HPLC [A: 20mM TEAA in (1:1) ACN/ $\mathrm{H}_{2} \mathrm{O}$, B: 20mM TEAA in $\mathrm{H}_{2} \mathrm{O}$; System $\rightarrow 0-25 \%$ A in 10 mins, $25 \%$ A for 30 mins; r.t. $=35$ min] to yield pure 81 in 35\% yield (21.2 mg): ${ }^{1} \mathrm{H}$ NMR $\left(\mathrm{CD}_{3} \mathrm{OD}\right) \delta$ 4.12-4.20 (m, 3H, H4', H5', H5"), 4.56-4.64 (m, 1H, H3'), 5.15 (dt, $J=3.5,52.1 \mathrm{~Hz}, 1 \mathrm{H}, \mathrm{H} 2$ '), 6.43 (dd, $J=4.1,15.7 \mathrm{~Hz}, 1 \mathrm{H}$, H1'), 8.34 (s, 1H, H8); ${ }^{19} \mathrm{~F}$ NMR- H decoupled $\left(\mathrm{CD}_{3} \mathrm{OD}\right) \delta-199.7$ (s, 1F); ${ }^{31} \mathrm{P}$ NMR- H decoupled $\left(\mathrm{CD}_{3} \mathrm{OD}\right) \delta 1.01(\mathrm{~s}, 1 \mathrm{P})$

Bis(4-benzoyloxybenzyl)- $N, N$-diisopropylaminophosphoramidites (84). Phenyl benzoate $83(232.7 \mathrm{mg}, 1.174 \mathrm{mmol})$ and freshly distilled $\mathrm{Et}_{3} \mathrm{~N}(180 \mu \mathrm{L}, 130.6 \mathrm{mg}, 1.291$ mmol) were dissolved in THF $(2 \mathrm{~mL})$ in a flame dried flask under $\mathrm{N}_{2}$ atmosphere at $-20^{\circ} \mathrm{C}$. In a separate flame dried flask under $\mathrm{N}_{2}$, commercially available dichloro$\mathrm{N}, \mathrm{N}$,diisopropylaminophosphoramidite $(130 \mu \mathrm{L}, 118.7 \mathrm{mg}, 0.587 \mathrm{mmol})$ was dissolved in $2 \mathrm{~mL}$ THF at $-20^{\circ} \mathrm{C}$. Then, the phenyl benzoate solution was added dropwise to the dichloro phosphoramidite reagent with formation of a precipitate and gas. The mixture was stirred at $-20^{\circ} \mathrm{C}$ for 30 minutes, when TLC $(10 \%$ EtOAc/Hex +3 drops TEA) confirmed formation of product. The solvent was then evaporated and the crude reaction mixture was used for synthesis of DiPPro 87 . NMR showed roughly $85 \%$ conversion to $\mathbf{8 4}^{52}$ with decomposition of roughly $30 \%$ per day of storage: ${ }^{1} \mathrm{H}$ NMR $\left(\mathrm{CDCl}_{3}\right) \delta 1.25-136\left(\mathrm{~m}, 12 \mathrm{H}, 4-\mathrm{CH}_{3}\right), 3.66-$ $3.72(\mathrm{~m}, 2 \mathrm{H}, 2-\mathrm{CH}), 4.70-4.83\left(\mathrm{~m}, 4 \mathrm{H}, 2-\mathrm{CH}_{2}\right), 7.17-7.20(\mathrm{~m}, 4 \mathrm{H}, \mathrm{Ar}), 7.40-7.45(\mathrm{~m}, 4 \mathrm{H}$, Ar), 7.49-7.53 (m, 4H, Ar), 7.61-7.66 (m, 2H, Ar), 8.18-8.23 (m, 4H, Ar); ${ }^{31}$ P NMR- H decoupled $\left(\mathrm{CDCl}_{3}\right) \delta 147.94(\mathrm{~s}, 1 \mathrm{P})$

Adenosine bis(benzoyloxybenzyl)-5'-diphosphate DiPPro (87). Phosphoramidite 84 ( 200mg, generated in situ in excess) was stirred in a solution containing 5-phenyl-1- 
$H$-tetrazole activator $(0.309 \mathrm{mmol}, 45 \mathrm{mg})$ in $5 \mathrm{~mL}$ DMF. Then, after 10 minutes, this mixture was added to a solution of adenosine 5'-monophosphate triethylammonium salt 86 (0.103 mmol, $60 \mathrm{mg})$ in $5 \mathrm{~mL}$ DMF. After $1 \mathrm{~h}, \mathrm{tBuOOH}(5 \mathrm{eq}, 0.515 \mathrm{mmol}, 46.4 \mathrm{mg}, 50$ $\mu \mathrm{L}$ ) was added to oxidize and stirred for an additional hour. Solvents were evaporated under vacuum, and compound $\mathbf{8 7}$ was separated by crystallization using ethyl acetate: ${ }^{1} \mathrm{H}$ NMR $\delta$ 3.4-3.52 (m, 4H, 2CH 2 ), 4.20-4.34 (m, 2H, H5', H5"), 4.39-4.42 (m, 1H, H4'), 4.56$4.61\left(\mathrm{~m}, 1 \mathrm{H}, \mathrm{H} 3^{\prime}\right), 5.12-5.21\left(\mathrm{~m}, 1 \mathrm{H}, \mathrm{H} 2^{\prime}\right), 6.11\left(\mathrm{~d}, J=5.7 \mathrm{~Hz} 1 \mathrm{H}, \mathrm{H} 1^{\prime}\right), 7.19-7.23(\mathrm{~m}, 4 \mathrm{H}$, Ar), 7.43-7.47 (m, 4H, Ar), 7.53-7.58 (m, 4H, Ar), 7.67-7.72 (m, 2H, Ar), 8.14-8.18 (m, 4H, Ar), 8.22 (s, 1H, H8), 8.53 (s, 1H, H2); ${ }^{13} \mathrm{C}$ NMR $\delta$ 19.43, 70.6, 72.1, 76.2, 85.5, 91.1, $121.5,123.0,129.8,130.5,130.7,131.1,134.9,135.0,141.2,149.8,152.3,152.8,156.5$ 166.4; ${ }^{31} \mathrm{P}$ NMR $\delta-12.07\left(\mathrm{~d}, J=19.8 \mathrm{~Hz}, \mathrm{P}_{\alpha}\right),-12.71\left(\mathrm{~d}, J=19.8 \mathrm{~Hz}, \mathrm{P}_{\beta}\right) ;$ LRMS (ESI+) $m / z$ calcd for $\mathrm{C}_{38} \mathrm{H}_{34} \mathrm{~N}_{5} \mathrm{O}_{14} \mathrm{P}_{2}[\mathrm{M}-\mathrm{H}]^{-}, 846.1583$; found 846.2.

\subsection{Synthesis of azido nucleoside analogues}

\subsubsection{2-Azido purine analogues}

\section{2-Azido-6-chloro-9-(3,5-di-O-acetyl-2-deoxy- $\beta$-D-erythro-pentofuranosyl)purine}

(96). Compound $\mathbf{9 5}^{237}(0.121 \mathrm{mmol}, 45 \mathrm{mg})$ was dried for several hours under vacuum, and then placed in a flame dried flask under $\mathrm{N}_{2}$ atmosphere. Then, it was dissolved in 5 $\mathrm{mL}$ of $\mathrm{MeCN}$ and cooled to $-20^{\circ} \mathrm{C}$ in a salt ice bath. To this mixture, $\mathrm{tBuONO}(5 \mathrm{eq}, 0.605$ mmol, $62.4 \mathrm{mg}, 72 \mu \mathrm{L})$ was added and stirred for five minutes. Then, TMS-N 3 ( 5 eq, 0.605 mmol, $69.7 \mathrm{mg}, 80 \mu \mathrm{L}$ ) was added, and the mixture was allowed to stand in a $-20^{\circ} \mathrm{C}$ freezer. After 12 hours, the reaction was placed in a $0^{\circ} \mathrm{C}$ refrigerator for an additional 24 hours. Solvents were evaporated under vacuum, and crude mixture was column chromatographed using a hexane/ethyl acetate gradient $\left(10 \% \rightarrow 70 \%\right.$ EtOAc) to give $96(63 \%, 30.1 \mathrm{mg}):{ }^{1} \mathrm{H}$ 
$\operatorname{NMR}\left(\mathrm{CDCl}_{3}\right) \delta 2.09\left(\mathrm{~s}, 3 \mathrm{H}, \mathrm{CH}_{3}\right), 2.14\left(\mathrm{~s}, 3 \mathrm{H}, \mathrm{CH}_{3}\right), 2.60-2.67$ (m, 1H, H2'), 2.71-2.79 (m, 1H, H2"), 4.30-4.41 (m, 3H, H4', H5', H5"), 5.38-5.41 (m, 1H, H3'), 6.41 (dd, J = 6.1, $\left.7.7 \mathrm{~Hz}, 1 \mathrm{H}, \mathrm{H} 1^{\prime}\right), 8.21$ (s, 1H, H8).

2-Azido-2'-deoxyadenosine (90). Compound 96 (0.076 mmol, $30 \mathrm{mg})$ was dried under vacuum and placed in an ice salt bath to cool. Then, it was dissolved in $5 \mathrm{~mL}$ of cold $\mathrm{EtOH} / \mathrm{NH}_{3}$ saturated solution, sealed tightly, and placed in a $30^{\circ} \mathrm{C}$ oil bath to stir for 2 days. The reaction was then cooled, and pressure was released to evaporate solvent under vacuum. Then, fresh $\mathrm{EtOH} / \mathrm{NH}_{3}$ reagent was added, the flask was sealed tightly, and stirring at $30^{\circ} \mathrm{C}$ was continued. After an additional 2 days, the solvent was evaporated and the crude mixture was column chromatographed using $\mathrm{CHCl}_{3} / \mathrm{MeOH}$ gradient $(0 \% \rightarrow 10 \%$ $\mathrm{MeOH})$. Further purification by HPLC was performed using $\mathrm{H}_{2} \mathrm{O} / \mathrm{MeCN}$ system $(0 \% \rightarrow$ $20 \% \mathrm{MeCN}$ gradient over $30 \mathrm{mins}$, then $20 \% \mathrm{MeCN}$ from $30-40 \mathrm{mins}$ ), to give $\mathbf{9 0}^{142}$ (80\%, $17.8 \mathrm{mg}):{ }^{1} \mathrm{H}$ NMR $\left(\mathrm{CD}_{3} \mathrm{OD}\right) \delta$ 2.32-2.40 (m, 1H, H2'), 2.71-2.79 (m, 1H, H2"), 3.63 (dd, $\left.J=4.1,12.2 \mathrm{~Hz}, 1 \mathrm{H}, \mathrm{H}^{\prime}\right), 3.74\left(\mathrm{dd}, J=3.3,12.2 \mathrm{~Hz}, 1 \mathrm{H}, \mathrm{H} 5^{\prime}\right), 4.02-4.08$ (m, 1H, H4'), 4.53-4.58 (m, 1H, H3'), 6.32-6.39 (dd, $\left.J=6.3,7.5 \mathrm{~Hz}, 1 \mathrm{H}, \mathrm{H} 1^{\prime}\right), 8.20$ (s, 1H, H8).

Note: Compound 90 is in equilibrium with corresponding tetrazole derivative (90a, $13.5 \%)$ as judged by the analysis of proton NMR spectra: ${ }^{1} \mathrm{H}$ NMR $\left(\mathrm{CD}_{3} \mathrm{OD}\right) \delta 6.47-6.52$ (m, H1'), $8.48(\mathrm{~s}, \mathrm{H} 8)$.

2-Azido-2' -deoxyinosine (89). Compound 90 (0.017 mmol, $5 \mathrm{mg}$ ) was dissolved in 2 $\mathrm{mL}$ 0.05 $\mathrm{M}$ phosphate buffer $\left(\mathrm{K}_{3} \mathrm{PO}_{4}, \mathrm{pH}=7.4\right)$, and stirred at $0^{\circ} \mathrm{C}$ until dissolved. To this, $1 \mathrm{mg}$ ( 22.1 units as specified by manufacturer) of adenosine deaminase enzyme was added and stirred at $30^{\circ} \mathrm{C}$ for $24 \mathrm{~h}$. Conversion was determined by TLC with formation of fluorescence spot. To achieve full conversion, an additional $2 \mathrm{mg}$ ( 44.2 units) of 
adenosine deaminase was added to the reaction. After stirring for 7 days total, the reaction was filtered and solution was purified with reverse phase $\mathrm{HPLC}\left(\mathrm{H}_{2} \mathrm{O} / \mathrm{MeCN}, 0 \% \rightarrow 15 \%\right.$ $\mathrm{MeCN}$ in $30 \mathrm{mins})$ to give $\mathbf{8 9}^{222}(4 \mathrm{mg}, 81 \%):{ }^{1} \mathrm{H} \mathrm{NMR}\left(\mathrm{CD}_{3} \mathrm{OD}\right) \delta 2.46-2.53(\mathrm{~m}, 1 \mathrm{H}$, H2'), 2.85-2.92 (m, 1H, H2"), 3.79 (dd, $J=3.7,12.2 \mathrm{~Hz}, 1 \mathrm{H}, \mathrm{H} 5$ '), 3.92 (dd, $J=3.2,12.2$ Hz,1H, H5'), 4.09-4.13 (m, 1H, H4'), 4.63-4.69 (m, 1H, H3'), 6.48 (dd, J = 6.2, 7.6 Hz, 1H, H1'), 8.22 (s, 1H, H8).

2-Azidoadenosine (92). Protected 2-azido-6-chloro purine 100 $^{146,211}$ (0.088 mmol, 40 $\mathrm{mg}$ ) was dried under vacuum, cooled in an ice salt bath, and $7 \mathrm{~mL} \mathrm{EtOH} / \mathrm{NH}_{3}$ reagent was added. Then, the flask was sealed tightly, and placed in a $30^{\circ} \mathrm{C}$ oil bath to stir for 2 days, with addition of more $\mathrm{EtOH} / \mathrm{NH}_{3}$ if based on TLC analysis of product formation. After an additional 2 days, the solvent was evaporated, and the crude mixture was column chromatographed using $\mathrm{CHCl}_{3} / \mathrm{MeOH}$ gradient $(0 \% \rightarrow 10 \% \mathrm{MeOH})$, followed by purification on reverse phase $\mathrm{HPLC}$ with $\mathrm{H}_{2} \mathrm{O} / \mathrm{MeCN}$ gradient $(0 \% \rightarrow 20 \% \mathrm{MeCN}$ gradient over 30 mins, then $20 \% \mathrm{MeCN}$ from 30-40 mins) to give $\mathbf{9 2}^{146}$ (70\%, $19 \mathrm{mg}$ ): ${ }^{1} \mathrm{H}$ NMR $\left(\mathrm{CD}_{3} \mathrm{OD}\right) \delta 3.75\left(\mathrm{dd}, J=3.2,12.4 \mathrm{~Hz}, 1 \mathrm{H}, \mathrm{H} 5{ }^{\prime}\right), 3.87$ (dd, $\left.J=2.8,12.4 \mathrm{~Hz}, 1 \mathrm{H}, \mathrm{H} 5 "\right), 4.12-$ 4.15 (m, 1H, H4'), 4.28-4.33 (m, 1H, H3'), 4.65-4.71 (m, 1H, H2'), 5.80 (d, J= 6.1 Hz, 1H, H1'), 8.25 (s, 1H, H8).

Note: Compound 92 is in equilibrium with corresponding tetrazole derivative (92a, $9.0 \%)$ as judged by the analysis of proton NMR spectra: ${ }^{1} \mathrm{H}$ NMR $\left(\mathrm{CD}_{3} \mathrm{OD}\right) \delta 6.08(\mathrm{~d}, J=$ $5.4 \mathrm{~Hz}, \mathrm{H1}$ ), 8.49 (s, 1H, H8).

2-Azidoinosine (91). Compound $92(0.081 \mathrm{mmol}, 25 \mathrm{mg})$ was dissolved in $10 \mathrm{~mL} 0.05$ $\mathrm{M}$ phosphate buffer $\left(\mathrm{K}_{3} \mathrm{PO}_{4}, \mathrm{pH}=7.4\right)$, and stirred at $0^{\circ} \mathrm{C}$ until dissolved. To this, $3 \mathrm{mg}(\sim$ 66.3 units as specified by manufacturer) of adenosine deaminase enzyme was added and 
stirred at $30^{\circ} \mathrm{C}$ for 1 day. To achieve full conversion, as seen by formation of a fluorescence spot on TLC, an additional $2 \mathrm{mg}$ ( 44.2 units) of adenosine deaminase was added to the reaction. After stirring for 3 days total, the reaction was filtered and solution was injected directly onto HPLC. A gradient of water/MeCN $(0 \% \rightarrow 10 \% \mathrm{MeCN}$ in 40 mins) was used to elute compound $91(20 \mathrm{mg}, 80 \%) .{ }^{1} \mathrm{H}$ NMR $\left(\mathrm{CD}_{3} \mathrm{OD}\right) \delta 3.77(\mathrm{dd}, J=3.0,12.5 \mathrm{~Hz}, 1 \mathrm{H}$, H5'), 3.92 (dd, $J=2.6,12.5 \mathrm{~Hz}, 1 \mathrm{H}, \mathrm{H} 5 "), 4.14-4.18$ (m, 1H, H4'), 4.32-4.38 (m, 1H, H3'), $4.61(\mathrm{~b}, 1 \mathrm{H}, \mathrm{NH}), 4.78(\mathrm{t}, J=5.5 \mathrm{~Hz}, 1 \mathrm{H}, \mathrm{H} 2$ '), $5.98(\mathrm{~d}, J=5.9 \mathrm{~Hz}, 1 \mathrm{H}, \mathrm{H1}$ '), $8.15(\mathrm{~s}, 1 \mathrm{H}$, $\mathrm{H} 8)$.

\subsubsection{6-Azido purine analogues}

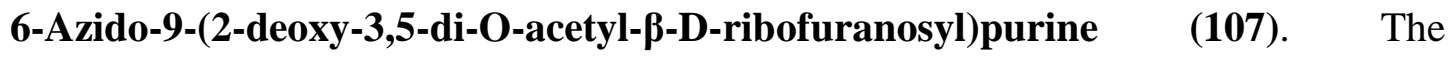
6-chloropurine 104 (36 mg, $0.10 \mathrm{mmol})$ and $\mathrm{NaN}_{3}(65 \mathrm{mg}, 1 \mathrm{mmol})$ were dissolved in DMF $(5 \mathrm{~mL})$ and placed in at $80^{\circ} \mathrm{C}$ with stirring for 3 hours. Then, DMF was separated from the organic products by separation with EtOAc and water, followed by evaporation of the organic layer. The resulting residue was column chromatographed (85\% EtOAC/Hex) to give $107^{140}(55 \%, 19.1 \mathrm{mg}$ ) (as a mixture of azido/tetrazole tautomers in a nearly 1:1 ratio).

The azido tautomer had: ${ }^{1} \mathrm{H}$ NMR $\left(\mathrm{CDCl}_{3}\right) \delta 2.10\left(\mathrm{~s}, 3 \mathrm{H}, \mathrm{CH}_{3}\right), 2.12\left(\mathrm{~s}, 3 \mathrm{H}, \mathrm{CH}_{3}\right), 2.64-$ 2.72 (m, 1H, H2'), 2.92-2.99 (m, 1H, H2"), 4.38-4.45 (m, 3H, H4', H5', H5"), 5.46-5.52 (m, 1H, H3'), 6.51 (dd, $J=6.1,7.8 \mathrm{~Hz}, 1 \mathrm{H}, \mathrm{H} 1$ '), 8.21 (s, 1H, H8), 8.43 (s, 1H, H2).

Note: Compound 107 was found to be in equilibrium with its tetrazole tautomer (107a, $76 \%)$ as judged by analysis of proton NMR spectra: ${ }^{1} \mathrm{H} \mathrm{NMR}\left(\mathrm{CDCl}_{3}\right) \delta 2.17\left(\mathrm{~s}, 3 \mathrm{H}, \mathrm{CH}_{3}\right)$, 2.19 (s, 3H, $\mathrm{CH}_{3}$ ), 2.77-2.83 (m, 1H, H2'), 3.00-3.05 (m, 1H, H2"), 4.46-4.49 (m, 3H, H4', H5', H5"), 5.53-5.56 (m, 1H, H3'), 6.63 (dd, $J=6.2,7.6 \mathrm{~Hz}, 1 \mathrm{H}, \mathrm{H1}$ '), 8.71 (s, 1H, H8), $9.55(\mathrm{~s}, 1 \mathrm{H}, \mathrm{H} 2)$. 


\subsubsection{4-Azido pyrimidine analogues}

1-(3,5-Tri-O-acetyl- $\beta$-D-deoxyribofuranose)-4-azido-2-pyrimidinone (112). The 4-

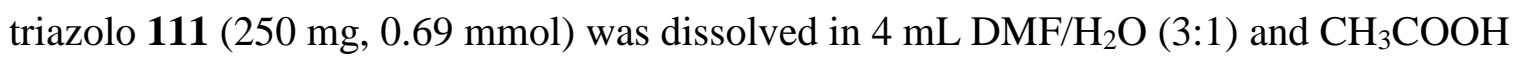
$(500 \mu \mathrm{L}, 525 \mathrm{mg}, 8.74 \mathrm{mmol})$ was added. The $\mathrm{NaN}_{3}(280.4 \mathrm{mg}, 4.31 \mathrm{mmol})$ was dissolved in $\mathrm{H}_{2} \mathrm{O}(1 \mathrm{~mL})$ and added to the reaction. The mixture was stirred at $50^{\circ} \mathrm{C}$ for roughly 72 h. Then, the solution was diluted with $20 \mathrm{~mL}$ EtOAc, and washed with $\mathrm{NaHCO}_{3}, \mathrm{H}_{2} \mathrm{O}$, and brine. The organic layer was evaporated and column chromatographed (80\% EtOAc/Hex) to give $112(85 \%, 198 \mathrm{mg}):{ }^{1} \mathrm{H}$ NMR $\left(\mathrm{CDCl}_{3}\right) \delta 2.11\left(\mathrm{~s}, 3 \mathrm{H}, \mathrm{CH}_{3}\right), 2.16\left(\mathrm{~s}, 3 \mathrm{H}, \mathrm{CH}_{3}\right), 2.22-$ 2.31 (m, 1H, H2'), 2.72-2.80 (m, 1H, H2"), 4.33-4.41 (m, 2H, H4', H5'), 4.45 (dd, J = 3.4, 11.4 Hz, 1H, H5"), 5.27-5.31 (m, 1H, H3'), 6.52 (dd, J = 5.6, 8.1 Hz, 1H, H1'), 6.95 (d, J= $7.9 \mathrm{~Hz}, 1 \mathrm{H}, \mathrm{H} 5), 7.77$ (d, $J=7.9 \mathrm{~Hz}, 1 \mathrm{H}, \mathrm{H} 6)$.

6-(2-Deoxy- $\beta$-D-ribosyl)tetrazolo[1,5-c $]$ pyrimidin-5(6H)-one (113). Compound 112 (50 mg, $0.15 \mathrm{mmol}$ ) was dissolved in $2 \mathrm{~mL}$ of $\mathrm{MeOH}$ and cooled to $0^{\circ} \mathrm{C}$, then roughly 2 $\mathrm{mL}$ of $\mathrm{MeOH}$ saturated with $\mathrm{HCl}$ was added. The solution was stirred for $3 \mathrm{~h}$ at $0^{\circ} \mathrm{C}$. The solvent was evaporated, and the residue was immediately column chromatographed (5\% $\left.\mathrm{MeOH} / \mathrm{CH}_{2} \mathrm{Cl}_{2}\right)$ to give $113(95 \%, 36.1 \mathrm{mg}):{ }^{1} \mathrm{H} \mathrm{NMR}\left(\mathrm{CD}_{3} \mathrm{OD}\right) \delta 2.43-2.48(\mathrm{~m}, 1 \mathrm{H}, \mathrm{H} 2$ '), 2.52-2.55 (m, 1H, H2"), 3.72 (dd, $J=3.5,12.1 \mathrm{~Hz}, 1 \mathrm{H}, \mathrm{H} 5$ '), 3.87 (dd, $J=3.1,12.1 \mathrm{~Hz}$, 1H, H5"), 4.05-4.10 (m, 1H, H4'), 4.43-4.49 (m, 1H, H3'), 6.52 (t, J = 6.5 Hz, 1H, H1'), $7.02(\mathrm{~d}, J=7.8 \mathrm{~Hz}, 1 \mathrm{H}, \mathrm{H} 5), 8.33(\mathrm{~d}, J=7.9 \mathrm{~Hz}, 1 \mathrm{H}, \mathrm{H} 6)$.

\section{1-(2',3',5'-Tri- $O$-acetyl- $\beta$-D-ribofuranosyl)-4-azidopyrimidin-2(1H)-one}

(114).

Compound 118 (150 mg, $0.36 \mathrm{mmol}$ ) was dissolved in $4 \mathrm{~mL} \mathrm{DMF} / \mathrm{H}_{2} \mathrm{O}$ (3:1) and $\mathrm{CH}_{3} \mathrm{COOH}(261 \mu \mathrm{L}, 274 \mathrm{mg}, 4.56 \mathrm{mmol})$ was added. The $\mathrm{NaN}_{3}(146.3 \mathrm{mg}, 2.25 \mathrm{mmol})$ was dissolved in $\mathrm{H}_{2} \mathrm{O}(1 \mathrm{~mL})$ and added to the reaction. The mixture was stirred at $50^{\circ} \mathrm{C}$ 
for roughly $48 \mathrm{~h}$. Then, the solution was diluted with $20 \mathrm{~mL}$ EtOAc, and washed with $\mathrm{NaHCO}_{3}, \mathrm{H}_{2} \mathrm{O}$, and brine. The organic layer was evaporated and column chromatographed (90\% EtOAc/Hex) to give $114{ }^{238}(75 \%, 107 \mathrm{mg}):{ }^{1} \mathrm{H} \mathrm{NMR}\left(\mathrm{CDCl}_{3}\right) \delta 2.11\left(\mathrm{~s}, 3 \mathrm{H}, \mathrm{CH}_{3}\right)$, 2.21 (s, 3H, $\left.\mathrm{CH}_{3}\right), 4.39-4.43$ (m, 1H, H5'), 4.48-4.55 (m, 2H, H4', H5"), 5.43-5.48 (m, 2H, H2', H3'), 6.37 (d, J = 5.0 Hz, 1H, H1'), 6.98 (d, $J=7.8 \mathrm{~Hz}, 1 \mathrm{H}, \mathrm{H} 5), 7.64$ (d, $J=7.9 \mathrm{~Hz}$, 1H, H6).

6-( $\beta$-D-ribofuranosyl)tetrazolo[1,5-c]pyrimidin-5(6H)-one (115). Compound 114 (85 mg, $0.22 \mathrm{mmol}$ ) was dissolved in $2 \mathrm{~mL}$ of $\mathrm{MeOH}$ and cooled to $0^{\circ} \mathrm{C}$, then roughly 2 $\mathrm{mL}$ of $\mathrm{MeOH}$ saturated with $\mathrm{HCl}$ was added. The solution was stirred for $3 \mathrm{~h}$ at $0^{\circ} \mathrm{C}$. The solvent was evaporated, and the residue was immediately column chromatographed (5\% $\left.\mathrm{MeOH} / \mathrm{CH}_{2} \mathrm{Cl}_{2}\right)$ to give $115(90 \%, 53.2 \mathrm{mg}) .{ }^{1} \mathrm{H} \mathrm{NMR}\left(\mathrm{CD}_{3} \mathrm{OD}\right) \delta 3.83(\mathrm{dd}, J=2.7,12.3$ Hz, 1H, H5'), 3.96 (dd, $J=3.1,12.1 \mathrm{~Hz}, 1 \mathrm{H}, \mathrm{H} 5 "), 4.13-4.16$ (m, 1H, H4'), 4.23-4.27 (m, 1H, H3'), 4.32-4.35 (m, 1H, H2'), 6.18 (d, $\left.J=3.9 \mathrm{~Hz}, 1 \mathrm{H}, \mathrm{H} 1^{\prime}\right), 7.01$ (d, $J=7.9 \mathrm{~Hz}, 1 \mathrm{H}$, H5), 8.39 (d, J = 7.9 Hz, 1H, H6).

\subsection{ESR studies of azido-modified nucleosides}

\subsubsection{Sample preparation and methodology}

Transparent glassy samples of azido-modified nucleosides were prepared. Subsequently, $\gamma$-irradiation and ESR spectral analyses of these samples were performed. Compounds purchased: Lithium chloride ( $\mathrm{LiCl}$ ) (ultra-dry, 99.995\% (metals basis)) was obtained from Alfa Aesar (Ward Hill, MA, USA). Deuterium oxide ( $\left.\mathrm{D}_{2} \mathrm{O}\right)(99.9$ atom \% D) was purchased from Aldrich Chemical Company Inc. (Milwaukee, WI, USA). All compounds were used without further purification. 
Glassy sample preparation: As per the ongoing studies of Sevilla-Adhikary lab at Oakland University, ${ }^{93,94,104-106,227,229}$ transparent homogeneous glassy sample were prepared as follows: (i) Preparation of homogeneous solutions: First, homogeneous solution was prepared by dissolving ca. 1.0 to $2.4 \mathrm{mg} / \mathrm{mL}$ of a compound (e.g., 4) in either 7.5 M LiCl in $\mathrm{D}_{2} \mathrm{O}$. The native $\mathrm{pH}$ of $7.5 \mathrm{M} \mathrm{LiCl}$ in $\mathrm{D}_{2} \mathrm{O}$ is ca. 5 and $\mathrm{pH}$ of these solutions was not adjusted. (ii) Preparation of glassy samples and their storage: Homogenous solutions of azido compounds (as indicated in (i)) were thoroughly bubbled with nitrogen gas to avoid formation of peroxyl radicals. Subsequently, those solutions were immediately drawn into $4 \mathrm{~mm}$ Suprasil quartz tubes (Catalog no. 734-PQ-8, WILMAD Glass Co., Inc., Buena, NJ, USA). Thereafter, the quartz tubes containing these solutions were rapidly immersed in liquid nitrogen $(77 \mathrm{~K})$. Owing to rapid cooling at $77 \mathrm{~K}$, the homogeneous liquid solutions formed transparent homogeneous glassy solutions. Subsequently, these transparent homogeneous glassy solutions were $\gamma$-irradiated at $77 \mathrm{~K}$ and were subjected to progressive annealing experiments or photoexcitation along with ESR spectral studies. All glassy samples were stored in the dark at $77 \mathrm{~K}$ in Teflon containers prior to and after $\gamma$ irradiation.

$\gamma$-Irradiation of glassy samples and their storage: As per well-established methodology of $\gamma$-irradiation of glassy samples of DNA and RNA-models, ${ }^{93-95,209,222,224,225}$ the glassy samples were $\gamma(60 \mathrm{Co})$-irradiated (absorbed dose $=600 \mathrm{~Gy}(2.5 \mathrm{~h})$ ) at $77 \mathrm{~K}$ and stored at $77 \mathrm{~K}$ in Teflon containers in the dark. Following our previous work with 3'-AZT, ${ }^{104}$ azidopentofuranoses, ${ }^{105}$ and other azido-nucleosides, ${ }^{106}$ the solute concentrations were kept low $(2-3 \mathrm{mg} / \mathrm{mL})$ of $7.5 \mathrm{M} \mathrm{LiCl}$ glass $\left(\mathrm{D}_{2} \mathrm{O}\right)$. As a result, $\mathrm{Cl}_{2}{ }^{-}$is formed owing to scavenging 
of radiation-induced holes by the matrix $(7.5 \mathrm{M} \mathrm{LiCl})$ and the radiation-produced electrons in the glass are scavenged by the solute of azido compounds.

Annealing of glassy samples: As per previous studies, ${ }^{93-95,209,222,224,225}$ a variable temperature assembly that passed liquid nitrogen cooled dry nitrogen gas past a thermister and over the glassy sample was employed for annealing. Stepwise (either $5 \mathrm{~K}$ or $10 \mathrm{~K}$ step) annealing of each glassy sample was conducted in the range (140-170) $\mathrm{K}$ for $15 \mathrm{~min}$. The matrix radical, $\mathrm{Cl}_{2} \bullet^{-}$, did not react with sample. Thus, by employing ESR spectroscopy, we were able to study directly the formation of $\mathrm{RNH} \bullet$ via reaction of radiation-produced electron with azido compounds and subsequent reactions of $\mathrm{RNH} \bullet$.

Photoexcitation: Following our works that involve photoexcitation, ${ }^{93,94,227}$ a thermoelectrically cooled blue laser (TECBL-30G-405, World Star Tech., Lot 6880, $\lambda=405$ nm, $30 \mathrm{~mW}$ ), a helical quartz low-pressure argon-mercury vapor lamp (90\% emission at 254 $\mathrm{nm}$ ), and a photoflood lamp were employed for photoexcitation experiments at $77 \mathrm{~K}$.

Electron Spin Resonance: As per ongoing studies in Sevilla-Adhikary laboratory, ${ }^{93-}$ 95,209,222,224,225 we used a Varian E-Century Series X-band (9.3 GHz) ESR spectrometer with an E-4531 dual cavity, 9-inch magnet, and a $200 \mathrm{~mW}$ Klystron. For the field calibration, Fremy's salt $($ gcenter $=2.0056, \mathrm{~A}(\mathrm{~N})=13.09 \mathrm{G})$ was employed. All ESR spectra were recorded at $77 \mathrm{~K}$ and at $45 \mathrm{~dB}(6.3 \mu \mathrm{W})$ as well as $40 \mathrm{~dB}(20 \mu \mathrm{W})$. We note here that recording of ESR spectra at $77 \mathrm{~K}$ maximizes the signal height and allows for comparison of signal intensities.

Employing the Bruker programs (WIN-EPR and SimFonia) and ongoing studies on DNA and RNA-radicals, anisotropic simulations of experimentally recorded ESR spectra were carried out. The ESR parameters (e.g., hyperfine coupling constant (HFCC) values, 
linewidth, etc.) were adjusted to obtain the "best fit" simulated spectrum that matched the experimental ESR spectrum well.

Method of theoretical Calculations: Employing optimized geometries of radicals, energies of radicals and hyperfine coupling constant (HFCC) values were calculated using DFT/B3LYP/6-31+G** method in Gaussian 09. Theoretically predicted HFCC values obtained employing B3LYP/6-31+G** method agree well with those obtained using experiment. Jmol molecular modeling freeware was used to plot optimized molecular structures.

\subsection{In vivo biological evaluation of azido-modified nucleosides}

The MB-MDA-231, MCF7 and U87 cells were seeded in 96 well plate $(10,000$ cells/well). Azido-modified nucleosides were dissolved in DMSO, and $200 \mu \mathrm{M}$ stock solutions of each were prepared. The next day, cells were treated with $100 \mu \mathrm{M}$ of each compound (i.e. compounds $112,113,107$, and 89 ) by diluting them in culture media. After $24 \mathrm{~h}$ of compound treatment, the cells were irradiated with $2 \mathrm{~Gy}$ and $4 \mathrm{~Gy}$ of gamma irradiation in Shepherd Mark I 137Cs irradiator (Model 25, J. L. Shepherd \& Associates, San Fernando, CA, USA). After 24 and $48 \mathrm{~h}$ of irradiation, cell viability was checked with MTS cell proliferation assay (Promega). For the assay, the old media were discarded, and the cells were washed with fresh media. Then, $20 \mu \mathrm{L}$ of MTS and $1 \mu \mathrm{L}$ of PES solution was added with $100 \mu \mathrm{L}$ of fresh media in each well. After $1 \mathrm{~h}$ in incubation, absorbance was measured in BioTeK microplate reader at $490 \mathrm{~nm}$.

\subsection{Synthesis of 3',5'-di-O-acetyl-4-azido-5',5'-dideutero-2'-deoxyuridine}

Methyl 2-Deoxy-5,5'-dideuteroriboside (131). The dry commercially available 2deoxy-5,5'-dideuteroribose $\mathbf{1 3 0}(200 \mathrm{mg}, 1.47 \mathrm{mmol})$ was added to a dry flask and 
dissolved in $5 \mathrm{~mL}$ methanol. The mixture was place in a $0^{\circ} \mathrm{C}$ ice bath, and concentrated $\mathrm{H}_{2} \mathrm{SO}_{4}(20 \mu \mathrm{L}, 36.8 \mathrm{mg}, 0.375 \mathrm{mmol})$ was added. The reaction was stirred for 1.5 hours at $0^{\circ} \mathrm{C}$, followed by neutralization with pyridine $(760 \mu \mathrm{L})$. After evaporation under vacuum, the dry residue was column chromatographed $\left(0 \% \rightarrow 10 \% \mathrm{MeOH} / \mathrm{CH}_{2} \mathrm{Cl}_{2}\right)$ to give $207 \mathrm{mg}$ (94\%) as mixture of isomers. The methyl deoxyribofuranoside derivative $131(45 \%)$ had: ${ }^{1} \mathrm{H} \mathrm{NMR}\left(\mathrm{CDCl}_{3}\right) \delta$ 2.12-2.17 (m, 2H, H2, H2' pyranose), 2.32 (ddd, $J=2.1,6.9,14.0 \mathrm{~Hz}$, $1 \mathrm{H}, \mathrm{H} 2$ '), 3.45 (s, 3H, $\left.\mathrm{CH}_{3}\right), 4.09-4.12(\mathrm{~m}, 1 \mathrm{H}, \mathrm{H} 3), 4.56-4.59(\mathrm{~m}, 1 \mathrm{H}, \mathrm{H} 4), 5.17$ (dd, $J=$ 2.0, $5.7 \mathrm{~Hz}, 1 \mathrm{H}, \mathrm{H1})$.

The methyl deoxyribopyranoside derivative (55\%) had: ${ }^{1} \mathrm{H} \mathrm{NMR}\left(\mathrm{CDCl}_{3}\right) \delta$ 1.87-1.93 (m, 1H, H2), 2.12-2.17 (m, H, H2', H2 furanose), 3.42 (s, 3H, $\left.\mathrm{CH}_{3}\right)$, 4.16-4.19 (m, 2H, H3, $\mathrm{H} 4), 5.14(\mathrm{~d}, J=4.5 \mathrm{~Hz}, 1 \mathrm{H}, \mathrm{H} 1)$.

Methyl 3,5-Di- $\boldsymbol{O}$-acetyl-2-deoxy-5,5'-dideuteroriboside (132). Pure 131 (207 mg, $1.38 \mathrm{mmol}$ ) was dissolved in $4 \mathrm{~mL}$ of pyridine and cooled to $0^{\circ} \mathrm{C}$ on an ice bath. Slowly, 2 $\mathrm{mL}$ of $\mathrm{Ac}_{2} \mathrm{O}$ was added, and the mixture was allowed to warm to room temperature. This was stirred for 2 days, and upon reaction completion based on TLC analysis, the solvents were evaporated under vacuum. The residue was dissolved in $\mathrm{CH}_{2} \mathrm{Cl}_{2}$ and washed with dilute $\mathrm{HCl}, \mathrm{NaHCO}_{3}$, and brine. Then, the mixture was column chromatographed $(0 \% \rightarrow$ $50 \%$ EtOAc/Hexane) to give $258 \mathrm{mg}(80 \%)$ as a mixture of isomers. The deoxyribofuranoside derivative (132, 38\%) had: ${ }^{1} \mathrm{H} \mathrm{NMR}\left(\mathrm{CDCl}_{3}\right) \delta 2.11$ (s, 3H, Ac), 2.12 (s, 3H, Ac), 2.37-2.45 (m, 2H, H2, H2'), 3.35 (s, 3H, CH 3 ), 4.23 (d, J= 3.0 Hz, 1H, H4), $5.25(\mathrm{dd}, J=2.3,5.5 \mathrm{~Hz}, 1 \mathrm{H}, \mathrm{H} 1), 5.22-5.26(\mathrm{~m}, 1 \mathrm{H}, \mathrm{H} 3)$. 
The deoxyribopyranoside derivative (62\%) had: ${ }^{1} \mathrm{H}$ NMR $\left(\mathrm{CDCl}_{3}\right) \delta 2.09$ (s, 3H, Ac), 2.11 (s, 3H, Ac), 2.14-2.21 (m, 2H, H2, H2'), 3.43 (s, 3H, $\left.\mathrm{CH}_{3}\right), 4.27$ (d, $\left.J=4.1 \mathrm{~Hz}, 1 \mathrm{H}, \mathrm{H} 4\right)$, 5.05-5.08 (m, 1H, H3), $5.12(\mathrm{~d}, J=4.9 \mathrm{~Hz}, 1 \mathrm{H}, \mathrm{H} 1)$.

1,3,5-Di- $\boldsymbol{O}$-acetyl-2-deoxy-5,5'-dideuteroribose (123). Acetolysis of 132 (258 mg, $1.10 \mathrm{mmol}$ ) was carried out by dissolving it in $2 \mathrm{~mL}$ of acetic acid with $1 \mathrm{~mL}$ of acetic anhydride. The mixture was placed on a $0^{\circ} \mathrm{C}$ ice bath, and $\mathrm{H}_{2} \mathrm{SO}_{4}(40 \mu \mathrm{L}, 77.2 \mathrm{mg}, 0.75$ mmol) was added dropwise. The reaction was brought to room temperature and allowed to stir until it turned a deep purple color (roughly 15 mins). The residue was then stirred with crushed ice, followed by washing with $\mathrm{CH}_{2} \mathrm{Cl}_{2}$ two times. The organic layer was then washed with $\mathrm{NaHCO}_{3}$ twice and evaporated. The remaining oil was column chromatographed $(40 \% \rightarrow 80 \%$ EtOAc/Hexane) to give $236 \mathrm{mg}(82 \%)$ as a mixture of isomers. The deoxyribofuranose derivative $123(38 \%)$ had: ${ }^{1} \mathrm{H}$ NMR $\left(\mathrm{CDCl}_{3}\right) \delta 2.08(\mathrm{~s}$, 3H, Ac), 2.10 (s, 3H, Ac), 2.12 (s, 3H, Ac), 2.33-2.37 (m, 1H, H2), 2.49-2.54 (m, 1H, H2'), $4.32(\mathrm{~d}, J=3.5 \mathrm{~Hz}, 1 \mathrm{H}, \mathrm{H} 4), 5.38-5.41(\mathrm{~m}, 1 \mathrm{H}, \mathrm{H} 3) 6.43(\mathrm{dd}, J=2.4,5.7 \mathrm{~Hz}, 1 \mathrm{H}, \mathrm{H} 1)$.

The deoxyribopyranoside derivative (62\%) had: ${ }^{1} \mathrm{H}$ NMR $\left(\mathrm{CDCl}_{3}\right) \delta 2.10$ (s, 3H, Ac), 2.11 (s, 3H, Ac), 2.13 (s, 3H, Ac), 2.49-2.54 (m, 2H, H2, H2'), 4.44 (d, $J=3.0 \mathrm{~Hz}, 1 \mathrm{H}, \mathrm{H} 4)$, 5.14-5.18 (m, 1H, H3), $6.38(\mathrm{~d}, J=5.3 \mathrm{~Hz}, 1 \mathrm{H}, \mathrm{H} 1)$.

3',5'-Di-O-acetyl-2'-deoxy-5',5'-dideuterouridine (121). Protected sugar 123 (236 $\mathrm{mg}, 0.902 \mathrm{mmol}$ ) was placed in a flame-dried flask under $\mathrm{N}_{2}$ atmosphere and dissolved in $5 \mathrm{~mL} \mathrm{CH}{ }_{3} \mathrm{CN}$. Then, the crude TMS-protected uracil 122, synthesized in situ as previously described, ${ }^{105}$ was dissolved in $5 \mathrm{~mL} \mathrm{CH}_{3} \mathrm{CN}$ and added to the mixture. TMSOTf (30 $\mu \mathrm{L}$, $18.4 \mathrm{mg}, 0.083 \mathrm{mmol}$ ) was added and the reaction was stirred for $15 \mathrm{mins}$ at room temperature. Next, the mixture was placed in a $70^{\circ} \mathrm{C}$ oil bath to stir for $2 \mathrm{hrs}$ under $\mathrm{N}_{2}$. The 
solvent was then evaporated under vacuum, and the residue was dissolved in EtOAc followed by washing with $\mathrm{NaHCO}_{3}(\mathrm{X} 2)$ and brine. Column chromatography $(0 \% \rightarrow 5 \%$ $\left.\mathrm{MeOH} / \mathrm{CH}_{2} \mathrm{Cl}_{2}\right)$ gave $170 \mathrm{mg}(60 \%)$ of the mixture of isomers. The deoxyribofuranosyl derivative 121 (51\%) had: ${ }^{1} \mathrm{H}$ NMR $\left(\mathrm{CDCl}_{3}\right) \delta 2.08$ (s, 3H, Ac), 2.09 (s, 3H, Ac), 2.48-2.53 (m, 1H, H2'), 2.74-2.81 (m, 1H, H2"), 4.25 (d, J = 2.5 Hz, 1H, H4), 5.21-5.27 (m, 1H, H3'), $5.76(\mathrm{~d}, J=8.1 \mathrm{~Hz}, 1 \mathrm{H}, \mathrm{H} 5), 6.26\left(\mathrm{dd}, J=5.7,8.3 \mathrm{~Hz}, 1 \mathrm{H}, \mathrm{H} 1^{\prime}\right), 7.49(\mathrm{~d}, J=8.2 \mathrm{~Hz}, 1 \mathrm{H}$, H6).

The corresponding deoxyribopyranosyl derivative (49\%) had: ${ }^{1} \mathrm{H}$ NMR $\left(\mathrm{CDCl}_{3}\right) \delta 2.08(\mathrm{~s}$, 3H, Ac), 2.09 (s, 3H, Ac), 2.13-2.18 (m, 1H, H2', H2"), 4.57-4.59 (m, 1H, H4), 5.21-5.27 (m, 1H, H3'), 5.74 (d, $J=8.2 \mathrm{~Hz}, 1 \mathrm{H}, \mathrm{H} 5), 6.19\left(\mathrm{dd}, J=1.5,7.0 \mathrm{~Hz}, 1 \mathrm{H}, \mathrm{H} 1{ }^{\prime}\right), 7.47$ (d, $J=$ $8.2 \mathrm{~Hz}, 1 \mathrm{H}, \mathrm{H} 6)$.

\section{1-(3,5-Di- $O$-Acetyl-2-deoxy-5,5' -dideutero- $\beta$-D-ribofuranos-1-yl)-4-triazolyl-2-}

pyrimidinone (133). Protected uridine 121 (170 $\mathrm{mg}, 0.541 \mathrm{mmol})$ was dissolved in $3 \mathrm{~mL}$ of $\mathrm{CH}_{3} \mathrm{CN}$. In a separate flask, 1,2,4-1- $\mathrm{H}$-triazole was dissolved in $8 \mathrm{~mL} \mathrm{CH}_{3} \mathrm{CN}$ and placed at $0^{\circ} \mathrm{C}$. Then, $\mathrm{Et}_{3} \mathrm{~N}(646 \mu \mathrm{L}, 469 \mathrm{mg}, 4.64 \mathrm{mmol})$ and $\mathrm{POCl}_{3}(96 \mu \mathrm{L}, 157.3 \mathrm{mg}, 1.03 \mathrm{mmol})$ were added. Once the formation of triethylammonium chloride salt was observed, the solution of 121 was added and stirring was continued overnight. The reaction was quenched with $\mathrm{Et}_{3} \mathrm{~N}(451 \mu \mathrm{L})$ and $\mathrm{H}_{2} \mathrm{O}(248 \mu \mathrm{L})$ and stirred for $10 \mathrm{~min}$. Next, the solvent was evaporated, and the residue dissolved in EtOAc. The organic layer was washed with $\mathrm{NaHCO}_{3}, \mathrm{H}_{2} \mathrm{O}$, and brine. This mixture was column chromatographed $(50 \% \rightarrow 100 \%$ EtOAc/Hexane) to give $45 \mathrm{mg}(23 \%)$ of a mixture of isomers. The deoxyribofuranosyl derivative 133 (65\%) had: ${ }^{1} \mathrm{H}$ NMR $\left(\mathrm{CDCl}_{3}\right) \delta 2.11$ (s, 3H, Ac), 2.16 (s, 3H, Ac), 2.90-2.95 (m, 1H, H2'), 2.95-3.01 (m, 1H, H2"), 4.43 (d, J=2.6 Hz, 1H, H4), 5.24-5.29 (m, 2H, H3'), 
6.28-6.32 (m, 1H, H1'), 7.11 (d, $J=7.4 \mathrm{~Hz}, 1 \mathrm{H}, \mathrm{H} 5), 8.17$ (s, 1H, triazole), 8.31 (d, $J=7.3$ Hz, 1H, H6), 9.31 (s, 1H, triazole).

The deoxyribopyranosyl derivative (35\%) had: ${ }^{1} \mathrm{H}$ NMR $\left(\mathrm{CDCl}_{3}\right) \delta 2.14$ (s, 3H, Ac), 2.16 (s, 3H, Ac), 2.53-2.54 (m, 1H, H2'), 2.56-2.57 (m, 1H, H2"), 4.57 (d, 1H, J = 2.7 Hz, H4), 4.72-4.74 (m, 1H, H3'), 5.30 (d, $J=7.3 \mathrm{~Hz}, 1 \mathrm{H}, \mathrm{H} 5), 6.28-6.32\left(\mathrm{~m}, 1 \mathrm{H}, \mathrm{H} 1^{\prime}\right), 7.13$ (d, $J=$ $7.3 \mathrm{~Hz}, 1 \mathrm{H}, \mathrm{H6}), 8.15$ (s, $1 \mathrm{H}$, triazole), 9.29 (s, $1 \mathrm{H}$, triazole).

\section{1-(3,5-Di-O-acetyl-2-deoxy-5,5'-dideutero-ß-D-ribofuranos-1-yl)-4-azido-2-}

pyrimidinone (134). The 4-triazolyl 133 (45 mg, $0.123 \mathrm{mmol})$ was dissolved in $5 \mathrm{~mL}$ DMF, and heated at $50^{\circ} \mathrm{C}$. Acetic acid $(89 \mu \mathrm{L}, 93.7 \mathrm{mg}, 1.56 \mathrm{mmol})$ was added to the mixture. Then, $\mathrm{NaN}_{3}\left(50 \mathrm{mg}, 0.769 \mathrm{mmol}\right.$ ) was dissolved in $1 \mathrm{~mL}$ of $\mathrm{H}_{2} \mathrm{O}$ and added to the reaction. The resulting mixture was stirred at $50^{\circ} \mathrm{C}$ for 3 days. Once cooled, the mixture was partitioned between EtOAc and $\mathrm{H}_{2} \mathrm{O}$, followed by washing of the organic layer with $\mathrm{NaHCO}_{3}$ and brine. The resulting residue was column chromatographed $(50 \% \rightarrow 90 \%$ EtOAc/Hex) to give $18 \mathrm{mg}(45 \%)$ as a mixture of isomers. The deoxyribofuranosyl derivative 134 (68\%) had: ${ }^{1} \mathrm{H}$ NMR $\left(\mathrm{CDCl}_{3}\right) \delta 2.14$ (s, 3H, Ac), 2.17 (s, 3H, Ac), 2.78 (ddd, $J=2.0,5.7,14.4 \mathrm{~Hz}, 1 \mathrm{H}, \mathrm{H} 2$ '), 2.95-3.01 (m, 1H, H2"), 4.42 (d, $J=2.5 \mathrm{~Hz}, 1 \mathrm{H}, \mathrm{H} 4$ ), 5.28-5.31 (m, 1H, H3'), $6.52\left(\mathrm{dd}, J=5.7,8.1 \mathrm{~Hz}, 1 \mathrm{H}, \mathrm{H1} 1^{\prime}\right), 6.98(\mathrm{~d}, J=7.9 \mathrm{~Hz}, 1 \mathrm{H}, \mathrm{H} 5)$, $7.79(\mathrm{~d}, J=7.9 \mathrm{~Hz}, 1 \mathrm{H}, \mathrm{H} 6) ;{ }^{13} \mathrm{C} \mathrm{NMR}\left(\mathrm{CDCl}_{3}\right) \delta 20.9\left(\mathrm{CH}_{3} \mathrm{x} 2\right), 38.7\left(\mathrm{C}^{\prime}\right), 63.5\left(\mathrm{C} 5^{\prime} ;\right.$ low intensity pentet due to splitting to two deuterons), 73.9 (C4'), 83.4 (C3'), 87.1 (C1'), 93.2 (C5), 133.6 (C6), 142.5 (C4), 150.7 (C2), 169.8 (CO), 170.2 (CO); HRMS m/z calc for $\mathrm{C}_{13} \mathrm{H}_{13} \mathrm{D}_{2} \mathrm{~N}_{5} \mathrm{O}_{6}[\mathrm{M}+\mathrm{Na}]^{+}$362.1046, found 362.1030 .

The deoxyribopyranosyl derivative (32\%) had: ${ }^{1} \mathrm{H}$ NMR $\left(\mathrm{CDCl}_{3}\right) \delta 2.15$ (s, 3H, Ac), 2.19 (s, 3H, Ac), 2.26-2.32 (m, 1H, H2', H2"), 4.74-4.76 (m, 1H, H4), 5.33-5.35 (m, 1H, H3'), 
$6.47\left(\mathrm{dd}, J=1.5,7.0 \mathrm{~Hz}, 1 \mathrm{H}, \mathrm{H} 1^{\prime}\right), 6.94(\mathrm{~d}, J=7.9 \mathrm{~Hz}, 1 \mathrm{H}, \mathrm{H} 5), 7.71(\mathrm{~d}, J=7.9 \mathrm{~Hz}, 1 \mathrm{H}$, H6)

\section{CONCLUSION}

Two classes of gemcitabine analogues with lipophilic 4- $N$-alkyl chains have been synthesized. One class, bearing $\beta$-keto sulfonate moiety which has been utilized for subsequent ${ }^{18}$ F-fluorination (e.g., 56). The second, bearing a SCN-Bn-NOTA chelator has been employed for ${ }^{68} \mathrm{Ga}$ complexation (e.g., 65). These analogues were designed and synthesized to study their stability during ${ }^{18} \mathrm{~F}$ - or ${ }^{68} \mathrm{Ga}$-radiolabeling to potentially serve as new PET imaging radiotracers for gemcitabine.

The synthesis of 4- $N$-alkylgemcitabine bearing a $\beta$-keto tosylate moiety was undertaken to overcome the in vivo defluorination experienced by similar $4-N$-alkyl 
gemcitabine analogues due to the hydrogens present on carbon- $\beta$ to fluorine. Synthesis of this analogue began with the displacement of $p$-toulenesulfonylamino group in $4-\mathrm{N}$ tosylgemcitabine with 1-amino-10-undecene to afford 4- $\mathrm{N}$-alkylgemcitabine with a terminal olefin. Elaboration of the terminal alkene by dihydroxylation, regioselective tosylation, and oxidation gave the desired $\beta$-keto tosylate analogue (e.g., 56). Displacement of the tosylate group with fluorine was achieved using non-radiolabeling protocols to give 4- $N$-alkylgemcitabine analogue bearing a terminal $\alpha$-fluoromethyl ketone (e.g., 64). However, treatment of the protected $\beta$-keto tosylate, using standard ${ }^{18} \mathrm{~F}$-radiolabeling protocols, followed by immediate deprotection with gave $\alpha$-fluoromethyl ketone in low yields.

Synthesis of 4- $N$-alkylgemcitabine conjugated to SCN-Bn-NOTA was achieved by replacement of tosylamide in 4- $\mathrm{N}$-tosylgemcitabine with $\mathrm{N}$-Boc-1,3-propanediamine, followed deprotection to acquire 4- $N$-alkylgemcitabine with a reactive terminal amine (e.g. 67). Subsequent condensation with SCN-Bn-NOTA chelator gave novel NOTA-4- $N$ alkylgemcitabine analogue (e.g., 65). Ligand 65 was efficiently complexed with gallium(III) chloride $\left(\mathrm{GaCl}_{3}\right)$ giving the Gallium NOTA-4- $N$-alkylgemcitabine complex 68 in quantitative yield after 15 mins, as characterized by HRMS. Radiolabeling of $\mathbf{6 5}$ with ${ }^{68} \mathrm{Ga}$ gave $\left[{ }^{68} \mathrm{Ga}\right]-\mathbf{6 8}$ quantitatively after 5 mins. This radiotracer was injected onto BALC/c mice where it showed only minimal hepatobiliary excretion, and rapid renal clearance most likely due to the increased hydrophilicity due to the addition of the NOTA chelator.

Despite the fact that synthesis of the model adenosine 5'-diphosphate prodrug (e.g., 87) was successful, coupling of clofarabine 5'-monophosphate $\mathbf{8 1}$ with bis(benzoyloxy benzyl) phosphoramidite reagent $\mathbf{8 4}$ under several reaction conditions failed to produce desired 
diphosphate prodrug $\mathbf{8 8}$. Improvement of the purity of phosphoramidite, and exchange of triethylammonium acetate to simply triethylammonium counter ions in clofarabine 5'monophosphate may help with the synthesis of desired $\mathbf{8 8}$. Since no effective protocols for synthesis of $\mathbf{8 8}$ has been developed, our goals of synthesis of $\mathrm{C}^{2}{ }^{18} \mathrm{~F}$-clofarabine diphosphate prodrugs as potential new PET imaging radiotracers has not been further explored.

In my dissertation, I have reported the synthesis of several azido modified purine and pyrimidine analogues for use in EPR studies to characterize the guanyl, adenyl, and cytidyl aminyl radicals. The first class of azido analogues synthesized were the 2-azidopurine nucleosides including: 2-azido-2'-deoxyinosine 89, 2-azidoinsosine 91, 2-azido-2'deoxyadenosine 90, and 2-azidoadenosine 92 that were prepared for EPR elucidation of the guanyl-type 2-aminyl radical. The second class includes protected 6-azido-2'deoxyadenosine $\mathbf{1 0 7}$, which was synthesized to characterize the adenyl 6-aminyl radical that has been shown to be the source of certain lesions in DNA. ${ }^{124}$ The third class of compound were the azido-modified pyrimidine analogues that includes: 4-azido-3',5'-di$O$-acetyl-2'-deoxyuridine 112, 4-tetrazolo-2'-deoxyuridine 113, 4-azido-2',3',5'-tri- $O$ acetyluridine 114, and 4-tetrazolouridine 115, synthesized for the EPR investigation of the cytidyl 4-aminyl radical.

The ESR studies of electron addition to 2-azidoinosine analogues $\mathbf{8 9}$ and $\mathbf{9 0}$, or 4-azido 112 and tetrazole 113 clearly establish that position of the azido group in the base moiety dictates its reactivity towards electron addition. If the azido group is at the ortho position to ring nitrogens (N1 or N3) as in $\mathbf{8 9}$ and $\mathbf{9 0}$, or in ortho/para positions as in 6-azido-2'deoxyadenosine 107 , 4-azido 112 , or tetrazole 113 , these compounds produce $\mathrm{RN}_{3}{ }^{\circ}$ which 
is stable and does not rapidly convert to the aminyl radical, except in their excited state. Hence, these compounds did not show much radiasensitization in comparison to the significant radiosensitization observed from 5 -AmdU, ${ }^{106}$ (in which "isolated" from heterocyclic nucleobase azido group is readily converted to reactive aminyl radical) in our in vivo biological studies of these compounds in MDA-MB-231, MCF7 and U87 cell lines.

\section{REFERENCES}

(1) Parker, W. B. Chem. Rev. 2009, 109, 2880.

(2) Galmarini, C. M.; Mackey, J. R.; Dumontet, C. Leukemia 2001, 15, 875.

(3) Hertel, L. W.; Kroin, J. S.; Misner, J. W.; Tustin, J. M. J. Org. Chem. 1988, 53, 2406.

(4) Gesto, D. S.; Cerqueira, N. M. F. S. A.; Fernandes, P. A.; Ramos, M. J. Curr. Med. Chem. 2012, 19.

(5) Toschi, L. F., G.; Bartolini, S.; Gioia, V.; Cappuzzo, F. Future Oncol. 2005, 1, 7.

(6) Mini, E.; Nobili, S.; Caciagli, B.; Landini, I.; Mazzei, T. Ann. Oncol. 2006, 17 Suppl 5, v7. 
(7) Heinemann, V.; Hertel, L. W.; Grindey, G. B.; Plunkett, W. Cancer Res. 1998, 48, 4024.

(8) Stubbe, J.; Van Der Donk, W. A. Chem. Rev. 1998, 98, 705.

(9) Artin, E.; Wang, J.; Lohman, G. J.; Yokoyama, K.; Yu, G.; Griffin, R. G.; Bar, G.; Stubbe, J. Biochemistry 2009, 48, 11622.

(10) Gandhi, V.; Huang, P.; Xu, Y. Z.; Heinemann, V.; Plunkett, W. Adv. Exp. Med. Biol. 1991, 309A, 125.

(11) Aye, Y.; Brignole, E. J.; Long, M. J.; Chittuluru, J.; Drennan, C. L.; Asturias, F. J.; Stubbe, J. Chem. Biol. 2012, 19, 799.

(12) Veltkamp, S. A.; Pluim, D.; van Eijndhoven, M. A.; Bolijn, M. J.; Ong, F. H.; Govindarajan, R.; Unadkat, J. D.; Beijnen, J. H.; Schellens, J. H. Mol. Cancer Ther. 2008, $7,2415$.

(13) Hodge, L. S.; Taub, M. E.; Tracy, T. S. Drug Metab. Dispos. 2011, 39, 2013.

(14) Montgomery, J. A.; Shortnacy-Fowler, A. T.; Clayton, S. D.; Riordan, J. M.; Secrist, J. A. J. Med. Chem. 1992, 35, 397.

(15) Bonate, P. L.; Arthaud, L.; Cantrell, W. R., Jr.; Stephenson, K.; Secrist, J. A., 3rd; Weitman, S. Nat. Rev. Drug Discov. 2006, 5, 855.

(16) King, K. M.; Damaraju, V. L.; Vickers, M. F.; Yao, S. Y.; Lang, T.; Tackaberry, T. E.; Mowles, D. A.; Ng, A. M.; Young, J. D.; Cass, C. E. Mol. Pharmacol. 2006, 69, 346.

(17) Zhang, Y.; Secrist, J. A., III; Ealick, S. E. Acta Crystallogr. D Biol. Crystallogr. 2006, 62, 133.

(18) Parker, W. B.; Shaddix, S. C.; Chang, C.-H.; White, E. L.; Rose, L. M.; Brockman, R. W.; Shortnacy, A. T.; Montgomery, J. A.; Secrist, J. A.; Bennett, L. L. Cancer Res. 1991, 51, 2386.

(19) Parker, W. B.; Shaddix, S. C.; Rose, L. M.; Shewach, D. S.; Hertel, L. W.; Secrist, J. A.; Montgomery, J. A.; Bennett, L. L. Mol. Pharmacol. 1999, 55, 515.

(20) Xie, C.; Plunkett, W. Cancer Res. 1995, 55, 2847.

(21) Xie, K. C.; Plunkett, W. Cancer Res. 1996, 56, 3030. 
(22) Sylwestrowicz, T. A.; Ma, D. D. F.; Murphy, P. P.; Massaia, M.; Prentice, H. G.; Hoffbrand, A. V.; Greaves, M. F. Leukemia Res. 1982, 6, 475.

(23) Zimmermann, H. Biochem J. 1992, 285, 345.

(24) Rautio, J.; Kumpulainen, H.; Heimbach, T.; Oliyai, R.; Oh, D.; Järvinen, T.; Savolainen, J. Nat. Rev. Drug Discov. 2008, 7, 255.

(25) Jornada, D. H.; dos Santos Fernandes, G. F.; Chiba, D. E.; de Melo, T. R.; dos Santos, J. L.; Chung, M. C. Molecules 2015, 21, 42.

(26) Alexander, P.; Kucera, G.; Pardee, T. S. Crit. Rev. Oncol. Hematol. 2016, 100, 46.

(27) Li, F.; Maag, H.; Alfredson, T. J. Pharm. Sci. 2008, 97, 1109.

(28) Immordino, M. L.; Brusa, P.; Rocco, F.; Arpicco, S.; Ceruti, M.; Cattel, L. J. Control. Release 2004, 100, 331.

(29) Couvreur, P.; Stella, B.; Reddy, L. H.; Hillaireau, H.; Dubernet, C.; Desmaële, D.; Lepêtre-Mouelhi, S.; Rocco, F.; Dereuddre-Bosquet, N.; Clayette, P.; Rosilio, V.; Marsaud, V.; Renoir, J.-M.; Cattel, L. Nano Lett. 2006, 6, 2544.

(30) Bender, D. M.; Bao, J.; Dantzig, A. H.; Diseroad, W. D.; Law, K. L.; Magnus, N. A.; Peterson, J. A.; Perkins, E. J.; Pu, Y. J.; Reutzel-Edens, S. M.; Remick, D. M.; Starling, J. J.; Stephenson, G. A.; Vaid, R. K.; Zhang, D.; McCarthy, J. R. J. Med. Chem. 2009, 52, 6958.

(31) Bergman, A. M.; Adema, A. D.; Balzarini, J.; Bruheim, S.; Fichtner, I.; Noordhuis, P.; Fodstad, O.; Myhren, F.; Sandvold, M. L.; Hendriks, H. R.; Peters, G. J. Invest. New Drugs 2011, 29, 456.

(32) Dasari, M.; Acharya, A. P.; Kim, D.; Lee, S.; Lee, S.; Rhea, J.; Molinaro, R.; Murthy, N. Bioconjug. Chem. 2013, 24, 4.

(33) Pulido, J.; Sobczak, A. J.; Balzarini, J.; Wnuk, S. F. J. Med. Chem. 2014, 57, 191.

(34) Wickremsinhe, E.; Bao, J.; Smith, R.; Burton, R.; Dow, S.; Perkins, E. Pharmaceutics 2013, 5, 261.

(35) Rejiba, S.; Reddy, L. H.; Bigand, C.; Parmentier, C.; Couvreur, P.; Hajri, A. Nanomedicine 2011, 7, 841. 
(36) Maiti, S.; Park, N.; Han, J. H.; Jeon, H. M.; Lee, J. H.; Bhuniya, S.; Kang, C.; Kim, J. S. J. Am. Chem. Soc. 2013, 135, 4567.

(37) Yang, Z.; Lee, J. H.; Jeon, H. M.; Han, J. H.; Park, N.; He, Y.; Lee, H.; Hong, K. S.; Kang, C.; Kim, J. S. J. Am. Chem. Soc. 2013, 135, 11657.

(38) Liu, L.-H.; Qiu, W.-X.; Li, B.; Zhang, C.; Sun, L.-F.; Wan, S.-S.; Rong, L.; Zhang, X.-Z. Adv. Funct. Mater. 2016, 26, 6257.

(39) Pradere, U.; Garnier-Amblard, E. C.; Coats, S. J.; Amblard, F.; Schinazi, R. F. Chem. Rev. 2014, 114, 9154.

(40) Sofia, M. J.; Bao, D.; Chang, W.; Du, J.; Nagarathnam, D.; Rachakonda, S.; Reddy, P. G.; Ross, B. S.; Wang, P.; Zhang, H.-R.; Bansal, S.; Espiritu, C.; Keilman, M.; Lam, A. M.; Steuer, H. M. M.; Niu, C.; Otto, M. J.; Furman, P. A. J. Med. Chem. 2010, 53, 7202.

(41) McGuigan, C.; Cahard, D.; Sheeka, H. M.; De Clercq, E.; Balzarini, J. J. Med. Chem. 1996, 39, 1748.

(42) McGuigan, C.; Murziani, P.; Slusarczyk, M.; Gonczy, B.; Vande Voorde, J.; Liekens, S.; Balzarini, J. J. Med. Chem. 2011, 54, 7247.

(43) McGuigan, C.; Pathirana, R. N.; Balzarini, J.; De Clercq, E. J. Med. Chem. 1993, $36,1048$.

(44) McGuigan, C.; Derudas, M.; Bugert, J. J.; Andrei, G.; Snoeck, R.; Balzarini, J. Bioorg. Med. Chem. Lett. 2008, 18, 4364.

(45) Pertusati, F.; McGuigan, C. ChemComm 2015, 51, 8070.

(46) Meier, C.; Jessen, H. J.; Balzarini, J. Nucleic Acids Symp. Ser. (Oxf) 2008, 52, 83.

(47) Jessen, J. H.; Schulz, T.; Balzarini, J.; Meier, C. Angew. Chem. Int. Ed. 2008, 47, 8719

(48) Slusarczyk, M.; Lopez, M. H.; Balzarini, J.; Mason, M.; Jiang, W. G.; Blagden, S.; Thompson, E.; Ghazaly, E.; McGuigan, C. J. Med. Chem. 2014, 57, 1531.

(49) Derudas, M.; Carta, D.; Brancale, A.; Vanpouille, C.; Lisco, A.; Margolis, L.; Balzarini, J.; McGuigan, C. J. Med. Chem. 2009, 52, 5520.

(50) Schulz, T.; Balzarini, J.; Meier, C. ChemMedChem 2014, 9, 762. 
(51) Weinschenk, L.; Schols, D.; Balzarini, J.; Meier, C. J. Med. Chem. 2015, 58, 6114.

(52) Weinschenk, L.; Gollnest, T.; Schols, D.; Balzarini, J.; Meier, C. ChemMedChem 2015, 10, 891 .

(53) Pertenbreiter, F.; Balzarini, J.; Meier, C. ChemMedChem 2015, 10, 94.

(54) Daniels, S.; Tohid, S. F. M.; Velanguparackel, W.; Westwell, A. D. Expert Opin. Drug Discov. 2010, 5, 291.

(55) Cole, E. L.; Stewart, M. N.; Littich, R.; Hoareau, R.; Scott, P. J. H. Curr. Top. Med. Chem. 2014, 14, 875.

(56) Townsend, D. W. Ann. Acad. Med. Singapore 2004, 33, 133.

(57) Van Den Hoff, J. Amino Acids 2005, 29, 341.

(58) Van Der Veldt, A. A.; Smit, E. F.; Lammertsma, A. A. Front Oncol. 2013, 3, 208.

(59) Schlyer, D. J. Ann. Acad. Med. Singapore 2004, 33, 146.

(60) Alauddin, M. M. Am. J. Nucl. Med. Mol. Imaging 2011, $2,55$.

(61) Alauddin, M. M. J. Labelled Comp. Radiopharm. 2013, 56, 237.

(62) Radu, C. G.; Shu, C. J.; Nair-Gill, E.; Shelly, S. M.; Barrio, J. R.; Satyamurthy, N.; Phelps, M. E.; Witte, O. N. Nat. Med. 2008, 14, 783.

(63) Laing, R. E.; Walter, M. A.; Campbell, D. O.; Herschman, H. R.; Satyamurthy, N.; Phelps, M. E.; Czernin, J.; Witte, O. N.; Radu, C. G. Proc. Natl. Acad. Sci. U.S.A. 2009, $106,2847$.

(64) Lee, J. T.; Campbell, D. O.; Satyamurthy, N.; Czernin, J.; Radu, C. G. J. Nucl. Med. 2012, 53, 275.

(65) Kim, W.; Le, T. M.; Wei, L.; Poddar, S.; Bazzy, J.; Wang, X.; Uong, N. T.; Abt, E. R.; Capri, J. R.; Austin, W. R.; Van Valkenburgh, J. S.; Steele, D.; Gipson, R. M.; Slavik, R.; Cabebe, A. E.; Taechariyakul, T.; Yaghoubi, S. S.; Lee, J. T.; Sadeghi, S.; Lavie, A.; Faull, K. F.; Witte, O. N.; Donahue, T. R.; Phelps, M. E.; Herschman, H. R.; Herrmann, K.; Czernin, J.; Radu, C. G. Proc. Natl. Acad. Sci. U.S.A. 2016, 113, 4027. 
(66) Brown, K.; Dixey, M.; Weymouth-Wilson, A.; Linclau, B. Carbohydr. Res. 2014, $387,59$.

(67) Meyer, J. P., In Synthetic Routes to 18F-Labelled Gemcitabine and related 2'Fluoronucleosides. PhD Dissertation, Cardiff University, Wales, UK, 2014.

(68) Zipse, H.; Artin, E.; Wnuk, S.; Lohman, G. J. S.; Martino, D.; Griffin, R. G.; Kacprzak, S.; Kaupp, M.; Hoffman, B.; Bennati, M.; Stubbe, J.; Lees, N. J. Am. Chem. Soc. 2009, 131, 200.

(69) Adhikary, A.; Kumar, A.; Rayala, R.; Hindi, R. M.; Adhikary, A.; Wnuk, S. F.; Sevilla, M. D. J. Am. Chem. Soc. 2014, 136, 15646.

(70) Guo, X.; Leonard, P.; Ingale, S. A.; Seela, F. Chem. Eur. J. 2017, 23, 17740.

(71) Frost, A. B.; Brambilla, M.; Exner, R. M.; Tredwell, M. Angew. Chem. Int. Ed. Engl. 2019, 58, 472.

(72) Velikyan, I.; Maecke, H.; Langstrom, B. Bioconjugate Chem. 2008, 19, 569.

(73) Brechbiel, M. W. Q. J. Nucl. Med. Mol. Imaging 2008, 52, 166.

(74) Notni, J.; Pohle, K.; Wester, H.-J. EJNMMI Res. 2012, 2, 28.

(75) Banerjee, S. R.; Pomper, M. G. Appl. Radiat. Isot. 2013, 76, 2.

(76) Färber, S. F.; Wurzer, A.; Reichart, F.; Beck, R.; Kessler, H.; Wester, H.-J.; Notni, J. ACS Omega 2018, 3, 2428.

(77) Jeong, J. M.; Hong, M. K.; Chang, Y. S.; Lee, Y. S.; Kim, Y. J.; Cheon, G. J.; Lee, D. S.; Chung, J. K.; Lee, M. C. J. Nucl. Med. 2008, 49, 830.

(78) Hedley, D. W.; Tripp, E. H.; Slowiaczek, P.; Mann, G. J. Cancer Res. 1988, 48, 3014.

(79) Chitambar, C. R.; Narasimhan, J.; Guy, J.; Sem, D. S.; O'Brien, W. J. Cancer Res. 1991, 51, 6199.

(80) Haq, R. U.; Wereley, J. P.; Chitambar, C. R. Exp. Hematol. 1995, 23, 428.

(81) Andre, J. P.; Maecke, H. R.; Zehnder, M.; Macko, L.; Akyel, K. G. Chem. Commun. 1998, 1301. 
(82) Eisenwiener, K.-P.; Prata, M. I. M.; Buschmann, I.; Zhang, H.-W.; Santos, A. C.; Wenger, S.; Reubi, J. C.; Mäcke, H. R. Bioconjugate Chem. 2002, 13, 530.

(83) Hong, H.; Zhang, Y.; Nayak, T. R.; Engle, J. W.; Wong, H. C.; Liu, B.; Barnhart, T. E.; Cai, W. J. Nucl. Med. 2012, 53, 1748.

(84) Cooke, M. S.; Evans, M. D.; Dizdaroglu, M.; Lunec, J. F.A.S.E.B. J. 2003, 17, 1195.

(85) Von Sonntag, C. Free-Radical-Induced DNA Damage and Its Repair; Springer: Germany, 2006.

(86) Visconti, R.; Grieco, D. Curr. Opin. Drug Discov. Devel. 2009, 12, 240.

(87) Reuter, S.; Gupta, S. C.; Chaturvedi, M. M.; Aggarwal, B. B. Free Radic. Biol. Med. 2010, 49, 1603.

(88) Buxton, G. V.; Greenstock, C. L.; Helman, W. P.; Ross, A. B. J. Phys. Chem. Ref. Data 1988, 17, 513.

(89) Steenken, S. Chem. Rev. 1989, 89, 503.

(90) Adhikary, A.; Kumar, A.; Becker, D.; Sevilla, M. D. J. Phys. Chem. B 2006, 110, 24171.

(91) Naumov, S.; Von Sonntag, C. Radiat. Res. 2008, 169, 364.

(92) Adhikary, A.; Khanduri, D.; Sevilla, M. D. J. Am. Chem. Soc. 2009, 131, 8614.

(93) Adhikary, A.; Kumar, A.; Munafo, S. A.; Khanduri, D.; Sevilla, M. D. Phys. Chem. Chem. Phys. 2010, 12, 5353.

(94) Khanduri, D.; Adhikary, A.; Sevilla, M. D. J. Am. Chem. Soc. 2011, 133, 4527.

(95) Adhikary, A.; Kumar, A.; Becker, D.; Sevilla, M. D. In Encyclopedia of Radicals in Chemistry, Biology and Materials; Chatgilialoglu, C., Struder, A., Eds.; John Wiley \& Sons Ltd: Chichester, UK, 2012, p 1371.

(96) Gimisis, T.; Cismaş, C. Eur. J. Org. Chem. 2006, 2006, 1351.

(97) Naumov, S.; Von Sonntag, C. Radiat. Res. 2008, 169, 355.

(98) Kumar, A.; Pottiboyina, V.; Sevilla, M. D. J. Phys. Chem. B 2011, 115, 15129. 
(99) Rak, J.; Chomicz, L.; Wiczk, J.; Westphal, K.; Zdrowowicz, M.; Wityk, P.; Zyndul, M.; Makurat, S.; Golon, L. J. Phys. Chem. B 2015, 119, 8227.

(100) Joiner, M. C.; Van Der Kogel, A. J.; Steel, G. G. In Basic Clinical Radiobiology; Joiner, M. C., Van Der Kogel, A., Eds.; CRC Press: London, 2009.

(101) Choy, H.; MacRae, R. M. In Chemoradiation in cancer therapy; Choy, H., Ed.; Humana Press: Totowa, NJ, 2003, p 3.

(102) Oronsky, B. T.; Knox, S. J.; Scicinski, J. Transl. Oncol. 2011, 4, 189.

(103) Sonntag, C. v. Free-radical-induced DNA damage and its repair: a chemical perspective; Berlin ; New York : Springer, c2006., 2006.

(104) Adhikary, A.; Khanduri, D.; Pottiboyina, V.; Rice, C. T.; Sevilla, M. D. J. Phys. Chem. B 2010, 114, 9289.

(105) Mudgal, M.; Rishi, S.; Lumpuy, D. A.; Curran, K. A.; Verley, K. L.; Sobczak, A. J.; Dang, T. P.; Sulimoff, N.; Kumar, A.; Sevilla, M. D.; Wnuk, S. F.; Adhikary, A. J. Phys. Chem. B 2017, 121, 4968.

(106) Wen, Z.; Peng, J.; Tuttle, P. R.; Ren, Y.; Garcia, C.; Debnath, D.; Rishi, S.; Hanson, C.; Ward, S.; Kumar, A.; Liu, Y.; Zhao, W.; Glazer, P. M.; Liu, Y.; Sevilla, M. D.; Adhikary, A.; Wnuk, S. F. Org. Lett. 2018, 7400.

(107) Kaloudis, P.; D’Angelantonio, M.; Guerra, M.; Spadafora, M.; Cismas, C.; Gimisis, T.; Mulazzani, Q. G.; Chatgilialoglu, C. J. Am. Chem. Soc. 2009, 131, 15895.

(108) Di Mascio, P.; Martinez, G. R.; Miyamoto, S.; Ronsein, G. E.; Medeiros, M. H. G.; Cadet, J. Chem. Rev. 2019, 119, 2043.

(109) Shukla, L. I.; Adhikary, A.; Pazdro, R.; Becker, D.; Sevilla, M. D. Nucleic Acid Res. 2004, 32, 6565.

(110) Adhikary, A.; Kumar, A.; Heizer, A. N.; Palmer, B. J.; Pottiboyina, V.; Liang, Y.; Wnuk, S. F.; Sevilla, M. D. J. Am. Chem. Soc. 2013, 135, 3121.

(111) Adriaanse, C.; Sulpizi, M.; VandeVondele, J.; Sprik, M. J. Am. Chem. Soc. 2009, $131,6046$.

(112) Cadet, J.; Berger, M.; Buchko, G. W.; Joshi, P. C.; Raoul, S.; Ravanat, J.-L. J. Am. Chem. Soc. 1994, 116, 7403. 
(113) Pratviel, G.; Meunier, B. Chem. Eur. J. 2006, 12, 6018.

(114) Candeias, L. P.; Steenken, S. Chem. Eur. J. 2000, 6, 475.

(115) Chatgilialoglu, C.; D'Angelantonio, M.; Guerra, M.; Kaloudis, P.; Mulazzani, Q. G. Angew. Chem. Int. Ed. Engl. 2009, 48, 2214.

(116) Vieira, A. J. S. C.; Steenken, S. J. Am. Chem. Soc. 1990, 112, 6986.

(117) Breen, A. P.; Murphy, J. A. Free Radic. Biol. Med. 1995, 18, 1033.

(118) Chatgilialoglu, C.; Caminal, C.; Altieri, A.; Vougioukalakis, G. C.; Mulazzani, Q. G.; Gimisis, T.; Guerra, M. J. Am. Chem. Soc. 2006, 128, 13796.

(119) Kuttappan-Nair, V.; Samson-Thibault, F.; Wagner, J. R. Chem. Res. Toxicol. 2010, $23,48$.

(120) Zheng, L.; Lin, L.; Qu, K.; Adhikary, A.; Sevilla, M. D.; Greenberg, M. M. Org. Lett. 2017, 19, 6444.

(121) Steenken, S. J. Chem. Soc., Faraday Trans 1987, 83, 113.

(122) Wagner, J. R.; van Lier, J. E.; Berger, M.; Cadet, J. J. Am. Chem. Soc. 1995, 116, 2235.

(123) Hildenbrand, K.; Behrens, G.; Schulte-Frohlinde, D. J. Chem. Soc. Perkin Trans. II 1989, 283.

(124) Sun, H.; Zheng, L.; Greenberg, M. M. J. Am. Chem. Soc. 2018, 140, 11308.

(125) Adhikary, A.; Kumar, A.; Bishop, C. T.; Wiegand, T. J.; Hindi, R. M.; Adhikary, A.; Sevilla, M. D. J. Phys. Chem. B 2015, 119, 11496.

(126) Anderson, R. F.; Shinde, S. S.; Maroz, A. J. Am. Chem. Soc. 2006, 128, 15966.

(127) Johnson, J. A.; Thomas, H. J.; Schaeffer, H. J. J. Am. Chem. Soc. 1958, 80, 699.

(128) Kotra, L. P.; Manouilov, K. K.; Cretton-Scott, E.; Sommadossi, J. P.; Boudinot, F. D.; Schinazi, R. F.; Chu, C. K. J. Med. Chem. 1996, 39, 5202.

(129) Robins, M. J.; Mullah, K. B.; Wnuk, S. F.; Dalley, N. K. J. Org. Chem. 1992, 57, 2357. 
(130) Kotra, L. P.; Wang, P.; Bartlett, M. G.; Shanmuganathan, K.; Xu, Z.; Cavalcanti, S.; Newton, M. G.; Chu, C. K. J. Org. Chem. 1997, 62, 7267.

(131) Koudriakova, T.; Manouilov, K. K.; Shanmuganathan, K.; Kotra, L. P.; Boudinot, F. D.; Cretton-Scott, E.; Sommadossi, J.-P.; Schinazi, R. F.; Chu, C. K. J. Med. Chem. 1996, 39, 4676.

(132) Horwitz, J. P.; Chua, J.; Noel, M. J. Org. Chem. 1964, 29, 2076.

(133) Norbeck, D. W. In Annual Reports in Medicinal Chemistry; Bristol, J. A., Ed.; Academic Press: 1990; Vol. 25, p 149.

(134) Ozols, K.; Cīrule, D.; Novosjolova, I.; Stepanovs, D.; Liepinsh, E.; Bizdēna, Ē.; Turks, M. Tetrahedron Lett. 2016, 57, 1174.

(135) Zayas, J.; Annoual, M.; Das, J. K.; Felty, Q.; Gonzalez, W. G.; Miksovska, J.; Sharifai, N.; Chiba, A.; Wnuk, S. F. Bioconjugate Chem. 2015, 26, 1519.

(136) Cosyn, L.; Palaniappan, K. K.; Kim, S.-K.; Duong, H. T.; Gao, Z.-G.; Jacobson, K. A.; Van Calenbergh, S. J. Med. Chem. 2006, 49, 7373.

(137) Gupte, A.; Boshoff, H. I.; Wilson, D. J.; Neres, J.; Labello, N. P.; Somu, R. V.; Xing, C.; Barry, C. E.; Aldrich, C. C. J. Med. Chem. 2008, 51, 7495.

(138) Zhang, H. W.; Zhou, L.; Coats, S. J.; McBrayer, T. R.; Tharnish, P. M.; Bondada, L.; Detorio, M.; Amichai, S. A.; Johns, M. D.; Whitaker, T.; Schinazi, R. F. Bioorg. Med. Chem. Lett. 2011, 21, 6788.

(139) Lakshman, M. K.; Kumar, A.; Balachandran, R.; Day, B. W.; Andrei, G.; Snoeck, R.; Balzarini, J. J. Org. Chem. 2012, 77, 5870.

(140) Lakshman, M. K.; Singh, M. K.; Parrish, D.; Balachandran, R.; Day, B. W. J. Org. Chem. 2010, 75, 2461.

(141) Lioux, T.; Gosselin, G.; Mathé, C. Eur. J. Org. Chem. 2003, 2003, 3997.

(142) Wada, T.; Mochizuki, A.; Higashiya, S.; Tsuruoka, H.; Kawahara, S.-i.; Ishikawa, M.; Sekine, M. Tetrahedron Lett. 2001, 42, 9215.

(143) Fabrega, C.; Garcia, R. G.; Diaz, A. R.; Eritja, R. Biol. Chem. 1998, 379, 527.

(144) Sylvers, L. A.; Wower, J. Bioconjugate Chem. 1993, 4, 411. 
(145) Xu, Y.-Z.; Swann, P. F. Nucleosides and Nucleotides 1995, 14, 939.

(146) Higashiya, S.; Kaibara, C.; Fukuoka, K.; Suda, F.; Ishikawa, M.; Yoshida, M.; Hata, T. Bioorg. Med. Chem. Lett. 1996, 6, 39.

(147) Holmes, R. E.; Robins, R. K. J. Am. Chem. Soc. 1965, 87, 1772.

(148) Frieden, M.; Avino, A.; Eritja, R. Nucleosides Nucleotides Nucleic Acids 2003, 22, 193.

(149) Bae, S.; Lakshman, M. K. J. Am. Chem. Soc. 2009, 131, 17993.

(150) De Napoli, L.; Mayol, L.; Piccialli, G.; Rossi, M.; Santacroce, C. J. Heterocycl. Chem. 1986, 23, 1401.

(151) Kotra, L. P.; Wang, P.; Bartlett, M. G.; Shanmuganathan, K.; Xu, Z.; Cavalcanti, S.; Newton, M. G.; Chu, C. K. J. Org. Chem. 1997, 62, 7267.

(152) Gourdain, S.; Petermann, C.; Harakat, D.; Clivio, P. Nucleosides Nucleotides Nucleic Acids 2010, 29, 542.

(153) Shiau, G. T.; Schinazi, R. F.; Chen, M. S.; Prusoff, W. H. J. Med. Chem. 1980, 23, 127.

(154) Divakar, K. J.; Reese, C. B. J. Chem. Soc., Perkin Trans. 11982.

(155) Salic, A.; Mitchison, T. J. Proc. Natl. Acad. Sci. U.S.A 2008, 105, 2415.

(156) Evans, R. K. Biochemistry 1987, 26, 269.

(157) Sunthankar, P.; Pastuszak, I.; Rooke, A.; Elbein, A. D.; van de Rijn, I.; Canfield, W. M.; Drake, R. R. Anal. Biochem. 1998, 258, 195.

(158) Barawkar, D. A.; Ganesh, K. N. Bioorg. Med. Chem. Lett. 1993, 3, 347.

(159) Gourdain, S.; Martinez, A.; Petermann, C.; Harakat, D.; Clivio, P. J. Org. Chem. 2009, 74, 6885 .

(160) Krim, J.; Taourirte, M.; Grünewald, C.; Krstic, I.; Engels, J. Synthesis 2013, 45, 396.

(161) Neef, A. B.; Luedtke, N. W. ChemBioChem 2014, 15, 789. 
(162) Hein, C. D.; Liu, X. M.; Wang, D. Pharm. Res. 2008, 25, 2216.

(163) Weber, L. Drug Discov. Today Technol. 2004, 1, 261.

(164) Kolb, H. C.; Sharpless, K. B. Drug Discov. Today 2003, 8, 1128.

(165) Meghani, N. M.; Amin, H. H.; Lee, B. J. Drug Discov.Today 2017, 22, 1604.

(166) Lin, P. C.; Ueng, S. H.; Yu, S. C.; Jan, M. D.; Adak, A. K.; Yu, C. C.; Lin, C. C. Org. Lett. 2007, 9, 2131.

(167) White, M. A.; Johnson, J. A.; Koberstein, J. T.; Turro, N. J. J. Am. Chem. Soc. 2006, $128,11356$.

(168) Baskin, J. M.; Prescher, J. A.; Laughlin, S. T.; Agard, N. J.; Chang, P. V.; Miller, I. A.; Lo, A.; Codelli, J. A.; Bertozzi, C. R. Proc. Natl. Acad. Sci. U.S.A 2007, 104, 16793.

(169) Laughlin, S. T.; Bertozzi, C. R. ACS Chem. Biol. 2009, 4, 1068.

(170) Belardi, B.; de la Zerda, A.; Spiciarich, D. R.; Maund, S. L.; Peehl, D. M.; Bertozzi, C. R. Angew. Chem. Int. Ed. Engl. 2013, 52, 14045.

(171) Cīrule, D.; Ozols, K.; Platnieks, O.; Bizdēna, Ē.; Mālinaa, I.; Turks, M. Tetrahedron 2016, $72,4177$.

(172) Amblard, F.; Cho, J. H.; Schinazi, R. F. Chem. Rev. 2009, 109, 4207.

(173) Ikehara, M.; Uesugi, S.; Yoshida, K. Biochemistry 1972, 11, 830.

(174) Sarma, R. H.; Lee, C.-H.; Evans, F. E.; Yathindra, N.; Sundaralingam, M. J. Am. Chem. Soc. 1974, 96, 7337.

(175) Gramlich, P. M.; Wirges, C. T.; Manetto, A.; Carell, T. Angew. Chem. Int. Ed. Engl. 2008, 47,8350 .

(176) Amblard, F.; Cho, J. H.; Schinazi, R. F. Chem. Rev. 2009, 109, 4207.

(177) Lee, Y. S.; Park, S. M.; Kim, H. M.; Park, S. K.; Lee, K.; Lee, C. W.; Kim, B. H. Bioorg. Med. Chem. Lett. 2009, 19, 4688.

(178) Efthymiou, T.; Gong, W.; Desaulniers, J. P. Molecules 2012, 17, 12665. 
(179) Kore, A. R.; Charles, I. Curr. Org. Chem. 2013, 17, 2164.

(180) El-Sagheer, A. H.; Brown, T. Chem. Soc. Rev. 2010, 39, 1388.

(181) Kukwikila, M.; Gale, N.; El-Sagheer, A. H.; Brown3, T.; Tavassoli, A. Nat. Chem. 2017, 9, 1089.

(182) Kolb, H. C.; Finn, M. G.; Sharpless, K. B. Angew. Chem. Int. Ed. Engl. 2001, 40, 2004.

(183) Tera, M.; StellaM.K.Glasauer; Luedtke, N. W. ChemBioChem 2018, 19, 1939.

(184) Ren, X.; El-Sagheer, A. H.; Brown, T. Analyst 2015, 140, 2671.

(185) Katritzky, A. R.; Rees, C. W. Comprehensive heterocyclic chemistry: the structure, reactions, synthesis, and uses of heterocyclic compounds; Pergamon Press: Oxford, 1984.

(186) Himo, F.; Demko, Z. P.; Noodleman, L.; Sharpless, K. B. J. Am. Chem. Soc. 2002, 124,12210 .

(187) Butler, R. N. In Advances in Heterocyclic Chemistry Katritzky, A. R., Boulton, A. J., Eds.; Academic Press: 1977; Vol. 21, p 323.

(188) Neugebauer, F. A. Angew. Chem. Int. Ed. Engl. 1973, 12, 455.

(189) Neugebauer, F. A.; Russell, G. A. J. Org. Chem. 1968, 33, 2744.

(190) Neugebauer, F. A. Tetrahedron Lett. 1968, 17, 2129.

(191) Neugebauer, F. A. Tetrahedron 1970, 26, 4843.

(192) Carroll Temple, J.; W. C. Coburn, J.; Thorpe, M. C.; Montgomery, J. A. J. Org. Chem. 1965, 30, 2395.

(193) Temple, C.; McKee, R. L.; Montgomery, J. A. J. Org. Chem. 1965, 30, 829.

(194) Carroll Temple, J.; Thorpe, M. C.; W. C. Coburn, J.; Montgomery, J. A. J. Org. Chem. 1966, 31, 935.

(195) Temple, C.; Kussner, C. L.; Montgomery, J. A. J. Org. Chem. 1966, 31, 2210. 
(196) Thomann, A.; Zapp, J.; Hutter, M.; Empting, M.; Hartmann, R. W. Org. Biomol. Chem. 2015, 13, 10620.

(197) Könnecke, A.; Dörre, R.; Kleinpeter, E.; Lippmann, E. Tetrahedron 1979, 35, 1957.

(198) Peyrane, F.; Cesario, M.; Clivio, P. J. Org. Chem. 2006, 71, 1742.

(199) Peyrane, F.; Clivio, P. Photochem. Photobiol. Sci. 2013, 12, 1366.

(200) Fomich, M. A.; Kvach, M. V.; Navakouski, M. J.; Weise, C.; Baranovsky, A. V.; Korshun, V. A.; Shmanai, V. V. Org. Lett. 2014, 16, 4590.

(201) Saneyoshi, M. Chem. Pharm. Bull. 1968, 16, 1616.

(202) Shu, C. J.; Campbell, D. O.; Lee, J. T.; Tran, A. Q.; Wengrod, J. C.; Witte, O. N.; Phelps, M. E.; Satyamurthy, N.; Czernin, J.; Radu, C. G. J. Nucl. Med. 2010, 51, 1092.

(203) Moysan, E.; Bastiat, G.; Benoit, J. P. Mol. Pharm. 2013, 10, 430.

(204) Pulido, J., In Design and Synthesis of 4-N-Alkanoyl and 4-N-Alkyl Gemcitabine Analogues Suitable for Positron Emission Tomography. PhD Dissertation, Florida International University, Miami, FL, 2014.

(205) Gonzalez, C.; Sanchez, A.; Collins, J.; Lisova, K.; Lee, J. T.; Michael van Dam, R.; Alejandro Barbieri, M.; Ramachandran, C.; Wnuk, S. F. Eur. J. Med. Chem. 2018, 148, 314.

(206) Gonzalez, C.; de Cabrera, M.; Wnuk, S. F. Nucleosides Nucleotides Nucleic Acids 2018, 37, 248.

(207) Pulido, J.; de Cabrera, M.; Sobczak, A. J.; Amor-Coarasa, A.; McGoron, A. J.; Wnuk, S. F. Bioorg. Med. Chem. 2018, 26, 5624.

(208) Amor-Coarasa, A.; Milera, A.; Carvajal, D.; Gulec, S.; McGoron, A. J. Int. J. Mol. Imag. 2014, 2014, 269365.

(209) We would like to thank Dr. Radu at University of California Los Angeles for sharing with us unpublished results on clofarabine ProTides and their dephosphorylation by 5 '-nucleotidases in cell studies.

(210) Yoshikawa M, K. T., Takenishi T Tetrahedron Lett. 1967, 8, 5065. 
(211) Robins, M. J.; Uznanski, B. Can. J. Chem. 1981, 59, 2601.

(212) Francom, P. R., Morris J. J. Org. Chem. 2003, 68, 666.

(213) Janeba, Z. F., Paula; Robins, Morris J. J. Org. Chem. 2003, 68, 989.

(214) Pankiewicz, K. W.; Krzeminski, J.; Ciszewski, L. A.; Ren, W.-Y.; Watanabe, K. A. J. Org. Chem. 1992, 57, 553.

(215) Bauta, W. E.; Schulmeier, B. E.; Burke, B.; Puente, J. F.; William R. Cantrell, J.; Lovett, D.; Goebel, J.; Anderson, B.; Ionescu, D.; Guo, R. Org. Process Res. Dev. 2004, 8, 889.

(216) Bauta, W. E.; Schulmeier, B. E.; Burke, B.; Puente, J. F.; Cantrell, W. R.; Lovett, D.; Goebel, J.; Anderson, B.; Ionescu, D.; Guo, R. C. Org. Process Res. Dev. 2004, 8, 889.

(217) Kovacs, T.; Otvos, L. Tetrahedron Lett. 1988, 29, 4525.

(218) Cremosnik, G. S.; Hofer, A.; Jessen, H. J. Angew. Chem. Int. Ed. Engl. 2014, 53, 286.

(219) Adhikary, A.; Becker, D.; Sevilla, M. D. In Applications of EPR in Radiation Research; Lund, A., Shiotani, M., Eds.; Springer: Berlin, Heidelberg, 2014, p 299.

(220) Greenberg, M. M. Radiat. Phys. Chem. 2016, 128, 82.

(221) Frederiksen, S. Arch. Biochem. Biophys. 1966, 113, 383.

(222) Diaz, A. R.; Eritja, R.; Garcia, R. G. Nucleosides and Nucleotides 1997, 16, 2035.

(223) Himmelsbach, F.; Schulz, B. S.; Trichinger, T.; Charubala, R.; Pfleidere, W. Tetrahedron 1984, 40, 59.

(224) Adhikary, A.; Kumar, A.; Khanduri, D.; Sevilla, M. D. J. Am. Chem. Soc. 2008, $130,10282$.

(225) Robins, M. J.; Basom, G. L. Can. J. Chem. 1973, 51, 3161.

(226) We would like to thank Dr. Adhikary and Dr. Sevilla at Oakland University for the excellent collaboration and for sharing with us the unpublished results of EPR studies of radiation-produced electron addition to azido-modified nucleosides. 
(227) Adhikary, A.; Malkhasian, A. Y.; Collins, S.; Koppen, J.; Becker, D.; Sevilla, M. D. Nucleic Acids Res. 2005, 33, 5553.

(228) Close, D. M. Radiat. Res. 1997, 147, 663.

(229) Adhikary, A.; Kumar, A.; Palmer, B. J.; Todd, A. D.; Heizer, A. N.; Sevilla, M. D. Int. J. Radiat. Biol. 2014, 90, 433.

(230) Adhikary, A.; Becker, D.; Collins, S.; Koppen, J.; Sevilla, M. D. Nucleic Acid Res. 2006, 34, 1501 .

(231) Boussicault, F.; Kaloudis, P.; Caminal, C.; Mulazzani, Q. G.; Chatgilialoglu, C. J. Am. Chem. Soc. 2008, 130, 8377.

(232) Niedballa, U.; Vorbruggen, H. J. Org. Chem. 1974, 39, 3654.

(233) Vorbruggen, H.; Krolikiewicz, K.; Bennua, B. Chem. Ber. 1981, 114, 1234.

(234) Baud, M. V.; Chavis, C.; Lucas, M.; Imbach, J. L. Tetrahedron Lett. 1990, 31, 4437.

(235) Gold, A.; Sangaiah, R. Nucleosides and Nucleotides 1990, 9, 907.

(236) This is an Accepted Manuscript of an article published by Taylor \& Francis in Nucleosides, Nucleotides and Nucleic Acids, on 11 May 2018, available online at https://doi.org/10.1080/15257770.2018.1465186.

(237) Janeba, Z.; Francom, P.; Robins, M. J. J. Org. Chem. 2003, 68, 989.

(238) Wenska, G.; Skalski, B.; Gdaniec, Z. Can. J. Chem. 1992, 70, 856. 
VITA

\section{MARIA E. DE CABRERA}

Born Cordoba, Argentina

2011-2015

2015-2018

2015-2019

2019
B.S. Chemistry

Florida International University

Miami, FL

M.S. Chemistry

Florida International University

Miami, FL

NRC Graduate Fellowship

Florida International University

Miami, FL

SoFLACS Graduate Travel Award 
2018-2019

Doctoral Candidate

Florida International University

Miami, FL

\section{PUBLICATIONS AND PRESENTATIONS}

- Maria de Cabrera, Cesar Gonzalez, Natasha Sulimoff, Stanislaw F. Wnuk. "Synthesis of 4-N-Alkyl Gemcitabine Analog Bearing $\beta$-Keto Tosylate Moiety Suitable for 18FLabeling" 253rd ACS National Meeting- San Francisco, CA (April 2nd to April 5th, 2017) [Poster Presentation]

- Cesar Gonzalez, Maria de Cabrera, Stanislaw F. Wnuk. "Gemcitabine analogues with 4- $\mathrm{N}$-alkyl chain modified with fluoromethyl ketone group." Nucleosides Nucleotides and Nucleic Acids, 2018, 37, 248-260.

- Maria de Cabrera, Stanislaw F. Wnuk. "Synthesis of Diphosphate ProTides for Enhancement of Cellular Metabolism" 255th ACS National Meeting- New Orleans, LA (March 18th to March 22nd, 2018) [Poster Presentation]

- Jesse Pulido, Maria de Cabrera, Adam J. Sobczak, Alejandro Amor-Coarasa, Anthony J. McGoron, Stanislaw F. Wnuk. "4- $N$-Alkanoyl and 4- $N$-Alkyl Gemcitabine Analogues with NOTA Chelators for 68-Gallium Labelling." Bioorganic Med. Chem., 2018, 26, 5624-5630

- Maria de Cabrera, Jesse Pulido, Adam J. Sobczak, Alejandro Amor-Coarasa, Anthony J. McGoron, and Stanislaw F. Wnuk. "4-N-Alkyl Gemcitabine Analogues with NOTA Chelators for 68-Gallium Labelling" $257^{\text {th }}$ ACS National Meeting- Orlando, FL (March $31^{\text {st }}$ to April $4^{\text {th }}$ ) [Oral Presentation]

- Yong Liang, Zhiwei Wen, Maria de Cabrera, Hasan Howlader, Stanislaw F. Wnuk. Purines. Science of Synthesis, 2nd ed., Vol. 16; Thieme Chemistry. (In progress)

- Maria de Cabrera, Alexandru Barbolovici, Samuel Ward, Ratan Sadhukhan, Rupak Pathak, Anil Kumar, Michael D. Sevilla, Amitava Adhikary, and Stanislaw F. Wnuk. "Site of azido substitution in the nucleobase dictates electron-mediated aminyl radical formation and its subsequent reactions." (Manuscript in preparation) 
